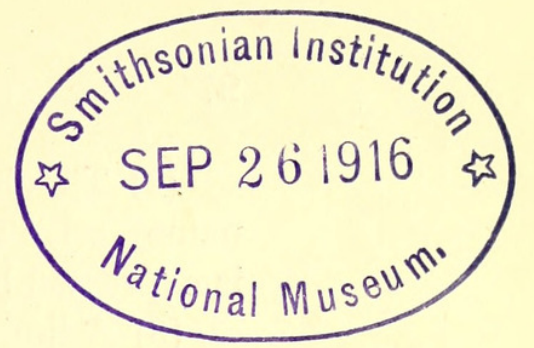

\title{
A RECORD OF PLANTS COLLECTED IN SOUTHERN RHODESIA.
}

\author{
Arranged on Engler's System.
}

Compiled by Fred. Eyles, F.L.S., 1915.

\section{INTRODUCTION.}

When first I began to collect the plants of Southern Rhodesia, I found it difficult, with the small library at my disposal, to trace descriptions of my specimens or to discover the records of previous collectors. For my own information, I then started a card-index in which I registered all available records of plants taken in this country, and added, wherever possible, references to the Floras and other standard publications wherein the various Rhodesian plants were described.

From this beginning the following work has sprung, and I publish it now because I feel the RECORD, imperfect though it is, should be made accessible to students of African botany. I also hope it may be of assistance to workers in the Rhodesian field and encourage others to collect and study the plants of this country.

This ReCord includes representatives of 160 Families, 869 Genera, and 2397 Species, besides 112 Varieties.

The "flowering" plants are arranged on Dr. Engler's system as set out in the Genera Siphonogamarum of Dr. C. G. De Dalla Torre \& Dr. H. Harms, 1900-1907.

The ferns and fern-allies are arranged in accordance with the system of Engler and Prantl as shown in the Check List of Flowering Plants and Ferns of the Transvaal and Swaziland, by Mr. J. Burtt-Davy, F.L.S., F.R.S.S.Afr., \& Mrs. R. L. Pott, 1911.

With regard to the lower cryptogams, of which unfortunately there are but few records, I have endeavoured to follow the arrangement given in Dr. Strasburger's Text Book of Botany, 1903, English edition.

So far as the information is available, each plant record gives the following particulars in the order shown, but very few of the records are complete in each detail.

(1) A genus number is placed in left margin opposite the first species of each genus. This corresponds to the number of that genus given in the Genera Siphonogamarum. The cryptogams (Asiphonogama) have no genus numbers in this RECORD. 
(2) The first species of each genus is given its full generic and specific names, with authority. For second and following species the genus name is expressed by its initial letter.

(3) A limited number of synonyms are given in italics within brackets next to the accepted names. They are also shown in the Alphabetical List in italics. Only such synonyms have been inserted as seem necessary to save confusion and facilitate study; as, for instance, where two collectors record the same plant under different names, and where changes of nomenclature have taken place since the publication of the earlier South African Floras.

(4) A considerable number of additional synonyms have been necessitated in order to keep the RECORD uniform with Torre \& Harms' Genera Siphonogamarum. In no instance does this imply any expression of opinion on my part as to which is the more correct classification. The following example will show how a synonym of this kind appears. The genus Chailletia is included by Torre \& Harms under the genus Dichapetahum. Therefore, "Chailletia cymosa, Hook.," is recorded thus :4283-Dichapetalum cymosum (Hook.), Torre \& Harms.

(Chailletia cymosa, Hook.),

and is indexed under both names.

(5) Following the binomial, and synonyms if any, on a new line references are given to any works where descriptions of the species may be found. These references are not necessarily, or even generally, to original descriptions, but to such publications as are likely to be accessible to South African students.

(6) Finally, under each species are recorded such details as are known of its collection, in the following order: Locality, altitude, month of collection, collector's name, and herbarium number. Noте.-A locality is named once only in each record, e.g. "Matopos, Nov. Marloth, 3588; Rogers, 5185." This means that Dr. Marloth's No. 3588 was taken in the Matopo Hills in November, and Archdeacon Rogers' 5185 was also taken in the Matopos, date unknown.

The names of between forty and fifty collectors appear in the RECORD. Many of these put themselves to great trouble to make and send me duplicate copies of their lists of identified plants. The records of others I have obtained from various publications. The following is a list of the names that are most frequent in the RECORD, together with the abbreviated forms used :-

Rand.-Dr. R. F. Rand, F.L.S., formerly of Rhodesia.

Allen.-Mr. C. E. F. Allen, formerly in charge of Forests for Rhodes:an Government.

Oates.-The late Frank Oates, F.R.G.S. 
Monro.-Mr. C. F. H. Monro, M.A., of Victoria.

Rogers.-Archdeacon Rogers, F.L.S., formerly of Rhodesia.

Swyn.-Mr. C. F. M. Swynnerton, F.L.S., of Melsetter.

Gibbs.-Miss L. S. Gibbs, F.L.S.

Eyles.-Mr. F. Eyles, F.L.S., of Mazoe.

Galpin.-Mr. E. E. Galpin, F.L.S., of Cape Province.

Marloth.-Dr. R. Marloth, Ph.D., M.A., F.R.S.S.Afr., of Capetown.

Kolbe.-Rev. F. C. Kolbe, B.A., D.D., of Capetown.

Flanagan.-Mr. H. G. Flanagan, of Cape Province.

Gardner.-Rev. Fr. Gardner, S.J., of Bulawayo.

Cecil.-Hon. Mrs. Evelyn Cecil.

Jeffreys.-Mr. Mervyn Jeffreys, of Bulawayo.

Craster.-Mrs. W. S. Craster, of Salisbury.

Chubb.-Mr. E. C. Chubb, F.Z.S., formerly Curator of Bulawayo Museum.

Bennett.-Mrs. Bennett, of Umtali.

Sr. Phil.-Sister Philomela, of Gwelo.

Noble.-Mr. H. J. Noble, of Gwanda.

Engler.-Dr. Engler, of Berlin.

Govt. Herb.-Herbarium of Government Botanist in Department of Agriculture, Satisbury.

It must be understood that where altitudes are given they are necessarily approximate. Localities also indicate the neighbourhood rather than a precise spot; e.g. "Salisbury" means the neighbourhood of Salisbury.

The following publications have been used in compiling the RECORD wherein references to them will be found in the abbreviated forms shown below :-

Harv. Gen. S.A. Pl.-The Genera of South African Plants, by W. H. Harvey, 2nd Edn., 1868.

Fl. Cap.-Flora Capensis.

Fl. Trop. Afr.-Flora of Tropical Africa.

Cat. Afr. Pl. Welw.-Catalogue of Dr. Welwitsch's African Plants.

Ferns of S.A.-Ferns of South Africa, by T. R. Sim, F.L.S., 1892 (illustrated).

For. Fl. Cape.-The Forests and Forest Flora of Cape Colony, by T. R. Sim, F.L.S., 1906 (illustrated).

For. Fl. P. E. Afr.-Forest Flora and Forest Resources of Portuguese East Africa, by T. R. Sim, F.L.S., 1909 (illustrated).

Nat. Pl.--Natal Plants, by J. M. Wood, A.L.S. (illustrated). The references are given to plate numbers.

Orchids of S.A.-Orchids of South Africa, by Dr. Harry Bolus, F.L.S. (illustrated). 
Journ. Bot.-Journal of Botany.

Journ. Linn. Soc. Bot.-Journal of Linnean Society, Botany.

Kew Bull.-Kew Bulletin.

Trans. S.A. Phil. Soc.-Transactions of South African Philosophical Society.

Trans. R. S. S. Afr.-Transactions of Royal Society of South Africa.

Pro. Rhod. Sc. Assn.-Proceedings of Rhodesia Scientific Association.

Rhod. Agric. Journ.-The Rhodesia Agricultural Journal.

I am under great obligations to many friends for advice and assistance in preparing this record of Rhodesian plants, and wish particularly to acknowledge the following :-

Dr. Rendle and Staff of British Museum for identifying most of my collections.

The late Professor P. MacOwan, of Cape Town, for advice and identifications.

The late Dr. Harry Bolus, of Cape Town, for valuable suggestions, and for continuous and unwearied help in identifying plants and furnishing me with lists of Rhodesian collections made by other botanists.

Mr. T. R. Sim, for identifying ferns, etc.

Dr. Marloth, for help in identification and general encouragement.

Archdeacon Rogers, for adding a number of localities as well as of species to the present list. These additions, interpolated through the kindness of Dr. E. P. Phillips, of the Botanical Department of the South African Museum, are marked in the list with an asterisk. Dr. Phillips has besides looked through the proofs.

Mr. Swynnerton, for the trouble he took to annotate and correct for my benefit the 240 pages descriptive of his Gazaland collections in the Linnean Journal of October, 1911.

Dr. Rand, Miss Gibbs, Mrs. Craster, and Messrs. Allen, Monro, Jeffreys, Chubb, and Father Gardner for sending me lists of their collections.

Mr. J. Burtt-Davy for help and advice.

Also Messrs. Mundy \& Walters, Botanists to the Rhodesian Government, have lent me willing aid and placed their herbarium at my disposal.

I am aware of many deficiencies in this RECORD, but venture to think it should be published without further delay in the belief that it will be useful to beginners, and in the hope that other workers in this field will point out its chief faults, contribute additions, and make suggestions for the improvement of any later edition if called for.

Mazoe, Rhodesta, 1915.

FRED. EYLES. 
Group I.-EMBRYOPHYTA ASIPHONOGAMA.

Division I.-THALLOPHYTA.

Class IV.-CYANOPHYCEAE.

Nostoc commune, Vaucher.

Victoria Falls, Rain Forest, etc. 3000 ft. Sep. Gibbs, 200.

Class XI.-CHARACEAE.

Chara capensis, E. Mey.

Mazoe, Tatagura River, 4300 ft. July, Eyles, 384.

Nitella hyalina, $A g$.

Matopos, Sep. Gibbs, 311.

\section{CLASS XII.-HYPHOIMYCETES.}

Cronartium bresadoleanum, Henn. var. eucleae, Henn.

Bulawayo, $4400 \mathrm{ft}$. Nov. Eyles, 1253. Parasitic on leaf of Euclea Kellau, Hochst.

Polystictus sanguineus, Meyer.

See Cat. Afr. Pl. Welw. ii. 467, P. sanguineus, Fr.

Victoria Falls, Sep. Gibbs, 165, on dead wood.

Hymenochæte rubiginosa, Lév.

Cat. Afr. Pl. Welw. ii. 471.

Victoria Falls, Sep. Gibbs, 262, on dead wood.

Gibbera tinctoria, Massee.

Kew Bull., 1911, 226.

Hunyani Valley, Allen, 734, on leaves of Monotes glaber, Sprague.

Class XIII.-LICHENES.

Leptogium sp.

Bulawayo, Jan. Eyles, 1179.

Parmelia sp.

Matopos, Nov. Eyles, 1148.

Physcia flavicans, $D C$.

Chirinda, 3800 ft. Swyn. 883, on trees. 
Usnea barbata, Ach.

Cat. Afr. Pl. Welw. ii. 396.

Matopos, March, Eyles, 1023.

U. longissima, Ach.

Cat. Afr. Pl. Welw. ii. 397.

Chirinda Forest, 3800 ft. Swyn. 430, hanging from tree branches; Chimanimani Mts. 6500 ft. Swyn. 617.

\section{Division II.-BRYOPHYTA.}

\section{Class I.-HEPATICAE.}

Riccia fluitans, $L$.

Matopos, Sep. Gibbs, 318, in stréams.

Fimbriaria marginata Nees.

Mazoe, Sep. Eyles, 414.

Marchantia polymorpha, $L$.

Matopos, Nov. Eyles, 1181.

Plagiochasma sp.?

Matopos, fr. Oct. Gibbs, 324.

Madotheca capensis, Gottsche.

Chirinda, 3800 ft. Swyn. 812, on trees.

\section{Class II.-MUSCI.}

Amblystegium varium, Lindb.

Mazoe, Aug. 4600 ft. Eyles, 402, on stones by stream.

\section{Bartramia sp.}

Matopos, March, Eyles, 1050.

Catharinea androgyna, C. Muell.

Mt. Pene, 7000 ft. Swyn. 6021.

\section{Hypnum sp.}

Matopos, March, Eyles, 1026.

Octodiceras julianum, Brid.

Mazoe, 4600 ft. Aug. Eyles, 401, submerged.

\section{Orthotrichum sp.}

Matopos, March, Eyles, 1052.

\section{Racopilum sp.}

Victoria Falls, 3000 ft. May, Eyles, 112, in Rain Forest.

Ulota crispa, $B r$. \& Schimp.

Matopos, March, Eyles, 1048. 


\section{Division III.--PTERIDOPHYTA.}

\section{Class I.-FILICALES.}

Order I.-FILICALES LEPTOSPORANGIATAE.

Family I.-HYMENOPHYLLACEAE, Sadebeck.

\section{Hymenophyllum gracile, Bory.}

Ferns of S.A. 48.

Mt. Pene, 7000 ft. Swyn. 6016 ; Mazoe, 5100 ft. April, Eyles, 359.

\section{Family II.-CYATHEACEAE, Diels.}

Cyathea Dregei, Kunze.

Ferns of S.A. 57.

Matopos, 4800 ft. May, Eyles, 72 ; Oct. Gibbs, 292 ; Penhalonga, Bennett; Umtali, Holland; Mt. Pene, 6500-7000 ft. Swyn. 816.

C. manniana, Hook.

Mt. Pene, $7000 \mathrm{ft.} \mathrm{Swyn.} 6030$.

C. Thomsoni, Bak.

Journ. Bot. 1881, 180.

Chirinda, 3800 ft. Swyn. 817.

\section{Fasilly III.-POLYPODIACEAE, Diels.}

Aspidium aculeatum, Swartz, var. pungens, Klf.

Ferns of S.A. 166.

Umtali, Holland \& Bennett.

A. ammifolium, Poir.

Mt. Pene, 6500-7000 ft. Swyn. 878, 6031, 6032.

Nephrodium albo-punctatum, Desv.

Ferns of S.A. 173; Cat. Afr. Pl. Welw. ii. 272.

Mazoe, 5200 ft. April, Eyles, 337; Sebakwe, 4110 ft. Dec. Eyles, 390 ; Matopos, Oct. Gibbs, 210 ; Chimanimani Mts. 7000 ft. Swyn. 857; Salisbury, Darling, Holland \& Hole; Umtali, Bennett.

N. athamanticum, Hook.

Ferns of S.A. 183 ; Cat. Afr. Pl. Welw. ii. 272.

Mazoe, Holland; and at 4400 ft. Jan. Eyles, 562 ; Umtali, Bennett; Chirinda, 3800 ft. Swyn. 875, 876; Lusitu Riv. 3000 ft. Sivyn. $875 a$. 
N. bergianum, Baker.

Ferns of S.A. 175.

Matopos, 4700 ft. April \& May, Eyles, 63 ; Mazoe, 4500 ft. Feb. Eyles, 525 ; Umtali, Darling; Penhalonga, Bennett \& Holland; Victoria Falls, Richards; Chirinda Forest, 3700-4000 ft. Swyn. $866,870$.

N. catopteron, Hook.

Ferns of S.A. 185.

Umtali, Bennett \& Holland.

N. cicutarium, Baker. (N. hippocrepis, Desv.)

Ferns of S.A. 187; Cat. Afr. Pl. Welw. ii. 273.

Umtali, Darling, Holland \& Bennett; Chirinda Forest, 3700-4000 ft. Swyn. 823 ; Mt. Pene, 6500-7000 ft. Swyn. 824a.

N. Filix-mas, Rich. var. elongatum, Hook.

Ferns of S.A. 180.

Umtali, Bennett \& Darling; Lomagundi, Darling; Mazoe, $4600 \mathrm{ft}$. Jan. Eyles, 561; Chirinda, 3800 ft. Swyn. 874, 457.

N. inæquale, Hook.

Ferns of S.A. 182.

Lomagundi, Darling; Umtali, Holland.

N. mauritianum, Fèe.

Ferns of S.A. 176.

Lomagundi, Darling; Mazoe, Holland; Victoria Falls, Holland.

N. molle, Desv.

Ferns of S.A. 177; Cat. Afr. Pl. Welw. ii. 273.

Victoria Falls, Rain Forest, 3000 ft. May, Eyles, 95; Allen, 2; Rogers, 5053; Chirinda Forest, 3700-4000 ft. Swyn. 864, 865, 867, 870 ; Chirinda, 3800 ft. Swyn. 868, 869 ; Umtali, Engler; Matabeleland, Oates.

var. violaceum, (Link) Mett.

Ferns of S.A. 177, a note under N. molle.

Victoria Falls, Palm Kloof, Engler.

N. Thelypteris, Desv.

Ferns of S.A. 179 ; Cat. Afr. Pl. Welw. ii. 272.

Matopos, 4600 ft. May, Eyles, 69 ; Rogers, 5197; Umtali, Darling \& Bennett; Mazoe, Darling; Penhalonga, Holland; Rusapi, Engler.

N. Thelypteris, Desv., var. squamuligera, Schlechtend.

See Ferns of S.A. 180.

Chirinda, 3800 ft. Swyn. 858, 859a.

N. unitum, $R . B r$.

Ferns of S.A. 178; Cat. Afr. Pl. Welw. ii. 273.

Victoria Falls, on island, Sep. Gibbs, 151 ; \& Holland. 
Nephrolepis cordifolia, Presl.

Trans. S.A. Phil. Soc. xvi. 288.

Matabeleland, Oates; Umtali, Darling ; Victoria Falls, Rain Forest, Sep. Gibbs, 155 ; Holland, Richards \& Engler; Chirinda, 37004000 ft. Swyn. 856.

N. exaltata, Schott.

Trans. S.A. Phil. Soc. xvi. 289 ; Cat. Afr. Pl. Welw. ii. 274.

Matabeleland, Oates; Victoria Falls, Rain Forest, 3000 ft. May, Eyles, 116 ; Sep. Gibbs, 148; Allen, 1 \& Engler ; Umtali, Darling.

Davallia concinna, Schrad.

Odzani River Valley, Umtali, Teague, 244.

D. Hollandii, Sim.

Trans. S.A. Phil. Soc. xvi. 274.

Umtali, Holland \& Bennett.

D. thecifera, H.B.K.

Cat. Afr. Pl. Welw. ii. 265.

Mt. Pene, 7000 ft. Swyn. 6018.

Lomaria attenuata, Willd.

Ferns of S.A. 117.

Umtali, Darling \& Bennett ; Chimanimani Mts. 7000 ft. Swyn. 835a; Mt. Pene, 7000 ft. Swyn. 6023, 6024.

L. Boryana, Willd.

Ferns of S.A. 123 ; Cat. Afr. Pl. Welw. ii. 269.

Umtali, Bennett \& Darling; Chimanimani Mts. 7000 ft. Swyn. 835 ; Mt. Pene, 7000 ft. Swyn. 6022.

L. procera, Spreng.

Ferns of S.A. 122.

Chirinda, 3700-4000 ft. Swyn. 832.

L. punctulata, Kze.

Ferns of S.A. 118.

Mazoe, 5000-5200 ft. April, Eyles, 342 ; Victoria Falls, Rogers, 5546.

Asplenium adiantum-nigrum, $L$.

Ferns of S.A. 148.

Umtali, Darling.

A. anisophyllum, $K z e$.

Ferns of S.A. 142.

Umtali, Bennett \& Darling.

A. aspidioides, $S c h l$.

Ferns of S.A. 162.

Umtali, Darling \& Holland.

A. cicutarium, $S w$.

Ferns of S.A. 155.

Umtali, Darling \& Bennett. 
var. abyssinicum, Baker.

Chirinda Forest, 3700-4000 ft. Swyn. 819.

A. dregeanum, $K z e$.

Ferns of S.A. 156.

Umtali, Darling.

A. erectum, Bory.

Ferns of S.A. 136 ; Cat. Afr. Pl. Welw. ii. 270.

Victoria Falls, Palm Kloof, Engler; Chirinda Forest, 3700-4000 ft. Swyn. 827.

var. erectum, Bory.

Ferns of S.A. 138.

Umtali, Bennett.

var. lobatum, (P.\& R.) Sim.

Ferns of S.A. 139.

Umtali, Bennett \& Holland.

var. lunulatum, (Sw.) Sim.

Ferns of S.A. 137.

Victoria Falls, Holland.

A. Eylesii, Sim.

Mazoe, 4500 ft. Jan. Eyles, 564.

A. filix-fœmina, Bernh .

Ferns of S.A. 160.

Penhalonga Forest, Bennett; Umtali, Holland.

A. furcatum, Thunb. (A. adiantoides, Lam.)

Ferns of S.A. 152 ; Cat. Afr. Pl. Welw. ii. 270.

Salisbury, Darling \& Hole; Umtali, Holland \& Bennett; Matopos, Sep. Gibbs, 28; Victoria Falls, Garbutt; Mazoe, 4500 ft. Dec. Eyles, 477 ; Chirinda Forest, 3700-4000 ft. Swyn. 845; Chimanimani Mts., 7000 ft. Swyn. 846a; Melsetter, 6000 ft. Swyn. 808; Mt. Pene, 7000 ft. Swyn 6026; Odzani River Valley, Umtali, Teague, 161.

var. tripinnatum, Baker.

Ferns of S.A. 154.

Umtali, Bennett \& Darling; Mt. Pene, 7000 ft. Swyn. 846, 6027.

A. gemmiferum, Schr.

Ferns of S.A. 145.

Umtali, Bennett \& Holland; Chirinda Forest, 3700-4000 ft. Swyn. 402, 853.

var. flexuosum, Schr.

Ferns of S.A. 145.

Umtali, Bennett. 
var. laciniatum, Mett.

See note Ferns of S.A. 147.

Chirinda Forest, 3700-4000 ft. Swyn. 844, 844a.

A. lætum, Swartz.

Chirinda Forest, 3700-4000 ft. Swyn. 840, 840 b.

A. Mannii, Hook.

Trans. S.A. Phil. Soc. xvi. 285.

Mt. Pene, 7000 ft. Swyn. 6015; Chipete Forest, 3800 ft. Swyn. 427.

A. monanthemum, $L$.

Ferns of S.A. 135.

Umtali, Bennett. Not common.

A. protensum, Schraa?.

Ferns of S.A. 141.

Matopos, 5000 ft., March, Eyles, 1022 ; Umtali, Holland \& Bennett ;

Chirinda Forest, 3700-4000 ft. Swyn. 879, 880.

A. resectum, Smith.

Cat. Afr. Pl. Welw. ii. 270.

Chirinda Forest, 3700-4000 ft. Swyn. 840a, 841.

A. rutæfolium, Kze.

Ferns of S.A. 158.

Umtali, Bennett \& Holland.

A. Sandersoni, Hook.

Ferns of S.A. 132.

Umtali, Bennett.

A. Schimperi, $A$. $B r$.

Ferns of S.A. 161.

Salisbury, Darling.

A. serra, Langs. var. natalensis, Baker.

Ferns of S.A. 144.

Chimanimani Mts. 6500 ft. Swyn. 851.

A. Thunbergii, Kze.

Ferns of S.A. 157.

Chirinda Forest, 3700-4000 ft. Swyn. 806; Chipetzana source, 4000 ft. Swyn. 807; Mt. Pene, 7000 ft. Swyn. 6017.

A. trichomanes, $L$.

Ferns of S.A. 133.

Lomagundi, Sinoia Cave and Hunyani River, Hole.

A. varians, $H k$. \& $G r$.

Ferns of S.A. 140.

Umtali, Darling.

Gymnogramme argentea, Mett. (G. rosea, Desv.)

Ferns of S.A. 214 ; Cat. Afr. Pl. Welw. ii. 276.

Chimanimani Mts., 7000 ft. Swyn. 677. 
var. aurea, Mett.

Ferns of S.A. 214, 5.

Mazoe, $5200 \mathrm{ft}$. April, Eyles, 340.

G. cordata, Schlecht.

Ferns of S.A. 210 ; Cat. Afr. Pl. Welw. ii. 276.

Bulawayo, 4600 ft. Feb. Eyles, 392 ; Lomagundi, Darling; Salisbury, Darling; Matopos, 4800 ft. March, Eyles, 1021 ; Gibbs, Sep. 29. var. namaquensis, $P . \& R$.

Ferns of S.A. 211, 2.

Salisbury, Hole.

G. lanceolata, Hook.

Ferns of S.A. 215.

Umtali, Bennett; Chirinda, 3800 ft. Swyn. 814 ; Mt. Pene, 7000 ft. Swyn. 6020.

G. leptophylla, Desv.

Ferns of S.A. 212.

Mazoe, 4500 ft. Jan. Eyles, 563.

Vittaria isœtifolia, Bory. (V. lineata, Sw.)

Ferns of S.A. 216.

Chimanimani Mts. 7000 ft. Swyn. 802.

Pellæa burkeana, Baker. (P. dura, Hook.)

Ferns of S.A. 105 ; Cat. Afr. Pl. Welw. ii. 267.

Mazoe, 5000 ft. April, Eyles, 263; Darling; Salisbury, Darling \& Hole; Umtali, Holland \& Bennett, Odzani River Valley, Teague, 70 .

P. calomelanos, Link.

Ferns of S.A. 104 ; Cat. Afr. Pl. Welw. ii. 267.

Matopos, 4700 ft. April, Eyles, 394; Holland; Salisbury, Hole ; Umtali, Bennett; Odzani River Valley, Teague, 67; Chirinda, 3600 ft. Swyn. 850 ; Matabeleland, Fry.

P. consobrina, Hook.

Ferns of S.A. 97.

Mazoe, 5000 ft. April, Eyles, 357; Darling \& Bennett; Umtali, Darling \& Bennett; Odzani River Valley, Teague, 68; Chimanimani Mts., 7000 ft. Swyn. 852; Mt. Pene, 7000 ft. Swyn. 6028 .

P. Doniana, Hook.

Trans. S.A. Phil. Soc. xvi. 279.

Umtali, Bennett.

P. geraniæfolia, Fèe. (Cheilanthes Kirkii, Hook.)

Ferns of S.A. 92 ; Cat. Afr. Pl. Welw. ii. 266.

Matopos, 4800 ft. March, Eyles, 1044; Gibbs, Sep. 291; Victoria, Zimbabwe, Holland, Monro, 732 ; Umtali, Bennett. 
P. hastata, Link.

S.A. Ferns, 101 ; Cat. Afr. Pl. Welw. ii. 267.

Matopos, 5000 ft. March, Eyles, 1018, April, 312 ; Sep. Gibbs, 89 ;

Salisbury, Hole; Umtali, Bennett; Mazoe, Holland; Victoria, Holland.

var. glauca, $\mathrm{Sim}$.

Ferns of S.A. 102.

Salisbury, Darling, Hole \& Holland; Mazoe, Darling \& Holland; Matabeleland, Fry.

var. macrophylla, Hook.

Ferns of S.A. 102.

Chirinda Forest, 3600-4000 ft. Swyn. 800; Victoria Falls, Garbutt.

P. pectiniformis, Baker.

Ferns of S.A. 95 ; Cat. Afr. Pl. Welw. ii. 267.

Matopos, Oct. Gibbs, 294 ; Mazoe, 5000 ft. March, Eyles, 250.

Notochlæna Buchanani, Baker.

Ferns of S.A. 207.

Matopos, Sep. Gibbs, 70.

N. inæqualis, $K z e$.

Ferns of S.A. 206 ; Cat. Afr. Pl. Welw. ii. 276.

Matopos, 5000 ft. March, Eyles, 1020 ; Salisbury, Holland \& Darling ; Mazoe, 5200 ft. April, Eyles, 339.

Chielanthes Bolusii, Baker.

Ferns of S.A. 89 ; Trans. S.A. Phil. Soc. xvi. 277.

Mazoe, 5000 ft. March, Eyles, 247 ; Umtali, Holland.

C. farinosa, Kaulf.

Trans. S.A. Phil. Soc. xvi. 278 ; Cat. Afr. Pl. Welw. ii. 267.

Victoria Falls, Rain Forest, 3000 ft. May, Eyles, 121, 147 ; Sep. Gibbs, 6 ; Galpin, 7053 ; Allen, 13 ; Rogers, 5013, 5545 ; Engler, Richards \& Holland; Matabeleland, Oates.

C. hirta, Swartz.

Ferns of S.A. 83 ; Cat. Afr. Pl. Welw. ii. 266.

Matopos, 5000 ft. May, Eyles, 76; Sep. Gibbs, 90 ; Engler ; Bulawayo, 4500 ft. Feb. Eyles, 393 ; Umtali, Bennett ; Odzani River Valley, Teague, 160.

C. multifida, Swartz.

Ferns of S.A. 87.

Matopos, Sep. Gibbs, 69 ; 5000 ft. March, Eyles, 1019.

Hypolepis anthriscifolia, Presl.

Ferns of S.A. 76.

Chirinda Forest, 3700-4000 ft. Swyn. 820. 
H. bergiana, Hook.

Ferns of S.A. 78.

Chimanimani Mts., 7000 ft. Swyn. 821.

H. Schimperi, Sim.

Trans. S.A. Phil. Soc. xvi. 276.

Mazoe, 4800-5300 ft. Dec. Eyles, 395; Darling \& Holland; Salisbury, Darling, Holland \& Hole.

Crystopteris fragilis, Bernh.

Eerns of S.A. 66.

Lomagundi, Hole.

Adiantum æthiopicum, $L$.

Ferns of S.A. 73.

Mazoe, 5100 ft. April, Eyles, 328; Holland; Salisbury, Holland; Umtali, in hills, Bennett; Matabeleland, Oates.

A. capillus-Yeneris, $L$.

Ferns of S.A. 70 ; Cat. Afr. Pl. Welw. ii. 266.

Bulawayo, 4500 ft. May, Eyles, 68; Victoria Falls, Rain Forest, Sep. Gibbs, 20; May, Eyles, 113, 126 ; Allen, 11; Rogers, 5547; Engler \& Holland; Umtali, Bennett \& Darling; Mazoe, Darling.

A. caudatum, $L$.

Ferns of S.A. 69.

Sebakwe, 4000 ft., Nov. Eyles, 389 ; Victoria Falls, Sep. Gibbs, 180 ; Rogers, 5052; Allen, 17 ; Engler, Richards \& Holland; Umtali, Darling \& Bennett; Mazoe, Darling; Matopos, Gibbs, 316.

A. lunulatum, Burm.

Ferns of S.A. 70 ; Cat. Afr. Pl. Welw. ii. 265.

Victoria Falls, Rain Forest, 3000 ft. May, Eyles, 127; Allen, 9; Rogers, 5376, 5549; Richards \& Holland; Mazoe, Darling; Umtali, Bennett; Matabeleland, Oates.

A. Oatesii, Baker.

In "Matabeleland and Victoria Falls," by Frank Oates, appendix by A. Rolfe, ed. i. p. 369 ; Ferns of S.A. 70.

Victoria Falls, Sep. Gibbs, 317; Allen, 20 ; Lomagundi, Darling ; Matabeleland, Oates.

\section{Actiniopteris radiata, Link.}

Ferns of S.A. 163 ; Harv. Gen. S.A. Pl. 465 ; Cat. Afr. Pl. Welw. ii. 271. Mazoe, 4400-4600 ft. March, Eyles, 259; Victoria Falls, Sep. Gibbs, 33; Allen, 91 ; Garbutt; Matopos, Engler \& Gibbs; Salisbury, Darling \& Hole; Umtali, Bennett; Odzani River Valley, Umtahi, Teague, 63.

Didymochlæna lunulata, Desv. (D. simosa, Desv.)

Ferns of S.A. 164; Cat. Afr. Pl. Welw. ii. 271.

Mt. Pene Forest, 6500-7000 ft. Swyn. 815; Umtali, Bennett. 
Pteris aquilina, $L . \quad$ (Pteridium aquilinum, Kuhn.)

Ferns of S.A. 113 ; Cat. Afr. Pl. Welw. ii. 268 ; Harv. Gen. S.A. Pl. 464.

Matopos, 4800 ft. May, Eyles, 79 ; Umtali, Darling; Odzani River Valley, Teague, 251 ; Salisbury, Hole; Mt. Hampden, Holland (a glabrous var.) ; Victoria Falls, Palm Kloof, Engler.

var. lanuginosa, Hook.

Ferns of S.A. 114, included under type ; Cat. Afr. Pl. Welw. ii. 268.

Chirinda, $2800 \mathrm{ft}$. Swyn. 401 in part; Mt. Pene, $7000 \mathrm{ft}$. Swyn. 6029 ; throughout Melsetter Dist., Swyn.

P. atrovirens, Willd.

Cat. Afr. Pl. Welw. ii. 268.

Victoria Falls, Palm Kloof, Engler.

P. brevisora, Baker.

Chirinda Forest, 3700-4000 ft. Swyn. 871.

P. cretica, $L$.

Ferns of S.A. 107; Cat. Afr. Pl. Welw. ii. 268.

Mazoe, 4900 ft. Dec. Eyles, 498; Holland ; Chirinda Forest, $3700-$ 4000 ft. Swyn. 831 ; Umtali, Bennett; Salisbury, Hole.

P. flabellata, Thunb. (P. arguta, Sw.)

Ferns of S.A. 110 ; Cat. Afr. Pl. Welw. ii. 268.

Victoria Falls, Rogers, 5556; Penhalonga, Holland; Umtali, Bennett.

P. longifolia, $L$.

Ferns of S.A. 106; Cat. Afr. Pl. Welw. ii. 267.

Bulawayo, 4500 ft. May, Eyles, 66; Mazoe, Darling, Holland \& Bennett; Umtali, Darling, Holland \& Bennett.

P. quadriaurita, Retz.

Ferns of S.A. 108 ; Cat. Afr. Pl. Welw. ii. 268.

Victoria Falls, Rain Forest, 3000 ft. May, Eyles, 94; Allen, 181; Rogers, 5048; Gibbs; Richards; Matopos, Oct. Gibbs, 223 ; May, Eyles, 149; Umtali, Darling; Bennett; Lomagundi, Darling; Salisbury, Holland; Chirinda Forest, 3700-4000 ft. Swyn. 872 ; Matabeleland, Oates.

Polypodium africanum, Mett.

Ferns of S.A. 203.

Mazoe, 4500 ft. Jan. Eyles, 388; Chirinda Forest, 3700-4000 ft. Swyn. 425; Chipete Forest, 3800 ft. Swyn. 425ı; Umtali, Bennett; Holland.

P. incanum, Swartz.

Ferns of S.A. 194.

Victoria, Zimbabwe, Holland; Chirinda Forest, 370J-40J) ft. Swyn. 809. 
P. irioides, Lam.

Ferns of S.A. 204 ; Cat. Afr. Pl. Welw. ii. 275.

Macequece, Holland; Umtali, Bennett.

P. lanceolatum, L. (P. lepidotum, Willd.)

Ferns of S.A. 201.

Umtali, Bennett; Melsetter, $6000 \mathrm{ft}$. Swyn. 811, epiphytic on Brachystegia Randii and other trees; Chipete Forest, $3800 \mathrm{ft}$. Swyn. 812 ; Chimanimani Mts. 7000 ft. Swyn. 813 ; Mt. Pene, 7000 ft. Swyn. 6008, 6019; Odzani River Valley, Umtali, Teague, 162.

B. normale, Don.

Ferns of S.A. 199.

Umtali, Bennett.

P. obtusilobum, Desv.

See Ferns of S.A. 174, 5. (Sim doubts validity of this species and does not distinguish it from Nephrodium bergianum, Baker.)

Victoria Falls, Allen, 3 ; Gwaai River, 3400 ft. Allen, 229.

P. phymatodes, $L$.

Ferns of S.A. 196; Cat. Afr. Pl. Welw. ii. 275.

Umtali, Bennett; Lusitu River, 2500 ft. Swyn. 661, 884.

P. proliferum, Prest.

Ferns of S.A. 192.

Hunyani Riv. Darling; Mazoe, Holland; Umtali, Bennett.

P. unitum, Hook.

Ferns of S.A. 193.

Umtali, Bennett.

Acrostichum latifolium, Swartz.

Ferns of S.A. 220.

Umtali, Bennett; Mt. Pene, 7000 ft. Swyn. 6009.

A. lineare, Fèe.

Mt. Pene, 7000 ft. Swyn. 6014.

A. viscosum, $S w$.

Ferns of S.A. 221.

Chimanimani Mts. 7000 ft. Swyn. 810.

Platycerium alicorne, Desv.

Trans. S.A. Phil. Soc. xvi. 293.

Macequece, Holland; Umtali, Bennett.

Family IV.-GLEICHENIACEAE, Diels.

Gleichenia dichotoma, Willd.

Ferns of S.A. 45.

Umtali, Bennett. 
G. polypodioides, Smith.

Ferns of S.A. 43 ; Cat. Afr. Pl. Welw. ii. 263.

Mazoe, 5200 ft. April, Eyles, 341 ; Chimanimani Mts. 6500 ft. Swyn. 676.

G. umbraculifera, T. Moore.

Ferns of S.A. 44.

Melsetter, 6000 ft. Swyn. 613 ; Lusitu Hills, 5000 ft. Swyn. 1497.

FAmily V.-SCHIZAEACEAE, Diels.

Aneimia anthriscifolia, Schrad.

Trans. S.A. Phil. Soc. xvi. 294.

Umtali, Bennett \& Holland.

A. tomentosa, $S w$.

Odzani River Valley, Umtali, Teague, 69.

Mohria caffrorum, Desv.

Ferns of S.A. 233.

Matopos, 4800 ft. March, Eyles, 1042, May, 128; Gibbs, Oct. 235; Melsetter, 6000 ft. Swyn. 611 ; Mazoe, Eyles; Umtali, Bennett, Odzani River Valley, Teague, 140; Darling.

M. lepigera, Baker.

Chimanimani Mts. $7000 \mathrm{ft.} \mathrm{Swyn.} \mathrm{611a.}$

Lygodium Brycei, Baker.

Kew Bull. 1901, 138.

Mashonaland, Bryce, nr. Portuguese border, drift of Renie Riv.

L. Kerstenii, Kuhn. (L. subulatum, Bojer.)

Trans. S.A. Phil. Soc. xvi. 296.

Penhalonga, Holland; Umtali, Bennett; South Melsetter District, abundant in wooded kloofs.

\section{Family VI.-OSMUNDACEAE, Diels.}

\section{Osmunda regalis, $L$.}

Ferns of S.A. 227 ; Cat. Afr. Pl. Welw. ii. 278.

Matopos, 4600 ft. April, Eyles, 56 ; Rogers, 5193 ; Marloth ; Chirinda, 3700 ft. Swyn. 828 ; Chipetzana source, 4000 ft. Swyn.; Umtali, Bennett, Odzani River Valley, Teague, 66; Darling; Mazoe, Hole; Salisbury, Holland.

Todea africana, Willd. (T. barbara, Moore.)

Ferns of S.A. 228.

Chimanimani Mts. 7000 ft. Swyn. 830. 
FAmily VII.-MARSILIACEA, Sadebeck.

Marsilia biloba, Willd.

Ferns of S.A. 258.

Gwelo, in vlei, Gardner, 28.

M. capensis, $A$. $B r$.

Ferns of S.A. 259.

Matopos, fr. Oct. Gibbs, 289.

M. macrocarpa, Presl. var. lobata, Sim.

Ferns of S.A. 259 (type).

Pasipas Mt. 4500 ft. June, Eyles, 26.

\section{ORder II.--MARATTIALES. \\ FAmily VIII.-MARATTIACEAE, Bitter.}

\section{Marattia fraxinea, Smith.}

Ferns of S.A. 235 ; Cat. Afr. Pl. Welw. ii. 278.

Chirinda Forest, 3700-4000 ft. Swyn. 826; Mt. Pene Forest, 6500-7000 ft. Swyn. 826a, 6025; Umtali, Darling; Bennett; Penhalonga, Holland.'

ORder III.-OPHIOGLOSSALES.

Family IX.-OPHIOGLOSSACEAE, Bitter.

Ophioglossum reticulatum, $L$.

Ferns of S.A. 238 ; Cat. Afr. Pl. Welw. ii. 279.

Mazoe, 4800 ft. Jan. Eyles, 559.

0. vulgatum, $i$.

Ferns of S.A. 237.

Khami, Nov. Marloth, 3354 ; Matopos, Marloth.

\section{Crass II.-EQUISETALES. \\ Order I.-EU-EQUISETALES. \\ Family X.-EQUisetaCEAE, Sadebeck.}

\section{Equisetum ramosissimum, Desf.}

Ferns of S.A. 240 ; Cat. Afr. Pl. Welw. ii. 279.

Matopos, 4800 ft. April, Eyles, 151; Umtali, Engler. 


\section{Class III.-LYCOPODIALES.}

\section{Order I.-LYCOPODIALES ELIGULATAE.}

FAmily XI.-LYCOPODIACEAE, Pritzel.

\section{Lycopodium carolinianum, $L$.}

Ferns of S.A. 246.

Matopos, 5000 ft. April, Eyles, 51; Oct. fr. Gibbs, 231.

L. cernuum, $L$.

Ferns of S.A. 244 ; Cat. Afr. Pl. Welw. ii. 263.

Chirinda, 3800 ft. Swyn. 458; Odzani River Valley, Umtali, Teague, 252.

L. clavatum, $L$. var. inflexum, Spreng.

Ferns of S.A. 245.

Mt. Pene, 6500-7000 ft. Swyn. 805.

L. verticillatum, $L$.

Ferns of S.A. 243.

Mt. Pene, 7000 ft. Swyn. 6010 ; Umtali, Holland.

Psilotum triquetrum, Swartz.

Ferns of S.A. 247 ; Harv. Gen. S.A. Pl. 470.

Victoria Falls, Rain Forest, 3000 ft. May, Eyles, 115 ; Engler.

Order II.-LYCOPODIALES LIGULATAE.

Family XII.-SELAGINELLACEAE, Hieron.

Selaginella depressa, A. Braun.

Ferns of S.A. 251.

Matopos, March, Eyles, 1043.

S. imbricata, Spreng.

Victoria Falls, dry edge of gorge, Galpin, 7054; Sep. Gibbs, 179 ; Allen, 90; Engler; April, Eyles, 546.

S. kraussiana, A. Braun.

Ferns of S.A. 252.

Chimanimani Mts. 7000 ft. Swyn. 803; Mt. Pene, 7000 ft. Swyn. 6013 ; Umtali, Darling.

S. rupestris, Spreng.

Ferns of S.A. 250 ; Cat. Afr. Pl. Welw. ii. 262.

Matopos, Sep. Gibbs, 4 ; Oct. Davy; Engler; Chimanimani Mts. 7000 ft. Swyn. $617 a$.

S. sp.

Matopos, 4800 ft. March, Eyles, 1043, cf. S. kraussiana, A. Braun. 


\title{
Group II.-EMBRYOPHYTA SIPHONOGAMA.
}

\author{
Division I.-GYMNOSPERMAE.
}

Class CONIFERAE.

\author{
FAMILY V.-TAXACEAE, Lindl.
}

Genus No.

15-Podocarpus milanjiana, Rendle. (P. Thunbergii, Hook. var. falcata.)

See For. Fl. Cape, 332.

Chimanimani Mts. 7000 ft. male fl. Sep. Swyn. 1962 ; Mt. Pene, $7000 \mathrm{ft}$. Swyn. 6038.

FAmily VI.-PINACEAE, Lindl.

38-Callitris Whytei (Rendle), Torre \& Harms. (Widdringtonia Whytei, Rendle; W. Mahoni, Masters.)

For. Fl. Port. E. Afr. 109.

Chimanimani Mts. 7000 ft. Swyn. 1964; Melsetter, 6000 ft. fr. Sep. Swyn. 1963; Hutchins mentions for Umtali, Melsetter and Inyanga; also see Journ. Bot. 1906, 190 for Umtali and Melsetter.

\section{Division II.-ANGIOSPERMAE.}

\section{Class MONOCOTYLEDONEAE.}

SERIES PANDANALES.

Family VIII.-TYPHACEAE, J. St. Hil.

49-Typha australis, Schumach. \& Thonn.

Fl. Trop. Afr. viii. 135 ; Fl. Cap. vii. 31.

Upper Buzi Riv. and Inyamadzi Riv. Swyn.

\section{Series HELOBIAE.}

Family XI.-POTAMOGETONACEAE, Engl.

\section{8-Potamogeton fluitans, Roth.}

Fl. Cap. vii. 46; Fl. Trop. Afr. viii. 219; Cat. Afr. Pl. Welw. ii. 94. Matopos, $4800 \mathrm{ft}$. April, Eyles, 36. 
Genus No.

P. natans, $L$.

Fl. Cap. vii. 46 ; Harv. Gen. S.A. Pl. 387.

Matopos, streams, general, Sep. Gibbs, 94 ; Victoria Falls, common in river, Sep. Gibbs, 146 ; Salisbury, Rand, 540.

P. pusillum, $L$.

Fl. Cap. vii. 49 ; Fl. Trop. Afr. viii. 222.

Matopos, fl. \& fr., Sep. Gibbs, 190, in streams.

Family XIII.-APONOGETONACEAE, Engl.

65 -Aponogeton sp.

No precise locality, Rand, 217.

FAmily XV.-ALISMACEAE, DC.

80-Burnatia enneandra, Micheli.

Fl. Trop. Afr. vii. 213.

Matabeleland, Holub.

FAMILY XVII.-HYDROCHARITACEAE, Aschers.

86- Hydrilla verticillata, Royle.

Fl. Trop. Afr. vii. 2.

Victoria Falls, abundant in river, Sep. Gibbs, 136; Galpin, 7027.

90-Blyxa sp.

Salisbury, May, Flanagan, 3251, in ponds. "Distinct from

$B$. radicans, Ridl. and probably new." H. Bolus.

95-Ottelia paucifolia, A. Rich.

Victoria Falls, Allen, 14.

0. vesiculata, Ridl.

Fl. Trop. Afr. vii. 7 ; Cat. Afr. Pl. Welw. ii. 2.

Matopos, $4800 \mathrm{ft}$. April, Eyles, 34.

\section{Series GLUMIFLORAE. \\ Family XIX.-GRAMineaE, B. Juss.}

102-Zea Mays, $L$.

Universally cultivated.

109-Imperata arundinacea, Cyr. (I. cylindrica, Beauv.)

Fl. Cap. vii. 320 ; Nat. Pl. ii. 101; Cat. Afr. Pl. Welw. ii. 135.

Salisbury, Mundy; Matabeleland, Jeffreys, 49 ; may be new sp. 
Genus No.

var Thunbergii Hack.

Fl. Cap. vii. 320.

North Melsetter, 4000-5000 ft. Swyn.; Bulawayo, Monro, 982 ;

Marandellas, Engler; Umtali, Engler.

111-Saccharum munroanum, Hack.

Fl. Cap. vii. 321; Nat. Pl. ii. 102 ; Cat. Afr. Pl. Welw, ii. 136.

Matopos, Rogers, 5189.

112-Erianthus teretifolius, Stapf.

Journ. Linn. Soc. Bot. xxxvii. 478.

Victoria Falls, fl. \& fr. Sep. Gibbs, 141 ; Rogers, 5098, 5315 Salisbury, Craster, 66.

E. sp.

Lochard, Govt. Herb. 2094, nr. E. sorghum.

113-Pollinia villosa, Spreng.

Fl. Cap. vii. 325 ; Nat. Pl. ii. 109 ; Cat. Afr. Pl. Welw. ii. 136.

Victoria Falls, Sep. Gibbs, 153 ; Rogers, 5810.

\section{P. sp.}

Salisbury, Craster, 18.

119-Ischæmum fasciculatum, Brongn.

Fl. Cap. vii. 327 ; Cat. Afr. Pl. Welw. ii. 141.

Victoria Falls, Engler.

var. arcuatum, Hack.

Fl. Cap. vii. 327 ; Nat. Pl. ii. 104.

Lusitu Riv. 3000 ft. Swyn. 1652 ; Victoria Falls, Rogers, 5088.

\section{I. spp.}

Salisbury, Craster, 37, 69.

126-Rhytachne, sp.

Salisbury, Craster, 56.

127-Rottboellia compressa, $L$.

Fl. Cap. vii. 329 (var.).

Salisbury, Mundy; Bulawayo, Rogers, 5868.

var. fasciculata, Hack.

Fl. Cap. vii. 329 ; Nat. Pl. ii. 105.

Heaney Jnctn. Jeffreys, 17 ; North Melsetter, 4000-6000 ft. Swyn.

R. exalata, $L$. fil.

Victoria Falls, Rogers, 7076*.

131-Trachypogon polymorphus, Hack.

Fl. Cap. vii. 331 (var.); Nat. Pl. ii. 107 (var.).

Salisbury, Mundy; Victoria Falls, Rogers, 5595; Bulawayo, Rogers, 5885. 
Genus No.

132-Elionurus argenteus, Nees.

Fl. Cap. vii. 332 ; Nat. Pl. ii. 108.

Chirinda, 3700 ft. Jan. Swyn. 1694; Mt. Pene, 6500-7000 ft.

Sep. Swyn. 1648; Somabula, Govt. Herb. 666 ; Umtali, Engler.

var. thymidora, Stapf.

Fl. Cap. vii. 333.

Bulawayo, Rogers, 5882.

134-Andropogon amplectens, Nees.

Fl. Cap. vii. 342 ; Nat. Pl. ii. 116.

Heaney Jnctn. Jeffreys, 8, and 21; Somabula, Govt. Herb. 668.

A. ceresiæformis, Nees.

Fl. Cap. vii. 337 ; Nat. Pl. ii. 112 ; Cat. Afr. Pl. Welw. ii. 145.

Between Lusitu and Nyahodi, 5000 ft. April, Swyn. 1656; Salisbury, Craster, 51.

A. connatus, Chiov.

Bulawayo, Rogers, 5918 ; Victoria Falls, Rogers, 5672.

A. contortus, L. (Heteropogon contortus, Roem. \& Schult.)

Fl. Cap. vii. 350 ; Nat. Pl. ii. 121 ; Cat. Afr. Pl. Welw. ii. 153.

Bulawayo, in vlei, Jeffreys, 16 ; Rogers, 5900 ; Victoria, Monro, 907 ; Syringa, Matabeleland, Govt. Herb. 641, 642 ; Salisbury, Craster, 62.

A. cymbarius, L. (Cymbopogon elegans, Spreng.)

Fl. Cap. vii. 360 ; Nat. Pl. 130 ; Cat. Afr. Pl. Welw. ii. 157.

Nyahodi Riv. 4000 ft. April, Swyn. 1675.

A. eucomis, Nees.

Fl. Cap. vii. 338; Nat. Pl. 113.

Matopos, fl. \& fr. Sep. Gibbs, 23 ; Heaney Jnctn. Jan. Jeffreys, 47.

A. filipendulus, Hochst. (Cymbopogon filipendulus, Rendle.)

Fl. Cap. vii. 362 ; Nat. Pl. 131 ; Cat. Afr. Pl. Welw. ii. 157.

Salisbury, March, Flanagan, 3003 ; Bulawayo, Rogers, 5898 ; 13508; Jeffreys, 69, forma pilosior, Hack.; North Melsetter, 4000-6000 ft. April, Swyn. 1687; Chirinda, 3500 ft. Jan. Swyn. 1640 ; Upper Buzi, 3000-3500 ft. April, Swyn. 985, 988, 990 ; Umtali, Engler.

A. gayanus, Kunth.

See Cat. Afr. Pl. Welw. ii. 148.

Victoria, Monro, 991; Chirinda, 3800 ft. April, Swyn. 967 ; Upper Buzi, 3000-3500 ft. April, Swyn. 984 ; Bulawayo, Rogers, 5865 ; Victoria Falls, Rogers, 5673.

A. gazense (Rendle), Torre \& Harms. (Cymbopogon gazense, Rendle.) Journ. Linn. Soc. Bot. xl. 226.

Chirinda, 3700 ft. Jan. Swyn. 1637. 

Chiov.)

Chirinda, 3500 ft. Jan. Swyn. 1636 ; Upper Buzi, 300()-3500 ft. April, Swyn. 994.

A. halapensis, Brot.

Fl. Cap. vii. 346 (var.) ; Nat. Pl. 119 (var.)

Bulawayo, Rogers, 5880; Salisbury, Rogers, 5798.

A. hirtiflorus, Kunth. var. semiberbis, Stapf.

Fl. Cap. vii. 337; Nat. Pl. 111.

North Melsetter, 4000-6000 ft. April, Swyn. 1607 ; Chirinda, 3500 ft. Jan. Swyn. 1621.

A. hirtus, $L$.

Fl. Cap. vii. 355 ; Nat. Pl. 125.

Matopos, Oct. Gibbs, 288 ; Tabazinduna Mt. Jan. Jeffreys, 45 ; Upper Buzi, 3000-3500 ft. Swyn.; Victoria, Monro, 862,969; Victoria Falls, Rogers, 13278*.

A. intermedius, $R$. $B r$.

Fl. Cap. vii. 345 ; Cat. Afr. Pl. Welw. ii. 149 ; Nat. Pl. 118.

Victoria, Monro, 945a; Victoria Falls, Rogers, 1771*.

A. Jeffreysii, Hack.

Pro. Rhod. Sc. Assn. vii. pt. ii. 70.

Bulawayo, May, Jeffreys, 78.

A. Lecomtei, Franch.

Nyahodi Riv. 4000 ft. April, Swyn. 1660.

A. marginalis, $L$.

Govt. Herb. 634.

A. monticola, Schult. var. Trinii, Hook. (A. Trinii, Steud. var. simplicior, Hack.)

Heaney Jnctn. in vlei, Jeffreys, 26.

A. Nardus, $L$. var. validus, Stapf.

Fl. Cap. vii. 352 ; Nat. Pl. 122.

Salisbury, Mundy.

A. pertusus, Willd.

Fl. Cap. vii. 345 ; Nat. Pl. 466.

Bulawayo, in vlei, Dec. Jeffreys, 57 ; Rogers, 5899; Victoria, Monro, 945a, 983; North Melsetter, 4000-6000 ft. April, Swyn. 1613 ; Victoria Falls, Rogers, 7171.

A. rufus, Kunth. (Cymbopogon rufus, Rendle.)

Fl. Cap. vii. 358 ; Nat. Pl. 127 ; Cat. Afr. Pl. Welw. ii. 155.

Melsetter, 4000-6000 ft. April, Swyn. 1606, 1712 ; Lusitu Riv. 3500 ft. April, Swyn. 1655̃ ; Upper Buzi, 3000-3500 ft. April, Swyn. 993; Victoria Falls, Rogers, 5084 ; Southern Rhodesia, Rand, 411. 
A Record of Plants Collected in Southern Rhodesia.

Genus. No.

134-var. fulyicomus, Rendle.

Upper Buzi, 3000-5000 ft. April, Swyn. 995.

A. Ruprechti, Hack. (Cymbopogon Ruprechti, Rendle.)

Fl. Cap. vii. 365 ; Nat. Pl. 132 ; Cat. Afr. Pl. Welw. ii. 160.

North Melsetter, 4000-5000 ft. April, Swyn. 1605, 1686 ; Nyahodi Riv. 4000 ft. April, Swyn. 1659a; Upper Buzi, 3000-3500 ft. April, Swyn. 991; Matabeleland, Jeffreys, 4, 22 .

A. Schimperi, Hochst. (Cymbopogon Schimperi, Rendle.)

Fl. Cap. vii. 357 ; Nat. Pl. 467 ; Cat. Afr. Pl. Welw. ii. 155.

Salisbury, March, Flanagan, 3000; North Melsetter, 4000-6000 ft. April, Swyn. 951; Upper Buzi, 3000-3500 ft. April, Swyn. $994 a$.

A. schirensis, Huchst.

Fl. Cap. vii. 340 (var.) ; Nat. Pl. 115 (var.); Cat. Afr. Pl. Welw. ii. 148.

Chirinda, 3500 ft. Dec. Swyn. 1624.

A. Schœnanthus, L. (Cymbopogon Schœnanthus, Spreng.)

Fl. Cap. vii. 354 (var.) ; Cat. Afr. Pl. Welw. ii. 154.

Salisbury, Mundy; South Rhodesia, Rand, 410.

var. densiflorus, Hack.

Cat. Afr. Pl. Welw. ii. 154.

Melsetter, 6000 ft. April, Swyn. 2098.

var. versicolor, Hack.

Fl. Cap. vii. 354 ; Nat. Pl. 124.

North Melsetter, 4000-6000 ft. April, Swyn. 1689; Nyahodi Riv. 4000 ft. April, Swyn. 1676; Chirinda, 3500 ft. Jan. Swyn. 1641.

A. serratus, Thunb. var. versicolor, Hack.

Bulawayo, Jeffreys, 5.

A. sorghum, Brot. (Sorghum vulgare, Pers.)

Fl. Cap. vii. 347 ; Nat. Pl. 120; Cat. Afr. Pl. Welw. ii. 150.

A common cultivated crop.

A. squarrosus, L. fil. (A. muricatım, Beauv.).

Cat. Afr. Pl. Welw. ii. 153.

Victoria Falls, Allen, 261.

A. tamba (Rendle), Torre \& Harms. (Cymbopogon tamba, Rendle.) (A.lepidus, Nees, var. tamba, Hack.) (A. cymbarius, L. var. lepidus, Stapf.)

Fl. Cap. vii. 361.

North Melsetter, 4000-6000 ft. April, Swyn. 950, 1690; Chirinda, 3800 ft. April, Swyn. 415 ; Victoria, Monro, 1058. 


\section{A. spp.}

Salisbury, March, Flanagan, 3001.

Govt. Herb. 637.

Craster, 1, 59, 61, 63, 65, 72, 73, 74, 75 .

Syringa, Matabeleland, Govt. Herb. 647.

Somabula, Govt. Herb. 669, nr. Cymbopogon hirtus.

135-Cleistachne sorghoides, Benth.

Mazoe, $4300 \mathrm{ft}$. March, Eyles, 283.

136-Themeda triandra, Forsk. (Anthistiria imberbis, Retz.)

Fl. Cap. vii. 366; Cat. Afr. Pl. Welw. ii. 161.

North Melsetter, 4000-6000 ft. Swyn.; Chirinda, $3500 \mathrm{ft}$. Swyn.; Lusitu Riv. 3000 ft. Swyn.; Nyahodi Riv. 4000 ft. Swyn.

var. glauca, Hack. (T. Forskalii, Hochst. var. glanca, Hack.)

Fl. Cap. vii. see note, p. 368.

Salisbury, Craster, 26 ; Bulawayo, Rogers, 5881, 5903 ; Mashonaland, Govt. Herb. 703.

var. imberbis, Hack.

Fl. Cap. vii. 366 ; Nat. Pl. 133 ; Cat. Afr. Pl. Welw. ii. 161.

Bulawayo, Nov. Jeffreys, 39 ; Salisbury, Darling in Herb. Bolus, 10790 ; Syringa, Matabeleland, Govt. Herb. 631.

\section{T. sp.}

Charter, Govt. Herb. 3012.

143--Tragus racemosus, All.

Fl. Cap. vii. 577 ; Nat. Pl. 404.

Victoria Falls, Allen, 269 ; Bulawayo, in vlei, Dec. Jeffreys, 59 ; Rogers, 5905, 5725 ; Salisbury, Mundy; Charter, Govt. Herb. 3013.

148-Perotis indica, Schum. (P. latifolia, Ait.)

Fl. Cap. vii. 575 ; Nat. Pl. 403 ; Cat. Afr. Pl. Welw. ii. 210 ; Harv. Gen. S.A. Pl. 439.

Victoria Falls, Allen, 262 ; Rogers, 5582 ; Gwai, April, Flanagan, 3221 ; Chirinda', 3700 ft. Swyn.; Salisbury, Craster, 53 ;

Somabula, Govt. Herb. 657; Matabeleland, Jeffreys, 44.

153-Arundinella Ecklonii, Nees.

Fl. Cap. vii. 448 ; Nat. Pl. 183.

Heaney Jnctn. in vlei, Jeffreys, 31; Victoria Falls, Rogers, $5671 *$.

154-Melinis minutiflora, Beauv.

Fl. Cap. vii. 447 ; Nat. Pl. 182 ; Cat. Afr. Pl. Welw. ii. 199.

Chirinda, $3500 \mathrm{ft}$. Swyn. 1694 (? same number given for Pennisetum hordeiforme). 
Fl. Cap. vii. 370 ; Nat. Pl. 134 ; Cat. Afr. Pl. Welw. ii. 162.

Bulawayo, Monro, 991; Victoria Falls, Rogers, 5719; Salisbury, Craster, 17; Mundy; Melsetter, Govt. Herb. 2077 ; Swyn.; Chirinda, $3700 \mathrm{ft}$. Swyn.

P. semialatus (Hook. f.), Torre \& Harms, var. Ecklonii, Stapf. (Axonopus semialatus, Hk. f. var. Ecklonii, Stapf.)

Fl. Cap. vii. 418; Nat. Pi. 166.

Melsetter, $6000 \mathrm{ft}$. Oct. Swyn. 6034 ; Mt. Pene, 6500-7000 ft. Sep. Oct. Swyn. 1644 ; 6035.

\section{P. spp.}

Charter, Govt. Herb. 3014.

Salisbury, Craster, 3.

165-Isachne albens, Trin.

Chipete Forest, 3800 ft. April, Swyn. 406.

166-Panicum bolbodes, Schweinf.

Cat. Afr. Pl. Welw. ii. 170.

North Melsetter, 4000-6000 ft. April, Swyn. 1699.

P. bizanthum, Hochst.

Fl. Cap. vii. 386 ; Nat. Pl. 147 ; Cat. Afr. Pl. Welw. ii. 167.

Salisbury, Darling in Herb. Bolus, 10788; Craster, 29, 35 ; Victoria Falls, Rogers, 5699; North Melsetter, 4000-6000 ft. April, Swyn. 1602a; Nyahodi Riv. 4000 ft. April, Swyn. 1673; Chirinda, 3500 ft. Jan. Swyn. 1622; Enkeldoorn, Govt. Herb. 671.

P. bulawayense, Hack.

Pro. Rhod. Sc. Assn. vii. pt. ii. 69.

Bulawayo, Jeffreys, 28.

P. capillare, $L$.

Fl. Cap. vii. 407 ; Nat. Pl. 498.

Salisbury, Craster, 27 ; Bulawayo, Rogers, 5871*.

P. colonum, $L$.

Cat. Afr. Pl. Welw. ii. 173.

Victoria Falls, Rogers, 5575.

P. coloratum, $L$.

Fl. Cap. vii. 409.

Victoria Falls, Rogers, 5068*.

P commutatum, Nees. (Digitaria eriantha, Steud.)

Fl. Cap. vii. 375 ; Nat. Pl. 137.

Bulawayo, Rogers, 5873 ?; South Rhodesia, Rhod. Agric. Journ. June, 1906, p. 500. 
P. Helopus, Trin.

Fl. Cap. vii. 392, var.; Nat. Pl. 146, var.

Salisbury, Craster, 11; South Rhodesia, Rhod. Agric. Journ. June, 1906, p. 500.

L. heterostachyum, Hack.

Victoria Falls, Rogers, 7034*.

P. Isachne, Roth.

Fl. Cap. vii. 390 ; Nat. Pl. 149.

Matabeleland, Jeffreys, 11 ?; Salisbury, Mundy.

P. lævifolium, Hack.

Fl. Cap. vii. 405 ; Nat. Pl. 157.

Salisbury, Craster, 34 ; Mundy.

P. madagascariense, Spreng.

Cat. Afr. Pl. Welw. ii. 182.

North Melsetter, 3000-6000 ft. April, Swyn. 1702.

P. maximum, Jacq.

Fl. Cap. vii. 404; Nat. Pl. 156 ; Cat. Afr. Pl. Welw. ii. 181.

Heaney Jnctn. by river, Jeffreys, 12 ; Tabainduna Mt. Jeffreys, 32 ; Salisbury, Craster, 67; Mundy; Victoria Falls, Rogers, 7036, 7034, 5630 ; Chirinda, 3700-4000 ft. Swyn.; Bulawayo, Rogers, 5872.

var. trichoglume.

Victoria Falls, Allen, 260.

P. monodactylum, Nees. (Digitaria monodactyla, Stapf.)

Fl. Cap. vii. 373 ; Nat. Pl. 136 ; Cat. Afr. Pl. Welw. ii. 162.

Somabula, Govt. Herb. 663.

P. myosuroides, $R$. Br.

Cat. Afr. Pl. Welw. ii. 174.

Mazoe, 4300 ft. April, Eyles, 320; Victoria Falls, Rogers, 6006 ?

P. nigropedatum, Munro.

Fl. Cap. vii. 388.

Bulawayo, Nov. Jeffreys, 62 ; Rogers, 5901.

P. ramosum, $L$.

Victoria Falls, Rogers, 5720.

P. repens, $L$.

Fl. Cap. vii. 409.

Victoria Falls, Rogers, 5009*. 
Bulawayo, in vlei, Jeffreys, 2 ; South Rhodesia, Rhod. Agric. Journ. 1906, p. 500 ; Victoria Falls, Rogers, 5723*, 5901*.

var. ciliare, Hook. $f$.

Cat. Afr. Pl. Welw. ii. 163.

Victoria Falls, Allen, 271; Chirinda, 3800 ft. April, Swyn. 964.

P. serratum, Spreng.

Fl. Cap. vii. 388 ; Nat. Pl. 148.

Charter, Govt. Herb. 3007 ; Victoria Falls, Rogers, 7057*.

var. holosericeum, Hack.

See Fl. Cap. vii. 389.

Chirinda, 3800 ft. Jan. Swyn. 1623.

P. staginum, Retz.

Fl. Cap. vii. 394 ; Nat. Pl. 492.

Heaney Jnctn. by river, Jeffreys, 3; Salisbury, Mundy, 801 ;

South Rhodesia, Rhod. Agric. Journ. 1906, p. 500.

P. Swynnertonii, Rendle.

Journ. Linn. Soc. Bot. xl. 230.

North Melsetter, 2000-6000 ft. April, Swyn. 1702a.

P. trichopus, Hochst.

Fl. Cap. vii. 391; Nat. Pl. 150.

Victoria Falls, Allen, 267 ; Salisbury, Mundy ; Bulawayo, Rogers, $5891 *$.

P. uncinatum, Raddi.

Cat. Afr. Pl. Welw. ii. 184.

Chipete Forest, 3800 ft. Swyn.; Chirinda, 3700-4000 ft. Swyn.

P. zizanioides, $H . B . K$.

Fl. Cap. vii. 402 ; Nat. Pl. 154.

Salisbury, Craster, 22.

\section{P. spp.}

Victoria Falls, Rogers, 6006, nr. P. hymeniochilum, Nees.

Sebakwe, Govt. Herb. 2078, cf. Digitaria ternata, Stapf.

Matabeleland, Jeffreys, 68, n. sp.? nr. P. serrati, R. Br.

Salisbury, Craster, 9, 19, 20, 23, 24, 81.

167b-Stereochlæna Jeffreysii, Hack.

Pro. Rhod. Sc. Assn. vii. pt. ii. pp. 65, 66.

Bulawayo, May, Jeffreys, 46, 83.

168-Tricholæna glabra, Stapf.

Fl. Cap. vii. 446 ; Nat. Pl. 181.

South Rhodesia, Rhod. Agric. Journ. 1906, p. 500. 
T. rhodesiana, Rendle.

Journ. Linn. Soc. Bot. xl. 232.

Chirinda Forest, 3700-4000 ft. Nov. Swyn. 1632; Nyahodi Riv. 4000 ft. April, Swyn. 1663.

var. glabrescens, Rendle.

Journ. Linn. Soc. Bot. xl. 233.

North Melsetter, 4000-6000 ft. April, Swyn. 1685.

T. rosea, Nees.

Fl. Cap. vii. 443 ; Nat. Pl. 180 ; Cat. Afr. Pl. Welw. ii. 194.

Victoria Falls, Allen, 270; Mazoe, 4400 ft. March, Eyles, 270 ; Bulawayo, Nov. Jeffreys, 53; Rogers, 5516, 5492, 5907, 5874 ; Salisbury, Mundy, 769 ; Craster, 30, 31; Darling, in Herb. Bolus, 10789 ; Rusapi, Engler ; North Melsetter, 4000-6000 ft. Swyn.; Nyahodi Riv. 4000 ft. Swyn.; Chirinda, 3500-3800 ft. Swyn.; Upper Buzi, 3000-3500 ft. Swyn.; Syringa, Matabeleland, Govt. Herb. 653; South Rhodesia, Rand, 409.

169-Oplismenus africanus, Beauv.

Fl. Cap. vii. 416; Nat. Pl. 165 ; Cat. Afr. Pl. Welw. ii. 184.

Chipete Forest, 3800 ft. Swyn.; Victoria Falls, Engler; Rogers, 7400 .

0. sp.

Victoria Falls, Sep. Gibbs, 158.

171-Setaria aurea, $A$. Br.

Fl. Cap. vii. 426 ; Nat. Pl. 479.

Bulawayo, in vlei, Nov. Jeffreys, 37, 56 ; Salisbury, Mundy, 802 ; Craster, 13, 14 ?, 15?; Victoria Falls, Rogers, 7241 ; North Melsetter, 4000-6000 ft. Swyn.; Chirinda, 3000 ft. Swyn.; Upper Buzi, Swyn. ; Charter, Govt. Herb. 3004: Scuth Rhodesia, Rhod. Agric. Journ. 1906, p. 500 ; Somabula, Govt. Herb. 661, 658.

S. flabellata, Stapt.

Fl. Cap. vii. 425.

Salisbury, Mın $\mathbf{n}$ y.

S. Gerrardii, Stapf.

Fl. Cap. vii. 424 ; Nat. Pl. 170.

Bulawayo, Rogers, 5917; Syringa, Matabeleland, Govt. Herb. 632 ; South Rhodesia, Rhod. Agric. Journ. 1906, p. 500.

S. imberbis, Roem. \& Schult.

Fl. Cap. vii. 427 ; Nat. Pl. 173.

Matopos, March, Flanagan, 2996 ; Bulawayo, Rogers, 5879.

S. italica, Beauv.

Fl. Cap. vii. 428.

Bulawayo, Rogers, 5878. 
S. mauritiana, Spreng.

Cat. Afr. Pl. Welw. ii. 187.

Chirinda Forest, 3700-4000 ft. April, Swyn. 417.

S. nigrirostris, Dur. \& Sch.

Fl. Cap. vii. 423 ; Nat. Pl. 169.

North Melsetter, 4000-6000 ft. April, Swyn. 1693; Salisbury, Mundy.

S. verticillata $(L)$, Beanvv.

Fl. Cap. vii. 429 ; Nat. Pl. 174 ; Cat. Afr. Pl. Welw. ii. 185.

Bulawayo, Jeffreys, 6, Rogers, 5996 ; Salisbury, Craster, 38 ; Mundy; Chirinda, 3500 ft. Swyn.; Chepete Forest, $3800 \mathrm{ft}$. Swyn. ; Chipete Forest, 3800 ft. Swyn.

\section{S. spp.}

Charter, Govt. Herb. 3002, 3011.

Salisbury, Craster, 60.

175-Pennisetum cenchroides, Rich. (P. ciliare, Link.)

Fl. Cap. vii. 433 ; Cat. Afr. Pl. Welw. ii. 190.

Tabazinduna Mt. Jan. Jeffreys, 35 ; Bulawayo, Rogers, 5909.

P. macrourum, Trin. (P. hordeiforme, Spreng.)

Fl. Cap. vii. 434.

North Melsetter, 3000-6000 ft. April, Swyn. 1694 (? same number given for Melinis minutiflora); South Rhodesia, Rhod. Agric. Journ. 1906, p. 501.

P. purpureum, Schumach. (P. Benthamii, Steud.)

Kew Bull. 1912, 309 ; Cat. Afr. Pl. Welw. ii. 189.

North Melsetter, 3000-6000 ft. April, Swyn. 955; Lusitu Riv. 3000 ft. Swyn.; Umtali, Engler; Matabeleland, Govt. Herb. 1099.

P. setosum, L. Rich.

Cat. Afr. Pl. Welw. ii. 190.

Upper Buzi, 3000 ft. April, Swyn. 975; Nyahodi Riv. 4000 ft. April, Swyn. 1664.

P. unisetum, Benth. (P. longisetum, K. Schum.) (P. dioicum, A. Rich.)

Fl. Cap. vii. 437 ; Nat. Pl. 178; Cat. Afr. Pl. Welw. ii. 192.

Lusitu Riv. 3000 ft. April, Swyn. 1649; Upper Buzi, 30003500 ft. April, Swyn. 983 ; Chirinda, 3500 ft. Swyn. 414.

184-Olyra latifolia, $L$.

Fl. Cap. vii. 746 ; Nat. Pl. 464 ; Cat. Afr. Pl. Welw. ii. 255.

Chipete Forest, 3800 ft. Swyn.

187-Leptaspis cochleata, Thw.

Cat. Afr. Pl. Welw. ii. 256.

Chirinda, 3700-4000 ft. Dec. and Jan. Swyn. 410, 419, 6633. 
Genus No.

208-Äristida adscensionis, $L$.

Fl. Cap. vii. 554 ; Cat. Afr. Pl. Welw. ii. 202.

North Melsetter, 4000 ft. Swyn.; Syringa, Govt. Herb. 639 ;

Bulawayo, Rogers, 5968 ; Victoria Falls, Rogers, 5863.

A. æquiglumis, Hack.

Fl. Cap. vii. 555 ; Nat. Pl. 197.

South Rhodesia, Rhod. Agric. Journ. 1906, p. 500.

A. angustata, $\operatorname{Stapf}$.

Fl. Cap. vii. 556 ; Nat. Pl. 198.

Heaney Jnctn. Jeffreys, 10 ; Bulawayo, Rogers, 13510*.

A. barbicollis, Trin. \& Rupr.

Fl. Cap. vii. 559 ; Nat. Pl. 401.

North Melsetter, 4000 ft. April, Swyn. 1717 ; Salisbury, Craster, 50 ; Bulawayo, Rogers, 5904, 5887.

A. bipartita, Trin. \& Rupr.

Fl. Cap. vii. 558 ; Nat. Pl. 483.

Matabeleland, Jeffreys, 63 ; Victoria Falls, Rogers, 7040*.

A. ciliata, Desf.

Fl. Cap. vii. 563.

South Rhodesia, Rhod. Agric. Journ. 1906, p. 500.

A. congesta, Roem. \& Schult.

Fl. Cap. vii. 558 ; Nat. Pl. 484.

Matabeleland, Jeffreys, 87.

A. stipitata, Hack. (A. sieberiana, Trin. var. stipitata, Stapf.)

Fl. Cap. vii. 560.

Matabeleland, Jeffi eys, 23.

A. stipoides, Lam.

Fl. Cap. vii. 562.

Matopos, Engler; North of Bulawayo, Engler.

\section{A. spp.}

Victoria Falls, Allen, 263.

Ingesi, Matabeleland, Govt. Herb. 679.

Charter, Govt. Herb. 3001, 3010.

230-Sporobolus centrifugus, Nees.

Fl. Cap. vii. 584 ; Nat. Pl. 406.

Mt. Pene, 6500-7000 ft. Sep. Swyn. 1647.

S. festivus, Hochst.

Cat. Afr. Pl. Welw. ii. 207.

Victoria Falls, Rogers, 5713 ; Syringa, Govt. Herb. 643 ; Salisbury, Craster, 8 .

var. fibrosus.

Victoria Falls, Allen, 268. 
Genus No.

var. stuppeus, Stapf.

Fl. Cap. vii. 582 ; Nat. Pl. 405 ; in Pro. Rhod. Sc. Assn. vii.pt. ii. p. 64, Hackel says, "A distinct species according to my opinion."

Heaney Jnctn. in vlei, Jan. Jeffreys, 34.

S. fimbriatus, Nees.

Fl. Cap. vii. 585 ; Nat. Pl. 486.

Victoria, Monro, 992 ; Salisbury, Rogers, 13509.

S. indicus, $R . B r$.

Fl. Cap. vii. 586 ; Nat. Pl. 408.

Salisbury, Darling, in Herb. Bolus, 10791 ; Mundy; Chirinda, 3700 ft. Jan. Swyn. 404.

var. laxus, Stapf. (S. pyramidalis, Nees.)

Fl. Cap. vii. 586.

Heaney Jnctn. Jeffreys, 24 ; Bulawayo, Rogers, 5877.

S. panicoides, Rich.

Bulawayo, Rogers, 5864.

S. spp.

Salisbury, Craster, 28, 33, 44, 80.

277-Tristachya biseriata, Stapf.

Fl. Cap. vii. 453 ; Cat. Afr. Pl. Welw. ii. 217.

North Melsetter, 3000-6000 ft. April, Swyn. 1708.

T. leucothrix, Trin.

Fl. Cap. vii. 453 ; Nat. Pl. 187.

Mt. Pene, 6500-7000 ft. Sep. Swyn. 1643.

278-Trichopteryx gigantea, Stapf.

South of Victoria Falls, Baines ; Holub.

T. simplex, Hack.

Fl. Cap. vii. 450 ; Nat. Pl. 185.

North Melsetter, 4000-6000 ft. fr. April, Swyn. 1612, 1709 ;

Nyahodi Riv. 4000 ft. April, Swyn. 1662 ; Chipetzana Source, 4000 ft. fr. April, Swyn. 974 ; Chirinda, 3700 ft. Jan. Swyn.

1618; Salisbury, Govt. Herb. 604 ; Matabeleland, Jeffreys, 15.

var. minor, Stapf.

Fl. Cap. vii. 450.

Salisbury, Craster, 21 ; Syringa, Govt. Herb. 649.

\section{T. spp.}

Salisbury, Craster, 55.

Engler, probably T. simplex, Hack.

Matapos, Rogers, 5194, probably n. sp.

280-Danthonia inermis, Stapf. (Pentaschistis inermis.)

Fl. Cap. vii. 534.

Victoria Falls, Rogers, 5671. 
Genus No.

281-Microchloa caffra, Nees.

Fl. Cap. vii. 636 ; Nat. Pl. 431; Harv. Gen. S.A. Pl. 447.

Matabeleland, Jeffreys, 58.

282-Cynodon Dactylon, Pers.

Fl. Cap. vii. 634 ; Nat. Pl. 430 ; Cat. Afr. Pl. Welw. ii. 220.

Victoria Falls, Allen, 276; Rogers, 5718, 5876; Bulawayo, Dec. Jeffreys, 61 ; Rogers, 5514 ; Chubb, 92 ; Salisbury, Craster, 10 ; Mundy; Chirinda, 3800 ft. Swyn.

C. incompletus, Nees.

Fl. Cap. vii. 635.

Salisbury, Mundy.

286-Ctenium sp.

Salisbury, Mundy.

288--Chloris barbata, $S w$.

Cat. Afr. Pl. Welw. ii. 222.

Chirinda, 3500 ft. Swyn. ; North Melsetter, 4000 ft. Swyn.

C. gayana, Kunth. (C. abyssinica, Hochst.)

Fl. Cap. vii. 642 ; Nat. Pl. 437.

North Melsetter, 4000-6000 ft. April, Swyn. 1700; Salisbury, Mundy.

C. petræa, Thunb.

Fl. Cap. vii. 643 ; Nat. Pl. 438.

Matabeleland, Jeffreys, 52.

C. pycnothrix, Trin.

Fl. Cap. vii. 641 ; Nat. Pl. 435.

Victoria Falls, April, Flanagan, 3224 ; Chirinda, 3700 ft. Oct. and April, Swyn. 1629a, 1696; Salisbury, Craster, 25.

C. virgata, Swartz.

Fl. Cap. vii. 641 ; Nat Pl. 436.

Salisbury, Mundy, 768; Bulawayo, Rogers, 5875 ; Matabeleland, Jeffreys, 86; South Rhodesia, Rhod. Agric. Journ. 1906, p. 500 .

var. elegans, Stapf.

Fl. Cap. vii. 642.

Salisbury, Craster, 7.

294-Craspedorhachis rhodesiana, Rendle.

Journ. Linn. Soc. Bot. xl. 233.

North Melsetter, 3000-6000 ft. April, Swyn. 1697.

C. sp.

Salisbury, Craster, 79.

298-Tripogon abyssinicus, Nees.

Cat. Afr. Pl. Welw. ii. 223.

Matabeleland, Jeffreys, 41. 
A Record of Plants Collected in Southern Rhodesia.

Genus No.

304-Eleusine coracana, Gaertn.

Fl. Cap. vii. 645; Nat. Pl. 440 ; Cat. Afr. Pl. Welw. ii. 224.

South Rhodesia, Rhod. Agric. Journ. 1905, p. 115. A common native crop.

E. indica, Gaertn.

Fl. Cap. vii. 645 ; Nat. Pl. 439 ; Cat. Afr. Pl. Welw. ii. 224.

Heaney Jnctn. Jeffreys, 29; Salisbury, Mundy, 738; Craster, 36; North Melsetter, 6000 ft. Swyn.; Chirinda, $3500 \mathrm{ft}$. Swyn.

305-Dactyloctenium ægyptiacum, Willd.

Fl. Cap. vii. 646 ; Nat. Pl. 441 ; Cat. Afr. Pl. Welw. ii. 224.

Heaney Jnctn. Jeffreys, 30 ; Victoria Falls, Rogers, 5592, 5726 ; North Melsetter, 4000-6000 ft. Swyn.; Bulawayo, Rogers, 5886.

$309 b$-Crossotropis grandiglumis, Rendle.

Fl. Cap. vii. 649 ; Nat. Pl. 443 ; Cat. Afr. Pl. Welw. ii. 226.

Heaney Jnctn. Jeffreys, 1 ; Bulawayo, Rogers, 5883*.

310-Pappophorum molle, Kunth. (Enneapogon mollis, Lehm.)

Fl. Cap. vii. 655.

Victoria Falls, 5694.

312-Schmidtia bulbosa, Stapf.

Fl. Cap. vii. 658.

Victoria Falls, Rogers, 5724 ; Bulawayo, Rogers.

S. pappophoroides, Steud. (Antoschmidtia pappophoroides, Boiss.)

Cat. Afr. Pl. Welw. ii. 231; also see note under S. bulbosa, Stapf, Fl. Cap. vii. 658.

Victoria Falls, Allen, 273.

333-Phragmites communis, Trin. (Trichoon Phragmites, Rendle.)

Fl. Cap. vii. 541; Nat. Pl. 193 ; Cat. Afr. Pl. Welw. ii. 218.

Nyahodi Riv. 4000 ft. Swyn. ; Lusitu Riv. 2500 ft. Swyn. ; Salis-

bury, Craster, 68; Matopos, Rogers, 5196 (forma); Victoria

Falls, Engler ; Umtali, Engler.

P. sp.

Victoria Falls, Rogers, 5317.

337-Diplachne alopecuroides (Stapf), Torre \& Harms. (Leptocarydion alopecuroides, Stapf.)

Fl. Cap. vii. 649 ; Cat. Afr. Pl. Welw. ii. 225.

North Melsetter, 3000-6000 ft. April, Swyn. 1695 ; Victoria Falls, Rogers, 7029*.

D. biflora, Hack. var. Buchananii, Stapf.

Fl. Cap. vii. 593 ; Nat. Pl. 411.

Chirinda, 3500 ft. Jan. Swyn. 1619; Syringa, Govt. Herb. 651. 
Genus No.

337 -Pogonarthria Marlothii, Hack. (Eragrostis Marlothii, Hack,; Leptochloa falcata, Hack.; Pogonarthria falcata, Rendle.) See note by Hackel in Pro. Rhod. Sc. Assn. vii. pt. ii. 64 . Fl. Cap. vii. 589 ; Nat. Pl. 409 ; Cat. Afr. Pl. Welw. ii. 232. Victoria Falls, Rogers, 6013, 7043 ; North Melsetter, 3000-6000 ft. April, Swyn. 1609, 1706 ; Nyahodi Riv. 4000 ft. April, Swyn. 1674a ; Upper Buzi, 3000-3500 ft. April, Swyn. 978, 981, 982 ; Salisbury, Craster, 52, 57, 71; North of Bulawayo, Engler; Matabeleland, Jeffreys, 27, 79.

\section{1-Eragrostis abyssinica?.}

Govt. Herb. 2079.

E. aspera, Nees.

Fl. Cap. vii. 628 ; Nat. Pl. 424 ; Cat. Afr. Pl. Welw. ii. 232.

Chirinda, 3700-4000 ft. Swyn.; Victoria Falls, Rogers, 7038*.

E. Atherstonei, Stapf.

Fl. Cap. vii. 607 ; Nat. Pl. 426.

Somabula, Govt. Herb. 667 ; Matabeleland, Jeffreys, 19.

E. barbinodis, Hack.

Fl. Cap. vii. 621.

Bulawayo, March, Jeffreys, 51.

E. brizoides, Nees.

Fl. Cap. vii. 622 ; Nat. Pl. 422 ; Cat. Afr. Pl. Welw. ii. 251.

Mt. Pene, 6500 ft. Sep. Swyn. 1646 ; Charter, Govt. Herb. 3009 ; Salisbury, Mundy; Victoria Falls, Rogers; South Rhodesia, Rhod. Agric. Journ. 1906, p. 501.

E. chalcantha, Trin.

Fl. Cap. vii. 615 ; Nat. Pl. 418 ; Cat. Afr. Pl. Welw. ii. 248.

Victoria Falls, Allen, 274 ; Heaney Jnctn. by river, Jeffreys, 18 ; North Melsetter, 3000-6000 ft. Swyn.; Enkeldoorn, Govt. Herb. 672 ; Somabula, Govt. Herb. 662 ; Umtali, Engler.

E. Chapelieri, Nees.

Fl. Cap. vii. 614 ; Nat. Pl. 417 ; Cat. Afr. Pl. Welw. ii. 250.

North Melsetter, 3000-6000 ft. Swyn. 1707; Nyahodi Riv. 4000 ft. Swyn. 1674; Upper Buzi, 3000-3500 ft. April, Swyn. 980 ; Umtali, Engler; Matabeleland, Jeffreys, 20.

E. chloromelas, Steud.

Fl. Cap. vii. 602.

Bulawayo, Rogers, 5892.

E. congesta, Oliv.

Nyahodi Riv. 4000 ft. April, Swyn. 1665.

E. curvula, Nees.

Fl. Cap. vii. 599 ; Nat. Pl. 413 ; Cat. Afr. Pl. Welw. ii. 244.

Bulawayo, Rogers, 5515; Salisbury, Mundy. 
Fl. Cap. vii. 613.

Syringa, Govt. Herb. 650 ; Victoria Falls, Rogers, 5721*.

E. gangetica, Steud.

Fl. Cap. vii. 617 ; Nat. Pl. 419.

Victoria Falls, Rogers, 7044 ; Matopos, Rogers, 5694.

E. gummiflua, Nees.

Fl. Cap. vii. 629 ; Nat. Pl. 425.

Heaney Jnctn. in vlei, Jeffreys, 13; Bulawayo, Rogers, 5884 ;

Salisbury, Mundy.

E. hispida, K. Schum.

Salisbury, Craster, 16.

E. Jeffreysii, Hack.

Pro. Rhod. Sc. Assn. vii. pt. ii. 68.

Bulawayo, March, Jeffreys, 42.

E. Lappula, Nees, var. divaricata, Stapf, forma macra, Hack.

Fl. Cap. vii. 628.

Matabeleland, Jeffreys, 75.

E. lehmanniana, Nees.

Fl. Cap. vii. 601.

Bulawayo, Rogers, 5895 ?; Somabula, Govt. Herb. 659.

E. major, Host.

Fl. Cap. vii. 620 ; Nat. Pl. 420 ; Cat. Afr. Pl. Welw. ii. 237.

North Melsetter, 4000-6000 ft. Swyn. ; Upper Buzi, 3000-3500 ft.

Swyn.; between Wankie and Victoria Falls, Rogers, 6005 ;

Bulawayo, Rogers, 5869, 5867; Victoria Falls, Rogers, 5787 ?

E. namaquensis, Nees. (E. interrupta, Beauv. var. namaquensis, Dur. \& Schinz.)

Fl. Cap. vii. 630 ; Cat. Afr. Pl. Welw. ii. 233.

North Melsetter, 4000-6000 ft. April, Swyn. 1608.

var. robusta, Stapf.

Fl. Cap. vii. 630 ; Nat. Pl. 429.

Matabeleland, Jeffreys, 25.

E. patentipilosa, Hack.

Pro. Rhod. Sc. Assn. vii. pt. ii. 67.

Bulawayo, May, Jeffreys, 33.

E. plana, Nees.

Fl. Cap. vii. 609 ; Nat. Pl. 416 ; Cat. Afr. Pl. Welw. ii. 251.

Melsetter, $6000 \mathrm{ft}$. April, Swyn. 1681; Chirinda, 3500 ft. Nov.Jan. Swyn. 404a, 968, 1626.

E. plumosa, $\operatorname{Link}$.

Matopos, Rogers, 5405 ; Bulawayo, Rogers, 5888. 
Genus No.

E. sclerantha, Nees, var. retinorrhœa, Steud.

Fl. Cap. vii. 615, type.

Matopos, Engler.

E. superba, Peyr.

Fl. Cap. vii. 622 ; Nat. Pl. 421 ; Cat. Afr. Pl. Welw. ii. 251.

Bulawayo, in vlei, Dec. Jeffreys, 38, 50 ; Rogers, 5908; Charter, Govt. Herb. 3003 ; Salisbury, Mundy.

E. tenella, Stapf, var. plumosa.

Matopos, March, Flanagan, 2943.

E. uniglumis, Hack.

Pro. Rhod. Sc. Assn. vii. pt. ii. 66.

Heaney Jnctn. Jan. Jeffreys, 48 ; Salisbury, Mundy.

E. viscosa, Trin. (E. tenella, Beauv. var. viscosa, Stapf.)

Cat. Afr. Pl. Welw. ii. 233.

Heaney Jnctn., in vlei, Jeffreys, 14 ; Salisbury, Mundy.

E. Wilmsii, $\operatorname{Stapf}$.

Fl. Cap. vii. 606.

Syringa, Govt. Herb. 640.

E. spp.

Victoria Falls, April, Flanagan, 3212, 3222.

Victoria Falls, Allen, 266, cf. E. pilosa, Beauv.

Victoria Falls, Rogers, 5418.

Syringa, Govt. Herb. 638, 644, 652, 655, 660, 682.

Charter, Govt. Herb. 3005, 3008.

Salisbury, Craster, 4, 5, 6, 32, 39, 54, 58, 64, 76.

346-Kœ leria cristata, Pers.

Fl. Cap. vii. 468 ; Nat. Pl. 189.

Mt. Pene, 6500-7000 ft. Swyn.

361-Pœ cilostachys flaccidula, Stapf.

Journ. Linn. Soc. Bot. xl. 231.

Chipete Forest, $3800 \mathrm{ft}$. April, Swyn. 409a.

385-Festuca sp.

Bulawayo, 4500 ft. Dec. Eyles, 1203, cf. F. costata, Nees.

395-Lolium sp.

Victoria, Munro, 492.

405-Agropyrum lepidus, ? var. tamba.

Victoria, Munro, 1058.

430-0xytenanthera abyssinica, Munro .

Cat. Afr. Pl. Welw. ii. 256.

Victoria Falls, Rogers, 5603. 
FAMILY XX.-CYPERACEAE, J. St. Hil.

Genus No

452-Lipocarpha argentea, $R$. Br.

Fl. Cap. vii. 265 ; Fl. Trop. Afr. viii. 469 ; Cat. Afr. Pl. Welw. ii. 129.

Matopos, Rogers, 5262 ; Chirinda, $3500 \mathrm{ft}$. Swyn.

L. monocephala, Turrill.

Kew Bull. 1913, 307.

Victoria Falls, Rain Forest, Rogers, 6024.

454-Ascolepis capensis, (Kunth.), Ridley.

Fl. Cap. vii. 266; Fl. Trop. Afr. viii. 477 ; Cat. Afr. Pl. Welw. ii. 131.

Marandellas, Engler ; Rogers, 4040; South. Rhodesia, Bryce.

A. protea, Welw.

Fl. Trop. Afr. viii. 474 ; Cat. Afr. Pl. Welw. ii. 130.

Victoria Falls, Allen, 22.

var. bellidiflora, Welw.

Fl. Trop. Afr. viii. 475; Cat. Afr. Pl. Welw. ii. 130.

Victoria, Monro, 905.

459-Cyperus albostriatus, Schrad.

Fl. Cap. vii. 176.

Mt. Pene, 7000 ft. Oct. Swyn. 6036.

C. amabilis, Vahl.

Fl. Trop. Afr. viii. 327; Cat. Afr. Pl. Welw. ii. 109.

Salisbury, Craster, 47 ; Victoria Falls, Rogers, 601.1.

C. aristatus, Rottb.

Fl. Trop. Afr. viii. 348; Fl. Cap. vii. 179; Cat. Afr. Pl. Welw. ii. 110.

Victoria Falls, Allen, 286 ; Bulawayo, Rogers, 5816.

C. auricomus, $S p r$. ?

Bulawayo, Rogers, 5512.

C. betschuanus, Boeck. (Pycreus betschuanus, C. B. Cl.)

Fl. Trop. Afr. viii. 304; Fl. Cap. vii. 159.

Mashonaland, Bryce.

C. compactus, Lam.

Fl. Trop. Afr. viii. 319 ; Fl. Cap. vii. 168; Cat. Afr. Pl. Welw. ii. 112.

North Melsetter, 2000-6000 ft. April, Swyn. 916b; Mt. Pene, 6800 ft. Sep. Swyn. 916; Somabula, Govt. Herb. 665; Salisbury, Rogers, 4087; Matabeleland, Elliott.

var. flaxissimus, $C . B . C l$.

Fl. Trop. Afr. viii. 320 ; Cat. Afr. Pl. Welw. ii. 112.

Victoria, Monro, 676. 
Fl. Trop. Afr. viii. 338 ; Fl. Cap. vii. 173 ; Cat. Afr. Pl. Welw. ii. 114

Matopos, fl. \& fr. Sep. Gibbs, 56 ; Victoria Falls, on island, Kirk ; Rogers, 5966.

C. distans, L. fil.

Fl. Trop. Afr. viii. 349 ; Fl. Cap. vii. 178; Cat. Afr. Pl. Welw. ii. 116.

Chirinda, 3700 ft. Swyn.; Victoria Falls, Rogers, 5600 ; Bulawayo, Rogers, 5813.

C. esculentus, $L$.

Fl. Trop. Afr. viii. 355 ; Fl. Cap. vii. 180 ; Cat. Afr. Pl. Welw. ii. 117.

Gwai, April, Flanagan, 3215 ?; Salisbury, Craster, 40, 41.

C. globosus, Boeck. (Pycreus globosus, Reichb. var. nilagirica, C. B. Cl.)

Fl. Trop. Afr. viii. 299 ; Fl. Cap. vii. 159.

Umtali, Engler.

C. Haspan, $L$.

Fl. Trop. Afr. viii. 332 ; Cat. Afr. Pl. Welw. ii. 114.

Victoria Falls, Engler; Rogers, 5081 ; Chirinda, 3500 ft. Swyn. var. americana, Boeck.

Victoria Falls, on bog edge, fl. \& fr. Sep. Gibbs, 167.

C. hemisphæricus (C.B.Cl.), Torre \& Harms. (Mariscus hemispharicus, C. B. Cl.)

Fl. Trop. Afr. viii. 400.

Chirinda, 3800 ft. April, Swyn. 412.

C. margaritaceus, Vahl.

Fl. Trop. Afr. viii. 321 ; Fl. Cap. vii. 169 ; Cat. Afr. Pl. Welw. ii. 112 .

Gwai, April, Flanagan, 3216 ; Salisbury, Craster, 45; Rogers, 4087.

C. Mundtii, Kunth. (Pycreus Mundtii, Nees.)

Fl. Trop. Afr. viii. 294; Fl. Cap. vii. 157; Cat. Afr. Pl. Welw. ii. 106.

Victoria Falls, in bog, fl. \& fr. Sep. Gibbs, 149 ; Engler.

C. papyrus, $L$.

Fl. Trop. Afr. viii. 374 ; Cat. Afr. Pl. Welw. ii. 118.

Victoria Falls, on island, Rogers, 5103 ; Matopos, Rogers.

C. polystachyus, Rottb. (Pycreus polystachyus, Beauv.)

Fl. Trop. Afr. viii. 296 ; Fl. Cap. vii. 157 ; Cat. Afr. Pl. Welw. ii. 108.

Sabi Riv. 1000 ft. Swyn. 
Genus No.

var. laxiflora, Benth.

Fl. Trop. Afr. viii. 297; Fl. Cap. vii. 158.

Chirinda, $3500 \mathrm{ft}$. Swyn.

C. pulcher, Thunb. (C. dregeamus, Kunth.)

Fl. Cap. vii. 171.

Victoria Falls, Rogers, 5619.

C. radiatus, Vahl. (Mariscus radiatus?)

Fl. Trop. Afr. viii. 369.

Victoria Falls, Rogers, 5574.

C. sieberianus (Nees), Torre \& Harms. (Mariscus sieberianus, Nees.)

Fl. Trop. Afr. viii. 388 ; Fl. Cap. vii. 189.

Chirinda, $3800 \mathrm{ft}$. Swyn.

C. sphærospermus, Schrad.

Fl. Trop. Afr. viii. 336 ; Fl. Cap. vii. 172 ; Cat. Afr. Pl. Welw. ii. 115 .

Salisbury, Craster, 48 ?

C. textilis, Thunb.

Fl. Cap. vii. 174.

Mazoe, 4300 ft. Sep. Eyles, 425 ; Bulawayo, Chubb, 30.

C. Zollingeri, Steud.

Fl. Trop. Afr. viii. 360 ; Cat. Afr. Pl. Welw. ii. 117.

Victoria Falls, Allen, 279.

C. spp.

Victoria Falls, April, Flanagan, 3213, cf. C. nitens, Retz.

Victoria Falls, April, Flanagan, 3214, cf. C. aureobruneus, C. B. Cl.

Gwai, April, Flanagan, 3220, cf. C.amabilis, Vahl. and 3217.

Matopos, March, Flanagan, 2983.

Victoria, Monro, 1057.

Bulawayo, Nov. Eyles, 1135, 1187.

South Rhodesia, Rand, 255, 256, 257, 258, 259, 406, 407.

461-Courtoisia assimilis, C. B. Cl.

Fl. Trop. Afr. viii. 404.

Matopos, Rogers, 5403.

C. cyperoides, Nees.

Fl. Trop. Afr. viii. 404.

Matabeleland, Holub.

462-Kyllinga alba, Nees.

Fl. Trop. Afr. viii. 271; Fl. Cap. vii. 151; Cat. Afr. Pl. Welw. ii. 105,

Chirinda, 3800 ft. May, Swyn. 459; Bulawayo, Chubb, 384 ; banks of Zambesi, Holub.

K. erecta, Schum.

Fl. Cap. vii. 152 ; Cat. Afr. Pl. Welw. ii. 105.

Bulawayo, Rogers, 5513. 


\section{K. spp.}

Salisbury, Darling, in Herb. Bolus, 2 spp.

- Victoria Falls, Allen, 80.

467-Fuirena glomerata, Lam.

Fl. Trop. Afr. viii. 465 ; Cat. Afr. Pl. Welw. ii. 128.

Ngomi, April, Flanagan, 3218.

F. hirta, Vahl.

Fl. Cap. vii. 264.

Victoria Falls, Engler.

F. Oedipus, C. B. Cl.

Journ. Linn. Soc. Bot. xxxvii. 478.

Victoria Falls, Rain Forest, Sep. Gibbs, 125.

F. pachyrrhiza, Ridl.

Fl. Trop. Afr. viii. 464 ; Fl. Cap. vii. 262 ; Cat. Afr. Pl. Welw. ii. 129 .

Salisbury, Craster, 49.

F. pubescens, Kunth.

Fl. Trop. Afr. viii. 463; Fl. Cap. vii. 261; Cat. Afr. Pl. Welw. ii. 128 .

Chirinda, 3500 ft. Swyn.; Macheke, Rogers, 4050 ; Bulawayo, Rogers, 5517.

F. stricta, Steud.

Fl. Trop. Afr. viii. 465.

Matopos, fl. \& fr. Sep. Gibbs, 92; Chirinda, 3700 ft. Jan. Swyn. 910.

F. subdigitata, C. B. Cl.

Journ. Linn. Soc. Bot. xxxvii. 477.

Matopos, Sep. Gibbs, 196.

F. umbellata, Rottb.

Fl. Trop. Afr. viii. 466.

Victoria Falls, Rogers, 5093*.

F. welwitschii, Ridley.

Fl. Trop. Afr. viii. 463.

Victoria Falls, Rogers, 13600*.

\section{F. spp.}

Bulawayo, Dec. Eyles, 1136.

Matopos, March, Flanagan, 2976.

Matopos, Sep. Gibbs, 54.

468-Scirpus articulatus, $L$.

Fl. Trop. Afr. viii. 453 ; Fl. Cap. vii. 228 ; Cat. Afr. Pl. Welw. ii. 126.

Victoria, Monro, 912. 
A Record of Plants Collected in Southern Rhodesia.

Genus No.

S. corymbosus, Roth.

Fl. Trop. Afr. viii. 455 ; Fl. Cap. vii. 229 ; Cat. Afr. Pl. Welw. ii. 126.

Melsetter, $6000 \mathrm{ft}$. Sep. Swyn. 906; Inyamadzi Valley, $3000 \mathrm{ft}$. Oct. Swyn. 905.

S. fluitans, $L$.

Fl. Trop. Afr. viii. 449 ; Fl. Cap. vii. 213 ; Cat. Afr. Pl. Welw. ii. 126.

Matopos, in streams, fl. \& fr. Sep. Gibbs, 211.

S. paludicola, Kunth .

Fl. Cap. vii. 230.

Victoria Falls, fl. \& fr. Sep. Gibbs, 168 ; Engler.

S. supinus, $L$.

Fl. Trop. Afr. viii. 452.

Matopos, fl. \& fr. Sep. Gibbs, 58.

469-Heleocharis capitata, Boeck. (Eleocharis capitata, R. Br.)

Fl. Trop. Afr. viii. 407.

Victoria Falls, Rain Forest, fl. \& fr. Sep. Gibbs, 162 ; Engler.

H. Kirkii (C. B. Cl.), Torre \& Harms. (Eleocharis Kirkii, C. B. Cl.)

Fl. Trop. Afr. viii. 410.

Victoria Falls, on island, Kirk.

471-Fimbristylis Burchellii, Fic. \& Hiern. (Bulbostylis Burchelli, C. B. Cl.)

Fl. Trop. Afr. viii. 440 ; Fl. Cap. vii. 210 ; Cat. Afr. Pl. Welw. ii. 125 .

Mazoe, Dec. Eyles, 483; Victoria Falls, April, Flanagan, 3223 ; Rogers, 7269; Salisbury, Craster, 42, 46; Bulawayo, Rogers, 5815 ; Ingesi, Govt. Herb. 675.

F. diphylla, Vahl.

Fl. Trop. Afr. viii. 415 ; Fl. Cap. vii. 200 ; Cat. Afr. Pl. Welw. ii. 122 .

Chirinda, 3700 ft. Swyn.; Salisbury, Craster, 43.

F. exilis, Roem. \& Schl.

Fl. Trop. Afr. viii. 418; Fl. Cap. vii. 201 ; Cat. Afr. Pl. Welw. ii. 123.

Victoria Falls, Allen, 265; Matopos, Rogers, 5170; Chirinda, 3000-6000 ft. Swyn.; Chimanimani Mts. 7000 ft. Swyn.; Upper Buzi, Swyn.

F. rhodesiana, Rendle.

Journ. Linn. Soc. Bot. xl. 222.

Upper Buzi, 4000-3500 ft. April, Swyn. 920. 
F. schœnoides (Kunth.). Torre \& Harms. (Bulbostylis schœnoides, Kunth.)

Fl. Cap. vii. 206.

Marandellas, Rogers, 4035.

F. spp.

Matopos, Sep. Galpin, 7072 ; March, Flanagan, 2984.

Mazoe, Dec. Eyles, 482.

512-Eriospora villosula, C. B. Cl.

Fl. Trop. Afr. viii. 513.

Chimanimani Mts. 7000 ft. Sep. Swyn. 918.

515-Scleria Buchanani, Boeck.

Fl. Trop. Afr. viii. 499 ; Fl. Cap. vii. 295.

Salisbury, Craster, 12 ; Victoria, Monro, 633.

S. foliosa, A. Rich.

Fl. Trop. Afr. viii. 503.

Victoria Falls, Rogers, 7169.

S. sp.

Matopos, 5000 ft. Nov. Eyles, 1107.

525-Carex condensata, Nees.

Fl. Trop. Afr. viii. 521 ; Fl. Cap. vii. 305.

Mt. Pene, 6500-7000 ft. Sep. Swyn. 903.

C. spicato-paniculata, C. B.Cl.

Fl. Trop. Afr. viii. 520; Fl. Cap. vii. 304.

Chirinda, 3000 ft. June, Swyn. 413.

\section{Series PRINCIPES.}

FAMILY XXI.-PALMAE, Linn.

528-Phoenix dactylifera, $L$.

Fl. Trop. Afr. viii. 102 ; For. Fl. Port. E. Afr. 110.

Chirinda, 3500 ft. fl. \& fr. Oct. Swyn. 666.

P. reclinata, Jacq.

For. Fl. Cape, 341 ; Cat. Afr. Pl. Welw. ii. 82 ; Fl. Cap. vii. 29 ;

Fl. Trop. Afr. viii. 103 ; For. Fl. Port. E. Afr. 110.

Victoria Falls, Davy; Palm Grove, Engler.

553-Hyphæne crinita, Gaertn.

Fl. Trop. Afr. viii. 121 ; Fl. Cap. vii. 30 ; For. Fl. Port. E. Afr. 111.

Gwanda, Noble, 77.

H. ventricosa, Kirk.

Fl. Trop. Afr. viii. 122.

Victoria Falls, Kirk; Engler. 


\section{SERIES SPATHIFLORAE. \\ FAMILY XXIII.-ARACEAE, Neck.}

Genus No.

690-Culcasia scandens, Beauv.

Fl. Trop. Afr. viii. 174 ; Cat. Afr. Pl. Welw. ii. 90.

Chirinda Forest, 3700-4000 ft. fl. \& fr. Jan. Swyn. 98.

723--Amorphophallus mossambicensis, Klotzsch.

Fl. Trop. Afr. viii. 150.

Victoria Falls, Allen, 42.

\section{A. sp.}

Mazoe, Mundy.

748-Zantedeschia melanoleuca, Engl. var. tropicalis, N. E. Br. (Richardia melanoleuca, Hook. f.)

Fl. Trop. Afr. viii. 168 ; Fl. Cap. vii. 38.

Salisbury, Cecil, 149.

\section{SERIES FARINOSAE.}

FAmily XXIX.-XYRIDACEAE, Lindl.

826-Xyris capensis, Thunb.

Fl. Trop. Afr. viii. 13; Fl. Cap. vii. 6; Cat. Afr. Pl. Welw. ii. 68.

Matopos, 4800 ft. March, Eyles, 1031; Sep. Gibbs, 85.

X. dispar, N. E. Br.

Fl. Trop. Afr. viii. 12.

Salisbury, Cecil, 152.

X. Hildebrandtii, Nilss. (X. Umbilonis, Nilss.)

Fl. Trop. Afr. viii. 24 ; Fl. Cap. vii. 4 ; Cat. Afr. Pl. Welw. ii. 67.

Matopos, $4800 \mathrm{ft}$. March, Eyles, 1029.

X. multicaulis, N. E. Br.

Fl. Trop. Afr. viii. 20.

Victoria Falls, Rain Forest, Galpin, 7040; Sep. Gibbs, 169 ;

Engler.

X. obscura, N. E. Br.

Fl. Trop. Afr. viii. 16.

Salisbury, Cecil, $152 a$.

\section{X. spp.}

Matopos, March, Flanagan, 2970.

South Rhodesia, Rand, 248. 
Family XXX.-ERIOCAULACEAE, Lindl.

Genus No.

828-Eriocaulon africanum, Hochst.

Fl. Cap. vii. 56.

Odzani River Valley, Umtali; Teague, 239.

E. amphibium, Rendle.

Journ. Linn. Soc. Bot. xxxvii. 475.

Matopos, Gibbs.

E. bifistulosum, Van Heurck.

Fl. Trop. Afr. viii. 239.

Mazoe, 4300 ft. April, Eyles.

E. lacteum, Rendle.

Fl. Trop. Afr. viii. 245 ; Cat. Afr. Pl. Welw. ii. 99.

Mashonaland, Bryce.

E. matopense, Rendle.

Journ. Linn. Soc. Bot. xxxvii. 475 .

Matopos, Gibbs.

E. submersum, Welw.

Fl. Trop. Afr. viii. 240 ; Cat. Afr. Pl. Welw. ii. 100.

Matopos, $4800 \mathrm{ft}$. April, Eyles, 65.

E. subulatum, N.E. Br.

Fl. Trop. Afr. viii. 255.

Victoria Falls, Kirk; May, Eyles, 125 ; Davy ; Sep. Gibbs, 175 ;

Engler ; July, Kolbe, 3141; Rogers, 5806.

830-Pæpalanthus Wahlbergii, Körn.

Fl. Trop. Afr. viii. 263 ; Fl. Cap. vii. 59 ; Cat. Afr. Pl. Welw. ii. 102.

Matopos, Sep. Gibbs, 86.

Family XXXIII.-COMMELINACEAE, Reichb.

896-Commelina africana, $L$.

Fl. Cap. vii. 9 ; Fl. Trop. Afr. viii. 45.

Salisbury, Rogers, 5805*.

C. Bainesii, C. B. Cl.

Fl. Trop. Afr. viii. 57 ; Cat. Afr. Pl. Welw. ii. 79.

Mangwe Riv. Baines.

C. benghalensis, $L$.

Fl. Trop. Afr. viii. 41; Fl. Cap. vii. 9; Cat. Afr. Pl. Welw. ii. 76.

Matabeleland, Elliott; Victoria Falls, Rogers, 7249*; Salisbury, Rogers, 5803*; Matopos, Rogers, 5636*. 
Genus No. C. Cecilae, C. B. $C l$.

Fl. Trop. Afr. viii. 51.

Gwelo, Cecil.

C. Forskalei, Vahl.

Fl. Trop. Afr. viii. 44 ; Cat. Afr. Pl. Welw. ii. 77.

Victoria Falls, Allen, 93 ; Matabeleland, Elliott.

C. krebsiana, Kunth.

Fl. Trop. Afr. viii. 47 ; Fl. Cap. vii. 10.

Matabeleland, Elliott.

C. Livingstoni, C. B. Cl.

Fl. Trop. Afr. viii. 59 ; Fl. Cap. vii. 11.

Matabeleland, Elliott.

C. nudiflora, $L$.

Fl. Trop. Afr. viii. 36 ; Fl. Cap. vii. 8 ; Cat. Afr. Pl. Welw. ii. 74.

Victoria Falls, Sep. Gibbs, 120.

C. spectabilis, C. B. Cl.

Fl. Trop. Afr. viii. 51; Cat. Afr. Pl. Welw. ii. 78.

Victoria Falls, Allen, 223.

C. zambesica, C. B. Cl.

Fl. Trop. Afr. viii. 43.

Victoria Falls, Rogers, 7249*, 7266*.

C. spp.

Victoria Falls, Allen, 282, cf. C. purpurea, C. B. Cl.

Victoria Falls, April, Flanagan, 3160.

Bulawayo, $4500 \mathrm{ft}$. Eyles, 100, cf. C. eckloniana, Kunth.

South Rhodesia, Rand, 251, 252, 253.

899--Aneilema æquinoctiale, Kunth. (A. adhaerens, Kunth.)

Fl. Trop. Afr. viii. 65; Fl. Cap. vii. 12 ; Nat. Pl. 284; Cat. Afr. Pl. Welw. ii. 79.

Bulawayo, $4600 \mathrm{ft}$. Dec. Eyles, 77 ; March, Monro; Salisbury to Bulawayo, Cecil, 81; Matabeleland, Elliott.

A. dregeana, Kunth.

Fl. Trop. Afr. viii. 70 ; Fl. Cap. vii. 13.

Gwelo, Jan. Gardner.

A. Johnstoni, K. Schum.

Fl. Trop. Afr. viii. 67.

Gwai Forest, Allen, 237 ; Nr. Luia Riv. Nicholson.

A. Nicholsoni, C. B. Cl.

Fl. Trop. Afr. viii. 70.

Nr. Luia Riv. Nicholson.

A. nyasense, $C . B . C l$.

Fl. Trop. Afr. viii. 66.

Odzani River Valley, Umtali, Teague, 124. 
A. sinicum, Lindl.

Fl. Trop. Afr. viii. 63 ; Fl. Cap. vii. 12 ; Nat. Pl. 294 ; Cat. Afr. Pl. Welw. ii. 79.

Malindi, Gwai Forest, 3600 ft. Allen, 249; Chirinda Forest, 3700-4000 ft. May, Swyn. 765 ; Odzani River Valley, Umtali, Teague, 120.

var. longifolia, C. B. Cl.

Salisbury, Cecil, 148.

\section{A. sp.}

South Rhodesia, Rand, 254.

904-Cyanotis fœcunda, Hassk.

Fl. Trop. Afr. viii. 80.

Matabeleland, Elliott.

C. nodiflora, Kunth.

Fl. Trop. Afr. viii. 82 ; Fl. Cap. vii. 14 ; Cat. Afr. Pl. Welw. ii. 80.

Inyanga, Cecil, 211 ; Bulawayo, March, Monro; Victoria Falls, Rogers, 5379.

908-Floscopa glomerata, Hassk.

Fl. Trop. Afr. viii. 86 ; Fl. Cap. vii. 15 ; Cat. Afr. Pl. Welw. ii. 80.

Victoria Falls, Sep. Gibbs, 297 ; Engler; Kirk; Rogers, 5080 ;

Chirinda, 3700 ft. fl. \& fr. June, Swyn. 764 ; Upper Buzi, 3000 ft. fl. \& fr. April, Swyn. 761; Odzani River Valley, Umtali, Teague, 209.

F. sp.

Victoria Falls, Rain Forest, Sep. Galpin, 7033, cf. F. glomerata, Hassk.

\section{Series LILIIFLORAE.}

FAMILy XXXVI.-JUNCACEAE, Vent.

936-Juncus Fontanesii, J. Gay.

Fl. Trop. Afr. viii. 94.

Matopos, fl. \& fr. Sep. Gibbs, 57.

FAMily XXXVIII.-LILIACEAE, Hall.

963-Gloriosa superba, $L$.

Fl. Trop. Afr. vii. 563 ; Fl. Cap. vi. 526 ; Cat. Afr. Pl. Welw. ii. 65. Bulawayo, $4500 \mathrm{ft}$. Jan. Eyles, 1054 ; Matopos, Marloth ; $4800 \mathrm{ft}$. Jan. Eyles, 32 ; Selukwe, Gardner, 37 ; Chipetzana Riv. 3000 ft. April, Swyn.; Chirinda, 3800 ft. Dec. Swyn. 6057, 6509 ; Victoria Falls, Rogers, 5622; South Rhodesia, Rand, 244 . 
Genus No.

G. virescens, Lindl. (G. simplex, L.)

Fl. Trop. Afr. vii. 563 ; Fl. Cap. vi. 526 ; Nat. Pl. 396 ; Cat. Afr. Pl. Welw. ii. 65.

Victoria Falls, Allen, 117; Odzani River Valley, Umtali, Teague, 2.

G. sp.

Mazoe, 4800 ft. Dec. Eyles, 493.

969-Androcymbium subulatum, Baker. (A. melanthioides, Willd. var.)

Fl. Trop. Afr. vii. 559; Fl. Cap. vi. 517.

Matabeleland, Oates; Umsweswe Riv. Baines.

973-0rnithoglossum glaucum, Salisb.

Fl. Trop. Afr. vii. 561 ; Fl. Cap. vi. 525.

Bulawayo, Eyles; Chubb, 389.

var. grandiflorum, Baker.

Fl. Trop. Afr. vii. 561 ; Fl. Cap. vi. 525.

Bulawayo, March, Monro.

989-Anthericum anceps, Baker.

Fl. Trop. Afr. vii. 482 ; Fl. Cap. vi. 382.

Matabeleland, Elliott.

A. elongatum, Willd. (A. filiforme, Thunb.)

Fl. Trop. Afr. vii. 491 ; Fl. Cap. vi. 389.

Bulawayo, 4500 ft. Nov. Eyles, 17 ; Chipetzana Riv. 3000 ft. April, Swyn. 773.

A. hispidum, $L$.

Fl. Cap. vi. 393.

Bulawayo, Rogers, 11323.

A. longistylum, Baker ex. descr.

Fl. Cap. vi. 381.

Bulawayo, Eyles, 572.

A. matabelense, Baker.

Fl. Trop. Afr. vii. 484.

Matengwe ? Riv. Holub.

A. Oatesii, Baker.

Fl. Trop. Afr. vii. 491 ; Journ. Bot. 1878, 324.

Matabeleland, Oates; Elliott.

A. recurvifolium, Baker.

Kew Bull. 1906, 28.

Salisbury, Cecil, 143.

A. rhodesianum, Rendle.

Journ. Linn. Soc. Bot. xl. 216.

Melsetter, 6000 ft. Oct. Swyn. 6056. 
Genus No.

A. spp.

Bulawayo, $4500 \mathrm{ft}$. Nov. Eyles, 9; Dec. Eyles, 1207.

Sebakwe, 4000 ft. Dec. Eyles, 102, cf. A. pubirachis, Baker.

Victoria, Monro, 903.

Victoria Falls, Kolbe, 4344.

990 -Chlorophytum andongense, Baker.

Fl. Trop. Afr. vii. 506 ; Cat. Afr. Pl. Welw. ii. 54.

Matopos, Rogers, 13801.

C. blepharophyllum, Schweinf.

Fl. Trop. Afr. vii. 501.

Chirinda, 3500 ft. Oct. Swyn. 329.

C. elatum, R. Br. var. Burchelli, Baker.

Fl. Cap. vi. 399.

Victoria Falls, Allen, 58.

C. gazense, Rendle.

Journ. Linn. Soc. Bot. xl. 216.

Chirinda Forest, 3700-4000 ft. fl. \& fr. April, May, Swyn. 318, 527.

C. polystachyum, Baker.

Fl. Trop. Afr. vii. 509.

Mazoe, $5200 \mathrm{ft.} \mathrm{Jan.} \mathrm{Eyles,} 521$.

C. sp.

Victoria, Monro, 629, cf. C. gallabatense, Schweinf.

1010-Schizobasis angolensis, Baker.

Fl. Trop. Afr. vii. 470; Cat. Afr. Pl. Welw. ii. 48.

Victoria, Monro, 442.

1011-Bowiea yolubilis, Harv.

Fl. Cap. vi. 367 ; Nat. Pl. 303 ; Harv. Gen. S.A. Pl. 401.

Mazoe, 4400 ft. fr. April, Eyles, 364 ; H. Jan. 556.

1012-Eriospermum Cecili, Baker.

Kew Bull. 1906, 28.

Inyanga, 6000-7000 ft. Cecil, 204.

E. spp.

Victoria, Monro, 784, cf. E. flexuosum, Welw.

Victoria, Monro, 433a, 439, cf. E. parvifolium, Jacq.

1024-Kniphofia rhodesiana, Renale.

Journ. Linn. Soc. Bot. xl. 214.

Nyahodi Riv. 5000 ft. April, Swyn. 723.

K. sp.

Salisbury, May, Flanagan, 3265, cf. K. Grantii, Baker.

1026-Aloe excelsa, Berger.

Matopos, Nov. Marloth, 3888; Gwanda, Noble, 69. 
Genus No.

A. rhodesiana, Rendle.

Journ. Linn. Soc. Bot. xl. 215.

Melsetter, 6000 ft. fl. \& fr. Oct. Swyn. 6047 ; Mt. Pene, 6000 ft. Swyn. 6048; Umvumvumvu Riv. Swyn.

A. Swynnertonii, Rendle.

Journ. Linn. Soc. Bot. xl. 215.

Chipetzana Riv. 3500 ft. April, Swyn.

\section{A. spp.}

Victoria, Monro, 339, cf. A. zebrina, Baker.

Victoria Falls, on island, Rogers, 5288.

Matopos, Rogers, 5361.

1047-Tulbaghia acutiloba, Harv.

Fl. Cap. vi. 404.

Victoria, Monro, 630.

T. alliacea, L. fil.

Fl. Trop. Afr. vii. 515 ; Fl. Cap. vi. 405.

Mazoe, 4300 ft. Dec. Eyles, 229.

T. campanulata, N.E. $B r$.

Mazoe, 4800 ft. Nov. Eyles, 194.

T. sp.

Salisbury, Rand, 1379.

1079-Albuca caudata, Jacq.

Fl. Trop. Afr. vii. 529 ; Fl. Cap. vi. 458.

Matopos, Oct. Gibbs, 287.

A. Melleri, Baker.

Fl. Trop. Afr. vii. 532.

Victoria Falls, Rogers, in Herb. Bolus, 13457.

A. tayloriana, Rendle.

Fl. Trop. Afr. vii. 530.

Victoria, Monro, 433b; Bulawayo, Chubb, 363*.

A. Wakefieldii, Baker.

Fl. Trop. Afr. vii. 531.

Bulawayo, March, Monro.

A. spp.

Bulawayo, March, Monro, cf. A. setosa, Jacq.

Victoria, Monro, 433c, 498, 584a.

Khami, Oct. Eyles, 1157.

Victoria Falls, Rogers, 5055, 5295.

1080-Urginea altissima, Baker.

Fl. Trop. Afr. vii. 538; Fl. Cap. vi. 470.

Bulawayo, Eyles; Victoria Falls, Allen, 52 ; Chirinda, 3800 ft. fl. \& fr. April, Swyn. 321a. 
Genus No.

\section{U. sanguinea, Schinz.}

Fl. Trop. Afr. vii. 538.

Victoria Falls, Sep. Galpin, 7051; fl. \& fr. Sep. Gibbs, 147 ;

Victoria, Monro, 469.

U. spp.

Bulawayo, March, Monro.

Victoria, Monro, 1026a, cf. U. comosa, Welw.

Victoria, Monro, 630, 839.

Victoria Falls, Rogers, 5294.

South Rhodesia, Rand, 250.

1084-Dipcadi anthericoides, Engl.

Matopos, $4700 \mathrm{ft}$. Jan. Eyles, 27.

D. hyacinthoides, Baker.

Fl. Cap. vi. 446.

Victoria, Monro, 440.

D. viride, Mcnch. (D. filamentosum, Medic.)

Fl. Trop. Afr. vii. 523 ; Fl. Cap. vi. 449 ; Nat. Pl. 239.

Bulawayo, March, Monro ; Chirinda, 3500-3800 ft. Oct. Swyn. 59.

D. spp.

Victoria, Monro, 625.

Victoria Falls, Rogers, 5398.

1086-Scilla ciliata, Baker.

Kew. Bull. 1906, 29.

Inyanga, Cecil, 165.

S. indica, Baker.

Fl. Trop. Afr. vii. 551.

Mt. Pene, 6500-7000 ft. Sep. Oct. Swyn. 767, 6055.

S. lanceæfolia, Baker.

Fl. Trop. Afr. vii. 557 ; Fl. Cap. vi. 487 ; Nat. Pl. 202 ; Cat. Afr. Pl. Welw. ii. 63.

Matopos, Oct. Gibbs, 191.

S. maesta, Baker.

Fl. Trop. Afr. vii. 552.

Mazoe, 4300-4800 ft. Nov. Eyles, 449.

S. rigidifolia, Kunth. (S. hispidula, Baker.)

Fl. Trop. Afr. vii. 549; Fl. Cap. vi. 481; Cat. Afr. Pl. Welw. ii. 61. Victoria, Monro, 464, 546; North Melsetter, 5000-6000 ft. Oct.

Swyn. 6053, 6226 ; Mt. Pene, 7000 ft. fl. \& fr. Oct. Swyn. 6054.

S. sp.

Victoria, Monro, 501, cf. S. tayloriana, Rendle.

1088-Eucomis undulata, Ait.

Fl. Cap. vi. 476.

Salisbury, Darling, in Herb. Bolus, 10792. 
Genus No.

E. zambesiaca, Baker.

Fl. Trop. Afr. vii. 528

Victoria, Monro, 858.

E. spp.

Mazoe, 4900 ft. Dec. Eyles, 495.

Salisbury, Mundy, ef. E. zambesiaca, Baker.

South Rhodesia, Rand, 247.

1090-Drimiopsis maculata, Lindl.

Fl. Cap. vi. 473; Nat. Pl. 304.

Bulawayo, March, Monro.

1109-Dracæna fragrans, Gawl.

Fl. Trop: Afr. vii. 440 ; Cat. Afr. Pl. Welw. ii. 47.

Chirinda Forest, 3700-4000 ft. Dec. Swyn. 724, 6520.

D. gazensis, Rendle.

Journ. Linn. Soc. Bot. xl. 214.

Chirinda, 3800 ft. Oct. Swyn.

D. spp.

Victoria, Monro, 347, 445.

1110-Sansevieria cylindrica, Bojer. (S. angolensis, Welw.)

Fl. Trop. Afr. vii. 335 ; Cat. Afr. Pl. Welw. ii. 25.

Victoria Falls, Galpin; Engler; Mangwe Riv. Baines.

S. senegalensis ?

South Rhodesia, Rand, 299.

S. spp.

Victoria Falls, Allen, 396, cf. S. sulcata, Bojer.

Victoria Falls, Rogers, 5059, 5287, ef. C. sulcata, Bojer.

1113-Asparagus æthiopicus, $L$.

Fl. Trop. Afr. vii. 434 ; Fl. Cap. vi. 271.

Chimanimani Mts. 7000 ft. Sep. Swyn. 1092.

A. africanus, Lam.

Fl. Trop. Afr. vii. 433 ; Fl. Cap. vi. 265; Cat. Afr. Pl. Welw.

ii. 42 .

Umtali, Engler.

A. angolensis, Baker.

Fl. Trop. Afr. vii. 432 ; Cat. Afr. Pl. Welw. ii. 42.

Chirinda, 3800 ft. May, Swyn. 520.

A. asiaticus, $L$.

Fl. Trop. Afr. vii. 432 ; Fl. Cap. vi. 265.

Chipetzana Riv. 3000 ft. Oct. Swyn. 239a; Upper Buzi, Swyn.;

Bulawayo, Eyles, 33.

A. falcatus, $L$.

Fl. Trop. Afr. vii. 435 ; Fl. Cap. vi. 271.

Chipete Forest, 3800 ft. April, Swyn. 91. 
A. laricinus, Burch.

Fl. Trop. Afr. vii. 433 ; Fl. Cap. vi. 267.

Matopos, by streams, Sep. Gibbs, 18 ; Gwelo, Jan. Gardner, 76.

A. medeoloides, Thunb.

Fl. Trop. Afr. vii. 435 ; Fl. Cap. vi. 272.

Chipete Forest, 3800 ft. fl. \& fr. April, May, Swyn. 89; Mt. Pene, 7000 ft. Swyn. 6058.

A. pilosus, Baker.

Fl. Trop. Afr. vii. 427.

Matabeleland, Oates.

A. plumosus, Baker.

Fl. Trop. Afr. vii. 430 ; Fl. Cap. vi. 260.

Umtali, Engler; Chirinda, 3700-4000 ft. Swyn. 240.

A. racemosus, Willd.

Fl. Trop. Afr. vii. 434 ; Fl. Cap. vi. 269 ; Cat. Afr. Pl. Welw. ii. 43.

Victoria Falls, Allen, 6; Gwai, Engler; South Rhodesia, Rand, 242.

A. stipulaceus, Lam.

Fl. Cap. vi. 264.

Bulawayo, Rogers, 4486.

A. virgatus, Baker.

Fl. Trop. Afr. vii. 426 ; Fl. Cap. vi. 259.

Chipete, 3800 ft. Oct. Swyn. 239.

\section{A. spp.}

Bulawayo, Jan. Gardner, 74, cf. A. bechuanicus, Baker.

Bulawayo, Rand, 545.

Gwelo, Jan. Gardner, 75, ef. A. bechuanicus, Baker.

Victoria, Monro, 333, 1000, cf. A. stipulaceus, Lam.

South Rhodesia, Rand, 404, 405.

1147-Behnia reticulata, Didr.

Fl. Cap. vi. 274.

Chimanimani Mts. 7000 ft. Sep. Swyn. 1098; Mt. Pene, 7000 ft. Swyn. 6057 ; Chirinda Forest, 4000 ft. fl. \& fr. Oct. Swyn. 90 ;

Chipete Forest, fl. \& fr. Oct. Swyn.

1149-Hymenocallis senegambica, Kunth. \& Bouché.

Fl. Trop. Afr. vii. 408.

Victoria Falls, Rogers, 7258*.

1151-Smilax kraussiana, Meisn.

Fl. Trop. Afr. vii. 424 ; Fl. Cap. vi. 256 ; Nat. Pl. 339 ; Cat. Afr. Pl. Welw. ii. 41.

Victoria Falls, Palm Kloof, Engler; Chirinda, 3500-5900 ft. fl. \& fr. Nov. Dec. Swyn. 212; Chimanimani Mts. 7000 ft. Swyn. 1099 ; Melsetter Dist. common, Swyn. 


\section{FAMILy XL.-AMARYLLIDACEAE, Lindl.}

Genus No.

1167-Hæmanthus Cecilae, Baker.

Kew Bull. 1906, 28.

Between Salisbury and Bulawayo, Cecil, 76.

H. multiflorus, Martyn.

Fl. Trop. Afr. vii. 388 ; Cat. Afr. Pl. Welw. ii. 34.

Matabeleland, Oates; Victoria Falls, Allen, 44; Bulawayo, March, Monro; Chirinda, 3800 ft. Oct. Swyn. 359.

H. zambesiacus, Baker.

Fl. Trop. Afr. vii. 387.

Salisbury, Darling, in Herb. Bolus, 11197.

1168-Buphane disticha, Herb. (B. toxicaria, Herb.)

Fl. Trop. Afr. vii. 392 ; Fl. Cap. vi. 242 ; Nat. Pl. 595 ; Cat. Afr.

Pl. Welw. ii. 35.

Matopos, Oct. Gibbs, 208; Salisbury, Mundy, 934.

1177-Brunsvigia, sp.

Mazoe, 4800 ft. Sep. Eyles, 551, cf. B. Kirkii, Baker.

South Rhodesia, Rand, 224, 654.

1189-Crinum Harmsii, Baker.

Victoria Falls, Allen, 43.

C. Kirkii, Baker.

Fl. Trop. Afr. vii. 402.

Chirinda, $3800 \mathrm{ft}$. Swyn.

C. zeylanicum, $L$.

Fl. Trop. Afr, vii. 401.

Victoria Falls, Allen, 136.

C. spp.

Sebakwe, 4000 ft. Dec. Eyles, 170.

South Rhodesia, Rand, 228.

1190-Ammocharis falcata, Herb.

Fl. Cap. vi. 204.

Bulawayo, Chubb, 7.

1191-Cyrtanthus angustifolius, Ait.

Fl. Cap. vi. 223 ; Nat. Pl. 5.

Mazoe, $4400 \mathrm{ft}$. Sep. Eyles, 416.

C. rhodesianus, Rendle.

Journ. Linn. Soc. Bot. xl. 211.

Chimanimani Mts. 7000 ft. Sep. Oct. Swyn. 769.

1202-Pancratium trianthum, Herb. (P. Chapmanni, Harv.)

Fl. Trop. Afr. vii. 407 ; Harv. Gen. S.A. Pl. 384.

Chirinda, 3800 ft. Swyn. 322 ; Victoria Falls, Rogers, 7470*, $7544 *, 8671 *$. 
Genus No.

P. sp.

Bulawayo, 4500 ft. Nov. Eyles, 1248, cf. P. trianthum, Herb.

1208-Hippeastrum equestre, Herb.

Victoria Falls, Allen.

1230-Hypoxis angustifolia, Lam.

Fl. Trop. Afr. vii. 378 ; Fl. Cap. vi. 180.

Victoria, Monro, 866.

H. rigidula, Baker.

Fl. Cap. vi. 186 ; Nat. Pl. 552.

Bulawayo, 4500 ft. Jan. Eyles, 59 ; Mazoe, 4300-4800 ft. Nov. Eyles, 455.

var. pilosissima, Baker.

Fl. Cap. vi. 186.

Bulawayo, Jan. Eyles.

H. Rooperii, Moore.

Fl. Cap. vi. 188.

Salisbury, Darling.

H. villosa, $L$. fil.

Fl. Trop. Afr. vii. 379 ; Fl. Cap. vi. 184.

Matabeleland, Oates; Matopos, Oct. Gibbs, 192 ; Victoria, Monro, 654 ; North Melsetter, 5000-6000 ft. Oct. Swyn. 6215; Mt. Pene, 6000-7000 ft. Sep. Oct. Swyn. 770, 6082, 6204; Chirinda, 3800 ft. May, Oct. Swyn. 332 ; Marendellas? Rogers, 4043.

\section{H. spp.}

South Rhodesia, Rand, 225, 226, 227, 237.

1231-Walleria nutans, Kirk.

Fl. Trop. Afr. vii. 568 ; Fl. Cap. vi. 528.

Bulawayo, $4500 \mathrm{ft}$. Dec. Eyles, 39.

\section{W. sp.}

South Rhodesia, Rand, 246.

\section{Family XLI.-VELLOZIACEAE, Drude.}

1246-Vellozia equisetoides, Baker. (Barbacenia equisetoides, Engl.)

Fl. Trop. Afr. vii. 411 ; Fl. Cap. vi. 245.

Semokwe ? Riv. Baines; Matabeleland, Scott-Elliott ; Victoria Falls, Allen, 41; Matopos, Engler ; 5000 ft. Jan. Eyles, 25 ; Mazoe, 5000 ft. Oct. Eyles, 439 ; 4700 ft. Jan. Eyles, 516 ?; South Rhodesia, Rand, 270 ; Odzani River Valley, Umtali ; Teague, 236. 
Genus No. $\mathbf{Y}$. humilis, Baker.

Fl. Trop. Afr. vii. 409 ; Fl. Cap. vi. 246.

Fig-tree, Dec. Eyles, 157 ; Matabeleland, Elliott.

\section{Y. retinervis, Baker.}

Fl. Cap. vi. 244.

Matopos, Nov. Marloth, 3415.

\section{Y. spp.}

Victoria Falls, Galpin, 7058.

Mazoe, 4300-5000 ft. Oct. Eyles, 440, cf. V. cquatorialis, Rendle.

Mazoe, 5300 ft. Jan. Eyles, 517, cf. V. acuminata, Baker.

Goromanzi, Mundy, cf. V.retinervis, Baker.

Victoria, Monro, 800, cf. V. trichophylloides, Hemsl.

Victoria, Monro, 801.

Salisbury, Mundy.

Harvest Valley, Rogers.

\section{FAMILY XLII.-TACCACEAE, Lindl.}

1248-Tacca pinnatifida, Forst.

Fl. Trop. Afr. vii. 413 ; Cat. Afr. Pl. Welw. ii. 36.

Victoria Falls, on island, Allen, 251; Salisbury and Umtani, Rogers, 5767\%.

\section{FAnily XLIII.-DIOSCOREACEAE, Lindl.}

1252-Dioscorea beccariana, Mart.

Fl. Trop. Afr. vii. 420.

Gwai Forest, 3800 ft. Allen, 232 ; Victoria Falls, Rogers, 5381 ;

Chirinda, 3800 ft. Dec. Swyn. 1091a, 6630; Bulawayo, Chubb, 18.

D. Buchanani, Benth.

Fl. Trop. Afr. vii. 415.

Chirinda, $3800 \mathrm{ft}$. July, Swyn. 1091.

P. dumetorum, Pax. (D. quartiniana, A. Rich.)

Fl. Trop. Afr. vii. 419; Cat. Afr. Pl. Welw. ii. 40.

Bulawayo, 4500 ft. Dec. Eyles, 1141 ; Chirinda, $3700 \mathrm{ft}$. young fr. March, Swyn. 215.

D. sativa, $L$.

Fl. Trop. Afr. vii. 415 ; Cat. Afr. Pl. Welw. ii. 38.

Chirinda Forest, 3800 ft. (wild), May, Swyn. 531.

D. schimperiana, Hochst.

Fl. Trop. Afr. vii. 419 (var.).

Chirinda, 3700-4000 ft. fl. \& fr. Feb. Swyn. 214 ; Chipete, Swyn. 
Genus No.

var. vestita, $\operatorname{Pax}$.

Fl. Trop. Afr. vii. 419.

Mazoe, 4300 ft. Jan. Eyles, 503.

D. sylvatica, Eckl. (Testudinaria sylvatica, Benth.)

Fl. Cap. vi. 253.

Mazoe, 4300 ft. Dec. Eyles, 212 ; Bulawayo, Chubb, 9.

D. spp.

Victoria Falls, Rogers, $5372^{\text {I }}$, cf. D. rubiginosa, Benth.

South Rhodesia, Rand, 432.

Family XLIV.-IRIDACEAE, Lindl.

1265-Moræa edulis, Ker.

Fl. Cap. vi. 20.

Victoria, Monro, 677.

M. iridioides, $L$.

Fl. Trop. Afr. vii. 342 ; Nat. Pl. 299.

Chirinda Forest, 3700-4000 ft. fl. \& fr. Oct. Dec. Swyn. 399, 6508; Mt. Pene, 7000 ft. Oct. Swyn. 6067; Chipete Forest, Swyn.

\section{M. spp.}

Bulawayo, Jan. Gardner, 2; Victoria, Monro, 601 ; Matopos, 5000 ft. Nov. Eyles, 1144 ; Salisbury, Mundy ; South Rhodesia, Rand, 236.

1272-Ferraria Randii, Rendle. (Morca Randii, Rendle.)

Journ. Bot. 1898, 144.

South Rhodesia, Rand, 233 ; Bulawayo, 4500 ft. Jan. Eyles, 158.

1284-Bobartia sp.

Victoria, Monro, 755.

1295-Aristea compressa, Buching.

Fl. Cap. vi. 50.

North Melsetter, 6000 ft. fl. \& fr. Oct. Swyn. 6060.

A. zombensis, Baker.

Fl. Trop. Afr. vii. 346.

Chipete Forest, 3700-4000 ft. fr. Oct. Swyn. 399a; Chipete Forest, 3800 ft. fr. April, Swyn. 399.

1301-Hesperantha matopensis, Gibbs.

Journ. Linn. Soc. Bot. xxxvii. 471.

Matopos, Sep. Gibbs, 44.

1303-Dierama pendula, Baker.

Fl. Trop. Afr. vii. 349.

Melsetter, 6000 ft. Oct. Swyn. 6064, 6065.

I Rogers has this plant named D. rubiginosa, Benth. 
Genus No.

1306-Tritonia aurea, Pappe. (Crocosmia aurea, Planch.)

Fl. Trop. Afr. vii. 355 ; Fl. Cap. vi. 129 ; Nat. Pl. 519.

Chirinda, 3600 ft. March, Swyn. 323.

1310-Babiana Bainesii, Baker.

Fl. Cap. vi. 107.

Matopos, 5000 ft. April, Eyles, 38; March, Flanagan, 2949;

Salisbury, May, Flanagan, 3249.

1311-Gladiolous atropurpureus, Baker.

Fl. Trop. Afr. vii. 364.

Victoria Falls, Allen, 198.

G. brevicaulis, Baker.

Fl. Trop. Afr. vii. 366; Cat. Afr. Pl. Welw. ii. 28.

Victoria, Monro, 647.

G. brevifolius, Jacq.

Fl. Cap. vi. 143.

Matabeleland, Oates.

G. dracocephalus, Hook $f$.

Fl. Cap. vi. 157.

Matopos. March, Flanagan, 2966.

G. gazensis, Rendle.

Journ. Linn. Soc. Bot. xl. 210.

Melsetter, 6000 ft. Swyn.; Chirinda, 3700-4000 ft. Swyn.; Mt.

Pene, 6500-7000 ft. Swyn. ; in fl. Sep. Swyn. 779.

G. Melleri, Baker.

Fl. Trop. Afr. vii. 362.

Matopos, Oct., Gibbs, 202 ; Mazoe, 4400 ft. Sep. Eyles, 417 ;

Matabelelanḋ, Oates; Salisbury, Rogers, 4057; Victoria,

Monro, 485.

G. Oatesii, Rolfe.

Fl. Trop. Afr. vii. 373.

Matabeleland, Oates; Victoria Falls, Allen, 193 ; Bulawayo, Jan.

Gardner, 78.

G. permeabilis, Delar.

Fl. Cap. vi. 162.

Mazoe, 4300 ft. Dec. Eyles, 488 ; Bulawayo, Chubb, 11.

G. primulinus, Baker.

Victoria Falls, Allen, 18; Sep. Gibbs, 323 ; Rogers, 5397;

Monro.

G. quartinianus, A. Rich.

Fl. Trop. Afr. vii. 371 ; Cat. Afr. Pl. Welw. ii. 29.

Bulawayo, March, Monro. 
Genus No.

\section{G. spp.}

Salisbury, May, Flanagan, 3252 ; Rand, 541.

Victoria, Monro, 967, ef. G. psittacinus, Hook.

Victoria, Monro, 1091.

Bulawayo, 4500 ft. Nov. Eyles, 1224.

South Rhodesia, Rand, 230, 234, 235, 656.

\section{2-Antholyza sp.}

Victoria, Monro, 301.

1314-Lapeyrousia caudata, Schinz.

Fl. Trop. Afr. vii. 352.

South Rhodesia, Rand, 229.

L. cruenta, $B k r$.

Fl. Cap. vi. 96 ; Fl. Trop. Afr. vii. 354.

Bulawayo, Rogers, 13586*.

L. cyanescens, Baker.

Fl. Trop. Afr. vii. 354 ; Cat. Afr. Pl. Welw. ii. 28. South Rhodesia, Rand, 232.

L. grandiflora, Baker.

Fl. Trop. Afr. vii. 355 ; Fl. Cap. vi. 96 ; Nat. Pl. 526.

Bulawayo, Dec. Eyles; Mazoe, 4300-5300 ft. Jan. Eyles, 1134.

L. odoratissima, Baker.

Fl. Trop. Afr. vii. 354 ; Cat. Afr. Pl. Welw. ii. 28.

Victoria Falls, Rogers, 5395.

L. porphyrosiphon, Baker.

Fl. Trop. Afr. vii. 353.

Victoria Falls, Allen, 146 ; Rogers, 5415.

L. rhodesiana, N. E. Br.

Kew Bull. 1906, 169.

Between Salisbury and Umtali, Cecil, 154.

L. Sandersoni, Baker.

Fl. Trop. Afr. vii. 352 ; Fl. Cap. vi. 95.

Bembesi, Jan. Gardner, 7 ; Victoria Falls, Rogers, 5536 ; Victoria, Monro, 678, 674 ; Bulawayo, Rogers, common ; Matabeleland, Scott-Elliott.

L. Welwitschii, Baker.

Fl. Trop. Afr. vii. 352 ; Cat. Afr. Pl. Welw. ii. 27.

Matopos, 5000 ft. Feb. Eyles, 1173.

L. spp.

Bulawayo, 4500 ft. Jan. Eyles, 75 ; Nov. 91.

South Rhodesia, Rand, 231. 


\section{SERIES SCITAMINEAE.}

Family XLV.-MUSACEAE, J. St. Hil.

Genus No.

1318-Musa Ensete, Gmel.

Fl. Trop. Afr. vii. 329.

Marandellas, Engler.

Family XLVI.-ZINGIBERACEAE, L. C. Rich.

1329-Kæmpferia Carsoni, Baker.

Fl. Trop. Afr. vii. 296.

Victoria Falls, Allen, 104.

K. Kirkii, K. Schum. var. elatior, Stapf.

Fl. Trop. Afr. vii. 294 (type).

Salisbury, H. J. Elwes, F.R.S., see Kew Bull. 1908, 196.

K. sp.

Victoria Falls, Rogers, 5390.

1344-Amomum sp.

Salisbury, Darling, in Herb. Bolus, 10793.

FAMILy XLVII.-CANNACEAE, Link.

1363-Canna indica, $L$., subsp. C. orientalis, Rosc.

Fl. Trop. Afr. vii. 328 ; Cat. Afr. Pl. Welw. ii. 24.

Victoria, Monro, 736, 1044 ; Chirinda, 3800 ft. March, Swyn.

398 ; Matabeleland, Rand.

\section{SERIES MICROSPERMAE.}

Family XLIX.-BURMANNIACAE, Blume.

1382-Burmannia bicolor, Mart. var. africana, Ridl.

Fl. Trop. Afr. vii. 11 ; Cat. Afr. Pl. Welw. ii. 2.

Matopos, 5000 ft. Eyles, 52.

Family L.-ORCHIDACEAE, Lindl.

1408-Holothrix grandiflora, Reichb. $f$.

Fl. Cap. V. iii. 110.

Umtali, Engler. 
Genus No

H. Randii, Rendle.

Journ. Bot. 1899, 208.

Salisbury, Sep. Rand, 596.

1422-Habenaria antennifera (Rolfe), Torre \& Harms. (Bcnatea antenniferc, Rolfe.)

Fl. Cap. V. iii. 142.

Bulawayo, March, Monro.

H. malacophylla, Reichb. $f$.

Fl. Trop. Afr. vii. 230 ; Fl. Cap. V. iii. 126.

Victoria Falls, 3000 ft. Rain Forest, May, Eyles, 92 ; Allen, 4 ; Flanagan, 3299 ; Rogers, 5129.

H. pedicellaris, Reichb. $f$.

Fl. Trop. Afr. vii. 244.

Upper Buzi, 3000 ft. April, Swyn. 744 ; South Rhodesia, Rand, 269.

H. Rehmanni, Bolus.

Fl. Cap. V. iii. 129.

Victoria, Monro, 904, 890, 865, 868.

H. spp.

Victoria Falls, Allen, 139, cf. H. subarmata, Reichb. f.

Mazoe, 4400 ft. Dec. Eyles, 499, cf. H. Galpinii, Bolus.

Matopos, 5000 ft. March, Eyles, 1037, 1064.

1428--Brachycorythis acutiloba, Rendle.

Journ. Linn. Soc. Bot. xl. 208.

Chirinda, 3800 ft. Dec. Swyn. 6632.

B. hispidula?

South Rhodesia, Rand, 266.

1430-Satyrium Buchananii, Schlechter.

Fl. Trop. Afr. vii. 572.

Lusitu Riv. 5000 ft. April, Swyn. 754 ; South Rhodesia, Rand, 267.

S. macrophyllum, Lindl.

Fl. Cap. V. iii. 166 ; Orchids of S.A. i. 74.

Mazoe, 4300 ft. April, Eyles, 305; March, Flanagan, 3027 ;

Matopos, $5000 \mathrm{ft}$. March, Eyles, 1036.

S. speciosum, Rolfe.

Fl. Trop. Afr. vii. $270 \& 574$.

Chirinda, 3800 ft. Dec. Swyn. 331 ; Upper Buzi, 3000 ft. April, Swyn. 745.

S. trinerve, Lindl. (S. occultum, Rolfe.)

Fl. Trop. Afr. vii. 273 ; Cat. Afr. Pl. Welw. ii. 16 ; Orchids of S.A. ii. 61.

Chirinda, 3800 ft. Jan. Swyn. 750. 
Genus No.

1431-Schizochilus Cecili, Rolfe.

Kew Bull. 1906, 168.

Inyanga, Cecil, 202.

1434 -Disa equestris, Reichb. $f$.

Fl. Trop. Afr. vii. 284 ; Cat. Afr. Pl. Welw. ii. 18.

Rhodesia, Lord Rothschild, see Kew Bull. 1908, 86; South Rhodesia, Rand, 268.

D. hamatopetala, Rendle.

Fl. Trop. Afr. vii. 286.

Mt. Pene, 6500-7000 ft. fl. \& fr. Sep. Swyn. 753.

1565-Polystachya Hislopii, Rolfe.

Kew Bull. 1914, 375.

Rusapi, A. Hislop.

1568-Ansellia africana, Lindl.

Fl. Trop. Afr. vii. 101 ; Cat. Afr. Pl. Welw. ii. 7 ; Orchids of S.A. ii. 29.

Matopos, Sep. Oct. Gibbs, 270, epiphytic ; Engler.

A. humilis, Bull.

Fl. Trop. Afr. vii. 102.

Victoria Falls, Allen, 45.

A. spp.

Gatooma, Rogers, 5521, ef. A. confusa, N. E. Br.

Victoria Falls, Rogers, 7235, cf. A. confusa, N. E. Br.

1631-Calanthe natalensis, Reichb. $f$.

Victoria Falls, Engler; Rogers, 5697.

C. sanderiana?

Victoria Falls, Allen, 140.

C. sp.

Salisbury, March, Flanagan, 3016, in marshy places.

1647-Lissochilus arenarius, Lindl. (L. dilectus, Reichb. f. Eulophia aienaria, Bol.)

Fl. Trop. Afr. vii. 82 ; Fl. Cap. v. iii. 61 ; Cat. Afr. Pl. Welw. ii. 5 .

Matabeleland, Oates; Victoria Falls, Allen, 88; Chirinda, 3500 ft. Nov. Swyn. 751; South Rhodesia, Rand, 262 ; Odzani River Valley, Umtali, Teague.

L. Buchanani, Reichb.f. (Eulophia Buchananii, Bolus.)

Fl. Cap. V. iii. 60; Orchids of S.A. ii. 23.

Matopos, Rogers, 5656 ; Victoria, Jan. Miss Krige, in Herb.

Bolus, 10715; Victoria Falls, Rogers, 13070*.

L. Eylesii, Rendle.

Journ. Bot. 1905, 53.

Matopos, 5000 ft. Feb. Eyles, 150. 
L. livingstonianus, Reichb. $f$.

Fl. Trop. Afr. vii. 81.

Mazoe, 4300-4800 ft. Nov. Eyles, 445; Victoria, Monro, 785.

L. microceras, Reichb. $f$.

Fl. Trop. Afr. vii. 74.

Umtali, Engler.

L. milanjianus, Rendle.

Fl. Trop. Afr. vii. 98.

Umtali, Engler.

L. Oatesii, Rolfe.

Fl. Trop. Afr. vii. 89.

Matabeleland, Oates.

L. papilinaceus, Rendle.

Fl. Trop. Afr. vii. 91.

Chirinda, 3700 ft. March, Swyn. 227.

L. Wakefieldii, Reichb. f. \& S. Moore.

Fl. Trop. Afr. vii. 95 ; Fl. Cap. V. iii. 60.

Victoria Falls, Allen, 70.

1648-Eulophia clitellifer, Bolus. (Lissochilus clitellifer, Reichb. f.)

Fl. Cap. V. iii. 55.

Mazoe, 4300-4800 ft. Sep. Eyles, 418; Umtali, Engler ; Victoria, Monro, 461, 438.

E. dregeana, Lindl.

Fl. Cap. V. iii. 36 ; Orchids of S.A. ii. 9.

Mazoe, 4800-5100 ft. Dec. Eyles, 497.

E. Krebsii, Bolus. (Lissochilus Krebsii, Reichb. f.)

Fl. Trop. Afr. vii. 91 ; Fl. Cap. V. iii. 58.

Matopos, Oct. Gibbs, 254 ; Mazoe, 4500 ft. Dec. Eyles, 480 ;

Salisbury, Rand, 1377 ; South Rhodesia, Rand, 263; Odzani

River Valley, Umtali, Teague.

E. milanjiana, Rendle.

Fl. Trop. Afr. vii. 63.

Chirinda, 3800 ft. Oct. Swyn. 328.

E. saccatus, Rendle. (Lissochilus saccatus, Rendle.)

Fl. Trop. Afr. vii. 98.

South Rhodesia, Rand, 647.

E. speciosa, Bolus. (Lissochilus speciosus, R. Br.)

Fl. Cap. V. iii. 59 ; Orchids of S.A.

Mazoe, 4800 ft. Nov. Eyles, 463.

E. Swynnertonii, Rendle.

Journ. Linn. Soc. Bot. xl. 207.

Mt. Pene, 6500-7000 ft. Sep. Oct. Swyn. 752, 6050. 
Genus No.

\section{E. undulata?}

Bulawayo, March, Monro, n. sp.? ined.

E. Welwitschii, Rolfe.

Fl. Trop. Afr. vii. 61; Cat. Afr. Pl. Welw. ii. 4.

South Rhodesia, Rand, 261.

E. spp.

Gwelo, Jan. Gardner, 27.

Salisbury, Rand, 542, 543.

Mazoe, 4300-4800 ft. Dec. Eyles, 474, 491.

Matopos, $5000 \mathrm{ft}$. Nov. Eyles, 1226, 1227.

Victoria, Monro, 476, 899.

Victoria, Monro, 661, cf. E. Shupangae, Kränzl.

South Rhodesia, Rand, 264, 265.

1651-Pteroglossaspis sp.

Mazoe, 4300 ft. Jan. Eyles, 501.

1822_Saccolabium sp?.

Mazoe, 4700 ft. Dec. Eyles, 476.

1828-Angrecum leonis ?.

Mazoe, $4700 \mathrm{ft}$. Nov. Eyles, 465.

A. rhodesianum, Rendle.

Journ. Linn. Soc. Bot. xl. 208.

Melsetter, 6000 ft. fr. Sep. Swyn. 755, epiphytic on Brachystegia

Randii.

1837-Mystacidium sp.

Mazoe, 4700-5000 ft. Nov. Eyles, 466.

Class DICOTYLEDONEAE.

Sub-Class ARCHICHLAMYDEAE.

Series PIPERALES.

FAMily LIII.-PIPERACEAE, L. C. Rich.

1862-Piper capense, $L$.

Fl. Trop. Afr. VI. i. 146.

Chirinda Forest, 3700-4000 ft. June, Swyn. 66; Mt. Pene,

$6500 \mathrm{ft}$. Swyn. 1097.

1866-Peperomia brachytrichoides, Engl.

Victoria Falls, in Palm Kloof, Engler.

P. mascharena, C. DC.

Chipete Forest, 3800 ft. May, Swyn. 426 ; Chirinda Forest, Swyn. 
Genus No.

P. reflexa, A. Dietr.

Fl. Trop. Afr. VI. i. 155.

Chipete Forest, 3800 ft. May, Swyn. 426a ; Chirinda Forest, Swyn.

\section{P. spp.}

Victoria Falls, in Palm Kloof, April, Flanagan, 3298.

Melsetter, Johnson, 181, cf. P. arabica, C. DC.

\section{Series SALICALES.}

FAmily LVI.-SALICACEAE, Lindl.

1873-Salix capensis, Thunb.

For. Fl. Cape, 328.

Matopos, Sep. Gibbs, 21; March, Flanagan, 2953 ; Mazoe, 4300 ft. April, Eyles, 316 ; Govt. Herb. 924 ; Odzani River Valley, Umtali, Teague, 125.

S. ramiflora, $R$. v. Seem.

Victoria Falls, Engler.

S. Safsaf, Forsk.

Victoria Falls, Oct. Davy.

S. spp.

South Rhodesia, Rand, 219, 403.

Series MYRICALES.

FAMILy LVII.-MYRICACEAE, Lindl.

1874-Myrica æthiopica, $L$.

For. Fl. Port. E. Afr. 107; For. Fl. Cape, 330.

Victoria Falls, on island, Engler; Rogers, 7454; Odzani River Valley, Umtali, Teague, 109.

M. pilulifera, Rendle.

Mt. Pene, 6500 ft. fl. July, fr. Sep. Swyn. 530, 1735.

var. puberula Rendle.

Journ. Bot. 1903, 86.

Melsetter, 6000 ft. fr. Sep. Oct. Swyn. 610, 610a; Nyahodi Riv. 5500 ft. Swyn. 623 ; Mt. Pene, 7000 ft. Swyn. 6084.

\section{SERIES URTICALES.}

FAmily LXIII.-ULMACEAE, Mirb.

1898-Celtis dioica, S. Moore.

Journ. Linn. Soc. xl. 204.

Chirinda Forest, 3700-4000 ft. Oct. Swyn. 108, fr. May. 
C. rhamnifolia, Presl. (C. kraussiana, Bernh.)

Nat. Pl. 28 ; For. Fl. Cape, 306.

Mazoe, 4600 ft. Aug. Eyles, 399.

C. spp.

Gwanda, Noble, 62.

Lomagundi, Govt. Herb. 993.

1902_Trema guineensis, Priemer.

Chirinda, 3800 ft. April, Swyn. 129.

\section{FAmily LXIV.-MORACEAE, Lindl.}

1960-Bosquiea Phoberos, Baill.

Chipete Forest, 3800 ft. Swyn. ; Chirinda Forest, 3500 ft. Swyn. 1961-Ficus caffra, Miq.

Victoria Falls, Rogers, 5978, 7464, 7455.

F. capensis, Thunb.

For. Fl. Port. E. Afr. 101; For. Fl. Cape, 307 ; Cat. Afr. Pl. Welw. i. 1016.

Victoria Falls, Engler; Rogers, 5984 ; Chirinda Forest, 37004000 ft. early fr. June, Swyn. 434; Melsetter, Swyn.

F. capreæfolia, Del.

Sabi Riv. 1000 ft. fr. Dec. Swyn. 1101.

F. exasperata, Vahl.

Cat. Afr. Pl. Welw. i. 1010.

Chirinda Forest, 3700-4000 ft. fl. \& fr. Aug. Oct. Swyn. 601.

F. lutea, Vahl.

Matopos, fr. Oct. Gibbs, 285; Rogers, 5411; Victoria Falls, Engler; Umtali, Engler.

F. maschonae, Warb.

Umtali, Engler.

F. matabelae, Warb.

Matopos, Engler; Salisbury, Engler.

F. natalensis, Hochst.

For. Fl. Port. E. Afr. 98 ; For. Fl. Cape, 307.

Matopos, Nov. Marloth, 3404.

F. Rehmannii, Warb.

Matopos, Engler.

F. rhodesiaca, Warb.

Salisbury, Engler.

F. salicifolia, Vahl. var. australis, Warb.

Matopos, Engler.

F. Sonderi, Miq.

Matopos, fr. Oct. Gibbs, 26 ; March, Flanagan, 2977 ; Rogers, 5251 ; Bulawayo, Rogers, 6203. 
Genus No.

var. villosa, Warb.

South Rhodesia, Marloth.

F. subcalcarata, Warb. \& Schweinf. South Melsetter, Swyn.

F. Victoriae, Warb.

Victoria Falls, Engler.

\section{F. spp.}

Victoria Falls, April, Flanagan, 3303, cf. F. Victoriae, Warb.

Victoria Falls, Allen, 183 ; Rogers, 5125.

Salisbury, March, Flanagan, 3017, cf. F. lutea, Vahl.

Salisbury, Rand, 537, 538.

Victoria, Monro, 361, cf. F. trachyphylla, Fenzl.

Victoria, Monro, 390.

Matopos, Rogers, 5198, cf. F. caffra, Miq.

Matopos, Rogers, 5354, 5357 ; Govt. Herb. 899.

Lomagundi, Govt. Herb. 975.

South Rhodesia, Rand, 398, 399.

1966-Myrianthus arboreus, Beauv.

Cat. Afr. Pl. Welw. i. 995.

Chirinda Forest, 3700-4000 ft. Sep. Swyn. 111, 111a; Mt. Pene, 6500-7000 ft. Dec. Swyn. 1052.

\section{FAMILY LXV-URTICACEAE, Endl.}

1978-Urera obovata, Benth.

Cat. Afr. Pl. Welw: i. 986.

Chirinda Forest, 3700-4000 ft. Jan. Swyn. 1518.

1982-Fleurya capensis, Wedd.

Nat. Pl. 577.

Chirinda Forest, 3700-4000 ft. fl. April, fr. May, Swyn. 338, 793. 1992-Pouzolzia hypoleuca, Wedd.

Victoria, Monro, 645, 892 ; Bulawayo, Chubb, 11a; Matopos, Rogers, 5362.

\section{SERIES PROTEALES.}

Family LXVI.-PROTEACEAE, J. St. Hil.

2034-Faurea racemosa, Farmar.

Fl. Trop. Afr. VI. i. 208; Kew Bull. 1908, 58.

Chimanimani Mts. 7000 ft. Sep. Swyn. 639; Mt. Pene, 7000 ft. Oct. Swyn. 6096. 
Genus No.

F. saligna, Harv.

Fl. Trop. Afr. VI. i. 209 ; Fl. Cap. V. i. 640 ; Cat. Afr. Pl. Welw. i. 921 ; For. Fl. Cape, 297.

Fig-tree, 4500 ft. Dec. Eyles, 156; Matopos, Marloth; Hutchins; Oct. Davy; Oct. Gibbs, 313 ; Engler ; Rusapi, Engler; Victoria, Monro, 782; 617; Kocwe Hills, Govt. Herb. 953; Chirinda, 3500 ft. Sep. Swyn. 42 ; Chimanimani Mts. $7000 \mathrm{ft}$. Nov. Swyn. 1796 ; Odzani River Valley, Umtali, Teague, 139.

E. speciosa, Welw.

Fl. Trop. Afr. VI. i. 211; Fl. Cap. V. i. 642 ; Cat. Afr. Pl. Welw. i. 922.

Mazoe, 4800 ft. Sep. Eyles, 415 ; Salisbury, March, Flanagan, 3005 ; Melsetter, 6000 ft. Sep. Oct. Swyn. 625, 626 ; between Melsetter and Umvumvumvu Riv. Swyn.; Odzani River Valley, Umtali, Teague, 138.

var. lanuginosa, Hiern.

Cat. Afr. Pl. Welw. i. 922.

Salisbury, Engler.

F. usambarensis, Engl.

Fl. Trop. Afr. VI. i. 210.

Umtali, Engler.

2035-Protea abyssinica, Willd.

Fl. Trop. Afr. VI. i. 199 ; Fl. Cap. V. i. 581.

Matopos, Galpin, 7090 ; fl. \& fr. Oct. Gibbs, 59 ; Rogers, 153 ; Nov. Marloth, 3229; Kolbe, 3615; Bulawayo, Kolbe; Chimanimani Mts. Johnson, 131?; Salisbury, Cecil, 260 ; Umgusa Riv. Baines; Umzingwani Riv. Baines; Odzani River Valley, Umtali, Teague, 272.

P. angolensis, Welw. var. albiflora, Engl.

Odzani River Valley, Umtali, Teague, 93.

P. grandiflora, Thunb.

Fl. Cap. V. i. 580 ; For. Fl. Cape, 296.

Sengwe Riv. Govt. Herb. 955.

P. madiensis, Oliv.

Fl. Trop. Afr. VI. i. 204.

Melsetter, 6000 ft. Sep. Swyn. 629 ; Chimanimani Mts. 7000 ft.

Oct. Swyn. 6097.

P. maschonica, Engl.

Hartley, Engler ; Umtali, Engler.

P. mellifera, Thunb.

Fl. Cap. V. i. 576 ; For. Fl. Cape, 296.

Bulawayo, Engler. 
P. Swynnertonii, S. Moore.

Journ. Linn. Soc. Bot. xl. 184.

Melsetter, $6000 \mathrm{ft}$. April, Swyn. 1411.

P. uhehensis, Engl.

Fl. Trop. Afr. VI. i. 202.

Chimanimani Mts. 4000 ft. Johnson, 131 ?; Chirinda, 3800 ft. Feb. Swyn. 190.

\section{P. spp.}

Mazoe, Jan. Eyles, 515, cf. P. abyssinica, Willd.

Gwanda, Noble, 74.

South Rhodesia, Rand, 394.

2036-Leucospermum saxosum, S. Moore.

Journ. Linn. Soc. Bot. xl. 185.

Chimanimani Mts. 7000 ft. Sep. Swyn. 652.

\section{SERIES SANTALALES.}

Family LXVII.-LORANTHACEAE, D. Don.

2074-Loranthus bulawayensis, Engl. (L. kraussianus, Gibbs.)

Fl. Trop. Afr. VI. i. 364.

Bulawayo, on Combretum sp. Marloth, 3378; Chubb, 400; Matopos, Gibbs, 274 ; Umvumvumvu Riv. 4000 ft. Oct. Swyn. 6001 ; Victoria, Monro, 717 ; Victoria Falls, Rogers, 5564.

L. Cecilae, N. E. Br.

Fl. Trop. Afr. VI. i. 373 ; Kew Bull. 1906, 168.

Bulawayo, Cecil, 96.

L. Dregei, Eckl. \& Zeyh.

Fl. Trop. Afr. VI. i. 311 ; Fl. Cap. ii. 575 ; Nat. Pl. 312.

Matopos, Engler; Sebakwe, 4000 ft. Oct. Eyles, 177.

var. subcuneifolius, Sprague.

Fl. Trop. Afr. VI. i. 312.

Matopos, Oct. Gibbs, 181.

L. erianthus, Sprague.

Fl. Trop. Afr. VI. i. 359.

Bulawayo, Chubb, 14a; Rogers, 5643; Sebakwe, Rogers; Victoria, Monro, 316, 827 ?

L. Eylesii, Sprague.

Fl. Trop. Afr. VI. i. 343 ; Kew Bull. 1911, 146.

Bulawayo, 4500 ft. Feb. Eyles, 1194 ; Victoria, Monro, 808.

L. guttatus, Sprague.

Fl. Trop. Afr. VI. i. 350; Kew Bull. 1911, 147.

Mazoe, 4700 ft. April, Eyles, 369. 
L. kalachariensis, Schinz. (L. curviflorus, Schinz.)

Fl. Trop. Afr. VI. i. 280.

Bulawayo, 4500 ft. May, on Acacia sp. Eyles, 78; Chubb, 313 ; Rand, 393; Rogers, 5644 ?; Matopos, Davy; Tuli Riv. Penther, 1626 ; Victoria Falls, July, Kolbe, 3184.

L. kraussianus, Meisn.

Fl. Cap. ii. 577 ; Nat. Pl. 76.

Mazoe, $4800 \mathrm{ft}$. April, Eyles, 301.

L. mweroensis, Baker.

Fl. Trop. Afr. VI. i. 304.

Salisbury, on Protea sp. Rand, 532.

L. namaquensis, Harv. (L. Meyeri, Presl.)

Fl. Trop. Afr. VI. i. 361 ; Fl. Cap. ii. 577 ; Cat. Afr. Pl. Welw. i. 932.

Victoria Falls, April, Flanagan, 3280 ; on Salix sp. Rogers, 5391. 7225, 5588 ; Bulawayo, Rogers, 5822.

L. quinquangulus, Engl. \& Schinz, var. pedicellatus, Sprague. (L. zambesicus, Gibbs.)

Fl. Trop. Afr. VI. i. 362 ; Journ. Linn. Soc. Bot. xxxvii. 467.

Victoria Falls, Allen, 138 ; fl. \& fr. Sep. Gibbs, 140.

L. Swynnertonii, Sprague.

Fl. Trop. Afr. VI. i. 390 ; Journ. Linn. Soc. Bot. xl. 188.

Chirinda Forest, 3700-4000 ft. on Vernonia podocoma, Sch. Bip.

Dec. Swyn. 141; also parasitic on peach-trees, Swyn.

L. virescens, N. E. Br.

Fl. Trop. Afr. VI. i. 309 ; Kew Bull. 1906, 168.

Salisbury, Rand, 1374, on Grewia sp. ; Cecil, 147.

L. spp.

Mazoe, March, Flanagan, 3022 ; Govt. Herb. 930.

Inyoka, Govt. Herb. 950.

Lomagundi, Govt. Herb. 989.

South Rhodesia, Rand, 222, 391.

2093-Viscum combreticola, Engl. (V. dichotomum, Don.)

Fl. Trop. Afr. VI. i. 404 ; Fl. Cap. ii. 581.

Mazoe, 5000 ft. fr. Nov. Eyles, 456 ; Harvest Valley, Rogers, 13577.

Y. matabelense, Engl.

Fl. Trop. Afr. VI. i. 410.

Matopos, $5000 \mathrm{ft}$. Engler, $2846 a$.

Y. Menyharthii, Engl. \& Schinz.

Fl. Trop. Afr. VI. i. 410.

Matopos, on Croton gratissimus, Burch. Gibbs, 16. 
Genus No.

Y. tuberculatum, A. Rich.

Fl. Trop. Afr. VI. i. 396.

Matopos, Engler.

У. yerrucosum, Harv.

Fl. Trop. Afr. VI. i. 408 ; Fl. Cap. ii. 581.

Matopos, Galpin, 7087; fl. \& fr. Sep. Gibbs, 65 ; Bulawayo, 4500 ft. Dec. on Acacia sp. Eyles, 1139 ; Rogers, 5642.

Y. sp.

South Rhodesia, Rand, 393.

Family LXIX.-SANTALACEAE, $R . B r$.

2104-Colpoon compressum, Berg.

For. Fl. Cape, 303.

Matopos, Oct. Gibbs, 183 ; Engler; Govt. Herb. 891; Lomagundi, Govt. Herb. 974.

2108-Osyris abyssinica, Hochst.

Fl. Trop. Afr. VI. i. 433 ; Cat. Afr. Pl. Welw. i. 938.

Melsetter, $6000 \mathrm{ft}$. Sep. Swyn. 622; North Melsetter, 50006000 ft. fr. Oct. Swyn. 6102 ; Victoria, Monro, 643 ; Bulawayo, Rogers, 13677*.

2118-Thesium brevibarbatum, Pilger.

Fl. Trop. Afr. VI. i. 416.

Umtali, 3900 ft. Sep. Engler, 3176.

T. goetzeanum, Engl.

Fl. Trop. Afr. VI. i. 418.

Between Hartley \& Gadzema, 5000 ft. Engler, 3013 ; Matopos, 5000 ft. Nov. Eyles, 1146 ; Salisbury, Marshall.

T. gracile, $A$. W. Hill.

Fl. Trop. Afr. VI. i. 419 ; Kew Bull. 1910, 185.

Sebakwe, 4000 ft. Dec. Eyles, 85.

T. multiramulosum, Pilger.

Fl. Trop. Afr. VI. i. 419.

Umtali, 3900 ft. Engler, 3138, 3146.

T. rhodesiacum, Pilger.

North of Hartley, Engler.

T. Rogersii, A. W. Hill.

Fl. Trop. Afr. VI. i. 1059; Kew Bull. 1913, 78.

Victoria Falls, on island, Rogers, 5467.

T. scabridulum, A. W. Hill.

Fl. Trop. Afr. VI. i. 424 ; Journ. Linn. Soc. Bot. xl. 189.

Melsetter, 6000 ft. fl. \& fr. Sep. Swyn. 2124. 
Genus No.

T. sp.

South Rhodesia, Rand, 206.

Family LXXI.-OPILIACEAE, Valeton.

2122-0pilia amentacea, Roxb.

Fl. Trop. Afr. i. 352 ; For. Fl. Port. E. Afr. 30 ; Cat. Afr. Pl. Welw. i. 142.

Sabi Riv, 1000 ft. Nov. Swyn. 1204.

FAmily LXXII.-OLACACEAE, Lindl.

2131-Olax dissitiflora, Oliv.

Fl. Trop. Afr. i. 350 ; For. Fl. Port. E. Afr. 30.

Matopos, Oct. Gibbs, 263; Victoria Falls, Gibbs ; Rogers, 7462 ;

Victoria Monro, 560.

2136-Ximenia americana, $L$.

Fl. Trop. Afr. i. 346 ; For. Fl. Port. E. Afr. 30.

Victoria Falls, Sep. Galpin, 7060 ; Rogers, 5997 ; Chirinda, 3500 ft. Oct. Swyn. 175; Bulawayo, Monro, 64 ; Chubb, 332, 354 ; Rand, May, 423, cf. var. microphylla, Welw.

X. caffra, Sond.

Fl. Cap. i. 235 ; For. Fl. Port. E. Afr. 30.

Victoria Falls, Sep. Gibbs, 130 ; Allen, 159 ; Rogers, 5468, 5985;

Matopos, Engler; Gibbs; Gwanda, Noble, 46; Victoria, Monro, 640 ; Bulawayo, Monro, 37, 82.

\section{X. spp.}

Bulawayo, Monro, 1 ; Chubb, 340.

Umtali, Swyn. 6619, cf. X. americana, L.

\section{Series ARISTOLOCHIALES.}

Family LXXV.-RAFFLesiaceae, Dumort.

2179-Pilostyles æthiopica, Welw.

Fl. Trop. Afr. VI. i. 131 ; Cat. Afr. Pl. Welw. i. 908.

Victoria, Monro, 962, 457, on Brachystegia sp.

Family LXXVI.-HYDNORACEAE, Solms-Laub.

2182-Hydnora africana, Thunb.

Fl. Cap. V. i. 486 ; Cat. Afr. Pl. Welw. i. 910.

Bulawayo, Monro. 


\section{SERIES POLYGONALES.}

Family LXXVII.-POLYGONACEAE, Lindl.

Genus No

2195-Rumex abyssinicus, Jacq.

Fl. Trop. Afr. VI. i. 114 ; Cat. Afr. Pl. Welw. i. 905.

Melsetter, 6000 ft. April, Swyn. 1506.

R. nepalensis, Spreng.

Fl. Trop. Afr. VI. i. 117 ; Fl. Cap. V. i. 473.

Salisbury, Rogers, 4085.

2201-Polygonum acuminatum, H. B. \& $K$.

Fl. Trop. Afr. VI. i. 112.

Upper Buzi, 3000 ft. June, Swyn. 356; Chirinda Forest, 3700-4000 ft. Dec. Swyn. 1512.

P. aviculare, $L$.

Fl. Trop. Afr. VI. i. 105 ; Fl. Cap. V. i. 464.

Bulawayo, 4500 ft. Aug. Eyles, 1245 ; Rogers, 5518, 13622 ; Chubb, 35 ; Victoria, Monro, 85.

P. barbatum, $L$.

Fl. Trop. Afr. VI. i. 109 ; Fl. Cap. V. i. 467.

Victoria Falls, Engler; Salisbury, Rogers, 4083 ?; Bulawayo, Rogers, 13620.

P. plebeium, R. Br. (P. herniarioides, Del.)

Fl. Trop. Afr. VI. i. 105.

Matopos, Sep. Gibbs, 63 ; Gwelo, Jan. Gardner, 44.

P. serrulatum, Lag.

Fl. Trop. Afr. VI. i. 107 ; Fl. Cap. V. i. 467.

Victoria Falls, Rogers, 5030, 5575, 5578, 6016; Salisbury, Rogers, 4084; Odzani River Valley, Umtali, Teague, 183.

P. tomentosum, Willd.

Fl. Trop. Afr. VI. i. 110 ; Fl. Cap. V. i. 468 ; Cat. Afr. Pl. Welw. i. 905 .

Matopos, March, Flanagan, 2959 ; Rogers, 5647; Victoria Falls, Rogers, 5625, 7176; Salisbury, Marshall.

P. sp.

South Rhodesia, Rand, 223.

2204-0xygonum alatum, Burch.

Fl. Trop. Afr. VI. i. 99 ; Fl. Cap. V. i. 460 ; Cat. Afr. Pl. Welw. i. 902.

Victoria Falls, Allen, 287.

0. dregeanum, Meisn.

Fl. Cap. V. i. 461.

Redbank, April, Flanagan, 3183. 
A Record of Plants Collected in Southern Rhodesia.

Genus No. pubescens, C. H. Wright.

Fl. Trop. Afr. VI. i. 101; Kew Bull. 1909, 187.

Between Salisbury and Headlands, Cecil, 155; between Salisbury and Umtali, Cecil, 44 ; Umtali, Cecil, $235 a$.

0. Zeyheri, Sond.

Fl. Cap. V. i. 460.

Bulawayo, Jan. Gardner, 61.

0. spp.

Victoria Falls, Rogers, 7198, probably n. sp.

Between Bulawayo and Plumtree, April, Flanagan, 3182.

\section{SERIES CENTROSPERIMAE.}

FAmily LXXVIII.-CHENOPODIACEAE, Less.

\section{3-Chenopodium Botrys, $L$.}

Fl. Trop. Afr. VI. i. 79 ; Fl. Cap. V. i. 439.

Matopos, Rogers; Odzani River Valley, Umtali, Teague, 199.

C. schraderianum, Roem. \& Schultes. (C. foetidum, Schrad.; C. graveolens, Lag. \& Rodr.)

Fl. Trop. Afr. VI. i. 80 ; Fl. Cap. V. i. 439 ; Cat. Afr. Pl. Welw.

i. 898 .

Melsetter, 6000 ft. April, Swyn. 1507.

C. sp.

Mazoe, 4700 ft. Aug. Eyles, 180.

2240-Kochia decumbens (Hochst.), Torre \& Harms. (Pentodon decumbens, Hochst.)

Victoria, Monro, 925.

\section{FAMily LXXIX.--AMARANTACEAE, Juss.}

2292-Celosia trigyna, $L$.

Fl. Trop. Afr. VI. i. 19 ; Fl. Cap. V. i. 404 ; Cat. Afr. Pl. Welw. i. 884 .

Matopos, March, Flanagan, 2941, 2951 ; Victoria Falls, April, Flanagan, 3294 ; Rogers, 7141; May, Eyles, 143 ; between Wankie and Victoria Falls, Rogers, 5272; Chipete Forest, $3800 \mathrm{ft}$. April, May, Swyn. 507 ; Upper Buzi, $3000 \mathrm{ft}$. Swyn. 1514 ; Matabeleland, Holub.

C. sp.

South Rhodesia, Rand, 201. 
Genus No.

2293-Hermbstædtia elegans, Moq.

Fl. Trop, Afr. VI. i. 26 ; Fl. Cap. V. i. 407.

Bulawayo, Rogers, 5928.

2299-Amarantus græcizans, L. (A. Thunbergii, Moq.)

Fl. Trop. Afr. VI. i. 34 ; Fl. Cap. V. i. 411 ; Cat. Afr. Pl. Welw. i. 887.

Matopos, Rogers, 5247; Gwelo, Sr. Phil. 6; Chirinda, 3800 ft. May, Swyn. 463 ; Salisbury, Govt. Herb. 605.

A. viridis, $L$.

Fl. Trop. Afr. VI. i. 33 ; Fl. Cap. V. i. 411 ; Cat. Afr. Pl. Welw. i. 888 .

Victoria Falls, Rogers, 7066.

2309-Cyphocarpa angustifolia, Lopr. (Sericocoma angustifolia, Hook.f.)

Fl. Trop. Afr. VI. i. 53 ; Fl. Cap. V. i. 415 ; Cat. Afr. Pl. Welw. i. 889.

Bulawayo, 4500 ft. Dec. Eyles; Gardner, 87 ; Victoria Falls, April, Flanagan, 3204 ; Victoria, Monro, 927.

2312-Cyathula cylindrica, Moq.

Fl. Trop. Afr. VI. i. 46 ; Fl. Cap. V. i. 420.

Mazoe, 5000 ft. March, Eyles, 249.

C. prostrata, Blume.

Fl. Trop. Afr. VI. i. 43.

Chirinda Forest, 3700-4000 ft. April, May, Swyn. 269a, 340.

2317-Erya lanata, Juss.

Fl. Trop. Afr. VI. i. 39 ; Fl. Cap. V. i. 426.

Bulawayo, $4500 \mathrm{ft}$. Eyles, 83.

E. leucura, Moq.

Fl. Trop. Afr. VI. i. 39 ; Fl. Cap. V. i. 426.

Mazoe, 4500 ft. March, Eyles, 276; Matopos, March, Flanagan, 2952 ; Rogers, 5165 ; Plumtree, April, Flanagan, 3208 ; Salisbury, May, Flanagan, 3271; Victoria, Monro, 372; Odzani River Valley, Umtali, Teague, 107.

\section{E. sp.}

South Rhodesia, Rand, 203.

2324-Psilotrichum gracilentum, C. B. Cl.

Fl. Trop. Afr. VI. i. 59.

Umvumvumvu Riv. 4000 ft. April, Swyn. 777 ; North Melsetter, 5000-6000 ft. Oct. Swyn. 6640.

2328-Achyranthes aspera, $L$.

Fl. Trop. Afr. VI. i. 63 ; Fl. Cap. V. i. 428 ; Cat. Afr. Pl. Welw. i. 893.

Bulawayo, 4700 ft. Feb. Eyles, 105 ; Victoria Falls, fl. \& fr. Sep. Gibbs, 171 ; Engler ; Rogers, 5056 ; Mazoe, March, Eyles, 299. 
A Record of Plants Collectcd in Southern Rhodesia.

Genus No.

var. argentea, $C . B . C l$. (A. argentea, Lam.)

Fl. Trop. Afr. VI. i. 63.

Mazoe, 4800 ft. March, Eyles, 298 ; Odzani River Valley, Umtali, Teague, 81.

var. pinguispicata, C. B. Cl.

Matabeleland, Oates.

A. bidentata, Blume.

Fl. Trop. Afr. VI. i. 64 ; Cat. Afr. Pl. Welw. i. 894.

Chirinda Forest, 3700-4000 ft. June, Swyn. 1510.

A. leptostachya, E. Mey. (Achyropsis leptostachya, Hook. f.)

Fl. Trop. Afr. VI. i. 66 ; Fl. Cap. V. i. 430.

Shashi Riv. Holub.

A. sp.

Mazoe, 5200 ft. Jan. Eyles, 520.

2335-Alternanthera echinata, Smith. (A. achyrantha, R. Br.)

Fl. Trop. Afr. VI. i. 74; Fl. Cap. V. i. 432 ; Cat. Afr. Pl. Welw. i. 896. Victoria Falls, Rogers, 7193 ; Bulawayo, Rogers, 13621 ; Hartley, Mundy.

A. nodiflora, $R . B r$.

Fl. Trop. Afr. VI. i. 73.

Victoria, Monro, 944 ; Salisbury, Rogers.

A. sessilis, $R$. Br.

Fl. Cap. V. i. 432.

Victoria Falls, Sep. Gibbs, 121.

FAMILY LXXX.-NYCTAGINACEAE, Lindl.

2349-Boerhavia pentandra, Burch. (B. grandiflora, A. Rich.)

Fl. Trop. Afr. VI. i. 7 ; Fl. Cap. V. i. 396.

Bulawayo, 4500 ft. Dec. Eyles, 19 ; Aug. 1228 ; Gwelo, Sr. Phil. 56 ; Victoria Falls, Rogers, 7419 ?

B. plumbaginea, Cav. (B. dichotoma, Vahl.)

Fl. Trop. Afr. VI. i. 7 ; Cat. Afr. Pl. Welw. i. 883.

Gwelo, Gardner, 20 ; Mazoe, 5100 ft. April, Eyles, 333.

B. repens, $L$. var. diffusa, Hook. $f$.

Fl. Trop. Afr. VI. i. 5 ; Fl. Cap. V. i. 395.

Victoria Falls, Rogers, 5628 ?

Family LXXXIII.-PHYTOLACCACEAE, Lindl.

2376-Limeum fenestrata (Fenzl.), Torre \& Harms. (Semonvillea fenestrata, Fenzl.)

Fl. Cap. i. 152.

North of Bulawayo, $3400 \mathrm{ft}$. Dec. Eyles, 1133; Victoria Falls, April, Flanagan, 3307 ; Rogers, 5607, 7264, 7436, 7207. 
Genus No.

L. viscosum, Fenzl.

Fl. Trop. Afr. ii. 595 ; Cat. Afr. Pl. Welw. i. 421.

Shashi Riv. Jan. Rand, 57 ; Salisbury, March, Flanagan, 3006.

2380-Phytolacca dodecandra, L'Hérit. (P. abyssinica, Hoffm.)

Fl. Trop. Afr. VI. i. 97 ; Fl. Cap. i. 157, \& V. i. 457 ; Nat. Pl. 263 ; Cat. Afr. Pl. Welw. i. 901.

Salisbury, Engler; Lusitu Riv. 3000 ft. fl. \& fr. Sep. Oct. Swyn. 2118 ; Victoria Falls, Rogers, 7255*.

\section{P. sp.}

Salisbury, March, Flanagan, 2998.

2382-Giesekia pharnaceoides, $L$.

Fl. Trop. Afr. ii. 593 ; Cat. Afr. Pl. Welw. i. 419,

Shashi Riv. Jan. Rand. 56 ; Gwai, April, Flanagan, 3289 ; Victoria Falls, April, Flanagan, 3242 ; Rogers, 7263, 7005 ; Victoria, Monro, 881.

G. spp.

Bulawayo, Jan. Gardner, 4 ; Malindi, Jan. Allen, 235.

Family LXXXIV.-AIZOACEAE, $A$. $B r$.

2387-Mollugo Cerviana, Seringe.

Fl. Trop. Afr. ii. 591 ; Fl. Cap. i. 138; Cat. Afr. Pl. Welw. i. 417.

Salisbury, Rogers.

M. hirta, Thunb. var. virens, Fenzl. (M. Glinus, A. Rich.; Glinus lotoides, L.)

Fl. Trop. Afr. ii. 590 ; Fl. Cap. i. 137 ; Cat. Afr. Pl. Welw. i. 416. Bulawayo, May, Rand, 331.

M. nudicaulis, Lam.

Fl. Trop. Afr. ii. 591 ; Cat. Afr. Pl. Welw. i. 417.

Victoria Falls, Rogers, 5701.

2388-Glinus sp.

Victoria Falls, Allen, 284, cf. G. Bainesii, Pax.

2389-Pharnaceum xerrucosum, E.\&Z. (P. salsoloides, Burch; Hypertelis verrucosa, Fenzl.)

Fl. Trop. Afr. ii. 592 ; Fl. Cap. i. 144 ; Cat. Afr. Pl. Welw. i. 418.

Victoria, Monro, 850 ; Salisbury, Rogers, 5520.

P. Zeyheri, Sond.

Fl. Cap. i. 141.

Matopos, Sep. Oct. Gibbs, 45 ; Engler.

2393-Orygia decumbens, Forsk.

Fl. Trop. Afr. ii. 589; Fl. Cap. i. 136 ; Cat. Afr. Pl. Welw. i. 415. Bulawayo, $4500 \mathrm{ft}$. Dec. Eyles, 30. 
A Record of Plants Collected in Southern Rhodesia.

Genus No.

2405-Mesembrianthemum Mahoni, N. E. Br.

Gard. Chron. 1902, ii. 190.

Odzani River Valley, Umtali, Teague.

M. sp.

Matopos, Nov. Eyles, 1190.

FAmily LXXXV.-PORTULACACEAE, Reichb.

2406-Talinum caffrum, E. \& Z. (Portulaca caffra, Thunb.)

Fl. Trop. Afr. i. 150 ; Fl. Cap. ii. 385 ; Nat. Pl. 593 ; Cat. Afr.

Pl. Welw. i. 54.

Bulawayo, Dec. Gardner, 67 ; Rogers, 5913; Victoria Falls, Rogers, 5623.

2412-Anacampseros rhodesica, N. E. Br.

Kew Bull. 1914, 132.

Matopos, J. G. McDonald \& W. E. Dowsett; Salisbury, Mundy.

FAmily LXXXVII.-CARYOPHYLLACEAE, Reichb.

2455-Polycarpæa corymbosa, Lam. (Polia arenaria, Lour.)

Fl. Trop. Afr. i. 145 ; Fl. Cap. i. 133 ; Cat. Afr. Pl. Welw. i. 51 .

Bulawayo, May, Rand, 350 ; Victoria Falls, April, Flanagan, 3030 ; Rogers, 7028; Mazoe, 4400 ft. April, Eyles, 310; Victoria, Monro, 952 ; Chimanimani Mts. 7000 ft. Sep. Swyn. 1804.

2467-Pollichia campestris, Ait.

Fl. Trop. Afr. VI. i. 10 ; Fl. Cap. i. 133, \& V. i. 399.

Victoria Falls, on islands, Sep. Gibbs, 118; Engler.

2469-Corrigiola drymarioides, Bak. $f$.

Journ. Linn. Soc. Bot. xl. 181.

Chimanimani Mts. 7000 ft. fl. \& fr. Sep. Swyn. 2159.

C. litoralis, $L$.

Fl. Trop. Afr. VI. i. 12 ; Fl. Cap. i. 132, and V. i. 401.

Bulawayo, 4500 ft. Aug. Eyles, 1241 ; Macheke, Engler; Victoria, Monro, 364 ; Salisbury, Rogers.

2490-Silene Burchellii, Otth.

Fl. Trop. Afr. i. 139 ; Fl. Cap. i. 128 ; Cat. Afr. Pl. Welw. i. 49.

Gwelo, Jan. Gardner, 23 ; Salisbury, Engler ; Rand, 1389 ; Mt.

Pene, 7000 ft. Oct. Swyn. 6069 ; Harvest Valley, Rogers. 


\section{SERIES RANALES.}

Family LXXXVIII.-NYMPHAEACEAE, $D C$.

Genus No. phæa lotus, $L$.

Fl. Trop. Afr. i. 52 ; Cat. Afr. Pl. Welw. i. 22.

North of Bulawayo, Engler.

N. stellata, Willd. (N. malabarica, Poir.)

Fl. Trop. Afr. i. 52 ; Fl. Cap. i. 14 ; Nat. Pl. 33 ; Cat. Afr. Pl. Welw. i. 22 ; also see note in Kew Bull. 1906, 183.

Matopos, Marloth ; 4600 ft. April, Eyles, 35 ; Rogers ; Bulawayo, Engler; Salisbury, May, Flanagan, 3248; South Rhodesia, Rand, 16.

\section{FAMiLy XCI.-RANUNCULACEAE, Juss.}

2541-Anemone peneensis, E. G. Baker.

Journ. Linn. Soc. Bot. xl. 16.

Mt. Pene, 6500 ft. Sep. Swyn. 783.

2542-Clematis brachiata, Thunb.

Fl. Cap. i. 2.

Bulawayo, Chubb, 19.

C. Kirkii, Oliv.

Fl. Trop. Afr. i. 5.

Salisbury, Darling, in Herb. Bolus, 10797; between Bulawayo and Victoria Falls, Davy; Mazoe, May, Flanagan, 3200 ; Chirinda, Swyn. ; Odzani River Valley, Umtali, Teague, 23.

C. orientalis, $L$.

Cat. Afr. Pl. Welw. i. 3.

Victoria, Monro, 1002.

C. simensis, Fres. (C. orientalis, L. var. simensis, O. Kuntze.)

Fl. Trop. Afr. i. 6 ; Cat. Afr. Pl. Welw. i. 3.

Chirinda, Swyn.

S. Stanleyi, Hook. (C. villosa, DC.)

Fl. Trop. Afr. i. 6 ; Fl. Cap. i. 2 ; Cat. Afr. Pl. Welw. i. 2.

Salisbury, Dec. Rand, 1 and 436 ; Bulawayo, 4500 ft. Eyles, 1121 ;

Monro, 108 ; Matopos, 4500 ft. Feb. Eyles, 1172 ; Rogers, 5266 ;

Umtali, Engler; Chirinda, Swyn. ; South Rhodesia, Rand, 289.

C. Thunbergii, Steud. (C. orientalis, L. var. Thunbergii, O. Kuntze.)

Fl. Trop. Afr. i. 6 ; Fl. Cap. i. 2 ; Cat. Afr. Pl. Welw. i. 3.

Victoria Falls, April, Flanagan, 3244, 3238; Mazoe, $4300 \mathrm{ft}$. April, Eyles, 326 ; Victoria, Monro, 1002 ; Odzani River Valley, Umtali, Teague, 210. 
Genus No. C. virona, $L$.

South Rhodesia, Rand, 288.

C. wightiana, Wall.

Chirinda, Swyn.

C. spp.

Gwelo, Jan. Gardner, 9, cf. C. Kirkii, Oliv.

Mazoe, 4800-5000 ft. March, Eyles, 281, probably n. sp.

Salisbury, May, Flanagan, 3199 (same as Eyles 281).

Victoria Falls, Rogers, 5077.

2546-Ranunculus pinnatus, Poir. (R. pubescens, Thunb.)

Fl. Trop. Afr. i. 9; Fl. Cap. i. 6 ; Cat. Afr. Pl. Welw. i. 4.

Salisbury, Dec. Rand, 79 ; July, Rand, 435; Bulawayo, May, Rand, 287; Gwelo, Jan. Gardner, 25; Mazoe, 4500 ft. Nov. Eyles, 450.

R. plebeius, $R$. $B r$.

Fl. Cap. i. 6.

Chirinda Forest, 3700-4000 ft. Swyn. 345.

2548-Thalictrum rhynchocarpum, Dill. \& Rich.

Fl. Trop. Afr. i. 8.

Chipete and Chirinda, Swyn.

Family XCIV.-MENISPERMACEAE, $D C$.

2570-Cocculus villosus, DC. (Cebatha hirsuta, O. Kuntze.)

Fl. Trop. Afr. i. 45 ; Cat. Afr. Pl. Welw. i. 18.

Sabi Riv. 1000 ft. fr. Nov. Swyn. 1756a.

2574-Cissampelos Pareira, $L$.

Fl. Trop. Afr. i. 45 ; Fl. Cap. i. 11 ; Cat. Afr. Pl. Welw. i. 18.

North of Hartley, Engler ; Victoria Falls, Rogers, 5616.

var. mucronata, Engl. (C. mucronata, A. Rich.)

Matopos, Oct. Gibbs, 243 ; Chipete Forest, 3800 ft. Jan. Swyn.

220 ; Chirinda, Swyn.

C. torulosa, E. Mey.

Fl. Trop. Afr. i. 46 ; Fl. Cap. i. 11.

Chipete Forest, 3800 ft. Jan. Swyn. 218, 219.

2577-Tiliacora funifera, Oliv.

Fl. Trop. Afr. i. 44.

Victoria Falls, Rain Forest, male, Sep. Gibbs, 302 ; Kirk; Chirinda, Swyn.

2594-Dioscoreophyllum chirindense, Swyn.

Journ. Linn. Soc. Bot. xl. 19.

Chirinda Forest, 3700-4000 ft. fl. Jan. and March, Swyn. 100, 100a, fr. Dec. Swyn. 6521. 
FAmily XCVIII.-ANONACEAE, L. C. Rich.

Genus No.

2673-Uvaria gazensis, Swyn. \& E. G. Baker.

Journ. Linn. Soc. Bot. xl. 17.

Chirinda, 3700-4000 ft. Oct. Swyn. 1326.

2691-Popowia obovata, Engl. \& Diels.

Victoria Falls, Allen, 144 ; Rogers, 7081, 5540, 5618; Sebawke. 4000 ft. Dec. Eyles, 162 ; Chikore, 3000 ft. April, Swyn. 187.

2716-Hexalobus senegalensis, A. DC.

Fl. Trop. Afr. i. 27.

Matopos, Oct. Gibbs, 217; Sep. Galpin, 7086.

2724 - Artabotrys brachypetala, Benth.

Fl. Trop. Afr. i. 28; For. Fl. Port. E. Afr. 8.

Matopos, Oct. Gibbs, 252 ; Victoria, Monro, 795.

A. Monteiroae, Oliv.

Chimanimani Mts. 7000 ft. Sep. Swyn. 1764.

2729-Anona senegalensis, Pers.

Fl. Trop. Afr. i. 16 ; Fl. Cap. ii. 583 ; Cat. Afr. Pl. Welw. i. 8 ; For. Fl. Port. E. Afr. 7.

Matopos, Davy ; Oct. Gibbs, 256 ; Gwai Forest, 3600 ft. Allen, 240 ; Hartley, Engler; Salisbury, Rand, 1342; Victoria, Monro, 305, 565 ; Chirinda, 3500 ft. Swyn. 191.

var. rhodesiaca, Engl. \& Diels.

Salisbury, Sep. Engler, 3080 ; Matopos, Nov. Marloth, 3376.

Family CI.-MONimiaCEAE, Dumort.

$2759 a$-Xymalos monospora, Baill.

Fl. Trop. Afr. VI. i. 169 ; Fl. Cap. V. i. 493 ; For. Fl. Cape, 288.

Chirinda Forest, 3700-4000 ft. June, 663; Chimanimani Mts. 7000 ft. Sep. Swyn. 1112 ; Chipete Forest, Swyn.

\section{Family CII.-LAURACEAE, Lindl.}

2825-Cassytha ciliolata, Nees. (C. capensis, Meisn.)

Fl. Cap. V. i. 501.

Victoria Falls, April, Flanagan, 3275.

C. filiformis, $L$.

Fl. Trop. Afr. VI. i. 188; Fl. Cap. V. i. 500 ; Cat. Afr. Pl. Welw. i. 915 .

Victoria Falls, Rogers, 5596. 
FAmily CIII.-HERNANDIACEAE, Dumort.

Genus No. 2830-Gyrocarpus sp.

Victoria Falls, Allen, 401. This "may possibly belong to $G$. asiaticus, Willd. The fruits, however, differ in being glabrous." Fl. Trop. Afr. VI. i. 190.

\section{SERIES RHOEADALES.}

FAmily CIV.-PAPAVERACEAE, B. Juss.

2852-Argemone mexicana, $L$.

Fl. Trop. Afr. i. 54 ; Cat. Afr. Pl. Welw. i. 23.

Bulawayo, Rogers.

Family CV.-CRUCIFERAE, B. Juss.

2875-Heliophila sp.

South Rhodesia, Rand, 96.

2949-Brassica spp.

Mazoe, 4800 ft. March, Eyles, 295 ; Bulawayo, Rogers, 5756 ; Salisbury, Rogers, 5770.

2965--Nasturtium fluxiatile, E. Mey.

Fl. Cap. i. 21 ; Harv. Gen. S.A. Pl. 7.

Bulawayo, Jan. Rand, 21.

\section{FAmILy CVII.-CAPPARIDACEAE Lindl.}

3082-Cleome hirta, Oliv.

Fl. Trop. Afr. i. 81; Cat. Afr. Pl. Welw. i. 28.

Victoria Falls, Allen, 108 ; Rogers, 7257, 6007 ; Bulawayo, Jan.

Gardner, 59 ; Matopos, Rogers, 5151, 5161a.

C. maculata, Szyszyl. (Tetratelia maculata, Sond.)

Fl. Cap. i. 58.

Shashi Riv. Jan. Rand, 22.

C. monophylla, $L$.

Fl. Trop. Afr. i. 76 ; Fl. Cap. i. 156.

Bulawayo, Dec. Rand, 73 ; Chubb, $13 a$; Dec. Eyles, 8; Rogers, 5757 ; Matopos, Flanagan, 2986; Redbank, April, Flanagan, 3185 ; Victoria, Monro, 817 ; Victoria Falls, Rogers, 5579. 
Genus No.

3087-Gynandropsis pentaphylla, DC. (Pedicellaria pentaphylla, Schrank.)

Fl. Trop. Afr. i. 82 ; Fl. Cap. i. 55.

Bulawayo, 4500 ft. Dec. Eyles, 1126 ; Jan. Rand, 23 ; Rogers, 5912 ; Victoria Falls, April, Flanagan, 3206; Chimanimani Mts. Swyn.

3101-Capparis tomentosa, Lam.

Fl. Trop. Afr. i. 96; Cat. Afr. Pl. Welw. i. 31.

Victoria Falls, fl. \& fr. Sep. Gibbs, 138; Allen, 170 ; Sebakwe, 4000 ft. Oct. Eyles, 173 ; Victoria, Monro, 318, 563, 829, 521 ; Bulawayo, Chubb, 342.

C. spp.

Victoria, Monro, 448, 432.

3108-Courbonia decumbens, Brongn.

Fl. Trop. Afr. i. 88; For. Fl. Port. E. Afr. 10.

Sabi Riv. 1000 ft. fr. Nov. Swyn. 1207, 1384.

3109 -Cadaba juncea, Hook. (Schepperia juncea, DC.)

Fl. Cap. i. 59 ; For. Fl. Cape, 121.

Bulawayo, Chubb, 347.

C. natalensis, Sond.

Fl. Cap. i. 59 ; Nat. Pl. 261 ; For. Fl. Cape, 121.

Bulawayo, 4500 ft. Oct. Eyles, 1087 ; Monro, 101.

3112-Mærua arenicola, Gilg .

Victoria, Monro, 789.

M. caffra (Bernh.), Pax. (Niebuhria triphylla, Wendl.)

Bulawayo, Engler.

M. maschonica, Gilg.

Umtali, Engler.

M. neryosa, Oliv. (Niebuhria nervosa, Hochst.)

Fl. Trop. Afr. i. 84 ; Nat. Pl. 260; For. Fl. Port. E. Afr. 9; Fl. Cap. i. 60.

Matopos, Galpin, 6967 ; Sabi Riv. Swyn.; Victoria, Monro, 302, 432, 448; Bulawayo, Monro, 85, 86.

var. flagellaris, Oliv.

Fl. Trop. Afr. i. 84.

Bulawayo, Rand, Sep. 576 ; Matopos, Oct. Gibbs, 205 ; Victoria, Monro, 364.

M. sp.

Victoria, Monro, 318.

3113--Thylachium verrucosum, Klotzsch. (T. africanum, Lour.)

Fl. Trop. Afr. i. 82 ; For. Fl. Port. E. Afr. 9.

Sabi Riv. 1000 ft. fr. Nov. Swyn. 1206. 


\section{SERIES SARRACENIALES.}

Family CXII.-DROSERACEAE, DC.

Genus No.

3136-Drosera burkeana, Planch.

Fl. Cap. i. 76; Fl. Trop. Afr. ii. 402.

Victoria Falls, Rogers*.

D. indica, $L$.

Fl. Trop. Afr. ii. 402.

Victoria, Monro, 1032.

D. ramentacea, Burch.

Fl. Trop. Afr. ii. 403 ; Fl. Cap. i. 77.

Matopos, $5000 \mathrm{ft}$. April, Eyles, 53.

D. sp.

Matopos, 4500 ft. Nov. Eyles, 1099.

\section{Series ROSALES.}

Family CXIII.-POdostemonaCEAE, L. C. Rich.

3140-Tristicha alternifolia, Tul.

Victoria Falls, fl. \& young fr. Sep. Gibbs, 321 ; Engler.

T. trifaria, Tul.

Fl. Trop. Afr. VI. i. 121.

Victoria Falls, Livingstone Island, Sep. Gibbs, 320.

3151-Dicræa tenax, C. H. Wright.

Fl. Trop. Afr. VI. i. 121; Kew Bull. 1909, 213.

Victoria Falls, Livingstone Island, July, Kolbe, 3149.

3159-Sphærothylax sp.

Victoria Falls, fr. Sep. Gibbs, 322.

FAmily CXIV.-HYDROSTACHYACEAE, Warm.

3160-Hydrostachys polymorpha, Klotzsch.

Fl. Trop. Afr. VI. i. 130.

North Melsetter, 4000 ft. Swyn. 818.

Family CXV.-CRASSULACEAE, DC.

3166-Kalanchoe crenata, Haw. (K. egyptiaca, DC.)

Fl. Trop. Afr. ii. 394 ; Fl. Cap. ii. 379 ; Cat. Afr. Pl. Welw. i. 328.

Victoria Falls, Rogers, $5000 a$. 
Genus No

K. glandulosa, Hochst.

Fl. Trop. Afr. ii. 396 ; Cat. Afr. Pl. Welw. i. 328.

Victoria Falls, Rogers, 7443.

var. rhodesica, Baker $f$.

Journ. Bot. 1899, 434.

Salisbury, July, Rand, 465 ; Engler; Umtali, Engler; Gwelo, Sr. Phil. 8 and 14.

K. paniculata, Harv.

Fl. Cap. ii. 380.

Bulawayo, May, Rand, 321; Victoria Falls, Rogers, 7444.

K. pilosa, Baker.

Victoria Falls, Rogers, 5235, 5058, 7213.

K. rotundifolia, Haw.

Fl. Cap. ii. 379 ; Nat. Pl. 94.

Bulawayo, May, Rand, 319 ; Matopos, Rogers, 5265 ; Victoria, Monro, 1010; Odzani River Valley, Umtali, Teague, 254.

K. thyrsiflora, Harv.

Fl. Cap. ii. 380.

Bulawayo, Rogers, 13723*.

K. spp.

Matopos, April, Flanagan, 3172, cf. K. glandulosa, Hochst.

Victoria Falls, Rogers, 5236; South Rhodesia, Rand, 320.

3168-Crassula campestris, E. \& Z. (Tillea pharnaceoides, Hochst.)

Fl. Trop. Afr. ii. 387 ; Fl. Cap. ii. 351.

Matopos, 4500-5000 ft. March, Eyles, 1027.

C. nivalis, $E$. \& $Z$.

Fl. Cap. ii. 356.

Victoria Falls, Galpin, 7369.

C. sarcolipes, Harr.

Fl. Cap. ii. 355.

Victoria, Monro, 917.

C. subulata, Hook. (Tillaa subulata, Benth. \& Hook. f.)

Fl. Trop. Afr. ii. 387 ; Fl. Cap. ii. 352 ; Cat. Afr. Pl. Welw. i. 325.

Victoria, Monro, 1072.

FAmily CXVII.-SAXIFRAGACEAE, DC.

3201 - Yahlia capensis, Thunb.

Fl. Trop. Afr. ii. 384 ; Fl. Cap. ii. 306 ; Cat. Afr. Pl. Welw. i. 324 ; Harv. Gen. S.A. Pl. 98.

Bulawayo, May, Rand, 334 ; Eyles; Jan. Gardner, 83 ; Victoria Falls, Allen, 119; Matopos, Oct. Gibbs, 308; March, Flanagan, 2962 ; Engler; Matabeleland, Oates. 
Genus No.

var. linearis, E. Mey.

Bulawayo, Dec. Rand, 63 ; Rogers, 5484.

Y. oldenlandioides, Roxb.

Fl. Trop. Afr. ii. 384.

Between Wankie and Victoria Falls, Rogers, 5271.

3241 -Choristylis shirensis, Baker $f$.

Melsetter, 6000 ft. Sep. Swyn. 607 ; Chirinda, 3800 ft. Swyn.

202, 203 ; Mt. Pene, 7000 ft. Oct. Swyn. 6202.

FAmily CXVIII.-PITTOSPORACEAE, Lindl.

3252 -Pittosporum viridiflorum, Sims.

Fl. Cap. i. 443 ; Cat. Afr. Pl. Welw. i. 41 ; For. Fl. Cape, 140 Harv. Gen. S.A. Pl. 20.

Mazoe, 4200 ft. Dec. Eyles, 206, 207 ; Mt. Pene Forest, 65007000 ft. fr. Sep. Swyn. 671.

FAmily CXXI.--MYROTHAMNACEAE, Niedenzu.

3282 - Myrothamus flabellifolia, Welw.

Fl. Trop. Afr. ii. 404 ; Cat. Afr. Pl. Welw. i. 331 ; see also Fl. Cap. ii. 597, under Cliffortia? flabellifolia.

Gwelo, Dec. Rand, 5 ; Fort Gibbs, Matabeleland, Rand, 600 and 601 ; Victoria Falls, Allen, 92 ; Matopos, Galpin, 6952 ; Eyles, 1208; Engler; Nov. Marloth, 3416 : Sep. Gibbs, 309 ; Victoria, Monro, 901.

Family CXXVI.-ROSACEAE, B. Juss.

3353-Rubus Mundtii, Cham. \& Schlecht. (R. rigidus, Smith, var. Mundtii, Harv.)

Fl. Cap. ii. 287.

Melsetter, Sep. Swyn. 2077.

R. rigidus, Smith.

Fl. Trop. Afr. ii. 375 ; Fl. Cap. ii. 287.

Salisbury, July, Rand, 464; Mazoe, 4500 ft. Nov. Eyles, 472;

Chirinda, May, Swyn. 454.

3379-Leucosidea sericea, E. \& $Z$.

Fl. Cap. ii. 289 ; For. Fl. Cape, 216.

Melsetter, 6000 ft. Sep. Swyn. 605.

3388-Cliffortia linearifolia, E. \& $Z$.

Fl. Trop. Afr. ii. 379; Fl. Cap. ii. 301; For. Fl. Cape, 137.

Melsetter, $6000 \mathrm{ft}$. Swyn. 2078. 
Genus No.

3393 -Pygeum africanum, Hook. $f$.

Fl. Trop. Afr. ii. 373 ; Cat. Afr. Pl. Welw. i. 322 ; For. Fl. Port. E. Afr. 60; For. Fl. Cape, 215.

Chipete Forest, 4000 ft. April, Swyn. 1344; Chirinda, 37004000 ft. April, Swyn. 107.

3405-Parinarium capense, Harv.

Fl. Trop. Afr. ii. 368; Fl. Cap. ii. 597; Cat. Afr. Pl. Welw. i. 321 ; Harv. Gen. S.A. Pl. 94.

Salisbury, Sep. Rand, 620, 559; North of Hartley, Engler; Marandellas, Engler ; Sengwe Riv. Govt. Herb. 948; Lomagundi, Govt. Herb. 972.

P. curatellæfolium, Planch.

Fl. Trop. Afr. ii. 368.

Chikore Hills, 3500 ft. bud, Nov. Swyn. 30 ; Chirinda, $3700 \mathrm{ft}$. Swyn. 306.

P. Gilletii, De Wild.

Chirinda, Oct. Swyn. 555, 1303.

P. Mobola, Oliv.

Fl. Trop. Afr. ii. 368 ; For. Fl. Port. E. Afr. 61 ; Cat. Afr. Pl. Welw. i. 320.

Salisbury, Sep. Rand, 548, 630; Matopos, fr. Nov. Marloth, 3375 ; fl. \& fr. Oct. Gibbs, 64 ; Davy ; Engler; Govt. Herb. 894 ; North of Hartley, Engler ; Marandellas, Engler ; Gwanda, Noble, 43 ; Victoria, Monro, 454, 537, North of Sengwe Riv. Govt. Herb. 945.

Family CXXVII.-CONNARACEAE, R. $B r$.

3419-Agelæa nitida, Solander.

Chirinda, 3800 ft. fl. Oct. fr. Feb. Swyn. 228, $228 a$.

3421-Brysocarpus coccineus, Schum. \& Thonn.

Fl. Trop. Afr. i. 452.

Victoria Falls, Rogers, 5469.

var. parvifolius, Planch.

Fl. Trop. Afr. i. 452.

Victoria Falls, Sep. Gibbs, 154.

3424-Rourea sp.

Victoria Falls, Allen, 414.

3428-Cnestis natalensis, Planch. \& Sond.

Fl. Cap. i. 528.

Chirinda, 3700-4000 ft. Dec. Swyn. 166. 
FAMILy CXXVIII.--LEGUMINOSAE, Juss.

Genus No.

3443 - Albizzia amara, Boiv.

Fl. Trop. Afr. ii. 356.

Bulawayo, $4500 \mathrm{ft}$. Oct. Eyles, 1090.

A. anthelmintica, Bron.

Fl. Trop. Afr. ii. 357 ; Cat. Afr. Pl. Welw. i. 314 ; For. Fl. Port. E. Afr. 60.

Sabi Riv. 1000 ft. fr. Nov. Swyn. 1005 ; Victoria Falls, Rogers, 5319 ; Allen, 174 ; Matopos, Rogers, 5343.

A. Antunesiana, Harms.

Salisbury, Engler.

A. fastigiata, E. Mey. (Zygia fastigiata, E. Mey.)

Fl. Trop. Afr. ii. 361 ; For. Fl. Port. E. Afr. 59 ; For. Fl. Cape, 213 ; Nat. Pl. 27 ; Cat. Afr. Pl. Welw. i. 317 ; Fl. Cap. ii. 285.

Chirinda Forest, Swyn. 54 (forma).

var. chirindensis, Swyn.

Journ. Linn. Soc. Bot. xl. 65.

Chirinda Forest, 3700-4000 ft. Oct. Swyn. 52; and West of Mt. Mpengo, Swyn.

A. Haryeyi, Fourn. (A. hypoleuca, Oliv. ; A. pallida, Harv.)

Victoria Falls, Allen, 64; Rogers, 8675; Sabi Riv. 1000 ft. bud, Nov. Swyn. 1216; Bulawayo, Monro, 386, 377, 310 ; Victoria, Monro, 558.

A. mozambicensis, Sim.

For. Fl. Port. E. Afr. 59.

Plumtree, Govt. Herb. 2044.

A. versicolor, Welw.

Fl. Trop. Afr. ii. 359 ; Cat. Afr. Pl. Welw. i. 315.

Victoria Falls, Rogers, 5470.

\section{A. spp.}

Matopos, fl. \& fr. Oct. Gibbs, 198, 187.

Galpin, Sep. 7082.

Lomagundi, Govt. Herb. 978.

3446-Acacia albida, Del.

Fl. Trop. Afr. ii. 339; For. Fl. Port. E. Afr. 54; Cat. Afr. Pl. Welw. i. 310.

Victoria Falls, Allen, 399 ; Inyoka, Govt. Herb. 947.

. caffra, Willd.

Fl. Trop. Afr. ii. 345 ; Fl. Cap. ii. 282 ; For. Fl. Port. E. Afr. 56 ;

For. Fl. Cape, 210 ; Cat. Afr. Pl. Welw. i. 311.

Victoria, Monro, 681 ; South Melsetter, Swyn. 
Genus No.

3446 - $\mathbf{A}$. farnesiana, Willd.

Fl. Trop. Afr. ii. 346 ; Cat. Afr. Pl. Welw. i. 312.

Victoria Falls, Allen, 98 ; Gwanda, Noble, 4.

A. giraffae, Burch.

Fl. Cap. ii. 280 ; For. Fl. Cape, 213.

North of Bulawayo, Engler; South Rhodesia, Marloth.

A. horrida, Willd.

Fl. Cap. ii. 281 ; For. Fl. Port. E. Afr. 57 ; For. Fl. Cape, 211.

North of Bulawayo, Engler; Umtali, Engler; Umvumvumvu Riv. 2000 ft. April, Swyn. 59a; South Rhodesia, Marloth.

A. mimosoides, $L$.

Bulawayo, Monro, 20.

A. natalitia, E. Mey.

Fl. Cap. ii. 281 ; For. Fl. Port. E. Afr. 57.

Swynnerton in MSS. refers to this species as the common Acacia of his district, occurring at Chipete, Chirinda, South Chirinda and South Melsetter.

A. nigrescens, Oliv.

Fl. Trop. Afr. ii. 340 ; For. Fl. Port. E. Afr. 54.

Matopos, Davy; Victoria Falls, Davy ; Allen, 417 ; Rogers, 5297, 5299 ; South of Zambesi, Engler ; Victoria, Monro, 1083 ; Leopard's Mine, Govt. Herb. 959 ; Lomagundi, Govt. Herb. 992.

var. pallida, Oliv.

North of Bulawayo, Engler.

A. pennata, Willd.

Fl. Trop. Afr. ii. 345 ; Fl. Cap. ii. 283 ; Cat. Afr. Pl. Welw. i. 312 ; Nat. Pl. 244 ; For. Fl. Port. E. Afr. 56.

Chirinda Forest, 3700-4000 ft. Jan. Swyn. 84 ; Chipete Forest, Swyn.

A. rehmanniana, Schinz.

Bulawayo, Davy ; Rogers, 5488 ?

A. Rovumae, Oliv.

Fl. Trop. Afr. ii. 353.

Matopos, Rogers, 5346 ?

A. Seyal, Del.

Fl. Trop. Afr. ii. 351 ; For. Fl. Port. E. Afr. 57.

Bulawayo, Jan. Rand, 72.

var. multijuga, Schweinf.

Bulawayo, Dec. Rand, 37.

A. Suma, Kurz.

Victoria Falls, Allen, 68. 
Ä. Yerek, Guill. \& Perr. (A. Senegal, Willd.)

Fl. Trop. Afr. ii. 342 ; Cat. Afr. Pl. Welw. i. 311.

Bulawayo, Rogers, 5741 ; Victoria Falls, Rogers, 5544.

A. Welwitschii, Oliv.

Fl. Trop. Afr. ii. 341 ; For. Fl. Port. E. Afr. 55.

Victoria Falls, Sep. Gibbs, 128.

A. xanthophlœa, Benth.

For. Fl. Port. E. Afr. 58.

Wankie, Davy.

\section{A. spp.}

Victoria Falls, April, Flanagan, 3093 ; Rogers, 5120, 5583, 5584.

Bulawayo, Dec. Gardner, 89; Monro, 26, 32, 34, 116; Rand, 449, 450 ; Eyles, 1088 ; Rogers, 5490, 5510. ${ }^{\mathrm{r}}$

Salisbury, Rand, 4, 35, 70, 434.

Victoria, Monro, 453, 769, 388, 325, 488.

Lomagundi, Govt. Herb. 971, 981.

Charter, Govt. Herb. 3020.

South Rhodesia, Rand, 597, 598.

3449-Mimosa asperata, $L$.

Fl. Trop. Afr. ii. 335.

Victoria Falls, Allen, 65 ; April, Flanagan, 3111 ; Rogers, 5069, 7404.

3452-Dichrostachys nutans, Benth.

Fl. Trop. Afr. ii. 333; Fl. Cap. ii. 278; Nat. Pl. 243 ; For. Fl. Port. E. Afr. 53.

Bulawayo, Jan. Rand, 35 ; Eyles, Dec. 1212 ; Rogers, 5507 ; Victoria, Monro, 388 ; Gwanda, Noble, 67 ; Chirinda, $3500 \mathrm{ft.}$ Oct. Swyn. 162; 2000 ft. Dec. Swyn. 1013; Chikore Hills, 3500 ft. Oct. Swyn. 1015; South Melsetter, 2000-3500 ft. Nov. Swyn. 1016 ; Lusitu Riv. Swyn. 1019 ; Victoria Falls, Rogers, 5394, 7449 .

D. spp.

Bulawayo, Monro, 29.

Rusapi, Mundy, ef. D. nutans, Benth.

Charter, Govt. Herb. cf. D. nutans, Benth.

South Rhodesia, Rand, 314.

3457-Tetrapleura sp.

Victoria Falls, Allen, 424, cf. T. andongensis, Welw.

3458-Amblygonocarpus Schweinfurthii, Harms.

Victoria Falls, Galpin, 6949.

A. obtusangulus, Harms.

Victoria Falls, Rogers, 5970*.

I According to Rogers, his No. 5510 is Acacia robusta, Burch. 
Genus No.

3463-Piptadenia Buchanani, Baker.

Upper Buzi, 3500 ft. Oct. Swyn. 1018; South of Lusitu Riv. Swyn.; between Inyamadzi and Buzi, Swyn.

\section{P. spp.}

Victoria, Monro, 798.

3467-Elephantorrhiza Burchellii, Benth.

Fl. Cap. ii. 277.

Matopos, Marloth; Engler; Rogers, 5335 ; Umtali, Engler ; Lomagundi, Govt. Herb. 970 ; Victoria Falls, Rogers, 5783 ; Victoria, Monro, 543, 488.

E. Burkei, Benth.

Matopos, Davy

E. rubescens, Gibbs.

Journ. Linn. Soc. Bot. xxxvii. 441.

Matopos, Oct. Gibbs, 184 ; Victoria Falls, Galpin, 7062.

3468-Entada sp.

South Melsetter, Swyn. cf. E. abyssinica, Steud.

3471 -Erythrophlœum pubistamineum, Henn.

Victoria Falls, Allen, 168; Rogers, 5303.

3474-Burkea africana, Hook.

Fl. Trop. Afr. ii. 320 ; Fl. Cap. ii. 271 ; Cat. Afr. Pl. Welw. i. 304 ; Harv. Gen. S.A. Pl. 90.

Matopos, Galpin, 7059 ; Marloth, 3410 ; Davy ; Gibbs ; Victoria Falls, Galpin ; Gibbs, Oct. 143 ; Rogers, 7439, 6028 ; Bulawayo, 4500 ft. Oct. Eyles, 1093 ; Engler; Umtali, Govt. Herb. 847 ; Marandellas, Govt. Herb. 873; Chirinda and Chikore Hills, Swyn.

B. sp.

Victoria Falls, Allen, 48.

3490-Copaifera coleosperma, Benth.

Fl. Trop. Afr. ii. 314 ; For. Fl. Port. E. Afr. 52.

Victoria Falls, Galpin, 7014 ; fr. Sep. Gibbs, 144 ; Rogers, 7250, 7219 ; Allen, 155; North of Bulawayo, Engler; Hartley, Engler; Salisbury, Rand, 456, 460.

C. mopane, Kirk.

Fl. Trop. Afr. ii. 315 ; For. Fl. Port. E. Afr. 51.

Bulawayo, Dec. Rand, 12; Engler; between Malindi and Victoria Falls, Galpin, 7011 ; South of Matopos, leaf, Oct. Gibbs, 204 ; Matopos, Engler; Rogers, 5161 ; Melsetter Dist. Swyn.

3504-Brachystegia appendiculata, Benth.

Fl. Trop. Afr. ii. 305.

Sebakwe, 4000 ft. Sep. Eyles; Victoria Falls, Sep. Gibbs, 145 ; Allen, 165; Matopos, Flanagan, 3096; Gwanda, Noble, 33; Marandellas, Govt. Herb. 871. 
Genus No.

B. Bragæi, Harms .

Journ. Linn. Soc. Bot. xl. 63.

Melsetter, $5000 \mathrm{ft}$. Sep. Swyn. 657.

B. globiflora, Hook.

Victoria, Monro, 541, 970; Melsetter, March, Swyn. 164 ; Chirinda, Jan. Swyn. 6586.

B. Goetzei, Harms.

Hartley, Engler ; Rusapi, Engler.

B. Randii, E. G. Baker.

Journ. Bot. 1899, 433.

Salisbury, Sep. Rand, 610, 611; Rogers, 5796; Victoria, Monro, 456, 468, 526, 664; Matopos, Govt. Herb. 872 ; Victoria Falls, Rogers, 7456 ; Sebakwe, Rogers.

B. spicæformis, Benth.

Fl. Trop. Afr. ii. 306 ; For. Fl. Port. E. Afr. 49 ; Cat. Afr. Pl.

Welw. i. 298 \& 300 ; Journ. Linn. Soc. Bot. xl. 63.

Hartley, Engler ; Salisbury, Engler ; May, Flanagan, 3149 ;

Umtali, Engler.

B. tamarindoides, Welw.

Fl. Trop. Afr. ii. 307 ; For. Fl. Port. E. Afr. 50 ; Cat. Afr. Pl.

Welw. i. 301.

Matopos, $5000 \mathrm{ft}$. Oct. Eyles, 1250.

B. spp.

Victoria Falls, Galpin, 7057.

Victoria, Monro, 329, 330, 373.

Umtali, Govt. Herb. 849.

Lomagundi, Govt. Herb. 976, 987.

Marandellas, Govt. Herb. 916.

3506-Schotia brachypetala, Sond.

Fl. Cap. ii. 274 ; Nat. Pl. 390 ; For. Fl. Port. E. Afr. 51.

Victoria, Monro, 443.

S. latifolia, Jacq.

Fl. Cap. ii, 274 ; For. Fl. Cape, 206.

Abercorn, Govt. Herb. 933.

S. sp.

Victoria, Monro, 381.

3507-Baikiæa plurijuga, Harms.

Victoria Falls, Allen, 147 ; Rogers, 5715, 7256, 7163; North

Bulawayo, Engler; Dec. Eyles, 1122.

B. sp.

Between Malindi and Victoria Falls, 7047. 

Welw. i. 299.

Wankie, Hutchins ; Matopos, Galpin, 7079 ; Marloth ; Davy ; Oct. Gibbs, 275 ; Victoria Falls, Galpin ; Allen, 36 ; Gwanda, Noble, 24 ; Victoria, Monro, 568; Lomagundi, Govt. Herb. 988.

3511-Pahudia quangensis, Prain.

Lusitu Riv. 2500 ft. bud, Sep. Swyn. 1447 ; Chikore Hills, Swyn. 3516-Berlinia Eminii, Taub.

East of Salisbury, Engler; Umtali, Engler.

B. paniculata, Benth.

Fl. Trop. Afr. ii. 295 ; Cat. Afr. Pl. Welw. i. 298.

Victoria Falls, Rogers, 5396; Galpin, 7047 ?

3528-Bauhinia articulata, DC. (B. reticulata, DC.)

Fl. Trop. Afr. ii. 290 ; Cat. Afr. Pl. Welw. i. 296 ; For. Fl. Port. E. Afr. 48.

Salisbury, mature pod, May, Flanagan, 3148; Bulawayo, Rogers, 5494 ; Chirinda, 3600 ft. bud, March, Swyn. 165; Victoria Falls, Galpin, 7068; Hartley, Engler; Matabeleland, Marloth.

B. fassoglensis, Kotschy.

El. Trop. Afr. ii. 286.

Matabeleland, Oates; Bulawayo, Dec. Rand, 78; Jan. Gardner, 77 ; Rogers, 5503 ; Chubb, 2; Salisbury, Darling, in Herb. Bolus, 11189; Rand, 1343, 1344; Gwanda, Noble, 15; Matopos, Rogers, 5161b; Umtali, Swyn.

B. Galpini, N. E. Br.

Umtali, Engler; Lomagundi, Govt. Herb.; Victoria Falls, Rogers, 5328; between Salisbury and Portuguese border, Rogers, 12924 ; Chirinda, 3500 ft. Dec. Swyn. 192 ; Chikore Hills, Swyn.; between Melsetter and Umtali, Swyn.; Bulawayo, Eyles, 571.

B. macrantha, Oliv.

Fl. Trop. Afr. ii. 289.

Victoria Falls, Rogers, 5576, 7247 ; North of Bulawayo, Eyles, 340 .

B. petersiana, Bolle.

Fl. Trop. Afr. ii. 288.

Shashi Riv. Dec. Rand, 2 ; Victoria Falls, Rogers, 5141; between Melsetter and Umtali, Swyn.

B. spp.

Sebakwe, 4000 ft. Nov. Eyles, 172.

Victoria Falls, Allen, 137.

South Rhodesia, Rand, 315. 
Genus No

3530-Dialium guineense, Willd.

Fl. Trop. Afr. ii. 283 ; Cat. Afr. Pl. Welw. i. 294.

Victoria Falls, Rogers, 5307.

3536-Cassia abbreviata, Oliv.

Fl. Trop. Afr. ii. 271.

Matopos, Nov. Marloth, 3383 ; Engler; Mazos, 4700 ft. Sep. Eyles, 435 ; Victoria Falls, Allen, 35 ; Inyoka, Govt. Herb. 949 ; Lomagundi, Govt. Herb. 979 ; Salisbury, Govt. Herb. 936 ; Bulawayo, Chubb, 359 ; Sebakwe, Rogers, 6199.

\section{Absus, $L$.}

Fl. Trop. Afr. ii. 279 ; Cat. Afr. Pl. Welw. i. 292.

Victoria Falls, April, Flanagan, 3095, 3130 ; Rogers, 5728 ?.

C. arachoides, Burch.

Fl. Cap. ii. 272.

Bulawayo, Rand, 3 and 42; Rogers, 5824; Dec. Eyles, 48;

Nov. Eyles, 1108; Matabeleland, Marloth.

C. didymobotrya, Fres.

Fl. Trop. Afr. ii. 276 ; Cat. Afr. Pl. Welw. i. 292.

Bulawayo, July, Rand, 451; Victoria, Monro, 379 ; Salisbury, Mundy, Chirinda, 3500 ft. June, Swyn. 1419.

C. fistula, $L$.

Crescens Block, Matabeleland, Govt. Herb. 957.

C. goratensis, Fres.

Fl. Trop. Afr. ii. 273 ; Cat. Afr. Pl. Welw. i. 291.

Victoria Falls, Rogers, 5152 ; Salisbury, Govt. Herb. 759; Bulawayo, Govt. Herb. 917 ; Victoria, Monro, 362.

C. granitica, E. G. Baker.

Journ. Bot. 1905, 45.

Bulawayo, 4500 ft. Sep. Eyles, 1080 ; Matopos, Sep. Galpin, 7061 ; Davy; Oct. Gibbs, 97 ; Victoria Falls, Galpin; Davy.

C. mimosoides, $L$.

Fl. Trop. Afr. ii, 280 ; Fl. Cap. ii. 273 ; Cat. Afr. Pl. Welw. i. 293 .

Matopos, fl. \& fr. Oct. Gibbs, 251; Victoria Falls, Allen, 8; Rogers, 5044 ; Victoria, Monro, 882, 1046 ; Salisbury, Rogers, 4073 ; Chirinda, Swyn.; Bulawayo, Rogers, 2824.

C. occidentalis, $L$.

Fl. Trop. Afr. ii. 274; Fl. Cap. ii. 272 ; Cat. Afr. Pl. Welw. i. 291. Victoria Falls, April, Flanagan, 3112 ; Rogers, 5727; Victoria, Monro, 896a; Salisbury, Kolbe, 4234.

\footnotetext{
${ }^{1}$ Rogers has this number named $C$. Tora, L.
} 
C. petersiana, Bolle.

Fl. Trop. Afr. ii. 272.

Umtali, Engler; Melsetter, 3700 ft. April, Swyn. 199; $6000 \mathrm{ft}$.

Sep. Swyn. 673; Umvumvumvu Mts. 4000 ft. April, Swyn. 1155 ; Salisbury, Govt. Herb. 759 ; Odzani River Valley, Umtali, Teague, 115.

C. tettensis, Bolle.

Fl. Trop. Afr. ii. 273.

Mazoe, 4300-4800 ft. June, Eyles, 372; April, Flanagan, 3102 ;

Queque, Rogers, 6198*; Victoria Falls, Rogers, 7533a*.

C. Tora, $L$.

Fl. Trop. Afr. ii. 275; Cat. Afr. Pl. Welw. i. 292 ; Victoria Falls, Rogers, 7031*; 7441*, 13305*.

\section{C. spp.}

Bulawayo, Monro, 63; Chubb, 361 ; Govt. Herb. 939.

Victoria, Monro, 449.

North of Bulawayo, Dec. Eyles, 1123.

Chikore Hills, 4500 ft. Nov. Swyn. 1472 (cf. C. abbreviata, Oliv.).

3544-Gleditschia africana, Welw.

Fl. Trop. Afr. ii. 265 ; Cat. Afr. Pl. Welw. i. 289, 315.

Victoria Falls, Sep. Gibbs, 137 ; Rogers, 7458, 5972.

3553-Peterolobium lacerans, $R$. Br. (Cantuffa exosa, Gmelin.)

Fl. Trop. Afr. ii. 264.

Matopos, Sep. Gibbs, 81; April, Flanagan, 3097; Engler; Mazoe, 4300 ft. March, Eyles, 282 ; fr. April, Flanagan, 3098; North Melsetter, 2000-6000 ft. fr. April, Swyn. 1044; South Rhodesia, Rand.

3591-Peltophorum africanum, Sond.

Fl. Trop. Afr. ii. 260 ; Fl. Cap. ii. 270 ; Cat. Afr. Pl. Welw. i. 287; For. Fl. Port. E. Afr. 47.

Bulawayo, Rand, 10 and 318 p.p.; Nov. Eyles, 1213 ; Rogers, 5476; Marloth; Galpin, 7009; Matopos, Hutchins; Oct. Gibbs, 307 ; Engler; Victoria Falls, Allen, 66; Inyamadzi Valley, 3300 ft. Dec. Swyn. 156; Chirinda, 3500 ft. fr. Jan. Swyn. 1020 ; Chikore Hills, Swyn.; South Melsetter, Swyn.

3574-Swartzia madagascariensis, Desv.

Fl. Trop. Afr. ii. 257 ; For. Fl. Port. E. Afr. 46.

Salisbury, Rand, 1364; Victoria, Monro, 574; Mrewas, Govt. Herb. 867; Lomagundi, Govt. Herb. 994; Umtali, Swyn. ; Umvumvumvu Mts. Swyn.; Charter, Govt. Herb. 3018; Victoria Falls, Rogers, 7232.

S. sp.

Mazoe, fr. April, Flanagan, 3147. 
Genus No.

3597-Ormosia angolensis, Baker.

Fl. Trop. Afr. ii. 255 ; Cat. Afr. Pl. Welw. i. 286.

Chikore Hills, 3500 ft. fr. March, Swyn. 1473 ; Chirinda, $3000 \mathrm{ft}$. Swyn.; Victoria Falls, Rogers, 7475*, 7520*.

3601-Castanospermum australe, A. Cunn.

Salisbury, Govt. Herb. 2043.

3602-Sophora? zambesiaca, Baker.

Fl. Trop. Afr. ii. 253.

Sabi Riv. 1000 ft. Nov. Swyn. 1452.

3607-Calpurnia aurea, Baker. (C. lasiogyne, E. Mey.)

Fl. Trop. Afr. ii. 253 ; Fl. Cap. ii. 267 ; Cat. Afr. Pl. Welw. i. 286 ; Nat. Pl. 4 ; For. Fl. Cape, 205.

Chirinda, 3700-4000 ft. May, Swyn. 79 ; Chipete Forest, 3800 ft. Swyn.

3607 -Bolusanthus speciosus (Bolus), Harms. (Lonchocarpus speciosus?)

For. Fl. Port. E. Afr. 44.

Victoria Falls, Sep. Gibbs, 126 ; Allen, 169; Rogers, 5314, 7239 ; Matopos, Oct. Gibbs; Bulawayo, Chubb, 301 ; Rogers, 5500 ; Mazoe, Govt. Herb. 928 ; Umtali, Govt. Herb. 823 ; Umvumvumvu Riv. 4000 ft. Oct. Swyn. 6167.

\section{B. spp.}

Sebakwe, 4000 ft. Sep. Eyles, 176.

Victoria, Monro, 512.

Bulawayo, 4500 ft. Sep. Eyles, 1079 ; Dec. Chubb, 300.

3612 -Baphia spp.

Salisbury, Govt. Herb. 1022.

Victoria Falls, Rogers, 5586, 7270, 7233.

3657-Lotononis aristata, Schinz, var. gazensis, Baker $f$.

Journ. Linn. Soc. Bot. xl. 51.

Chimanimani Mts. 7000 ft. Sep. Swyn. 1418; Melsetter, $6000 \mathrm{ft.}$ Oct. Swyn. 6196.

L. Leobordea, Benth.

Fl. Trop. Afr. ii. 5 ; Fl. Cap. ii. 61.

Bulawayo, May, Rand, 308, 309 ; Matopos, Sep. Gibbs, 19 ; Victoria Falls, April, Flanagan, 3087.

3658-Listia heterophylla, E. Mey.

Fl. Cap. ii. 66 ; Harv. Gen. S.A. Pl. 74.

Bulawayo, May, Rand, 312 ; Rogers, 5485 ; between Bulawayo and Salisbury, April, Flanagan, 3125; Matopos, Gibbs ; Engler; Umtali, Engler; Rogers, 4028.

3659-Rothia hirsuta, Baker.

Fl. Trop. Afr. ii. 7 ; Cat. Afr. Pl. Welw. i. 195.

Bulawayo, April, Flanagan, 3088. 
Genus No. 3664-Dichilus lebeckoides, $D C$.

Fl. Cap. ii. 77.

Bulawayo, 4400 ft. Dec. Eyles, 1004.

3669-Crotalaria anthyllopsis, Welw.

Fl. Trop. Afr. ii. 15 ; Cat. Afr. Pl. Welw. i. 197 ; Journ. Linn.

Soc. Bot. xlii. 263.

Salisbury, May, Flanagan, 3101.

C. Barnabassii, Dinter.

Journ. Linn. Soc. Bot. xlii. 356.

Victoria Falls, Rogers, 5598.

C. brevidens, Benth.

Fl. Trop. Afr. ii. 37 ; Journ. Linn. Soc. Bot. xlii. 329.

Salisbury, April, Flanagan, 3118.

C. capensis, Jacq.

Fl. Trop. Afr. ii. 38; Fl. Cap. ii. 46 ; Nat. Pl. 92 ; Journ. Linn. Soc. Bot. xlii. 405.

Matopos, Govt. Herb. 900 (cultivated); Victoria Falls, Rogers, 5599.

C. cephalotes, Steud.

Fl. Trop. Afr. ii. 23 ; Cat. Afr. Pl. Welw. i. 199 ; Journ. Linn. Soc. Bot. xlii. 277.

Salisbury, fr. July, Rand, $463 a$, p.p.; Mazoe, 4600 ft. April, Eyles, 370 ; Enterprise, April, Flanagan, 3104.

C. chirindae, Baker $f$.

Journ. Linn. Soc. Bot. xlii. 377.

Chirinda, 3800 ft. Swyn. 397, 1498.

C. cylindrostachys, Welw.

Fl. Trop. Afr. ii. 15 ; Cat. Afr. Pl. Welw. i. 197 ; Journ. Linn. Soc. Bot. xlii. 257.

Victoria, Monro, 1045.

C. flavicarinata, Baker $f$.

Journ. Linn. Soc. Bot. xxxvii. 437, and xlii. 333.

Victoria Falls, Sep. Gibbs, 168; Allen, 31 ; Rogers, 5123.

C. gazensis, Baker $f$.

Journ. Linn. Soc. Bot. xl. 51, and xlii. 396.

Nyahodi Riv. 5000 ft. fl. \& fr. April, Swyn. 1493.

C. intermedia, Kotschy.

Fl. Trop. Afr. ii. 37 ; Cat. Afr. Pl. Welw. i. 203 ; Journ. Linn. Soc. Bot. xili. 327.

Mazoe, 4800 ft. March, Eyles, 274 ; Salisbury, April, Flanagan, 3119 ; South Rhodesia, Rand, 44. 
Journ. Linn. Soc. Bot. xlii. 318.

Shashi Riv. Jan. Rand, 45; Bulawayo, 4500 ft. Feb. Eyles, 1061 ;

Victoria Falls, Allen, 107, 125; Rogers, 5612; Victoria, Monro, 737 ; Manica, Johnston, in Herb. Kew.

C. lachnocarpa, Hochst. (C. elata, Welw.)

Fl. Trop. Afr. ii. 29 ; Cat. Afr. Pl. Welw. i. 201 ; Journ. Linn.

Soc. Bot. xlii. 404.

Chirinda, $3500 \mathrm{ft}$. April, Swyn. 373.

C. lanceolata, E. Mey. (C. mossambicensis, Klotsch.)

Fl. Trop. Afr. ii. 36 ; Fl. Cap. ii. 43 ; Nat. Pl. 291 ; Journ. Linn. Soc. Bot. xlii. 343 .

Chirinda, 3700-4000 ft. fl. \& fr. May, Swyn. 479.

C. natalitia, Meisn. (C. kilimandscharica, Taub.)

Fl. Trop. Afr. ii. 34 ; Fl. Cap. ii. 46 ; Cat. Afr. Pl. Welw. i. 203 ; Journ. Linn. Soc. Bot. xlii. 410.

Salisbury, Rogers, 4024 ; Chipete Forest, $3800 \mathrm{ft}$. May, Swyn. 478; Nyahodi Riv. 5000 ft. April, Swyn. 1494; Victoria, Monro, 1779; Odzani River Valley, Umtali, Teague, 22.

C. orthoclada, Welw.

Fl. Trop. Afr. ii. 29 ; Cat. Afr. Pl. Welw. i. 201; Journ. Linn. Soc. Bot. xlii. 369.

Salisbury, Govt. Herb. 756.

C. petiolaris, Franch. var. australis, Baker $f$.

Journ. Linn. Soc. Bot. xlii. 319.

Victoria, Monro, 651.

C. pisicarpa, Welw.

Fl. Trop. Afr. ii. 16 ; Cat. Afr. Pl. Welw. i. 197 ; Journ. Linn. Soc. Bot. xlii. 303.

Bulawayo, April, Flanagan, 3091.

C. podocarpa, $D C$.

Fl. Trop. Afr. ii. 17 ; Fl. Cap. ii. 593 ; Journ. Linn. Soc. Bot. xlii. 406.

Bulawayo, Jan. Rand, 43 ; April, Flanagan, 3092 ; Victoria Falls, April, Flanagan, 3128.

C. recta, Steud.

Fl. Trop. Afr. ii. 40 ; Journ. Linn. Soc. Bot. xlii. 352.

Salisbury, April, Flanagan, 3120; Chirinda, 3800 ft. Nov. Swyn. 365.

C. Rogersii, Baker $f$.

Journ. Linn. Soc. Bot. xlii. 347.

Salisbury, Rogers, 4011; Darling, in Herb. Kew 10771; Rand, 455 ; Mazoe, 4300 ft. Aug. Eyles, 181. 
C. sericifolia, Harms., var. gweloensis, Baker $f$. Journ. Linn. Soc. Bot. xlii. 398. Gwelo, fl. \& fr. Jan. Rand, 50.

C. spartioides, $D C$. Fl. Cap. ii. 40 ; Journ. Linn. Soc. Bot. xlii. 270.

Bulawayo, 4500 ft. Feb. Eyles, 1192 ; Victoria Falls, Allen, 226.

C. sphærocarpa, Guill. \& Perr.

Fl. Trop. Afr. ii. 23 ; Journ. Linn. Soc. Bot, xlii. 289.

Victoria Falls, Rogers, 5123a, 7170 ; Bulawayo, Rogers, 5921.:

var. angustifolia, Hochst. (C. mubica, Benth.)

Fl. Trop. Afr. ii. 23 ; Fl. Cap. ii. 45.

Bulawayo, April, Flanagan, 3090 ; Victoria Falls, Rogers, 5555, 7197.

C. striata $D C$.

Fl. Trop. Afr. ii. 38 ; Fl. Cap. ii. 44 ; Cat. Afr. Pl. Welw. i. 203 ; Nat. Pl. 532 ; Journ. Linn. Soc. Bot. xlii. 345. Salisbury, Engler.

\section{C. spp.}

Victoria Falls, April, Flanagan, 3110.

Gwelo, Jan. Gardner, 38, cf. C. stenoptera, Welw.

Redbank, $4000 \mathrm{ft}$. March, Eyles, 4.

Sebakwe, $4000 \mathrm{ft}$. Dec. Eyles, 74 .

Bulawayo, 4500 ft. Dec. Eyles, 84, cf. C. obscura, DC.; Monro, 99.

Victoria, Monro, 1047.

Salisbury, Govt. Herb. 757.

Charter, Govt. Herb. 3022.

3673-Argyrolobium andrewsianum, Steud.

Fl. Cap. ii. 75.

Chirinda, Swyn.; Melsetter, Swyn.

A. collinum, $E$. \& $Z$.

Fl. Cap. ii. 72.

Umtali, Rogers, 4029.

A. spp.

Bulawayo, $4500 \mathrm{ft}$. Feb. Eyles, 1175.

South Rhodesia, Rand, 60.

3702-Indigofera adenoides, Baker $f$.

Matopos, April, Flanagan, 3136.

I. arrecta, Hochst.

Fl. Trop. Afr. . 97 ; Nat. Pl. 287.

Salisbury, April, Flanagan, 3142 ; Chikore Hills, 3500 ft. March, Swyn. 260 ; Bulawayo, Rogers, 6201.

1 According to Rogers this is C. squarrosa, Schinz, var. Dinteri, Bkr. fil. 
Kew Bull. 1906, 101.

Inyanga, Cecil, 186 ; Chirinda, 3800 ft. fl. \& fr. Nov. Swyn. 262 ; fl. \& fr. April, Swyn. 263.

I. cryptantha, Benth.

Fl. Cap. ii. 195.

Matopos, fr. Sep. Gibbs, 303.

I. daleoides, Benth.

Fl. Trop. Afr. ii. 95 ; Fl. Cap. ii. 200 ; Cat. Afr. Pl. Welw. i. 214.

Bulawayo, 4500 ft. Nov. Eyles, 1218 ; Matopos, April, Flanagan, 3135 ; between Plumtree \& Bulawayo, fr. April, Flanagan, 3134.

I. diphylla, Vent.

Fl. Trop. Afr. ii. 74.

Victoria Falls, April, Flanagan, 3132, 3145 ; Rogers, 7417.

I. emarginella, Steud.

Fl. Trop. Afr. ii. 99 ; Cat. Afr. Pl. Welw. i. 216.

Chikore Hills, $3500 \mathrm{ft}$. March, Swyn 1471.

I. endecaphylla, Jacq.

Fl. Trop. Afr. ii. 96 ; Fl. Cap. ii. 199 ; Cat. Afr. Pl. Welw. i. 214.

Chirinda, 3800 ft. fr. April, Swyn. 371; fr. May, Swyn. 480 ; Victoria Falls, Rogers, 7109*.

I. filipes, Benth.

Fl. Cap. ii. 198.

Victoria Falls, April, Flanagan, 3094

I. Flanagani, Bolus, MS.

Victoria Falls, Rogers, 7206.

I. flavicans, Baker.

Fl. Trop. Afr. ii. 73.

Victoria Falls, Rogers, 7267, 5730.

I. goniodes, Hochst.

Fl. Trop. Afr. ii. 85.

Bulawayo, Jan. Rand, 38

I. hedyantha, $E . \& Z$.

Fl. Cap. ii. 188.

Mt. Pene, 7000 ft. Sep. Swyn. 2094.

I. heptaphylla, Hiern.

Cat. Afr. Pl. Welw. i. 209.

Bulawayo, Rogers, 5922, 5923 ?

I. heterotricha, $D C$.

Fl. Cap. ii. 189.

Bulawayo, 4500 ft. Nov. Eyles, 1095 ; Monro, 61 ; Rogers, 5676, 5487, 5823; Matopos, Rogers, 5407. 
Genus No.

3702-var. rhodesiana, Baker $f$.

Journ. Bot. 1903, 262.

Bulawayo, Dec. Rand, 62.

I. hilaris, $E . \& Z$.

Fl. Cap. ii. 188.

Salisbury, Aug. Rand, 445 ; Matopos, 4500 ft. Nov. Eyles, 1158 ;

Umtali, Engler; Marandellas, Rogers, 4038; Melsetter, 5000-6000 ft. Oct. Swyn. 6187, 6189; Chirinda, 3500 ft. May, Swyn. 477.

I. hirsuta, $L$.

Fl. Trop. Afr. ii. 88 ; Fl. Cap. ii. 194 ; Cat. Afr. Pl. Welw. i. 212.

Victoria Falls, April, Flanagan, 3133 ; Rogers, 7042, 7105, 5729 ;

Victoria, Monro, 1046a.

I. inyangana, N. E. Br.

Kew Bull. 1906, 102.

Inyanga, Cecil, 174.

I. Lyallii, Baker.

Chimanimani Mts. 7000 ft. fr. Sep. Swyn. 1458.

I. oxalidea, Welw.

Fl. Trop. Afr. ii. 86 ; Cat. Afr. Pl. Welw. i. 210.

Melsetter, Govt. Herb. 751.

I. pentaphylla, $L$.

Fl. Trop. Afr. ii. 82.

Salisbury, Engler.

I. procera, Schum. \& Thonn.

Fl. Trop. Afr. ii. 71 ; Cat. Afr. Pl. Welw. i. 207.

Chipetzana, 3000 ft. fr. April, Swyn. 1485.

I. Schimperi, Jaub. \& Spach.

Fl. Trop. Afr. ii. 93.

Bulawayo, Dec. Rand, 39 (forma); Victoria Falls, Rogers, 5567.

I. secundiflora, Poir.

Fl. Trop. Afr. ii. 94.

South Rhodesia, Rand, 609.

I. senegalensis, Lam.

Fl. Trop. Afr. ii. 102.

Victoria Falls, April, Flanagan, 3139 ; Rogers, 6010 ; between Bulawayo and Plumtree, April, fr. Flanagan, 3131.

I. sericea, Benth. forma australis, E. G. Baker.

Journ. Bot. 1903, 235 ; Fl. Trop. Afr. ii. 76 (type).

Bulawayo, Rand, 59.

I. strobilifera, Hochst.

Fl. Trop. Afr. ii. 75.

Victoria Falls, Rogers, 7026*. 
Genus No.

I. transyaalensis, $B k$. fil.

Bulawayo, Rogers, 13672*.

\section{I. spp.}

Victoria Falls, April, Flanagan, 3138, 3137, 3144 ; Rogers, 6020, $6014,6023$.

Salisbury, April, Flanagan, 3143; Darling in Herb. Bolus 11188 ; Govt. Herb. 755.

Bulawayo, Jan. Gardner, 62, 57, 11, 58.

Matopos, April, Flanagan, 3140 ; Rogers, 5686.

Mazoe, 4300-4700 ft. Nov. Eyles, 444.

Chikore Hills, Swyn. 267.

Melsetter, Swyn. 1459, 1460.

Chipetzana Riv. Swyn. 1486.

3703-Psoralea foliosa, Oliv. var. gazensis, Baker $f$.

Journ. Linn. Soc. Bot. xl. 52.

Melsetter, 6000 ft. Sep. Swyn. 1417.

3718-Tephrosia Apollinea, DC.

Fl. Trop. Afr. ii. 124.

Victoria Falls, Allen, 258.

T. Forbesii, Baker.

See note Fl. Trop. Afr. ii. 116.

Bulawayo, Dec. Eyles, 1206.

T. grandiflora, Pers.

Fl. Cap. ii. 209.

Chirinda, 3800 ft. fl. \& fr. April, Swyn. 369

T. lupinifolia, $D C$.

Fl. Trop. Afr. ii. 107 ; Fl. Cap. ii. 204.

Victoria Falls, April, Flanagan, 3127 : Rogers, 5716, 5605.

var. digitata, $D C$.

Fl. Trop. Afr. ii. 107.

Matopos, 4500-5000 ft. Nov. Eyles, 1154

T. lurida, Sond.

Fl. Cap. ii. 208.

Bulawayo, Jan. Rand, 41 ; Dec. Rand, 51 ; Matopos, fl. \& young fr. Oct. Gibbs, 249 ; Rogers, 5682 ; Mazoe, 4700 ft. March, Eyles, 265.

T. nyasae, Baker $f$.

Chimanimani Mts. 7000 ft. Sep. Swyn. 1464.

T. polystachya, E. Mey.

Fl. Cap. ii. 206.

Victoria Falls, Rogers, 5626, 5558. 
T. purpurea, Pers.

Fl. Trop. Afr. ii. 124.

Victoria Falls, Rogers, 7041*.

T. radicans, Welw.

Fl. Trop. Afr. ii. 121.

Matopos, April, Flanagan, 3099 ; Bulawayo, Rogers, 5486.

var. rhodesiaca, Baker $f$.

Journ. Bot. 1899, 430.

Bulawayo, fl. \& fr. Dec. Rand, 52.

\section{T. spp.}

Bulawayo, Dec. Gardner, 95, cf. T. bracteolata, Guill. \& Perr. Feb. Eyles, 1062.

Victoria, Monro, 840.

3719-Mundulea suberosa, Benth.

Fl. Trop. Afr. ii. 126 ; Cat. Afr. Pl. Welw. i. 225.

Bulawayo, Dec. Rand, 77 ; Nov. Eyles, 1183; Monro, 96 ; Rogers, 5742 ; Khami, Nov. Marloth, 3400 ; Sabi Riv. 1000 ft. Swyn. 1453 ; Victoria, Monro, 464, 550 ; Matopos, Davy.

3721-Schefflerodendron gazense, Baker $f$.

Journ. Linn. Soc. Bot. xl. 55.

Chirinda Forest, 3700-4000 ft. Oct. Swyn. 13 ; Buzi Riv. Swyn.

3746-Microcharis sp.

Matopos, April, Flanagan, 1341, ef. M. Galpinii, N. E. Br.

3747 -Sesbania ægyptiaca, Pers.

Fl. Trop. Afr. ii. 134.

Gwelo, Sr. Phil. 55.

S. cinerascens, Welw.

Fl. Trop. Afr. ii. 134 ; Cat. Afr. Pl. Welw. i. 231.

Victoria Falls, April, Flanagan, 3084, 3113.

S pubescens, $D C$.

Fl. Trop. Afr. i1. 135 ; Cat. Afr. Pl. Welw. i. 231.

Victoria Falls, Engler; South of Zambesi, Engler; Matopos, Rogers, 5159 ?, 5099.

S. punctata, $D C$.

Fl. Trop. Afr. ii. 133 ; Cat. Afr. Pl. Welw. i. 230.

Bulawayo, July, Rand, 448 ; Victoria Falls, July, Kolbe, 3132 ;

Rogers, 7407; Mazoe, April, Flanagan, 3122 ; Odzani River Valley, Umtali, Teague, 15.

S. tetraptera, Hochst.

Fl. Trop. Afr. ii. 136.

Victoria Falls, April, Flanagan, 3109. 
Genus No.

\section{S. spp.}

Victoria Falls, Allen, 7, cf. S. pubescens, DC. ; May, Eyles, 144.

Salisbury, April, Flanagan, 3121.

3756-Lessertia pauciflora, Harv.

Fl. Cap. ii. 222.

Matopos, fl. \& young fr. Sep. Gibbs, 104.

L. stipulata, E. G. Baker.

Journ. Bot. 1899, 430.

Salisbury, fl. \& young fr. Sep. Rand.

L. sp.

South Rhodesia, Rand, 614.

3766-Astragalus burkeanus, Benth.

Fl. Cap. ii. 224 ; Harv. Gen. S.A. Pl. 80.

Salisbury, Engler.

3792-Ormocarpum trichocarpum (Taub.), Torre \& Harms. (Diphaca trichocarpa, Taub.)

Bulawayo, Dec. Rand, 31.

0. spp.

Bulawayo, Eyles, 93 ; Chubb, 78 ; Gardner, 69, cf. O. bibracteatum, Baker.

3793-Eschynomene cristata, Vatke.

Victoria Falls, fl. \& fr. Sep. Gibbs ; Engler ; April, Flanagan, 3085.

A. elaphroxylon, Desv.

Victoria Falls, Rogers, 5632 ?

A. gazensis, Baker $f$.

Journ. Linn. Soc. Bot. xl. 56.

Melsetter, $6000 \mathrm{ft}$. Sep. Swyn. 1457.

A. grandistipulata, Harms.

Chimanimani Mts. 7000 ft. Swyn. 1499 ; W. Johnson, 232.

A. mimosifolia, Vatke.

North of Hartley, Engler; Victoria Falls, Kolbe, 4243 ; Victoria, Monro, 783 ; Umtali, Rogers, 4056 ; Salisbury, April, Flanagan, 3116 ; Darling in Herb. Bolus, 10766.

A. nodulosa, Baker f. (Smithia nodulosa, Baker.) (A. shirensis, Taub.)

Fl. Trop. Afr. ii. 153; Journ. Linn. Soc. Bot. xl. 56.

Melsetter, $6000 \mathrm{ft}$. Sep. Swyn. 619 .

A. nyassana, Taub.

Matopos, 4500-5000 ft. Nov. Eyles, 1117.

A. oligantha, Welw.

Fl. Trop. Afr. ii. 146 ; Cat. Afr. Pl. Welw. i. 234.

Victoria Falls, April, Flanagan, 3129. 
Genus No.

Ä. uniflora, E. A. Mey.

Fl. Cap. ii. 226 ; Fl. Trop. Afr. ii. 146.

Victoria Falls, Rogers, 7062*.

\section{A. spp.}

Bulawayo, Jan. Gardner, 17, cf. A. heuchiana, Baker.

Salisbury, Rand, 459.

Mazoe, 4300 ft. Jan. Eyles, 241, ef. A. Ruppellii, Baker ; $5300 \mathrm{ft}$. Jan. Eyles, 523.

Victoria Falls, Allen, 280.

3796-Smithia strigosa, Benth. (Damapana strigosa, O. Kuntze.)

Fl. Trop. Afr. ii. 154 ; Cat. Afr. Pl. Welw. i. 237.

Chirinda, 3800 ft. April, Swyn. 264; Chipetzana Riv. 3000 ft. April, 1483 ; Mt. Pene, 7000 ft. Oct. Swyn. 6180.

S. thymodora, Baker $f$.

Journ. Linn. Soc. Bot. xl. 56.

Melsetter, $6000 \mathrm{ft}$. Swyn. 655.

3802-Stylosanthes erecta, Beauv.

Fl. Trop. Afr. ii. 156 ; Cat. Afr. Pl. Welw. i. 238.

Bulawayo, May, Rand, 310.

S. mucronata, Willd.

Fl. Trop. Afr. ii. 157.

Bulawayo, Rogers, 13076*, 13811*.

S. sp.

Victoria, Monro, 8554.

3803-Arachis hypogea, $L$.

Fl. Trop. Afr. ii. 158 ; Fl. Cap. ii. 227 ; Cat. Afr. Pl. Welw. i. 239.

South Rhodesia, Rhod. Agric. Journ. April, 1904, 135 ; a common native crop of cultivation.

3804--Zornia diphylla, Pers.

Fl. Trop. Afr. ii. 158; Cat. Afr. Pl. Welw. i. 239.

Redbank, April, Flanagan, 3187.

Z. tetraphylla, Michx.

Fl. Trop. Afr. ii. 159 ; Fl. Cap. ii. 225.

Bulawayo, Jan. Rand, 61 ; Gwai, April, Flanagan, 3123 ; Victoria, Monro, 908 ; Victoria Falls, Rogers, 5706; Chirinda, Swyn.

3807-Desmodium dimorphum, Welw. (Meibomia dimorpha, O. Kuntze.) Fl. Trop. Afr. ii. 161 ; Cat. Afr. Pl. Welw. i. 240.

Victoria Falls, Rogers, 7152.

D. lasiocarpum, DC. (D. latifolium, DC.) (Meibomia lasiocarpa,

O. Kuntze.)

Fl. Trop. Afr. ii. 162 ; Cat. Afr. Pl. Welw. i. 241.

Chirinda, Swyn. 
Genus No.

D. paleaceum, Guill. \& Perr. (Meibomia oxybractea, O. Kuntze.) Fl. Trop. Afr. ii. 166 ; Cat. Afr. Pl. Welw. i. 242.

Victoria Falls, Engler; Rogers, 5026 ; July, Kolbe, 3152; April, Flanagan, 3115.

D. Scalpe, DC. (Meibomia repanda, O. Kuntze.) Fl. Trop. Afr. ii. 164 ; Cat. Afr. Pl. Welw. i. 242.

Salisbury, April, Flanagan, 3106 ; Chirinda, Swyn.

D. sp.

Victoria Falls, April, Flanagan, 3124, ef. D. spirale, DC.

3808-Pseudarthria Hookeri, Wight \& Arn.

Fl. Trop. Afr. ii. 168; Nat. Pl. 219 ; Cat. Afr. Pl. Welw. i. 244.

Matopos, 4500 ft. Feb. Eyles, 1167; Rogers, 4067 ; Victoria, Monro, 979 ; Salisbury, Darling; Chirinda, Swyn.; Odzani River Valley, Umtali, Teague, 10.

3810-Alysicarpus rugosus, DC. (A. Wallichii, Wight and Arn.) (Fabricia rugosa, O. Kuntze.)

Fl. Trop. Afr. ii. 171 ; Fl. Cap. ii. 230 ; Cat. Afr. Pl. Welw. i. 246. Bulawayo, 4500 ft. Eyles, 86; Gwelo, Jan. Gardner, 29 ; Umtali. Engler; Salisbury, April, Flanagan, 3117.

A. Zeyheri, Harv. (Fabricia Zeyheri, O. Kuntze.)

Fl. Trop. Afr. ii. 170 ; Fl. Cap. ii. 230 ; Cat. Afr. Pl. Welw. i. 246. Bulawayo, Rogers, 5520 ; 5483.

3821-Dalbergia dekindtiana, Harms.

Umtali, Engler.

D. lactea, Vatke.

Lusitu Riv. 3000 ft. Sep. Swyn. 1468.

D. melanoxylon, Guill. \& Perr.

Fl. Trop. Afr. ii. 233 ; For. Fl. Port. E. Afr. 41.

Sabi Riv. 1000 ft. Nov. Swyn. 2093.

D. Swynnertonii, Baker $f$.

Journ. Linn Soc. Bot. xl. 60.

Nyahodi Riv. 4000 ft. Sep. Swyn. 1316.

D. spp.

Sabi Riv. Swyn. 1201, cf. D. arbutifolia, Baker.

Victoria Falls, Rogers, 5477.

3828-Pterocarpus erinaceus, Poir. (P. echinatus, DC.) (P. angolensis, A. DC.)

Fl. Trop. Afr. ii. 239 ; For. Fl. Port. E. Afr. 44 ; Cat. Afr. Pl. Welw. i. 279.

Matopos, Davy; Galpin, 6950; Oct. Gibbs, 207; Engler ; Gwanda, Noble, 26 ; North of Bulawayo, Dr. F. E. Weiss; Bulawayo, Engler; Salisbury, Engler; Lomagundi, Govt. 
Herb. 984 ; Chirinda, 3300 ft. Oct. Swyn. 41 ; Nyahodi Riv. 5000 ft. Swyn. 1439; South Melsetter, Swyn.; South. Rhodesia, Rand; Upper Buzi, Swyn.

P. melliferus, Welw.

Fl. Trop. Afr. ii. 239; Cat. Afr. Pl. Welw. i. 277.

Victoria, Monro, 604, 750.

P. sericeus, Benth.

Fl. Cap. ii. 264.

Khami Riv. Feb. Eyles, 1195; Matopos, Hutchins; Gwanda, Noble, 19 ; Umtali, Govt. Herb. 833 ; Salisbury, Govt. Herb. 932 ; Chirinda, 3500 ft. Jan. Swyn. 28; South of Chirinda, Swyn.

\section{P. spp.}

Victoria Falls, Allen, 85, cf. P. Antunessii?

Victoria, Monro, 581.

Salisbury, Rand, 1392.

3834-Lonchocarpus capassa, Rolfe. (L. laxiflorus, Guill. and Perr.)

(L. Philenoptera, Benth.) (L. violaceus, Oliv.) (Derris violacea, Harms.)

Fl. Trop. Afr. ii. 242 ; Cat. Afr. Pl. Welw. i. 281.

Matabeleland, Oates; Victoria Falls, Allen, 47 ; Gwanda, Noble, 9 ; Matopos, Govt. Herb. 887; Lomagundi, Govt. Herb. 991; North of Bulawayo, Engler; Sabi Riv. 1000 ft. young fr. Nov. Swyn. 1451, 1397 ?

3856-Abrus precatorius, $L$.

Fl. Trop. Afr. ii. 175 ; Fl. Cap. ii. 262 ; For. Fl. Port. E. Afr. 42 ; Cat. Afr. Pl. Welw. i. 247.

Sebakwe, 4000 ft. Dec. Eyles, 41 ; Victoria Falls, Galpin, 6944 ; fr. Sep. Gibbs, 306 ; Engler; Rogers; Marandellas, Govt. Herb. 883.

3861-Dumasia villosa, $D C$.

Fl. Cap. ii. 234.

Salisbury, April, Flanagan, 3146; Mazoe, 4500 ft. April, Eyles, 353 ; Chipete Forest, Swyn.

3864 - Glycine javanica, $L$.

Fl. Trop. Afr. ii. 178; Cat. Afr. Pl. Welw. i. 249.

Victoria Falls, fr. April, Flanagan, 3114; Rogers, 5136 ; Mazoe, April, Flanagan, 3082 ; Chirinda, Swyn.

3866-Te ramnus labialis, Spreng.

Fl. Trop. Afr. ii. 180 ; Fl. Cap. ii. 235 ; Cat. Afr. Pl. Welw. i. 249. Umtali, Rogers, 4032. 
Genus No.

3870-Erythrina humeana, Spreng. (E. Humei, E. Mey.)

Fl. Trop. Afr. ii. 182 ; Fl. Cap. ii. 237 ; For. Fl. Port. E. Afr. 43 ; For. Fl. Cape, 202.

Chirinda, 3500 ft. fr. June, Swyn. 603 ; also by Swyn. at Chipete, Chipetzana, Mt. Pene, and between Melsetter and Haroni Riv.

E. tomentosa, $R . B r$. (E. latissima, E. Mey.)

Fl. Trop. Afr. ii. 184; Fl. Cap. ii. 238 ; Nat. Pl. 384, 385 ; For.

Fl. Port. E. Afr. 43; For. Fl. Cape, 201.

Salisbury, July, Rand, 462 ; Engler; Matopos, Davy; Nov. Marloth, 3374; fl. \& fr. Oct. Gibbs, 73; Engler; Gwanda, Noble, 8; Victoria, Monro, 416, 455 ; Mazoe, 4500 ft. July, Eyles, 381 ; Melsetter, fl. \& young fr. Sep. Swyn. 154 ; fl. Oct. Swyn. 668 ; Umtali, Rogers, 4017.

E. sp.

Victoria, Monro, 415.

3877-Mucuna coriacea, Baker.

Fl. Trop. Afr. ii. 187.

Mazoe, 4500-4800 ft. April, Eyles, 361 ; Chirinda, 3500 ft. May, Swyn. 450; Chipetzana Riv. 3000 ft. April, Swyn. 1487; Nyahodi Riv. 5000 ft. April, Swyn. 1488.

3879-Calopogonium lacerans, $R$. $B r$.

Matopos, Rogers, 5167.

3882-Galactia sp.

Bulawayo, Dec. Gardner, 93, 94.

3891-Canavalia ensiformis, DC. (C. incurva, P. Thouars.)

Fl. Trop. Afr. ii. 190 ; Cat. Afr. Pl. Welw. i. 254.

Victoria Falls, Allen, 130; Rogers, 5137 ; Salisbury, Govt. Herb. 760 ; Chirinda, Swyn.

3897-Rhynchosia adenodes, E. \& Z.

Fl. Cap. ii. 254.

Mazoe, April, Flanagan, 3083.

R. antennulifera, Baker.

See note Fl. Trop. Afr. ii. 223.

Salisbury, July, Rand, 453.

R. caribaea, DC. (Dolicholus caribaeus, Hiern.)

Fl. Trop. Afr. ii. 220 ; Cat. Afr. Pl. Welw. i. 267.

Mazoe, April, Flanagan, 3103.

R. clivorum, S. Moore.

Chimanimani Mts. 7000 ft. Sep. Swyn. 1461; Mt. Pene, 7000 ft. Swyn. 6184.

R. congensis, Baker. (Dolicholus angolensis, Hiern.)

Fl. Trop. Afr. ii. 217 ; Cat. Afr. Pl. Welw. i. 266.

Victoria Falls, April, Flanagan, 3107 (var.). 
Genus No.

3897 - R. cyanosperma, Benth.

Fl. Trop. Afr. ii. 218.

Chikore Hills, 3500 ft. Feb. Swyn. 226.

R. flavissima, Hochst.

Fl. Trop. Afr. ii. 219.

Chirinda, 3700-4000 ft. Oct. Swyn. 448.

R. Memnonia, DC. var. prostrata, Harv.

Fl. Trop. Afr. ii. 220 ; Fl. Cap. ii. 253 ; Nat. Pl. 349. All of type.

Victoria, Monro, 644.

R. minima, $D C$.

Fl. Trop. Afr. ii. 219 ; Fl. Cap. ii. 254.

Salisbury, Aug. Rand, 454; Victoria Falls, April, Flanagan, 3108; Rogers, 5540, 5543, 5606; Bulawayo, Chubb, 73.

R. monophylla, Schlechter.

Rusapi, Engler; Chirinda, 3800 ft. Feb. Swyn. 390, 451, 1162 ; Melsetter, 6000 ft. Swyn. 6190; Mt. Pene, 6500-7000 ft. Sep. Swyn. 1466.

R. Orthodanum, Benth.

Fl. Cap. ii. 257 ; Nat. Pl. 220.

Umtali, Engler ; Chipete, 3800 ft. Oct. Swyn. 1500.

R. puberula, Harv.

Fl. Cap. ii. 255.

Bulawayo, Jan. Rand, 46.

R. resinosa, Hochst.

Fl. Trop. Afr. ii. 218.

Salisbury, July, Rand, 457; Matopos, Sept.-Oct. Gibbs, 68 ;

April, Flanagan, 3100 ; Rogers, 5181; Victoria, Monro, 1068.

R. Totta, $D C$.

Fl. Cap. ii. 255.

Victoria Falls, Rogers, 7213 ; Bulawayo, Rogers, 13686*.

R. viscidula, Steud.

Fl. Cap. ii. 251.

Victoria, Monro, 549.

R. spp.

Gwelo, Jan. Gardner, 5.

Bulawayo, Jan. Gardner, 82 ; Oct. Eyles, 1086.

Victoria Falls, April, Flanagan, 3089.

Salisbury, April, Flanagan, 3105, same as Bolus, 10773 ; Salisbury,

Darling, in Herb. Bolus, 10773, 10774, 11186, 11187 ; Rand, 452 ; Rogers, 4051, 4014.

Mazoe, 4300 ft. Aug. Eyles, 182 ; 4800 ft. June, Eyles, 375.

South Rhodesia, Rand, 608. 
Genus No.

3898-Eriosema Burkei, Benth.

Fl. Cap. ii. 260.

Salisbury, Rogers, 4014 ; Selukwe, Rogers, 4092.

E. cajanoides, Hook. $f$. (E. psoraleoides, G. Don.)

Fl. Trop. Afr. ii. 227 ; Fl. Cap. ii. 261 ; Cat. Afr. Pl. Welw. i. 272 .

Bulawayo, Jan. Rand, 49; fr. July, 447; Dec. Eyles, 28; Victoria Falls, Allen, 134; Umtali, Engler; Odzani River Valley, Teague, 7 ; Victoria, Monro, 926; Crocodile Riv. Holub; Chirinda, Swyn.; Chikore Hills, Swyn.; Salisbury, Rogers, 4066.

E. ellipticum, Welw.

Fl. Trop. Afr. ii. 227 ; Cat. Afr. Pl. Welw. i. 274.

Melsetter, $6000 \mathrm{ft}$. Sep. Swyn. 624.

E. Engleri, Harms.

North of Hartley, Engler, 3012 ; Salisbury, Engler, Sep. 3023 ;

Macheke, Rogers, 4044, 4061; Bulawayo, Rogers, 10773*.

E. montanum, Baker $f$.

Chirinda, $3800 \mathrm{ft}$. Sep. Swyn. 361, not typical form.

E. oblongum, Benth.

Fl. Cap. ii. 259.

Salisbury, fl. \& fr. Sep. Rand, 590 ; Rogers, 4004; North of Bulawayo, 3400 ft. Dec. Eyles, 1131; Gwelo, Sr. Phil. 22.

E. paryiflorum, E. Mey.

Fl. Cap. ii. 260 ; Fl. Trop. Afr. ii. 225.

Bulawayo, Rogers, 13674*.

E. polystachyum, Baker.

Fl. Trop. Afr. ii. 225.

Bulawayo, Jan. Rand, 48.

E. shirense, Baker $f$.

Lusitu Hills, 5000 ft. April, Swyn. 1497.

E. spp.

Bulawayo, 4500 ft. Sep. Eyles, 104, cf. E. parviflora, E. Mey.

Salisbury, Aug. Rand, 458, cf. E. Engleri, Harms, and E. insigne, O. Hoffm.

Victoria, Monro, 533, 553.

Mazoe, 4300-4800 ft. Nov. Eyles, 530.

Gwelo, Sr. Phil. 38.

3899-Flemingia rhodocarpa, Baker.

Fl. Trop. Afr. ii. 231.

Mazoe, 4600-4800 ft. July, Eyles, 382 ; Chirinda, 3700-4000 ft. July, Swyn. 1048. 
Genus No.

3901-Phaseolus lunatus, $L$.

Fl. Trop. Afr. ii. 192 ; Cat. Afr. Pl. Welw. i. 255.

Umtali, Rogers, 4055 ; South Rhodesia, Rhod. Agric. Journ. April, 1906, 362. Native crop of cultivation.

P. sp.

Bulawayo, Dec. Gardner, 88.

3905-Vigna Buchneri, Harms.

North of Hartley, Engler.

Y. Burchelli, Harv.

Fl. Trop. Afr. ii. 196 ; Fl. Cap. ii. 239.

Bulawayo, Rogers, 5497.

Y. cærulea, Baker.

Fl. Trop. Afr. ii. 203.

Gwai Riv. 3200 ft. Allen, 247.

Y. luteola, Benth. (V.glabra, Savi.)

Fl. Trop. Afr. ii. 205 ; Fl. Cap. ii. 241 ; Cat. Afr. Pl. Welw. i. 260.

Victoria Falls, April, Flanagan, 3086 ; Salisbury, Rogers, 5522? ; Chimanimani Mts. 7000 ft. Sep. Swyn. 1467 ; Mt. Pene, 7000 ft. Oct. Swyn. 6192.

var. villosa, Baker.

Fl. Trop. Afr. ii. 206 ; Cat. Afr. Pl. Welw. i. 260.

Victoria Falls, Sep. Gibbs, 142 ; Rogers, 7453, 5968.

Y. nilotica, Hook. fil.

Fl. Trop. Afr. ii. 204.

Victoria Falls, Rogers, 7110*.

Y. nuda, N. E. Br.

Kew Bull. 1901, 121.

Mashonaland, Bryce; Salisbury, Darling in Herb. Bolus, 10772 ; Bulawayo, Rogers.

Y. triloba, Walp.

Fl. Trop. Afr. ii. 204 ; Fl. Cap. ii. 241 ; Cat. Afr. Pl. Welw. i. 259.

Matopos, Sep. Gibbs, 206, forma; Victoria Fails, April, Flanagan, 3126 ; Rogers, 7111.

У. vexillata, Benth. (V. hirta, Hook.) (V. capensis, Walp.)

Fl. Trop. Afr. ii. 199 ; Fl. Cap. ii. 240; Cat. Afr. Pl. Welw. i. 257.

Salisbury, Sep. Rand, 589 ; Umtali, Engler; Victoria, Monro, 1013 ; Chirinda, 3800 ft. April, Swyn. 363 ; Umvumvumvu Riv. 4000 ft. Oct. Swyn. 6191.

\section{Y. spp.}

Victoria Falls, Allen, 160 ; May, Eyles, 139.

Gwai Forest, Jan. Allen, 245.

Matopos, 4500-5000 ft. Nov. Eyles, 1145. 
Genus No.

3907-Sphenostylis marginata, E. Mey. (Vigna marginata, Benth.)

Fl. Trop. Afr. ii. 202 ; Fl. Cap. ii. 240.

Salisbury, Rand, 461; Engler; Rogers, 4059 ?; Victoria Falls, Allen, 176; Victoria, Monro, 766 ; Chirinda, 3800 ft. April, Swyn. 364 ; South Melsetter, 3500 ft. Oct. Swyn. 6624.

3910-Dolichos euryphyllus, Harms, forma lobata.

Sebakwe, 4000 ft. Dec. Eyles, 165.

D. falciformis, E. Mey.

Fl. Cap. ii. 246.

Victoria Falls, Galpin, 7336.

D. gibbosus, Thunb.

Fl. Cap. ii. 244.

Bulawayo, Eyles.

D. Hendrickxii, Wildem.

Without locality, Rogers*.

D. Lablab, $L$.

Fl. Trop. Afr. ii. 210 ; Fl. Cap. ii. 243.

Matopos, Rogers, 5163 ; Mazoe, 4500 ft. Feb. Eyles, 526 ?

D. lupiniflorus, N. E. Br.

Kew Bull. 1906, 102.

Chirinda, 3500 ft., Nov. Swyn. 1045.

D. stipulosus, Welw. var. Randii, Baker $f$.

Journ. Bot. 1899, 432 ; Fl. Trop. Afr. ii. 212 (type).

Salisbury, Dec. Rand, 80.

D. tricostatus, Baker $f$.

Journ. Bot. 1899, 431.

Bulawayo, Jan. Rand, 82.

D. spp.

Bulawayo, Monro, 62.

Mazoe, 4800 ft. Dec. Eyles, 494.

South Rhodesia, Rand, 311.

3911-Adenodolichos?

Victoria, Monro, 673, 999.

Series GERANIALES.

FAmily CXXIX.-GERANIACEAE, J. St. Hil.

3924-Geranium caffrum, $E$. \& $Z$.

Fl. Cap. i. 258.

Melsetter, 6000 ft. fl. \& fr. Sep. Swyn. 790.

G. Thodei, R. Kunth.

Matopos, Galpin, 6591. 
Genus No

3925-Monsonia biflora, $D C$.

Fl. Trop. Afr. i. 290 ; Fl. Cap. i. 255 ; Nat. Pl. 96 ; Cat. Afr. Pl. Welw. i. 108.

Bulawayo, Dec. Rand, 19 ; Jan. Eyles, 70.

M. burkeana, Planch.

Fl. Cap. i. 255.

Salisbury, Aug. Rand, 441; Matopos, Oct. Gibbs, 222; Macheke, Rogers, 4049 ; Victoria, Monro, 852 ?

M. ovata, $C a v$.

Fl. Cap. i. 255 ; Nat. Pl. 97 (var.).

Bulawayo, 4500 ft. Jan. Eyles; Rogers, 5929 ; Matabeleland, Marloth.

\section{M. spp.}

Bulawayo, Feb. Eyles, 1174, 1193 ; Monro, 14.

South Rhodesia, Rand, 95.

3928-Pelargonium aconitophyllum, E. \& Z. (forma).

Fl. Cap. i. 276.

Mt. Pene, 7000 ft. Oct. Swyn. 6099.

P. sp.

Bulawayo, Jan. Gardner, 64.

\section{FAmily CXXX.-OXALIDACEAE, Lindl.}

3936-0xalis corniculata, $L$. var. stricta, Oliv.

Fl. Trop. Afr. i. 297 ; Cat. Afr. Pl. Welw. i. 109 ; Fl. Cap. i. 351 (type).

Bulawayo, May, Rand, 296.

0. semiloba, Sond.

Fl. Trop. Afr. i. 296 ; Fl. Cap. i. 350 ; Cat. Afr. Pl. Welw. i. 109. Salisbury, Rogers, 5773 ; Melsetter, Swyn.

0. spp.

Bulawayo, 4500 ft. Dec. Eyles, 73, cf. O. caprina, L.

Mazoe, 5000 ft. Dec. Eyles, 210 ; April, Eyles, 344.

Selukwe, Jan. Gardner, 50.

\section{Family CXXXII.-inACEAE, Dumort.}

3945-Linum Thunbergii, E. \& $Z$.

Fl. Cap. i. 310.

Matopos, Nov. Eyles, 1098 ; Matabeleland, Marloth. 
FAMILy CXXXIV.-ERYTHROXYLACEAE, Lindl.

Genus No. 3956-Erythroxylon monogynum, Roxb. (E. pictum, E. Mey.)

Sim places these all under E. monogynum, Roxb., see For. Fl. Cap. 149, and For. Fl. Port. E. Afr. 21.

Fl. Trop. Afr. i. 274 ; Fl. Cap. i. 233, 234, and ii. 591.

Victoria Falls, Rogers, 5375, 5539, 5560.

var. caffrum, O. E. Schultz.

Chipete Forest, 3800 ft. Dec. Swyn. 133; Oct. Swyn. 692 ; Chimanimani Mts. 7000 ft. bud Sep. Swyn. 1364, 1465.

\section{Family CXXXV.-ZYGOPHYLLACEAE, Lindl.}

\section{5-Zygophyllum simplex, $L$.}

Fl. Trop. Afr. i. 285 ; Fl. Cap. i. 357 ; Cat. Afr. Pl. Welw. i. 106.

Crocodile Riv. Oates.

\section{Z. sp.}

Victoria, Monro, 826.

\section{8-Tribulus terrestris, $L$.}

Fl. Trop. Afr. i. 283 ; Fl. Cap. i. 352 ; Cat. Afr. Pl. Welw. i. 105. Bulawayo, 4500 ft. Dec. (Collector ?) 7 ; Khami Riv. March, Eyles, 11; Bulawayo, Rogers, 5736; Victoria Falls, Rogers, 5395.

\section{Family CXXXVII.-RUTACEAE, Juss.}

3990-Xanthoxylum capense, Harv.

Fl. Cap. i. 446.

Victoria, Monro, 791.

4014-Thamnosma africanum, Engl.

Matopos, Engler; Rogers, 5176.

var. rhodesicum, $B a k$. $f$. Journ. Bot. 1899, 426.

Bulawayo, fl. \& young fr. Jan. Rand, 83 ; fr. May, Rand? 297 ; Matopos, Sep.-Oct. Gibbs, 310 ; Rogers, 5255.

4076-Yepris paniculata, Lam.

Victoria, Monro, 739 ?

4077-Toddalia aculeata, Pers.

Chipete Forest, fl. \& fr. Nov. Swyn. 213 ; Chirinda Forest, Swyn.

T. lanceolata, Lam. (Vepris lanceolata, A. Juss.)

Fl. Trop. Afr. i. 307 ; Fl. Cap. i. 447 ; For. Fl. Port. E. Afr. 24 ;

For. Fl. Cape, 156.

Matopos, Rogers, 5409. 
Genus No.

4085-Teclea natalensis, Engl. (Toddatia natalensis, Sond.)

Fl. Cap. i. 447.

Chimanimani Mts. 7000 ft. bud Sep. Swyn. 1322.

T. Swynnertonii, Baker $f$.

Journ. Linn. Soc. Bot. xl. 35.

Chirinda Forest, Swyn. 12.

4100-Citrus aurantium, $L$.

Cat. Afr. Pl. Welw. i. 118.

Chirinda Forest, 3700-4000 ft. Swyn. 672 ; Lusitu Riv. Swyn.

C. medica, $L$. var. limonium, Risso.

South Rhodesia, Rhod. Agric. Journ. Sep. 1904, 8; Mazoe, Eyles.

Family CXXXVIII.-SIMARUBACEAE, Lindl.

4128-Kirkia acuminata, Oliv.

Fl. Trop. Afr. i. 311 ; For. Fl. Port. E. Afr. 24.

Victoria Falls, Allen, 51, 96; Umtali, Engler; Gwanda, Noble, 12 ; Victoria, Monro, 637; Mazoe, Govt. Herb. 922; Abercorn, Govt. Herb. 935.

\section{FAmily CXXXIX.-BURSERACEAE, Kunth.}

4151-Commiphora acutidens, Engl.

Bulawayo, Engler.

C. edulis?

Victoria Falls, Rogers, 5788.

C. Fischeri, Engl.

Sabi Riv. 1000 ft. Nov. Swyn. 1208, 1450.

C. spp.

Matopos, Oct. Gibbs, 197 ; Rogers, 5341.

Gwanda, Noble, 51.

Victoria, Monro, 355 ; 637 cf. C. natalense, Sparm.

Bulawayo, Oct.-Dec. Chubb, 319.

Shashi, Baines.

\section{Family CXL.-MELIACEAE, Vent.}

4159-Khaya nyasica, Stapf.

Journ. Linn. Soc. Bot. xl. 42.

Chirinda Forest, Swyn. 15; between Chirinda and Chikore, Swyn.

4171-Turræa Eylesii, Baker $f$.

Journ. Bot. 1905, 45.

Matopos, 5000 ft. Jan. Eyles, 29 ; Davy. 
Genus No.

T. nilotica, Kotschy. \& Peyr. (Vide T. Randii, Bak. f. below.)

Fl. Trop. Afr. i. 331 ; For. Fl. Port. E. Afr. 25.

Victoria Falls, Dr. Meller; Allen, 400; Matopos' Engler;

Salisbury, Engler.

T. obtusifolia, Hochst.

Fl. Trop. Afr. j. 331; Fl. Cap. i. 245; For. Fl. Cape, 159.

Matopos, Cecil, 105 ; Victoria, Monro, 731, 1070, 1100a.

var. matopensis, Baker $f$.

Journ. Bot. 1905, 45.

Matopos, 5000 ft. Jan. Eyles, 154.

T. Randii, Baker f. (Engler says same as T. nilotica, K. \& P.)

Journ. Bot. 1899, 427.

Salisbury, July, Rand, 562; Matopos, Sep. Galpin, 7086 ; fl. \& fr.

Oct. Gibbs, 2; Victoria, Monro, 360, 507 ; Bulawayo, Oct.-

Dec. Chubb, 320 p.p. ; Victoria Falls, Rogers, 7228 ; Sebakwe, Rogers.

\section{T. spp.}

Victoria Falls, Allen, 16 ; Rogers, 5135.

Victoria, Monro, 731a.

Mazoe, 4800 ft. July, Eyles, 385.

4175-Melia azedarach, $L$.

Fl. Trop. Afr. i. 332 ; Fl. Cap. i. 245.

Salisbury, Govt. Herb. 920, cultivated.

4193-Ekebergia arborea, Baker $f$.

Journ. Bot. 1899, 427.

Salisbury, Sep. Rand, 612; Victoria, Monro, 479; Chirinda, 3700-4000 ft. fl. \& fr. Oct. Swyn. 25; Chikore, Swyn.

E. Meyeri, Presl.

Fl. Cap. i. 247 ; Nat. Pl. 6.

Chirinda Forest, 3700-4000 ft. Oct. Swyn. 18; Chirinda, 3000 ft. Swyn.

E. sp.

Victoria, Monro, 587.

4195-Trichilia chirindensis, Swyn. \& Baker $f$.

Journ. Linn. Soc. Bot. xl. 39.

Chirinda Forest, 3700-4000 ft. Swyn.

T. emetica, Vahl.

Fl. Trop. Afr. i. 335 ; Nat. Pl. 80 ; For. Fl. Port. E. Afr. 26 ;

For. Fl. Cape, 160 ; Cat. Afr. Pl. Welw. i. 134.

Victoria Falls, Sep. Galpin, 7019 ; Sep. Gibbs, 110 ; Allen, 61 ;

Rogers, 5119 ; Inyoka, Govt. Herb. 941. 
Genus No.

4198-Loyoa Swynnertonii, Baker $f$.

Journ. Linn. Soc. Bot. xl. 41.

Chirinda Forest, 3700-4000 ft. Jan. Swyn. 16.

Family CXLI.-MALPIGHiACEAE, Vent.

4219-Sphedamnocarpus Galphimiæfolius, Szyszyl. (Acridocarpus Galphimiafolius, Juss.)

Fl. Cap. i. 232.

Matopos, Oct. Gibbs, 281.

S. pruriens, Planch. (Acridocarpus pruriens, Juss.)

Fl. Cap. i. 232 ; Nat. Pl. 400.

Matabeleland, Oates; Bulawayo, May, Rand, 431; Eyles, 96 ;

Monro, 46; Matopos, Galpin, 6951; Engler; March, Flanagan, 2975 ; Rogers, 5051 ; between Plumtree and Bulawayo, April, Flanagan, 3181; Victoria, Monro, 780, 781 ; Gwelo, Sr. Phil. 20 ; Selukwe, Rogers, 4097 ; Chirinda, 3800 ft. fl. \& fr. May, Swyn. 211 (forma); Chipete, $3800 \mathrm{ft}$. April, Swyn. $211 a$ (forma).

S. pulcherimus, Gilg.

Matopos, $5000 \mathrm{ft}$. March, Eyles, 108.

S. sp.

Victoria, Monro, 875.

4237-Tricomaria macrocarpa?.

South Rhodesia, Rand, 639.

Family CXLV.-POLYGaLACEAE, Lindl.

4273-Polygala abyssinica, Fresn. (P. hottentotta, Presl.)

Fl. Trop. Afr. i. 130 ; Fl. Cap. i. 86.

Bulawayo, Dec. Rand, 14 ; Salisbury, Sep. Rand, 599 ; Matopos, Oct. Gibbs, 194; Victoria, Monro, 835, 861; Mt. Pene, 7000 ft. Dec. Swyn. 6154; Melsetter, 6000 ft. Oct. Swyn. 6157.

P. africana, Chod. (P. micrantha, Guill. \& Perr.)

Fl. Trop. Afr. i. 131 ; Cat. Afr. Pl. Welw. i. 42.

Matopos, March, Eyles, 1030.

P. amatymbica, $E . \& Z$.

Fl. Cap. i. 93.

Bulawayo, Rogers, 13547*, 13731*. 
Matopos, March, Flanagan, 2968; Bulawayo, Monro, 90; Gwelo, Sr. Phil. 19; Chirinda, 3800 ft. May, Swyn. 282 ; Victoria Falls, Rogers; Odzani River Valley, Umtali, Teague, 182.

P. capillaris, E. Mey.

Fl. Trop. Afr. i. 131; Fl. Cap. i. 93; Nat. Pl. 83.

Matopos, March, Flanagan, 2972.

P. erioptera, DC. (P. retusa, Hochst.) (P. triflora, L.)

Fl. Trop. Afr. i. 128 ; Cat. Afr. Pl. Welw. i. 46.

Bulawayo, May, Rand, 290; Chubb, 12a.

P. gazensis, Baker $f$. Journ. Linn. Soc. Bot. xl. 24.

Chimanimani Mts. 7000 ft. Sep. Swyn. 632.

P. latipetala, N.E. Br.

Kew Bull. 1906, 98.

Between Umtali and Salisbury, Cecil, 45.

P. livingstoniana, Chod.

Cat. Afr. Pl. Welw. i. 44.

Salisbury, Rand, $463 b$.

P. persicariæfolia, $D C$.

Fl. Trop. Afr. i. 129 ; Cat. Afr. Pl. Welw. i. 43.

Bulawayo, $4500 \mathrm{ft}$. Dec. Eyles, 45 ; Mazoe, $4300 \mathrm{ft}$. April, Eyles, 302 ; Victoria Falls, April, Flanagan, 3169 ; Rogers, 5667.

P. petitiana, Rich.

Fl. Trop. Afr. i. 133.

Salisbury, May, Flanagan, 3256.

P. rarifolia, DC. (P. tenuifolia, Link.)

Fl. Trop. Afr. i. 132 ; Cat. Afr. Pi. Welw, i. 47

Mazoe, 4300 ft. Dec. Eyles, 484.

P. rigens, $A . D C$

Matopos, Oct. Gibbs, 82; Feb. Eyles, 1113; Victoria, Monro, 953.

P. virgata, Thunb.

Fl. Cap. i. 85.

Crocodile Riv. Oates; Salisbury, July, Rand, 437 ; Mazoe, 4200 ft. Dec. Eyles, 198; Chirinda, Dec. Swyn. 284; Melsetter, $6000 \mathrm{ft}$. Oct. Swyn, 6158; Chimanimani Mts. $7000 \mathrm{ft}$. Sep. Swyn. 2042.

var. decora, Harv.

Fl. Cap. i. 85.

Salisbury, April, Flanagan, 3262 ; Odzani River Valley, Umtali, Teague, 95. 
Genus No.

\section{P. spp.}

Victoria Falls, Allen, 101 ; Rogers, 5698.

Victoria, Monro, 534 ; 1030 cf. P. filicaulis, Baill.

South Rhodesia, Rand, 15.

4275-Securidaca longipedunculata, Fres.

Fl. Trop. Afr. i. 134 ; Cat. Afr. Pl. Welw. i. 47 ; For. Fl. Port. E. Afr. 14.

Victoria Falls, Allen, 423; Salisbury, Rand, 1365 ; Lomagundi, Govt. Herb. 982 ; Matopos, Flanagan, 3009 ; Victoria, Monro, 328.

var. parvifolia, Oliv. (Perhaps same as S. spinosa, Sim.)

Fl. Trop. Afr. i. 134 ; For. Fl. Port. E. Afr. 14.

Matopos, fl. \& fr. Oct. Gibbs, 244 ; Umtali, Engler; Chikore Hills, 3500 ft. Swyn, 2038 ; Umvumvumvu Riv. Swyn. 6155; South Umtali, 2000-3000 ft. Swyn. 6613; South Melsetter, Swyn.

S. spp.

Victoria, Monro, 520, 567, 570.

Mazoe, Govt. Herb. 2085.

Family CXLVI.-DiChaPETALACEAE, Engl.

4283-Dichapetalum cymosum (Hook.), Torre \& Harms. (Chailletia cymosa, Hook.)

Fl. Cap. i. 450 ; Harv. Gen. S.A. Pl. 49.

Crocodile Riv.?, Oates ; Bulawayo, Govt. Herb. 938.

D. rhodesicum, Sprague \& Hutch.

Kew Bull. 1908, 433.

Gwai Forest, Allen, 234.

\section{FAmily CXLVII.-EUPHORBIACEAE, J. St. Hil.}

\section{5-Pseudolachnostylis Dekindtii, Pax.}

Fl. Trop. Afr. VI. i. 673.

Victoria Falls, Rogers, 5304; Allen, 164, 166 ; Wankie, Rogers, 5991 ; Bulawayo, Chubb, 80.

P. maprouneæfolia, $\operatorname{Pax}$.

Fl. Trop. Afr. VI. i. 672.

Victoria, Monro, 724; Matopos, Rogers, 5334; Gibbs, 276 б; Umvumvumvu Riv. 4000 ft. fr. April, Swyn. 2128.

\section{P. spp.}

Matopos, Govt. Herb. 896.

Matabeleland, Monro, 207, 436. 
A Record of Plants Collected in Southern Rhodesia.

Genus No.

4297-Securinega verrucosa (Th.), Benth.

Victoria Falls, Rogers, 5562.

4298-Flüggea microcarpa, Blume.

Fl. Trop. Afr. VI. i. 736 ; Cat. Afr. Pl. Welw. i. 961.

Sabi Riv. 1000 ft. Swyn. 1911; Umswirizwi Riv. 1000 ft. Nov. Swyn. 1727 ; Bulawayo, Rogers, 5491.

4299 - Phyllanthus floribundus, Mïll. Arg.

Fl. Trop. Afr. VI. i. 701 ; Cat. Afr. Pl. Welw. i. 957.

South of Zambesi, Engler; Victoria, Monro, 916; Wankie Rogers, 5991*.

P. graminicola, Hutch.

Fl. Trop. Afr. VI. i. 708 ; Journ. Linn. Soc. Bot. xl. 191.

Chirinda, 3800 ft. Feb. Swyn. 261.

P. hutchinsonianus, S. Moore.

Journ. Linn. Soc. Bot. xl. 192.

Chimanimani Mts. 7000 ft. fl. \& fr. Sep. Swyn. 1524.

P. maderaspatensis, $L$. (P. longifolius, Sond.)

Fl. Trop. Afr. VI. i. 722 : Cat. Afr. Pl. Welw. i. 959.

Mazoe, 4200 ft. Jan. Eyles, 245; Chirinda, Feb. Swyn. 333 ;

Bulawayo, 4500 ft. Dec. Eyles, 6; Rogers, 5765; Victoria

Falls, Rogers, 5563.

P. myrtaceus, Sond.

Fl. Trop. Afr. VI. i. 726 ; For. Fl. Cape, 325.

Mt. Pene, 6500-7000 ft. Sep. Swyn. 1522, 1523; Chimanimani Mts. 7000 ft. fr. Oct. Swyn. 6155a.

P. Niruri, $L$.

Fl. Trop. Afr. VI. i. 731 ; Cat. Afr. Pl. Welw. i. 960.

Matopos, Oct. Gibbs, 314.

P. nummulariæfolius, Poir.

Fl. Trop. Afr. VI. i. 710.

Victoria, Monro, 712, 757 ; Mazoe, 4800-5000 ft. March, Eyles, 292.

P. pentandrus, Schum. \& Thonn.

Fl. Trop. Afr. VI. i. 710 ; Cat. Afr. Pl. Welw. i. 957.

Victoria Falls, Rogers, 5564, 5703.

P. reticulatus, Poir.

Fl. Trop. Afr. VI. i. 700 ; Cat. Afr. Pl. Welw. i. 958.

Victoria Falls, fl. \& fr. Sep. Gibbs, 129 ; Rogers, 5473 ; Sep.

Galpin, 7016; Matopos, Rattray in Herb. Galpin, 6969.

\section{P. spp.}

Igusi, April, Flanagan, 3190 ; Matopos, Davy; Victoria, Monro, 347, 457, 804 ; Victoria Falls, Rogers, 5148; South Rhodesia, Rand, 210, 211, 212. 
Genus No.

4325-Hymenocardia acida, Tul.

Fl. Trop. Afr. VI. i. 651 ; Cat. Afr. Pl. Welw. i. 966.

Chikore Hills, fl. Nov. fr. March, Swyn. 1214, 1519.

4327-Antidesma membranaceum, Müll. Arg.

Fl. Trop. Afr. VI. i. 645 ; Cat. Afr. Pl. Welw. i. 965.

Chirinda, 5300 ft. ; Chipetzana, 3000 ft. ; Mt. Pene, 6500 ft. ; fl. Sep.-Dec. fr. April, Swyn. 169, 169a, 631, 1111, 2011.

A. venosum, Tul.

Fl. Trop. Afr. VI. i. 646 ; Cat. Afr. Pl. Welw. i. 965.

South of Zambesi, Engler; Victoria, Monro, 762, 1059 ; Victoria Falls, Rogers, 5373.

4329-Uapaca kirkiana, Miill. Arg.

Fl. Trop. Afr. VI. i. 636.

Salisbury, Engler; Marandellas, Engler; Umtali, Engler; Melsetter, 4000 ft. Johnson, 128 ; Chirinda, 3500 ft. Jan. Swyn. 49 ; also by Swynnerton South of Umtali, Chimanimani Mts. and throughout Melsetter Dist.

U. sansibarica, $P a x$.

Fl. Trop. Afr. VI. i. 636.

Melsetter and Chirinda, Jan.-April, Swyn. 155, 6513.

4345-Bridelia atroviridis, Miill. Arg.

Fl. Trop. Afr. VI. i. 617 ; Cat. Afr. Pl. Welw. i. 953.

Chipete Forest, $3800 \mathrm{ft}$. fr. April, Swyn. 535.

B. cathartica, Bertol. $f$.

Fl. Trop. Afr. VI. i. 617 ; For. Fl. Port. E. Afr. 106.

Victoria Falls, Rogers, 5071, 7181; Galpin, 7015.

B. micrantha, Baill.

Fl. Trop. Afr. VI. i. 620.

Chirinda, 3500 ft. Nov. Swyn. 536; also throughout South Melsetter, Swyn.

B. mollis, Hutch. (B. stipularis, Müll. Arg.)

Fl. Trop. Afr. VI. i. 613 ; For. Fl. Port. E. Afr. 106 ; Kew Bull. $1912,101$.

Victoria, Monro, 684, 790, 643a; Bulawayo, Rogers, 5499.

B. Niedenzui, Gehrm.

Fl. Trop. Afr. VI. i. 616.

Mazoe, 4300-4500 ft. March, Eyles, 273; Umvumvumvu Riv. 2000 ft. fl. \& fr. April, Swyn. 1736.

B. scandens, Willd. (B. stipularis, Blume.)

Cat. Afr. Pl. Welw. i. 955 ; For. Fl. Port. E. Afr. 106.

Bulawayo, Rogers, 5499.

B. sp.

Victoria Falls, April, Flanagan, 3154. 
Genus No.

4348-Croton barotsensis, Gibbs.

Fl. Trop. Afr. VI. i. 767 ; Journ. Linn. Soc. Bot. xxxvii. 469.

Victoria Falls, Sep. Gibbs, 109 ; Allen, 175, 416 ; Rogers, 5474 ;

Galpin, 7049.

C. gratissimus, Burch.

Fl. Trop. Afr. VI. i. 1051 ; For. Fl. Cape, 311.

Victoria Falls, Sep.-Oct. Galpin, 6989 ; Matopos, Oct. Gibbs, 209 ; Rogers, 5410; South of Zambesi, Engler; Victoria, Monro, 322, 306, 810.

C. Gubouga, S. Moore.

Fl. Trop. Afr. VI. i. 766.

Sabi Riv. 1000 ft. Nov. Swyn. 1123.

C. rivularis, Müll. Arg.

For. Fl. Cape, 311.

South of Zambesi, Engler.

C. sylyaticus, Hochst.

Fl. Trop. Afr. VI. i. 771 ; For. Fl. Cape, 310.

Chirinda Forest, 3700-4000 ft. Nov. Swyn. 4.

C. Zambesicus, Müll. Arg. (C. Welwitschianus, Müll. Arg.)

Fl. Trop. Afr. VI. i. 758 ; Cat. Afr. Pl. Welw. i. 970.

Victoria Falls, Rogers, 5146; Allen, 418 p.p. ; South of Zambesi,

Engler; Matopos, Rogers, 5252*.

4354-Tannodia Swynnertonii, Prain. (Croton Swynnertonii, S. Moore.)

Fl. Trop. Afr. VI. i. 827 ; Journ. Linn. Soc. Bot. xl. 194 ; see Journ. Bot. 1912, 127.

Chirinda Forest, 3700-4000 ft. Sep.-Dec. Swyn. 109, 6519.

4386-Argomuellera macrophylla, Pax.

Fl. Trop. Afr. VI. i. 925.

Chirinda Forest, 3700-4000 ft. May, Oct., Nov. Swyn. 114.

4395-Neoboutonia Melleri, Prain. (Mallotus Melleri, Müll. Arg.)

Fl. Trop. Afr. VI. i. 922.

North of Lusitu Riv. Swyn. ; Chirinda, Swyn.

4400-Macaranga mellifera, Prain.

Fl. Trop. Afr. VI. i. 943 ; Journ. Linn. Soc. Bot. xl. 201.

Chirinda, 3700-4000 ft. Oct. Swyn. 5 and 2120; Mt. Pene, $7000 \mathrm{ft}$. Oct. Swyn. 6039.

4407-Acalypha Allenii, Hutch.

Fl. Trop. Afr. VI. i. 889; Kew Bull. 1911, 229.

Gwai Forest, Allen, 238; North of Bulawayo, $3400 \mathrm{ft}$. Dec. Eyles, 1130 ; Salisbury, Rand, 1385, 1386 ; Bulawayo, Chubb, 106. 
Genus No.

4407 - A. caperonioides, Baill.

Fl. Trop. Afr. VI. i. 884, as A. peduncularis, Meisn. p.p. see Kew Bull. 1913, 23.

Inyanga, 6000-7000 ft. Cecil, 179, 182 ; between Umtali and Inyanga, Cecil, 167; Salisbury, Cecil, 68; Engler, 3052 ; Rogers, 4003; Gwelo, Jan. Gardner, 33 ; Matopos, $5000 \mathrm{ft}$. Nov. Eyles, 1153 ; Gibbs, 227 ? ; Melsetter, 6000 ft. Oct. Swyn. 2015?; Melsetter, 5000-6000 ft. Oct. Swyn. 2015 ?; North Melsetter, 5000-6000 ft. Dec. Swynn. 6040 ?, 6227?

A. chirindica, S. Moore.

Fl. Trop. Afr. VI. i. 885 ; Journ. Linn. Soc. Bot. xl. 199. Chirinda, 3800 ft. Dec. Swyn. 380, 381.

A. ciliata, Forsk.

Fl. Trop. Afr. VI. i. 901.

Salisbury, Rand, 1388 ; Chirinda, 3700-4000 ft. June, Swyn. 734 ; Victoria, Monro, 946.

A. indica, $L$.

Fl. Trop. Afr. VI. i. 903 ; Cat. Afr. Pl. Welw. i. 978.

Victoria Falls, Rogers, 5386 ; North Melsetter, 2000-6000 ft. Dec. Swyn. 2014.

A. ornata, Hochst.

Fl. Trop. Afr. VI. i. 890 ; Cat. Afr. Pl. Welw. i. 976.

Victoria Falls, Rogers, 5417, 5550.

A. paniculata, Miq.

Fl. Trop. Afr. VI. i. 886; Cat. Afr. Pl. Welw. i. 976.

Melsetter, Johnson, 172.

A. petiolaris, Hochst.

Victoria Falls, April, Flanagan, 3192; Odzani River Valley, Umtali, Teague, 77.

A. punctata, Meisn.

Fl. Trop. Afr. VI. i. 884, as A. peduncularis, Meisn. p.p. see Kew Bull. 1913, 23.

Upper Buzi, 3000 ft. Swyn. 383 ; Sep. Swyn. 739 ?

A. segetalis, Müll. Arg.

Fl. Trop. Afr. VI. i. 904 ; Cat. Afr. Pl. Welw. i. 979.

Victoria Falls, Rogers, 5386a.

A. senensis, Klotzsch. (A. zambesica, Müll. Arg.)

Fl. Trop. Afr. VI. i. 888.

Matopos, Oct. Gibbs, 225 ; Victoria Falls, Allen, 197 ; Bulawayo, Jan. Gardner, 56 ; Chubb, 353 ; Chirinda, Swyn. 382. 
Genus No.

A. villicaulis, Hochst.

Fl. Trop. Afr. VI. i. 893.

Salisbury, Engler; Rand, 1384; Bulawayo, Oct.-Dec. Chubb, 369 ; Chirinda, 3500-4000 ft. April, Swyn. 382, 383 ; Upper Buzi, 3000 ft. Dec. Swyn. 1153.

\section{A. spp.}

North of Bulawayo, $3400 \mathrm{ft}$. Dec. Eyles, 1120.

South Rhodesia, Rand, 204, 205.

4416-Tragia dioica, Sond.

Fl. Trop. Afr. VI. i. 993.

Bulawayo, Kolbe, in Herb. Bolus, 4109.

T. Gardneri, Prain.

Fl. Trop. Afr. VI. i. 994 ; Kew Bull. 1909, 52.

Gwelo, Jan. Gardner, 34.

T. kirkiana, Müll. Arg.

Fl. Trop. Afr. VI. i. 998.

Bulawayo, Rogers, 5915 ; Victoria Falls, Rogers, 5594. ${ }^{\mathrm{I}}$

T. natalensis, Sond. (T. ambigua, S. Moore, and var. urticans, S. Moore.)

Fl. Trop. Afr. VI. i. 974 ; Journ. Linn. Soc. Bot. xl. 202.

Chirinda Forest, 3700-4000 ft. June, Swyn. 795; May, Swyn. 446.

T. Okanyua, Pax. (T. angustifolia, Pax.)

Fl. Trop. Afr. VI. i. 986.

Bulawayo, Jan. Gardner, 55; Chubb, 66 ; Victoria Falls, Rogers, $5594 \%$.

T. rhodesiae, Pax.

Fl. Trop. Afr. VI. i. 979.

Salisbury, Sep. Engler, 3073; Rand, 644, 1387 ; Marshall.

4424-Ricinus communis, $L$.

Fl. Trop. Afr. VI. i. 945 ; Cat. Afr. Pl. Welw. i. 983.

South Rhodesia, Rhod. Agric. Journ. 1904, 135. Naturalized.

Odzani River Valley, Umtali, Teague, 269.

4436-Hevea sp.

Matopos, Govt. Herb. 901, cultivated.

4444-Manihot Glaziovii, Miill. Arg.

Fl. Trop. Afr. VI. i. 839.

Salisbury, Govt. Herb. 765, cultivated.

4448-Cluytia inyangensis, Hutch.

Fl. Trop. Afr. VI. i. 804.

Inyanga, 6000-7000 ft. Cecil, 181.

r Rogers had this number named T. okanyua, Pax, by Mr. Spencer Moore. 
Genus No.

C. monticola, S. Moore.

Fl. Trop. Afr. VI. i. 803 ; Journ. Linn. Soc. Bot. xl. 197.

Mt. Pene, 6500-7000 ft. Sep.-Oct. Swyn. 2012, 6159.

C. Paxii, Knauf. (C. phyllanthoides, S. Moore.)

Fl. Trop. Afr. VI. i. 809 ; Journ. Linn. Soc. Bot. xl. 198.

Melsetter, 6000 ft. Sep. Swyn. 1120, 1722.

C. robusta, $P a x$.

Fl. Trop. Afr. VI. i. 811.

Melsetter, Swyn. 1530 ; Mt. Pene, Swyn. 6042 ; Chirinda, Swyn. $1531 a$.

C. stelleroides, S. Moore.

Fl. Trop. Afr. VI. i. 804 ; Journ. Linn. Soc. Bot. xl. 198.

North Melsetter, 5000-6000 ft. Oct. Swyn. 6214.

C. Swynnertonii, S. Moore.

Fl. Trop. Afr. VI. i. 811 ; Journ. Linn. Soc. Bot. xl. 197.

Chipete and Chirinda, 3700-4000 ft. May-June, Swyn. 197, 530a, $530 b, 530 c$.

C. yolubilis, Hutch.

Fl. Trop. Afr. VI. i. 809.

Chimanimani, Johnson, 188.

4463-Ricinodendron Rautanenii, Schinz.

Fl. Trop. Afr. VI. i. 746.

Victoria Falls, Kirk; Allen, 191.

4464-Gelonium procerum, Prain.

Fl. Trop. Afr. VI. i. 948 ; Journ. Linn. Soc. Bot. xl. 201.

Chirinda Forest, 3700-4000 ft. Oct. Swyn. 104; Chimanimani Mts. 7000 ft. Swyn. 1115.

4478-Excœcaria sp.

Gwanda, Noble, 23.

4483-Sapium madagascariense, Prain. (Exccecaria sylvestris, S. Moore.)

Fl. Trop. Afr. VI. i. 1010 ; Journ. Linn. Soc. Bot. xl. 204.

Chirinda Forest, 3700-4000 ft. Oct. Jan. Swyn. 72, 72a.

S. Mannianum, Benth.

Fl. Trop. Afr. VI. i. 1016; Cat. Afr. Pl. Welw. i. 986.

Chipete Forest, 3800 ft. March, Swyn. 103; Chirinda, 3800 ft.

Sep. Swyn. 741.

4498-Euphorbia abyssinica, Gmelin.

Fl. Trop. Afr. VI. i. 588.

South Rhodesia, Rand.

E. angularis, Klotzsch.

Fl. Trop. Afr. VI. i. 584.

Matopos, Nov. Marloth, 3418. 
A Record of Plants Collected in Southern Rhodesia.

Genus No.

4498-E. crotonoides, Boiss.

Fl. Trop. Afr. VI. i. 518.

Redbank, Flanagan, 3186.

E. cyparissioides, Pax. (E. ericoides, Lam.)

Fl. Trop. Afr. VI. i. 542 ; Cat. Afr. Pl. Welw. i. 951.

Rusapi, Engler, 3112 ; North Melsetter, Swyn. 6217.

var. minor, N. E. Br.

Fl. Trop. Afr. VI. i. 542.

North Melsetter, 6000 ft. Sep. Swyn. 6043, 6044; Haroni Riv. 5000 ft. Oct. Swyn. 1529 ; Macheke, Rogers, 4027.

E. depauperata, Hochst.

Fl. Trop. Afr. VI. i. 537.

Mt. Pene, 7000 ft. Sep. Swyn. 6041 ; Haroni Riv. 5500 ft. Oct. Swyn. 1528.

E. espinosa, $P a x$.

Fl. Trop. Afr. VI. i. 547.

Bulawayo, Chubb, 345 ; Victoria, Monro, 319.

E. Eylesii, Rendle.

Fl. Trop. Afr. VI. i. 512 ; Journ. Bot. 1905, 52.

Deka, 2400 ft. May, Eyles, 130 ; Kesi, April, Flanagan, 3284 ;

Victoria, Monro, 910 ; Victoria Falls, Rogers, 5559*, 7138*.

E. grandidens, Haw.

For. Fl. Port. E. Afr. 105 ; For. Fl. Cape, 317.

Matopos, Davy.

E. griseola, $P a x$, var. robusta, $P a x$.

Fl. Trop. Afr. VI. i. 578.

Matopos, 5000 ft., Engler, $2860 b$.

E. helioscopia, $L$.

Bulawayo, 4500 ft. Jan. Eyles, 62.

E. livida, E. Mey.?

Victoria Falls, Galpin, 7351.

E. matabelensis, Pax. (E. jageriana, Pax, E. trifurca, N. E. Br.) Fl. Trop. Afr. VI. i. 546.

Matopos, Sep. Gibbs, 24 ; Engler ; Victoria Falls, Galpin, 7056 ; Rogers, 5306; Victoria, Monro, 424; between Umtali and Melsetter and other parts of Mashonaland, Swyn. 691, 6611; Matabeleland, Penther, 944; Chirinda, 3500 ft. Oct. Swyn. 691.

E. mozambicensis, N. E. Br.

Fl. Trop. Afr. VI. i. 509.

Victoria Falls, Rogers, 7002*. 
E. Oatesii, Rolfe.

Fl. Trop. Afr. VI. i. 522.

Matabeleland, Oates; Gwelo, Rand, 218.

E. Reinhardtii, Volk.

Fl. Trop. Afr. VI. i. 590.

Bulawayo, Nov. Marloth, 3417 ; Matopos, Davy; Engler.

E. rubriflora, $N$. E. Br.

Fl. Trop. Afr. VI. i. 509.

Victoria Falls, Rogers, 5565 ; Allen.

E. Schimperiana, Scheele.

Fl. Trop. Afr. VI. i. 533.

Chirinda, 3700-4000 ft. Swyn. 1531.

var. Buchanani, N. E. Br.

Fl. Trop. Afr. VI. i. 534.

Chirinda Forest, 3800 ft. Sep. Swyn. 238.

E. Schinzii, $P a x$.

Fl. Trop. Afr. VI. i. 567.

Bulawayo, 4500 ft. Eyles, 1247 ; Rand, 396.

E. tettensis, Klotzsch.

Fl. Trop. Afr. VI. i. 494.

Igusi, April, Flanagan, 3191.

E. tortistyla, N. E. Br.

Fl. Trop. Afr. VI. i. 569.

Victoria, Monro, 490.

E. trichadenia, Pax. (E. benguellensis, Pax.)

Fl. Trop. Afr. VI. i. 523.

Matopos, Oct. Gibbs, 234 ; Victoria, Monro, 141.

E. whyteana, Baker $f$.

Fl. Trop. Afr. VI. i. 540.

Chimanimani Mts. 7000 ft. Sep. Swyn. 1526.

E. spp.

Gwai Forest, Allen, 264.

Mazoe, 4400 ft. April, Eyles, 314, cf. E. Eylesii, Rendle.

Mazoe, 4400 ft. April, Eyles, 365.

Victoria Falls, April, Flanagan, 3246.

South Rhodesia, Rand, 395, 397.

4500-Synadenium gazenae, N. E. Br.

Fl. Trop. Afr. VI. i. 467 ; Journ. Linn. Soc. Bot. xl. 190.

Chipetzana Riv. 3000 ft. April, Swyn. 1505. 


\section{SERIES SAPINDALES.}

\section{FAMily CLIII.-ANACARDIACEAE, Lindl.}

Genus No.

4558-Sclerocarya caffra, Sond.

Fl. Trop. Afr. i. 449 ; Fl. Cap. i. 525 ; For. Fl. Port. E. Afr. 39 ; Cat. Afr. Pl. Welw. i. 176 ; Harv. Gen. S.A. Pl. 64 ; Nat. Pl. 307.

Matopos, Davy; Oct. Gibbs, 261; Bulawayo, Engler; Chubb, 321 ; Gwanda, Noble, 10 ; Umtali, Govt. Herb. 843; Sabi Riv. fr. Nov. Swyn. 1210 ; Melsetter, Swyn.; Victoria, Monro, 531.

4563-Lannea discolor (Sond.), Engl. (Odina discolor, Sond.)

Fl. Cap. i. 504.

Umtali, Engler; Salisbury, Engler.

L. edulis (Sond.), Engl. (Odina edulis, Sond.)

Fl. Cap. i. 503.

Matopos, fl. Sep. fr. Oct. Gibbs, 71; Salisbury, Darling; Chirinda, 3800 ft. fl. \& fr. Oct. Swyn. 441; South Melsetter, Swyn.; Charter, Govt. Herb. 3017; Victoria, Monro, 523; North of Bulawayo, Engler.

var. integrifolia, Engl.

Salisbury, Engler.

L. Schimperi (Hochst.), Engl. (Odina Schimperi, Hochst.)

Fl. Trop. Afr. i. 445.

Matopos, Oct. đ Gibbs, 268.

L. Stuhlmanni (Engl.), Torre \& Harms. (Odina Stuhlmanni, Engl.) Sabi Riv. 1000 ft. Nov. Swyn. 1205.

L. Wodier, Roxb. var. brevifolia, Engl.

Victoria, Monro, 745.

\section{L. spp.}

Victoria, Monro, 552; 587, 641.

Mazoe, 4300-4800 ft. Sep. Eyles, 422.

Bulawayo, Oct.-Dec. Chubb, 306.

4589-Heeria insignis, O. Kuntze, var. reticulata, Baker $f$.

Journ. Bot. 1899, 428.

Bulawayo, Jan. Rand, 64; fr. May, Rand, 307 ; between Mt.

Pene and Haroni Riv. 6000 ft. fr. Sep. Swyn. 680.

H. paniculosa, Engl.

Matopos, Rogers, 5412. 
Genus No.

H. pulcherrima (Schweinf.), O. Kuntze. (Rhus pulcherrima, Oliv. Fl. Trop. Afr. i. 436.

Matopos, Engler; Salisbury, Rogers, 5769 ; Charter, Govt. Herb. 3016.

H. reticulata (Baker), Engl.

Salisbury, Engler; Victoria, Monro, 695; Matopos, Rogers, 5363.

\section{H. spp.}

Victoria, Monro, 332, 437.

4594-Rhus bulawayensis, Diels.

Bulawayo, Sep. Engler, 2923.

R. chirindensis, Baker $f$.

Journ. Linn. Soc. Bot. xl. 49.

Chirinda, 3800 ft. Swyn. 168.

R. erosa, Thunb.

Fl. Cap. i. 516 ; For. Fl. Cape, 194.

Victoria, Monro, 307.

R. lævigata, $L$.

Fl. Cap. i. 514 ; For. Fl. Cape, 195.

Victoria, Monro, 739.

R. lancea, $L . f$.

Fl. Cap. i. 514 ; For. Fl. Cape, 194.

Bulawayo, July, Rand, 444; May, Rand, 302, 304 ; Chubb, 41, 45, 51, 315 ; Matopos, Sep. Galpin, 7066 ; Davy ; fl. \& fr. Sep. Gibbs, 1; Gwanda, Noble, 36.

\section{R. lanceolata?}

Matopos, Govt. Herb. 895 ; Salisbury, Govt. Herb. 937.

R. leptodictya, Diels.

Bulawayo, Sep. Engler, 2915.

R. lucida, $L$.

Fl. Cap. i. 517.

Chimanimani Forest, 6500 ft. fl. \& young fr. Sep. Swyn. 635.

R. mucronifolia, Sond. (R. salicifolia, Presl.)

Fl. Cap. i. 521; For. Fl. Cape, 193.

Gwanda, Noble, 65 ; Mazoe, Govt. Herb. 2089 ?

R. paniculosa, Sond. (Anaphrenium paniculosum, Engl.)

Fl. Cap. i. 522.

Bulawayo, 4500 ft. Dec. Eyles, 13; Rogers, 5508; Matopos, March, Flanagan, 2945 ; Rogers, 5413, 5639.

R. rehmannia, Engl.

Bulawayo, Chubb, $4 a$.

R. Sonderi, Engl.

Chirinda, 3500 ft. fl. \& fr. Oct. Swyn. 174; Chipete Forest, 3800 ft. Oct. Swyn. $174 a$. 
Genus No.

var. pilosa, Engl.

Melsetter, $6000 \mathrm{ft}$. Swyn. 6165.

R. tenuinervis, Engl.

Cat. Afr. Pl. Welw. i. 182.

Bulawayo, May, Rand, 306.

R. tomentosa, $L$.

Fl. Cap. i. 508; For. Fl. Cape, 195.

Melsetter, $6000 \mathrm{ft}$. Sep. Swyn. 667.

R. trifoliolata, Baker $f$.

Journ. Bot. 1899, 429.

Bulawayo, Dec. Rand, 66.

R. villosa, $L . f$.

Fl. Trop. Afr. i. 439 ; Fl. Cap. i. 510 ; Cat. Afr. Pl. Welw. i. 182 ;

For. Fl. Cape, 196.

Bulawayo, Jan. Rand, 65 ; Rogers, 5506; Matopos, Oct. Gibbs, 247 ; Engler; Mazoe, 4600 ft. Aug. Eyles, 398; Salisbury, Engler; Victoria, Monro, 1004.

R. Welwitschii, Engl. var. angustifolia, Baker $f$.

Journ. Bot. 1899, 429.

Bulawayo, Jan. Rand, 97.

R. spp.

Victoria Falls, fr. April, Flanagan, 3211.

Victoria, Monro, 314.

Salisbury, Rogers, 5574.

Lomagundi, Govt. Herb. 985.

Umvukwe Hills, Govt. Herb. 1090.

Mazoe, Govt. Herb. 2082.

South Rhodesia, Rand, 303, 305.

Family CLVII.-AQUIFOLIACEAE, DC.

4614-Ilex capensis, Sond. \& Harv. (I. mitis, Radl.)

Fl. Trop. Afr. i. 359 ; Fl. Cap. i. 473 ; For. Fl. Port. E. Afr. 22 ;

For. Fl. Cape, 151 ; Cat. Afr. Pl. Welw. i. 143.

Mt. Pene, 7000 ft. Oct. Swyn. 6210 ; Mazoe, 4300 ft. Sep. Eyles, 436.

Family CLVIII.-CELASTRACEAE, Lindl.

4625-Celastrus concinnus, N. E. Br.

Kew Bull. 1906, 16.

Chirinda, 3700-4000 ft. fl. \& fr. Oct. Swyn. 118. 
C. mossambicensis, Klotzsch.

Fl. Trop. Afr. i. 362.

Chikore Hills, 3500 ft. April, Swyn. 185.

C. polyacanthus, Sond.

Fl. Cap. i. 455.

Victoria, Monro, 1100.

4627-Gymnosporia buxifolia (Sond.), Szyszyl. (Celastrus buxifolius, L. Fl. Cap. i. 459 ; Nat. Pl. 535 ; For. Fl. Cape, 185.

Matopos, Engler; Victoria, Monro, 1023 ; Bulawayo, Rogers 13520*.

G. senegalensis, Loes. (Celastrus senegalensis, Lam.)

Fl. Trop. Afr. i. 361 ; Cat. Afr. Pl. Welw. i. 145.

Matopos, Engler; fr. Sep. Galpin, 7077 ; Rogers, 5244 ; Victoria Falls, Engler; Rogers, 7420 ; Victoria, Monro, 382 ; Upper Buzi, 3500 ft. May, Swyn. 2069.

var. inermis?.

Bulawayo, May, Rand, 298 ; Victoria Falls, July, Allen, 411. var. spinosa?.

Bulawayo, May, Rand, 413; Mazoe, 4800 ft., June, Eyles, 374.

\section{G. spp.}

Victoria, Monro, 386, 738a.

South Rhodesia, Rand, 324, 442.

4629-Catha edulis, Forsk.

Fl. Trop. Afr. i. 365; For. Fl. Cape, 181; Harv. Gen. S.A. Pl. 53.

Salisbury, fr. July, Rand, 567 ; Chipete, 3800 ft. April, Swyn. 102 ; Chirinda Forest, 3700-4000 ft. May, Swyn. 102? Chirinda, 3500 ft. fr. Oct. Swyn. 1342 ; Odzani River Valley, Umtali, Teague, 112.

4630-Pterocelastrus rostratus, Walp.

Fl. Cap. i. 463 ; For. Fl. Port. E. Afr. 36 ; For. Fl. Cape, 187.

Mt. Pene Forest, 6500 ft. Sep. Swyn. 1301 ; Chimanimani Mts. 7000 ft. Sep. Swyn. 1302.

4640--Elæodendron capense, $E$. \& $Z$.

Fl. Cap. i. 468 ; For. Fl. Cape, 190.

Chikore, 3600 ft. March, Swyn. 167; Chirinda, 3700-4000 ft. Oct. Swyn. 1174.

E. matabelicum, Loes.

Matopos, Sep. Engler, 2835 ; Oct. Gibbs, 365 ; Victoria, Monro, $738,844$.

E. sp.

South Rhodesia, Rand, 427. 
Genus No.

Family CLIX.-HIPPOCRATEACEAE, H. $B$. \& $K$.

4661-Hippocratea Buchanani, Loes.

Victoria Falls, Rogers, 5371.

H. cymosa, De Wild. \& Th. Dur.

Victoria Falls, Engler.

H. obtusifolia, Roxb.

Fl. Trop. Afr. i. 369 ; Cat. Afr. Pl. Welw. i. 149.

Victoria Falls, Sep. Galpin, 7017; Sep. Gibbs, 139 ; Rogers, 5785 ; Sabi Riv. 1000 ft. Nov. Swyn. 1200; fr. Nov. Swyn. 1203.

Family CLXII.-ICACINACEAE, Miers.

4686-Apodytes dimidiata, E. Mey.

Fl. Trop. Afr. i. 355 ; Fl. Cap. i. 235 ; Cat. Afr. Pl. Welw. i. 142 ; For. Fl. Port. E. Afr. 31 ; For. Fl. Cape, 135.

Chirinda, 3800 ft. Swyn. 184.

FAmily CLXV.-SAPINDACEAE, Juss.

4724-Paullinia pinnata, $L$.

Fl. Trop. Afr. i. 419 ; For. Fl. Port. E. Afr. 31 ; Cat. Afr. Pl. Welw. i. 166.

Victoria Falls, Sep. Galpin, 7018; fl. \& fr. Sep. Gibbs, 124 ; Engler; Rogers, 5101, 8674; Matopos, March, Flanagan, 2967.

4726-Cardiospermum corindum, L. (C. canescens, Wall.)

Fl. Trop. Afr. i. 418 ; Cat. Afr. Pl. Welw. i. 167.

Matopos, fl. \& fr. Sep.-Oct. Gibbs, 78; Engler; Rogers, 5199.

C. Halicacabum, $L$.

Fl. Trop. Afr. i. 417 ; Cat. Afr. Pl. Welw. i. 167.

Bulawayo, Rogers.

4734-Allophylus alnifolius (Baker), Radlk. (Schmidelia alnifolia, Baker.)

Fl. Trop. Afr. i. 422.

Matopos, Engler.

A. chirindensis, Baker $f$.

Journ. Linn. Soc. Bot. xl. 48.

Chirinda Forest, 3700-4000 ft. Swyn. 112.

A. rubrifolius (Hochst.), Torre \& Harms. (Schmidelia rubrifolia, Hochst.)

Fl. Trop. Afr. i. 423.

Victoria Falls, Allen, 143 ; Rogers, 5538. 
Genus No.

4784 -Pappea capensis, E. \& Z. (Sapindus Pappea, Sond.)

Fl. Cap. i. 241 ; For. Fl. Port. E. Afr. 33 ; For. Fl. Cape, 171.

Bulawayo, Galpin, 7064 ; Matabeleland, Davy.

P. fulva, Conrad.

Victoria, Monro, 947 ; Bulawayo, Chubb, 44 ; Salisbury, Rogers, 4077.

\section{P. spp.}

Viotoria, Monro, 944, 988.

4831-Dodonæa viscosa, $L$.

Fl. Trop. Afr. i. 433 ; Fl. Cap. i. 242.

Victoria, Monro, 576; Melsetter and Nyahodi Riv. Swyn.; Odzani River Valley, Umtali, Teague, 86.

\section{Family CLXVII.-MELIANTHACEAE, Endl.}

4853-Bersama maschonensis, Gürke.

Umtali, Sep. Engler, 3141.

B. Swynnertoni, Baker $f$.

Journ. Bot. 1907, 14.

Melsetter, 3800 ft. Swyn. 9 ; Chipete Forest, Dec. Swyn.

\section{Family CLXVIII.-BALSAMINACEAE, Lindl.}

4856-Impatiens Cecili, N. E. Br.

Kew Bull. 1906, 101.

Manica Dist. Cecil, 169 ; Melsetter, 6000 ft. Sep. Swyn. 618.

I. walleriana, Hook. $f$.

Fl. Trop. Afr. i. 302.

Chirinda Forest, 3700 ft. April, Swyn. 344.

I. zombensis, Baker, forma.

Chirinda Forest, 3700-4000 ft. April, Swyn. 343

\section{SERIES RHAIMNALES.}

FAmily CLXIX.-RHAMNACEAE, Lindl.

\section{1-Zizyphus espinosus, Buettn.}

South Rhodesia, Rand, 301.

Z. jujuba, Lam.

Fl. Trop. Afr. i. 379 ; For. Fl. Port. E. Afr. 35.

Mazoe, 4300 ft. Sep. Eyles, 407 ; Victoria Falls, Rogers, 5726. 
Genus No.

var. nanus, Engl.

Salisbury, Engler.

Z. mucronata, Willd.

Fl. Trop. Afr. i. 380 ; Fl. Cap. i. 475 ; Nat. Pl. 47 ; For. Fl. Port. E. Afr. 35 ; For. Fl. Cape, 177.

Bulawayo, Dec. Rand, 9; Chubb, 27 ; Rogers, 5505; Nov. Eyles, 1209 ; Govt. Herb. 915 ; Matopos, Davy ; Govt. Herb. 905; Gwanda, Noble, 30 ; Umtali, Govt. Herb. 836 ; Victoria, Monro, 985; Gambadzia Riv. 3300 ft. fr. May, Swyn. 1385; Umvumvumvu Riv. 4000 ft. fr. April, Swyn. 2070; South Melsetter, Swyn.; Salisbury, Rogers, 5779; Matabeleland, Marloth.

\section{Z. spp.}

Bulawayo, Chubb, 396.

Victoria, Monro, 779.

South Rhodesia, Rand, 589.

4868-Berchemia discolor, Hemsl.

Fl. Trop. Afr. i. 381 ; For. Fl. Port. E. Afr. 35.

Sabi Riv. 1000 ft. Nov. Swyn. 1215; Hartley, Govt. Herb. 2095. 4875-Rhamnus prinoides, L'Hérit.

Fl. Trop. Afr. i. 382 ; Fl. Cap. i. 477 ; For. Fl. Cape, 179.

Chipete, 3800 ft. fr. May, Swyn. 428.

R. sp.

Bulawayo, 4500 ft. Dec. Eyles, 1005.

4886--Phylica paniculata, Willd.

Fl. Cap. i. 482 ; For. Fl. Cape, 180.

Chimanimani Mts. 7000 ft. fr. Sep. Swyn. 632a.

4888-Lasiodiscus Holtzii, Engl.

Chirinda Forest, 3700-4000 ft. fl. \& fr. Oct. Swyn. 121 ; young fr. Dec. Swyn. 6227.

4902-Gouania longispicata, Engl.

Chirinda Forest, 3700-4000 ft. April, Swyn. 96.

4905-Helinus mystacinus, Hemsl.

Fl. Trop. Afr. i. 385.

South Rhodesia, Rand, 36.

H. mystacinus, E. Mey.

Chirinda, 3700-4000 ft. fl. \& fr. May, Swyn. 217.

H. ovatus, E. Mey.

Fl. Trop. Afr. i. 384 ; Fl. Cap. i. 479 ; Nat. Pl. 44 ; Cat. Afr. Pl. Welw. i. 151.

Matopos, 4500 ft. Nov. Eyles, 1185; Rogers, 5646; Victoria, Monro, 1067 ; Victoria Falls, Rogers, 7146. 
FAmily CLXX.-VITACEAE, Lindl.

Genus No.

4909 -Vitis spp.

Victoria, Monro, 1026.

Victoria Falls, Rogers, 5062, 5070.

4910_Ampelocissus Grantii, Planch.

Victoria Falls, Rogers, 5541.

A. mossambicensis, Planch.

South Melsetter, Swyn.

A. obtusata, Planch. (Vitis obtusata, Welw.)

Fl. Trop. Afr. i. 414 ; Cat. Afr. Pl. Welw. i. 156.

Salisbury, Rand, 1362 .

4917-Rhoicissus capensis, Planch.

Chirinda, 3700-4000 ft. Nov. Swyn. 1370 ; Chikore Hills, 37004000 ft. bud Nov. Swyn. 1376.

R. cunefolia, Planch.

Chirinda, 3800 ft. bud Nov. Swyn. 221.

R. erythrodes, Planch. (Vitis erythrodes, Fresen.)

Fl. Trop. Afr. i. 401 ; Cat. Afr. Pl. Welw. i. 157.

Salisbury, July, Rand, 443.

var. ferruginea (Baker), Planch.

Mazoe, 4700 ft. Jan. Eyles, 507.

R. rhomboidea, Planch.

Mt. Pene Forest, 6500-7000 ft. Swyn. 1382 ; Chimanimani Mts. 7000 ft. Swyn. 1381; Chirinda Forest, 3700 ft. fr. Oct. Swyn. 92.

R. sansibarensis, Gilg.

Lusitu Riv. 3000 ft. fr. April, Swyn. 1380.

R. spp.

Victoria, Monro, 1060, ef. R. sansibarensis, Gilg. ; 1080, ef. R. sericea, E. \& Z.; and 577.

Umvumvumvu Riv. Swyn. 1379, ef. R. sansibarensis, Gilg.

4918-Cissus Buchanani, Planch.

Cat. Afr. Pl. Welw. i. 164.

Chipete, 3800 ft. fr. March, Swyn. 231.

C. congesta, Planch.

Salisbury, Rogers, 5775 .

C. crotalarioides, Planch.

Salisbury, Rand, 1339, 1340.

C. cymosa, Schum. (Vitis Thonningii, Baker.)

Fl. Trop. Afr. i. 407.

Salisbury, Sep. Rand, 606. 
C. fragilis, E. Mey.

Fl. Cap. i. 249.

Victoria Falls, Rogers, 5572, 5614.

C. gracilis, Guill. \& Perr.

Victoria, Monro, 1043 ; Victoria Falls, Rogers, 5552.

C. hypargyrea, Gilg.

Salisbury, Rand, 1338.

C. jatrophoides, Planch. (Vitis jatrophoides, Welw.)

Fl. Trop. Afr. i. 400 ; Cat. Afr. Pl. Welw. i. 161.

Salisbury, Rand, 1337.

C. Marlothii, Gilg.

Matopos, Nov. Märloth, 3386.

C. rhodesiae, Gilg.

Salisbury, Engler.

C. rotundifolia, Vahl.

Sabi Riv. 1000 ft. bud Nov. Swyn. 2071.

C. zombensis, Gilg. \& Brandt, forma.

Chirinda, 3500 ft. fr. Sep. Swyn. 194.

C. spp.

Bulawayo, 4500 ft. Feb. Eyles, 101, cf. C. Sandersoni, Harv.

Salisbury, Rand, 1336, 1363.

Victoria, Monro, 519.

Victoria Falls, Galpin, 7380.

Mazoe, 4400 ft. Jan. Eyles, 504; 4800 ft. Nov. Eyles, 459.

\section{SERIES MALVALES.}

\section{Family CLXXIV.-TILIACEAE, Juss.}

\section{3-Corchorus asplenifolius, Burch.}

Fl. Cap. i. 229.

Bulawayo, Dec. Gardner, 97; Rogers, 5743, 5495; Wankie, Rogers, 6012.

C. hirsutus, $L$.

Fl. Trop. Afr. i. 264.

Matopos, Nov. Eyles, 1150 ; fl. and fr. Oct. Gibbs, 237 ; Engler ;

March, Flanagan, 2978 ; Rogers, 5650 ; Victoria, Monro, 1066.

C. Kirkii, N. E. Br.

Matopos, Rogers, 5345, 5349.

C. mucilagineus, Gibbs.

Journ. Linn. Soc. Bot. xxxvii. 433.

Matopos, Sep. Gibbs, 8. 
Genus No.

C. muricatus, Hochst.

Fl. Trop. Afr. i. 263.

Victoria Falls, Allen, 87.

C. serræfolius, Burch.

Fl. Cap. i. 229.

Bulawayo, Dec. Rand, 8.

C. tridens, $L$.

Fl. Trop. Afr. i. 264 ; Cat. Afr. Pl. Welw. i. 101.

Victoria Falls, July, Kolbe, 3174 ; Rogers, 5537 ; Ngomi, April, Flanagan, 3290.

C. trilocularis, $L$.

Fl. Trop. Afr. i. 262 ; Fl. Cap. i. 229 ; Cat. Afr. Pl. Welw. i. 100.

Bulawayo, 4500 ft. Dec. Eyles, 5 ; Victoria, Monro, 974 ; Chipete Forest, 3800 ft. fl. \& fr. May, Swyn. 469 ; Upper Buzi, 3800 ft. fr. April, Swyn. 1480 ; Chipetzana Riv. 3000 ft. fr. April, Swyn. 1481.

C. sp.

Gwelo, Jan. Gardner, 40, cf. C. trilocularis, L.

4957-Sparmannia palmata, E. Mey., forma.

Fl. Cap. i. 224.

Chipete Forest, 3800 ft. April, Swyn. 225 ; Melsetter, $6000 \mathrm{ft}$. fr. April, Swyn. 789 ; Chirinda, Swyn.

4966-Grewia cana, Sond.

Fl. Cap. i. 225.

Bulawayo, 4500 ft. Oct. Eyles, 1089 ; Monro, 55; Khami, Nov. Marloth, 3394.

G. chirindae, Baker $f$.

Journ. Linn. Soc. Bot. xl. 31.

Chirinda, 3700-4000 ft. Nov. Swyn. 131 ; Chipete, Swyn.

G. flaya, $D C$.

Fl. Trop. Afr. i. 250 ; Fl. Cap. i. 225 ; For. Fl. Cape, 148.

Matopos, Oct. Gibbs, 226 ; Engler ; Victoria Falls, Rogers, 5144.

G. flavescens, Juss.

Victoria Falls, Rogers, 5597.

G. guazumifolia, Juss.

Fl. Trop. Afr. i. 245.

Victoria Falls, Rogers, 5590.

G. monticola, Sond.

Fl. Cap. i. 226.

Bulawayo, 4500 ft. Nov. Eyles, 1225; Chubb, 357; Rogers, 5498 ; Victoria, Monro, 667.

G. obovata, K. Schum.

Sabi Riv. 1000 ft. fr. Nov. Swyn. 1217. 
A Record of Plants Collected in Southern Rhodesia.

Genus No. G. occidentalis, $L$.

Fl. Trop. Afr. i. 246 ; Fl. Cap. i. 225 ; For. Fl. Cape, 147 ; Nat. Pl. 210.

Gwai Forest, 3800 ft. Allen, 236 ; Chirinda, 3800 ft. Dec. Swyn. 6628.

G. pilosa, Lam.

Fl. Trop. Afr. i. 250 ; Cat. Afr. Pl. Welw. i. 96.

Plumtree, fr. April, Flanagan, 3207 ? ; Victoria Falls, Rogers, 5591.

\section{G. spp.}

Bulawayo, Dec. Gardner, 92, cf. G. subspathulata, N. E. Br.; Monro, 67, 81 ; Eyles, 1010.

Victoria Falls, Allen, 81, cf. G. salvifolia, Heyne ; Allen, 67 ; Allen, 150, ef. G. occidentalis, L. ; Rogers, 7265, ef. G. cana, Sond.

Mazoe, 4300 ft. Dec. Eyles, 208.

Victoria, Monro, 361, 740, 794, 919, 700.

South Rhodesia, Rand, 40, cf. G. salvifolia, Heyne; Rand, 11 and 294.

4975-Triumfetta angolensis, Sprague \& Hutchinson.

Journ. Linn. Soc. Bot. xxxix. 256 (1909).

Odzani River Valley, Umtali, Teague, 204.

T. annua, $L$.

Fl. Trop. Afr. i. 256 ; Cat. Afr. Pl. Welw. i. 97.

Mazoe, 5000 ft. March, Eyles, 255; Victoria Falls, April, Flanagan, 3236 ; Chirinda, 3800 ft. fl. \& fr. April, Swyn. 272;

fl. May, Swyn. 473.

T. annuletum, Sprague.

Gwelo, Sr. Phil. 39 ; Umtali, 3900 ft. Engler, 3121a.

T. dekindtiana, Engl.

Umvumvumvu Riv. 4000 ft. fr. April, Swyn. 2062.

T. diversifolia, E. Mey.

Victoria, Monro, 886.

T. effusa, E. Mey.

Fl. Cap. i. 228 ; Nat. Pl. 318 (as T. pilosa, Roth.).

Victoria Falls, April, Flanagan, 3171 ; Chirinda, 3800 ft. fr. April, Swyn. 1156 ; Victoria, Monro, 902.

T. Mastersii, Baker $f$.

Salisbury, Aug. Rand, 440 ; fl. \& young fr. Sep. Rand, 592 ; Bulawayo, Rogers, 5472 ?; Chipetzana Riv. 3000 ft. bud Oct. Swyn. 2061 ; Nyahodi Riv. 5000 ft. bud Sep. Swyn. 2060; Matabeleland, Oates (as T. Welwitschii, Mast.), South Rhodesia, Rand, 81. 
T. pilosa, Roth.

Fl. Trop. Afr. i. 257 ; Fl. Cap. i. 227 ; Cat. Afr. Pl. Welw. i. 98. Chirinda, 3500 ft. Feb. Swyn. 271; Melsetter, W. H. Johnson, 120.

\section{T. rhomboidea, Jacq.}

Fl. Trop. Afr. i. 257 ; Fl. Cap. i. 227 ; Cat. Afr. Pl. Welw. i. 98 ; Nat. Pl. 252 (as T. effusa, E. Mey.).

Matopos, March, Flanagan, 2988; Gwelo, Sr. Phil. 23 ; Chirinda, 3800 ft. Feb. Swyn. 270; fr. May, Swyn. 472; April, Swyn. 1157 ; Upper Buzi, 3000 ft. fr. April, Swyn. 2063.

T. Sonderi, Fic. \& Hiern.

Victoria Falls, Rogers, 5707.

T. trichocarpa, Sond. (? See note Fl. Trop. Afr. i. 260.)

Fl. Cap. i. 228.

Victoria Falls, Rogers, 7030, 7021. ${ }^{\mathrm{x}}$

T. Welwitschii, Mast.

Fl. Trop. Afr. i. 255 ; Cat. Afr. Pl. Welw. i. 97.

Matopos, fl. and fr. Oct. Gibbs, 261 ; Mazoe, 4300 ft. Sep. Eyles, 426 ; Matopos, Engler; Salisbury, May, Flanagan, 3230; Rand, 1341; Odzani River Valley, Umtali, Teague, 266.

var. laxiflora, Sprague \& Hutch. (T. laxiflora, Engl.)

Salisbury, $4800 \mathrm{ft}$. Engler, 3025.

var. Rehmannii, Sprague \& Hutch.

Rusapi, 4200 ft. Engler, 3116.

\section{T. spp.}

Salisbury, May, Flanagan, 3197, cf. T. semitriloba, L.

Victoria, Monro, 502, 589.

Bulawayo, 4500 ft. Sep. Eyles, 1078.

\section{FAMiLy CLXXV.-MALVACEAE, Juss.}

4983-Abutilon angulatum, Mast. (A. intermedium, Hochst.)

Fl. Trop. Afr. i. 183 ; Cat. Afr. Pl. Welw. i. 65.

Victoria Falls, Allen, 420 ; April, Flanagan, 3304 ; Rogers, 5114,

7122 ; Umvumvumvu Riv. Swyn.; Bulawayo, Rogers.

A. Cecili, N. E. Br.

Kew Bull. 1906, 99.

Inyanga, etc. 5400-6300 ft. Cecil, 196.

A. fruticosum, Guill. \& Perr.

Fl. Trop. Afr. i. 187 ; Cat. Afr. Pl. Welw. i. 67.

Bulawayo, Dec. Rand, 27.

r Rogers has this number named $T$. pentandra, Reich. 
Ä. hirsutissimum, Moench. (A. asiaticum, Don.)

Cat. Afr. Pl. Welw. i. 66.

Bulawayo, Dec. Rand, 7.

A. matopense, Gibbs.

Journ. Linn. Soc. Bot. xxxvii. 431.

Matopos, Sep. Gibbs, 98.

A. Sonneratianum, Sweet.

Chirinda, 3800 ft. fl. \& fr. May, Swyn. 504 ; Chipinga, Swyn.; Bulawayo, Rogers, 13603*.

A. zanzibaricum, Bojer.

Fl. Trop. Afr. i. 186 ; Cat. Afr. Pl. Welw. i. 66.

Mazoe, $4500 \mathrm{ft}$. April, Eyles, 354.

4985-_Wissadula hernandioides, Garcke.

Victoria Falls, 3000 ft. May, Eyles, 142.

W. rostrata, Planch.

Fl. Trop. Afr. i. 182.

Victoria Falls, July, Kolbe, 3154; April, Flanagan, 3297 ; Rogers, 5011.

4995-Malvastrum, spp.

Khami Riv. 4500 ft. Oct. Eyles, 12.

Bulawayo, Rogers, 13602.

Victoria Falls, Rogers, 7170.

4998-Sida acuta, Burm. (S. carpinifolia, L.)

Fl. Trop. Afr. i. 180 ; Cat. Afr. Pl. Welw. i. 63.

Salisbury, Rogers, 4020.

S. cordifolia, $L$.

Fl. Trop. Afr. i. 181 ; Fl. Cap. i. 168 ; Cat. Afr. Pl. Welw. i. 64.

Victoria Falls, 3000 ft. May, Eyles, 145 (forma); Allen, 72 ; Rogers, 5709; Matopos, Nov. Marloth, 3358; Rogers, 5185, 7088 ; Gwelo, Sr. Phil. 32 ; Chipetzana \& Chipinga, Swyn.; Bulawayo, Rogers, 5759.

S. longipes, E. Mey. (S. capensis, E. \& Z.)

Fl. Cap. i. 167.

Bulawayo, 4500 ft. Jan. Eyles, 3 ; Dec. Rand, 30 ; fl. \& fr. May, Rand, 292; Matopos, Sep. Gibbs, 79; Engler; Gwelo, Sr. Phil. 28; Victoria Falls, Rogers, 5627, 7168; Salisbury, Rogers, 5799; Matabeleland, Marloth.

var. canescens, Szyszyl.

Bulawayo, Rogers, 5735.

\section{S. rhombifolia, $L$.}

Fl. Trop. Afr. i. 181 ; Fl. Cap. i. 167 ; Cat. Afr. Pl. Welw. i. 64.

Bulawayo, Dec. Gardner, 91 ; Mazoe, 4500 ft. March, Eyles, 272 Victoria Falls, Rogers, 7073. 
Genus No.

\section{S. spp.}

Bulawayo, Chubb, 304, 355.

Victoria Falls, Rogers, 5153.

Salisbury, Rogers, 5768, probably sp. n.

5006-Urena lobata, $L$.

Fl. Trop. Afr. i. 189 ; Cat. Afr. Pl. Welw. i. 67 ; For. Fl. Port. E. Afr. 17.

Victoria Falls, 3000 ft. May, Eyles, 146 ; April, Flanagan, 3278 ; Rogers, 5108, 6021.

5007-Payonia clathrata, Mast.

Fl. Trop. Afr. i. 193.

North of Bulawayo, 3400 ft. Dec. Eyles, 1124; Bulawayo, Rogers, 13603*.

P. columella, Cav.

Chirinda, 3800 ft. April, Swyn. 299 ; May, Swyn. 525; Melsetter, $6000 \mathrm{ft}$. Swyn. 2049.

P. macrophylla, E. Mey.

Fl. Trop. Afr. i. 190 ; Fl. Cap. i. 169.

Mazoe, 4300 ft. Dec. Eyles, 205 ; Khami Riv. 4500 ft. Feb. Eyles, 1198; Victoria, Monro, 848; Salisbury, Rogers, 5794 ; Bulawayo, Rogers, 5761, 13603; South Rhodesia, Rand, 29.

P. Meyheri, Mast. (P.galpiniana, Schinz.)

Fl. Trop. Afr. i. 191.

Salisbury, March, Flanagan, 3021 ; Mazoe, 4500 ft. April, Eyles, 355 ; Odzani River Valley, Umtali, Teague, 98.

P. schimperiana, Hochst.

Fl. Trop. Afr. i. 192.

Chirinda, 3700-4000 ft. May, Swyn. 523.

\section{P. spp.}

Bulawayo, Chubb, 338 ; Rogers, 5762.

Matopos, Rogers, 5168.

Victoria Falls, Rogers, 5710, 7202.

South Rhodesia, Rand, 94.

5013-Hibiscus æthiopicus, $L$.

Fl. Cap. i. 174.

North Melsetter, 5000-6000 ft. Oct. Swyn. 6222; Bulawayo, Monro, 103 (var.).

H. Allenii, Sprague \& Hutch.

Kew Bull. 1907, 45.

Victoria Falls, Allen, 103.

H. articulatus, Hochst.

Fl. Trop. Afr. i. 200.

Gwelo, Jan. Gardner, 32 ; Victoria Falls, Rogers, 7240a. 
A Record of Plants Collected in Southern Rhodesia.

Genus No.

5013-H. calycinus, Willd. (H. calyphyllus, Cav.)

Fl. Trop. Afr. i. 202 ; Fl. Cap. i. 170 ; Cat. Afr. Pl. Welw. i. 72.

Bulawayo, Rogers, 5760.

H. cannabinus, $L$.

Fl. Trop. Afr. i. 204 ; Fl. Cap. i. 176 ; Cat. Afr. Pl. Welw. i. 72.

Bulawayo, fl. \& fr. May, Rand, 293 ; Victoria Falls, Rogers, 7192, 7124, 7418; Chirinda, Swyn.; Odzani River Valley, Umtali, Teague, 130.

H. diversifolius, Jacq.

Fl. Trop. Afr. i. 198; Fl. Cap. i. 171.

Victoria Falls, Rogers, 5275.

H. furcatus, Roxb.

Fl. Cap. i. 176 ; Fl. Trop. Afr. i. 201.

Victoria Falls, Rogers, 7007*.

H. gossypinus, Thunb.

Fl. Trop. Afr. i. 205 ; Fl. Cap. i. 175.

Chirinda, 3500 ft. July, Swyn. 2053.

H. Kirkii, Mast.

Fl. Trop. Afr. i. 199.

Matopos, Rogers, 5367\%.

H. micranthus, L. (H. rhodanthus, Gürke.)

Fl. Trop. Afr. i. 205 ; Cat. Afr. Pl. Welw. i. 74.

Matopos, fl. \& fr. Oct. Gibbs, 255 (forma) ; Engler; Rogers, 5649 ; Salisbury, Darling, in Herb. Bolus, 10765 (var.); Hartley, Engler; Victoria, Monro.

forma macranthus?.

Salisbury, Sep. Rand, 438, 591; Mazoe, 4300-4700 ft. Aug. Eyles, 183.

H. mutatus, N. E. Br.

Kew Bull. 1906, 99.

Matopos, Cecil, 108.

H. panduriformis, Burm.

Fl. Trop. Afr. i. 203 ; Cat. Afr. Pl. Welw. i. 72.

Mazoe, 4400-4800 ft. Jan. Eyles, 531 ; Salisbury, Rogers, 5771 ; Kolbe, 4189 ; South Rhodesia, Govt. Herb. 737.

H. pentaphyllus, F. Muell.

Fl. Trop. Afr. i. 199.

Victoria Falls, April, Flanagan, 3158; Rogers, 5584.

F.physaloides, Guill. \& Perr. (H. caesius, Garcke.)

Fl. Trop. Afr. i. 199 ; Fl. Cap. i. 172 ; Nat. Pl. 319 ; Cat. Afr. Pl. Welw. i. 69.

Victoria Falls, Allen, 71 ; Rogers, 7209 ; Ngomi, April, Flanagan, 3292 ; Matopos, Flanagan, 3008 ; Rogers, 5645. 
Fl. Cap. i. 175 ; Cat. Afr. Pl. Welw. i. 75.

Bulawayo, Jan. Rand, 25 ; Rogers, 5744; Matopos, March, Eyles, 110 ; Victoria Falls, Rogers.

H. rhodesicus, Baker $f$. Journ. Bot. 1899, 424.

Bulawayo, fl. \& fr. Dec. Rand, 28.

H. Schinzii, Gïrke.

Victoria Falls, Rogers, 5016.

H. shirensis, Sprague \& Hutch.

Chirinda, 3700-4000 ft. Swyn. 298.

H. Solandra, L'Hér.

Fl. Trop. Afr. i. 206 ; Cat. Afr. Pl. Welw. i. 74.

North of Bulawayo, May, Eyles, 81.

H. surattensis, $L$.

Fl. Trop. Afr. i. 201 ; Fl. Cap. i. 177 ; Nat. Pl. 358 ; Cat. Afr. Pl. Welw. i. 71

Victoria Falls, April, Flanagan, 3157; Chirinda, Swyn.

H. Swynnertonii, Baker $f$.

Journ. Linn. Soc. Bot. xl. 28.

Sabi Riv. 1000 ft. Swyn.

H. ternatus, Mast.

Fl. Trop. Afr. i. 206.

Bulawayo, Jan. Rand, 69; Victoria Falls, Rogers, 7004.

H. Trionum, $L$.

Fl. Trop. Afr. i. 196 ; Fl. Cap. i. 176.

Bulawayo, Dec. Rand, 6; Rogers, 5745; Gwelo, Sr. Phil. 7.

H. vitifolius, $L$.

Fl. Trop. Afr. i. 197 ; Cat. Afr. Pl. Welw. i. 68.

Victor ia Falls, Allen, 116 ; Rogers, 5050, 7095 ; Matopos, Rogers, 5658 ; South Rhodesia, Rand, 26.

\section{H. spp.}

Victoria Falls, April, Flanagan, 3232 ; Allen, 113, 133 ; Rogers, 5121.

Bulawayo, Monro, 22.

Mazoe, $4300 \mathrm{ft}$. Dec. Eyles, 225.

Victoria, Monro, 1098.

South Rhodesia, Rand, 74.

5015-Kosteletzkya Büttneri, Giurke.

Cat. Afr. Pl. Welw. i. 68.

Mazoe, $4300 \mathrm{ft}$. Dec. Eyles, 224. 
Genus No.

K. spp.

Victoria Falls, April, Flanagan, 3281, ef. K. flava, Bak. f. Rogers, 5010, 5113.

5018-Thespesia garckeana, $F$. Hoffm.

Salisbury, Engler; Umtali, Engler; Govt. Herb. 834.

\section{T. spp.}

Victoria Falls, Allen, 277 ; Rogers, 5142, 5389.

Bulawayo, 4700 ft. Feb. Eyles, 1196.

South Rhodesia, Rand, 430.

5020-Gossypium sp.

Bulawayo, Monro, 30.

Family CLXXVII.-BOMBACACEAE, K. Schum.

5023-Adansonia digitata, $L$.

Fl. Trop. Afr. i. 212 ; Cat. Afr. Pl. Welw. i. 79 ; For. Fl. Port. E. Afr. 16.

Victoria Falls, Davy; Gwanda, Noble, 39 ; Sabi Valley, Inyamadzi Riv. and Tanganda Riv. Swyn.

Family CLXXVIII.-STERCULIACEAE, Schott. \& Endl.

5047-Melhania acuminata, Mast.

Fl. Trop. Afr. i. 231.

Victoria Falls, Rogers, 7051*.

M. ferruginea, A. Rich.

Fl. Trop. Afr. i. 231 ; Cat. Afr. Pl. Welw. i. 88.

Victoria Falls, Rogers, 5611.

M. Forbesii, Planch.

Fl. Trop. Afr. i. 231 ; Cat. Afr. Pl. Welw. i. 88.

Victoria, Monro, 751.

M. linearifolia, Sond.

Fl. Cap. i. 222.

North of Bulawayo, 3400 ft. Dec. Eyles, 1127; Matabeleland, Marloth.

M. obtusa, N. E. Br.

Kew Bull. 1906, 99.

Bulawayo, Cecil, 94; Matopos, Oct. Gibbs, 283; March, Flanagan, 2979.

M. prostrata, $D C$.

Fl. Cap. i. 222.

Salisbury, Engler. 
Genus No.

forma latifolia?.

Bulawayo, Dec. Rand, 24.

M. Randii, Baker $f$.

Salisbury, fl. \& fr. Aug. Rand, 439 ; Rogers, 4058, 4007 ; May, Flanagan.

M. sp.

Mazoe, 5000 ft. Sep. Eyles, 411.

5053-Dombeya densiflora, Planch.

Fl. Cap. ii. 589.

Matopos, Davy; Odzani River Valley, Umtali, Teague, 248.

D. reticulata, K. Schum.

Chirinda, July, Swyn. 47.

D. rosea, Baker $f$.

Journ. Linn. Soc. Bot. xl. 29.

Chirinda, 3900 ft. April, Swyn. 196.

D. rotundifolia, Harv.

Fl. Cap. i. 221 ; Nat. Pl. 229.

Salisbury, July, Rand, 568 forma; Matopos, Sep. Gibbs, 24 ;

Engler; Bulawayo, Engler; Aug. Eyles, 1066 ; Chubb, 343 ;

Victoria Falls, Allen, 419; Rogers, 5004; Mazoe, 4800 ft. July, Eyles, 377 ; Govt. Herb. 923 ; Gwanda, Noble, 70 ; Inyoka, Govt. Herb. 951.

5056-Hermannia boraginiflora, Hook.

Fl. Cap. i. 201.

Victoria Falls, July, Kolbe, 3179; Gwelo, Sr. Phil. 35; Bulawayo, Oct.-Dec. Chubb, 303.

H. brachypetala, Harv.

Fl. Cap. i. 202.

Matopos, Sep. Gibbs, 7 ; Galpin, 7004.

H. depressa, N. E. Br.

Salisbury, July, Rand, 569 ; Bulawayo, May, Rand, 332 ; Monro, 107 ; Mazoe, 4300-4800 ft. Sep. Eyles, 423.

H. filipes, Harv.

Fl. Trop. Afr. i. 232 ; Fl. Cap. i. 206.

Victoria Falls, Rogers, 5732.

H. quartiniana, Rich. (Mahernia abyssinica, Hochst.)

Fl. Trop. Afr. i. 234 ; Fl. Cap. i. 216.

Matopos, fl. \& fr. Sep.-Oct. Gibbs, 77.

H. viscosa, Hiern.

Bulawayo, Monro, 59 ; Matopos, Sep. Gibbs, 5 ; Victoria Falls, April, Flanagan, 3228. 
Genus No.

var. Randii, Baker $f$.

Journ. Bot. 1901, 128.

Bulawayo, May, Rand, 295.

\section{H. spp.}

Kesi, April, Flanagan, 3283.

Bulawayo, $4500 \mathrm{ft}$. Jan. Eyles, 2.

South Rhodesia, Rand, 18.

5057-Melochia corchorifolia, $L$.

Fl. Trop. Afr. i. 236 ; Cat. Afr. Pl. Welw. i. 90.

Victoria Falls, Allen, 397.

5059-Waltheria americana, L. (W. indica, L.)

Fl. Trop. Afr. i. 235 ; Fl. Cap. i. 180 ; Cat. Afr. Pl. Welw. i. 91.

Bulawayo, Dec. Rand, 53; Matopos, Galpin, 6957; between

Plumtree and Bulawayo, April, Flanagan, 3180; Gwelo, Sr.

Phil. 40 ; Victoria Falls, Rogers, 5001 ; 7159, 7201 ; Chimanimani Mts. 7000 ft. Sep. Swyn. 2057; Chipetzana, 3000 ft. April, Swyn. 2058; Odzani River Valley, Umtali, Teague, 205.

5083-Sterculia diversicolor?.

Matopos, Govt. Herb. 902.

S. livingstoneana, Engl.

Victoria Falls, Sep. Engler, 2936.

S. spp.

Between Malindi and Victoria Falls, Galpin, 7056a.

Victoria, Monro, 885.

\section{Series PARIETALES. \\ Family CLXXXII.-OCHNACEAE, $D C$.}

5112_-Ochna Antunesii, Engl. \& Gilg.

Victoria Falls, Sep. Gibbs, 305; Allen, 50 ; Rogers, 5301, 5302, 7450, 7459 .

0. chirindica, Baker $f$.

Journ. Linn. Soc. Bot. xl. 37.

Chirinda Forest, 3700 ft. Oct. Swyn. 106, leaf in May.

0. leptoclada, Oliv.

Fl. Trop. Afr. i. 318.

Chikore Hills, 3500 ft. Nov. Swyn. 1356; Mt. Pene, 7000 ft. Oct. Swyn. 6172.

0. pulchra, Hook $f$.

Fl. Trop. Afr. i. 317 ; Fl. Cap. i. 449 ; Cat. Afr. Pl. Welw. i. 121. Matopos, Davy; North of Bulawayo, Engler; Victoria Falls, Rogers, 7450. 
0. schweinfurthiana, $F$. Hoffm.

Matopos, fl. \& fr. Oct. Gibbs, 218.

0. spp.

Victoria Falls, Sep. Galpin, 7050 ; Allen, 172 cf. O. Antunesii, Engl. \& Gilg.

Salisbury, Rand, 1351 cf. O. humilis, Engl.

Bulawayo, Eyles, 1184.

Chipete Forest, 3800 ft. Swyn. cf. O. chirindica, Bak. f.

Charter, Govt. Herb. 3021.

Mazoe, 4600 ft. Jan. Eyles, 506.

5114-Brackenridgea zanguebarica, Oliv. (Pleuroridgea zanguebarica, Van Tieghem.)

Chirinda, 3600 ft. fr. Dec. Swyn. 189 ; Chikore, Swyn.

Family CLXXXVII.-GUTTIFERAE, Juss.

5168-Hypericum æthiopicum, Thunb.

Fl. Cap. i. 117.

Chirinda, 3800 ft. April, Swyn. 374 ; May, Swyn. 508; between Umtali and Imyanga Mts. Cecil.

H. Lalandii, Choisy.

Fl. Trop. Afr. i. 155 ; Fl. Cap. i. 118 ; Cat. Afr. Pl. Welw. i. 56.

Matopos, Gibbs ; Rogers, 5175, 5364 ; Victoria, Monro, 1021 ; Mt. Pene, 6000 ft. Oct. Swyn. 6173.

H. lanceolatum, Lam.

Fl. Trop. Afr. i. 156; For. Fl. Port. E. Afr. 14.

Melsetter, 6000 ft. Sep. Swyn. 681, 681a; Mt. Pene, 7000 ft. Oct. Swyn. 6091 ; between Melsetter anł Umvumvumvu Riv. Swyn.

5171-Vismia corymbosa, A. Chev.

Victoria Falls, Rogers, 7245.

5172-Psorospermum febrifugum, Spach.

Fl. Trop. Afr. i. 158 ; Cat. Afr. Pl. Welw. i. 57 ; For. Fl. Port. E. Afr. 14.

Victoria Falls, Rogers, 7259 ; Chirinda, 3800 ft. Oct. Swyn. 195.

P. sp.

Salisbury, Rand, 1367.

5173-Haronga madagascariensis, Choisy. (Harungana paniculata, Pers.)

Fl. Trop. Afr. i. 160 ; Cat. Afr. Pl. Welw. i. 58 ; For. Fl. Port. E. Afr. 14.

Chirinda, 3800 ft. fl. \& fr. Feb. Swyn. 161. 
G199-Garcinia Livingstonei, T. And.

Fl. Tr p. Afr. i. 165 ; For. Fl. Port. E. Afr. 15.

Victoria Falls, Galpin, 7024 ; Davy; Sep. Gibbs, 114; Allen, 190 ; Rogers, 5316, 7463 ; Engler.

G. sp.

Chimanimani Mts. Swyn. 574.

Family CLXXXVIII.-DIPTEROCARPACEAE, Heim.

5228-Monotes africanus, A.DC. var. denudans, Hiern. (Vatica africana, Welw. var. laxa, Oliv.)

Fl. Trop. Afr. i. 173; Cat. Afr. Pl. Welw. i. 61.

Mazoe, 4300 ft. Ja’. Eyles, 242 ; Salisbury, Rand, 1398.

M. glaber, Sprague. (Vatica africana, Welw. var. glabra, Oliv.)

Kew Bull. 1909, 305 ; Fl. Trop. Afr. i. 173.

Hunyani Valley, Allen, 734 ; Salisbury, Rand, 557.

M. hypoleucus, Gilg. (Vatica africana, Welw. var. hypoleuca, Oliv.)

Fl. Trop. Afr. i. 173 ; Cat. Afr. Pl. Welw. i. 62.

Mazoe, $5000 \mathrm{ft}$. Dec. Eyles, 211 ; between Inyamadzi and Buzi, 3400 ft. Dec. Swyn. 157 ; Upper Buzi, 3000 ft. fr. Ost. Swyn. 1314 ; Chirinda, Swyn.

M. sp.

Victoria, Monro, 870, ef. M. Engleri, Gilg.

Family CLXXXIX.-ELATINACEAE, Lindl.

5230-Bergia decumbens, Planch.

Fl. Trop. Afr. i. 153; Fl. Cap. i. 116.

Bulawayo, Rand, 333 and 337 p.p.; Oct. Eyles, 1092 ; Monro, 69, 236; Rogers, 5480; Matopos, Sep--Oct. Gibbs, 30; Engler.

Family CXCVIII.-VIOLACEAE, $D C$.

5262-Rinorea convallarioides (Baker f.), Torre \& Harms. (Alsodeia convallarioides, Baker f.)

Journ. Linn. Soc. Bot. xl. 21.

Chirinda Forest, Sep. Swyn. 2119; fr. Oct. 2119a; also Swyn. $1110 \mathrm{fr}$. only taken from same shrub as flowers. 
R. gazensis (Baker f.), Torre \& Harms. (Alsodeia gazensis, Baker f.) Journ. Linn. Soc. Bot. xl. 22.

Chirinda Forest, 3700-4000 ft. Oct. Swyn. 132 ; fr. Dec. Swyn. 6500 .

5271-Hybanthus enneaspermum (Vent.), Torre \& Harms. (Ionidium enneaspermum, Vent.)

Fl. Trop. Afr. i. 105.

Bulawayo, Rand, 58 ; Monro, 95.

H. sp.

Bulawayo, $4500 \mathrm{ft}$. Nov. Eyles, 1214.

5274-Viola abyssinica, Steud.

Fl. Trop. Afr. i. 105.

Mt. Pene, 7000 ft. Oct. Swyn. 6203.

\section{Family CXCIX.-FLACOURTIACEAE, Dumort.}

5275-Rawsonia lucida, Harv. \& Sond.

Fl. Cap. i. 67 ; Nat. Pl. 575 ; For. Fl. Port. E. Afr. 12 ; For. Fl. Cape, 128.

Chirinda Forest, 3800 ft. Oct. Swyn. 658.

5284-Oncoba spinosa, Forsk.

Fl. Trop. Afr. i. 115 ; Fl. Cap. ii. 583 : For. Fl. Port. E. Afr. 12 ; Cat. Afr. Pl. Welw. i. 38.

Victoria Falls, Sep. Gibbs, 304.

0. sp.

Victoria Falls, Allen, 110 ef. O. spinosa, Forsk.

5296-Kiggelaria africana, $L$.

Fl. Cap. i. 71 ; For. Fl. Cape, 128.

Chimanimani Mts. 7000 ft. Swyn. 2039 ; Mt. Pene Forest, 6500 7000 ft. bud Sep. Swyn. 2040.

5299-Paropsia reticulata, Engl.

Victoria Falls, Allen, 135 ; April, Flanagan, 3205 ; July, Kolbe, 3133 ; Rogers, 5629, 7460, 7161.

5326-Neumannia theæformis (Benn.), Torre \& Harms. (Aphloia theceformis, Benn.)

Chimanimani Mts. 7000 ft. Sep. Swyn. 634 ; Mt. Pene, 60006500 ft. Sep. Swyn. $634 a$; fr. Oct. Swyn. 6168.

5327-Flacourtia hirtiuscula, Oliv.

Fl. Trop. Afr. i. 121.

Victoria, Monro, 638.

F. Ramontchi, L'Hér.

Fl. Trop. Afr. i. 120 ; For. Fl. Port. E. Afr. 13.

Matopos, Engler. 
Genus No.

\section{F. spp.}

Victoria Falls, Allen, 192.

Victoria, Monro, 527.

Matopos, Rogers, 5895, 5696.

South Rhodesia, Rand, 605.

5328-Doryalis (Doyyalis) caffra, Sim. (Aberia caffra, Hook. f.)

Fl. Cap. ii. 584 ; For. Fl. Port. E. Afr. 13 ; For. Fl. Cape, 129 ; Harv. Gen. S. A. Pl. 16.

Gwanda, Noble, 45 ; Victoria, Monro, 472 ; Lomagundi, Govt. Herb. 977.

D. macrocalyx, Warb. (Aberia ? macrocalyx, Oliv.)

Fl. Trop. Afr. i. 122 ; Cat. Afr. Pl. Welw. i. 40.

Chirinda Forest, 3800-4000 ft. fr. Oct. Swyn. 68.

D. Zeyheri (Sond.), Torre \& Harms. (Aberia Zeyheri, Sond.)

Fl. Cap. i. 70.

Victoria, Monro, 545, 763 ; Victoria Falls, Rogers, 13057*.

D. sp.

Victoria, Monro, 833.

Family CCI.-TURNERACEAE, $H . B . \& K$.

\section{5-Wormskioldia lobata, Urb.}

Cat. Afr. Pl. Welw. i. 381.

Bulawayo, Dec. Rand, 13 ; Victoria, Monro, 686, 817, 897 ; Victoria Falls, Rogers, 5631* 7013*.

W. longepedunculata, Mast.

Fl. Trop. Afr. ii. 502.

Matabeleland, Oates; Bulawayo, Dec. Rand, 20 (forma); Nov. Eyles, 24; Salisbury, fl. and fr. Sep. Rand, 613 ; May, Flanagan, 3254 ; Matopos, Sep. Gibbs, 48 ; Victoria Falls, Gibbs; Allen, 95 ; North of Hartley, Engler ; Rusapi, Engler ; Mazoe, 4300-4800 ft. Nov. Eyles, 453 ; Victoria, Monro, 687 ; Gwelo, Sr. Phil. 24.

\section{W. petersiana?.}

South Rhodesia, Rand.

W. tanacetifolia, Klotzsch. (? W. heterophylla, Schum.)

See note Fl. Trop. Afr. ii. 503.

Victoria, Monro, 1053.

\section{W. sp.}

Victoria Falls, Allen, 142.

5356-Streptopetalum serratum, Hochst.

Bulawayo, Jan. Rand, 68; Victoria Falls, Rogers, 7253. 
FAMily CCIII.--PASSIFLORACEAE, Lindl.

Genus No.

5369-Tryphostemma apetalum, Baker $f$. var. serratum, Baker $f$.

Journ. Bot. 1899, 437.

Salisbury, fl. and fr. Dec. Marshall; April, Flanagan, 3150 ;

Rand, 1347 ; Rogers, 4022 ; Gwibi Flats, 5000 ft. Sep. Eyles, 412 ; Rusapi, Engler; Umtali, Engler; Mazoe, 4300 ft. Nov. Eyles, 461.

\title{
T. Mastersii ?.
}

South Rhodesia, Rand.

T. parvifolium, Baker $f$.

Journ. Linn. Soc. Bot. xl. 73.

Between Lusitu and Melsetter, $6000 \mathrm{ft}$. Sep. Swyn. 1415.

T. pedatum, Baker $f$. Journ. Bot. 1899, 436.

Shasi Riv. Jan. Rand, 67.

T. spp.

Salisbury, Rand, 470.

South Rhodesia, Flanagan, 3159.

5370-Adenia senensis, Engl.

Salisbury, Rand, 1345, 1346; Bulawayo, Monro, 68.

\author{
Series OPUNTIALES. \\ Family CCX.-CACTACEAE, Lindl.
}

5416-Rhipsalis Cassytha, Gaertn. (Hariota parasitica, O. Kuntze.)

Fl. Trop. Afr. ii. 581 ; Fl. Cap. ii. 480 ; Cat. Afr. Pl. Welw. i. 407 ;

Nat. Pl. 394.

Chirinda Forest, 3700-4000 ft. Nov. Swyn. 1086; Chipete, Swyn.

\section{SERIES MYRTIFLORAE.}

Family CCXIII.-OLINIACEAE, C. Presl.

5428-Olina yanguerioides, Baker $f$.

Journ. Linn. Soc. Bot. xl. 72.

Umswirizwi Riv. 3500 ft. Dec. Swyn. 158.

EAmily CCXIV.-THYMELAEACEAE, Reichb.

5434-Peddiea Dregei, Meisn.

Chipete Forest, Jan. Swyn. 123 ; Mt. Pene, 6500 ft. Swyn. 123a;

Chirinda Forest, Swyn. 
Genus No.

Bulawayo, Chubb, 57.

G. Buchananii, Gilg.

Fl. Trop. Afr. VI. i. 219.

Nyahodi Riv. 5000 ft. Sep.-Oct. Swyn. 1834, 6136.

G. chrysantha, Gilg. (Arthrosolen chrysantha, Solms-Laub.)

Fl. Trop. Afr. VI. i. 234.

Between Umtali and Salisbury, Cecil, 55.

G. kraussiana, Meisn. (Lasiosiphon Kraussii, Meisn.)

Fl. Trop. Afr. VI. i. 231 ; Nat. Pl. 256 ; For. Fl. Cape, 302.

Matopos, Oct. Gibbs, 229 ; Engler; Mazoe, 4300-4800 ft. Aug.

Eyles, 396 ; Salisbury, Rogers, 4015, 4016 ; North of Hartley,

Engler ; Mt. Pene, 6500-7000 ft. Sept. Swyn. 1787 ; Bulawayo, Chubb, 307 ; Rogers, 5920.

G. microcephala, Meisn .

Fl. Trop. Afr. VI. i. 225.

North of Hartley, Engler; Salisbury, Rand, 1373; Victoria, Monro, 888.

G. microphylla, Meisn.

Matopos, $4800 \mathrm{ft}$. Nov. Eyles, 1186.

G. phyllodinea, S. Moore.

Journ. Linn. Soc. Bot. xl. 186.

Melsetter, $6000 \mathrm{ft}$. April, Swyn. 1833.

G. roridus (S. Moore), Torre \& Harms. (Lasiosiphon roridus, S. Moore.)

Journ. Linn. Soc. Bot. xl. 187.

Melsetter, $6000 \mathrm{ft}$. Oct. Swyn. 6134 .

G. spp.

Salisbury, Rand, 528, 529, 530 ; March, Flanagan, 3024 ; Darling, in Herb. Bolus 10787.

Bulawayo, Chubb, 385, 366.

South Rhodesia, Rand, 204, 205, 390, 643, 646.

5442-Synaptolepis longiflora, Gilg.

Fl. Trop. Afr. VI. i. 246.

Chirinda, 3500 ft. Oct. Swyn. 188.

Family CCXVI.-LYTHRACEAE, Lindl.

5473-Rotala cataractae Koehne.

Victoria Falls, Sep. Engler, 2990.

R. filiformis, Hiern.

Fl. Trop. Afr. ii. 468 ; Cat. Afr. Pl. Welw. i. 372.

Victoria Falls, Kirk. 
R. heteropetala, Koehne, var. Engleri, Koehne.

Victoria Falls, Sep. Engler, 2983.

R. longistyla, Gibbs.

Journ. Linn. Soc. Bot. xxxvii. 445.

Victoria Falls, Sep. Gibbs, 170.

\section{R. spp.}

Victoria Falls, 3000 ft. May, Eyles, 124 ; Rogers, 7416.

Victoria, Monro, 1072.

5474-Ammannia baccifera, $L$.

Fl. Trop. Afr. ii. 478 ; Cat. Afr. Pl. Welw. i. 374.

Ngomi, April, Flanagan, 3291; Victoria Falls, Rogers, 7096*.

\section{A. senegalensis, Lam.}

Fl. Trop. Afr. ii. 477 ; Cat. Afr. Pl. Welw. i. 373.

Bulawayo, May, Rand, 337, 574; Victoria, Monro, 1016, 1093.

\section{A. sp.}

Victoria, Monro, 1050.

5476-Lythrum sagittifolium, Sond.

Fl. Cap. ii. 516.

Bulawayo, 4500 ft. Oct. Eyles, 1070.

5486-Nesæa erecta, G. \& P.

Fl. Trop. Afr. ii. 474.

Victoria Falls, Rogers, 7150*.

N. floribunda, Sond.

Fl. Trop. Afr. ii. 474 ; Fl. Cap. ii. 517 ; Cat. Afr. Pl. Welw. i. 376.

Victoria Falls, Galpin, 7032 ; Sep. Gibbs, 164 ; Kirk ; Allen, 38 ; Rogers, 5237 ; Matopos, Gibbs ; Umtali, Engler; Gwelo, Sr. Phil. 29 ; Victoria, Monro, 815 \& 348 ?

N. heptamera, Hiern.

Fl. Trop. Afr. ii. 472.

Marandellas, Rogers, 4039.

N. mucronata, Koehne.

Bulawayo, Chubb, 350, 324.

N. passerinoides, Koehne.

Cat. Afr. Pl. Welw. i. 377.

Salisbury, April, Flanagan, 3260.

N. radicans, Guill. \& Perr.

Fl. Trop. Afr. ii. 474 ; Cat. Afr. Pl. Welw. i. 376.

Victoria Falls, 3000 ft. May, Eyles, 122 (forma); Engler ; Rogers, 5123.

N. rigidula, Koehne.

Khami Riv. 4500 ft. Oct. Eyles, 1084.

N. sagittifolia, Sond.

Bulawayo, May, Rand, 330. 
Genus No

N. Stuhlmannii, Koehne.

Marandellas, Engler.

N. triflora, H. B. Kunth.

Salisbury, Sep. Rand, 588.

N. spp.

Gwai, Jan. Allen, 246.

Victoria, Monro, 1049.

Bulawayo, Chubb, 365.

Salisbury, Rand, 1390 cf. N. linifolia, Hiern.

Family CCXX.-RHIZOPHORACEAE, Lindl.

5528-Weihea Gerrardi, Schinz.

Mt. Pene Forest, 6500-7000 ft. Sep. Swyn. 1325.

FAmily CCXXI.-COMBRETACEAE, $R . B r$.

5538-Combretum abbreviatum, Engl.

Chirinda Forest, Oct. Swyn. 95.

C. apiculatum, Sond.

Fl. Trop. Afr. ii. 429 ; Fl. Cap. ii. 510 ; For. Fl. Port. E. Afr. 63.

Bulawayo, Jan. Rand, 33 ; Nyamandhlovu, fr. April, Flanagan, 3195 ; Sabi Riv. 1000 ft. Nov. Swyn. 700 ; Victoria Falls, Rogers, 5569, 552う̃ ; Wankie, Rogers, 5992.

var. parvifolium, Baker $f$.

Journ. Bot. 1905, 46.

Bulawayo, $4500 \mathrm{ft}$. Oct. Eyles, 1094.

C. arbuscula, Engl.

Bulawayo, Monro, 6 \& 84; Chirinda, 3700 ft. Oct. Swyn. 43; Inyamadzi Valley, fr. April, Swyn. 43a.

C. atelanthum, Diels.

Salisbury, Sep. Engler, 3098.

C. Bragae, Engl.

Umtali, Engler.

C. cataractarum, Diels.

Victoria Falls, Sep. Engler, 2925 ; Allen, 46; Sep. Gibbs, 127 ;

Galpin, 7048 ; Rogers, 5289 ; Wankie, Rogers, 5994.

C. cognatum, Diels.

North of Bulawayo, Sep. Engler, 2885.

C. erythrophyllum, Sond.

Fl. Cap. ii. 509 ; For. Fl. Cape, 222.

Bulawayo, Monro, 89 ; Nyahodi Riv. 5500 ft. Sep. Swyn. 701. 
Fl. Cap. ii. 509.

Bulawayo, Rand, 585 ; Monro, 87, 88 ; Chubb, 50.

C. Gueinzii, Sond.

Fl. Cap. ii. 509.

Khami, Nov. Marloth, 3389 ; Matopos, Engler; Victoria Falls, Rogers, 5571.

C. hereroense, Schinz.

Bulawayo, Engler.

C. holosericeum, Sond.

Fl. Trop. Afr. ii. 430; Fl. Cap. ii. 510 ; Cat. Afr. Pl. Welw. i. 350 ; For. Fl. Port. E. Afr. 63.

Matabeleland, Oates; Marloth; Mashonaland, Mundy.

C. imberbe, Wawra.

South of Zambesi, Engler.

C. Junodii, Dümmer.

Victoria, Monro, 481, 508.

C. Lindenbergianum, Engl. \& Diels.

Victoria, Monro, 499; Mashonaland, Mundy.

C. microphyllum, Klotzsch.

Fl. Trop. Afr. ii. 427 ; For. Fl. Port. E. Afr. 63.

Victoria, Monro, 467 ; Sabi Riv. 1000 ft. Nov. Swyn. 2083.

C. mossambicense, Engl.

Sabi Riv. 1000 ft. Nov. Swyn. 590, 591.

C. oblongum, F. Hoffm.

Victoria Falls, Rogers, 5792 ?

C. patelliforme, Engl. \& Diels.

Victoria Falls, Rogers, 5589.

C. platypetalum, Welw. (C. Oatesii, Rolfe.)

Fl. Trop. Afr. ii. 433 ; Cat. Afr. Pl. Welw. i. 353.

Matabeleland, Oates; Bulawayo, Sep. Rand, 322 ; Nov. Eyles, 1109 ; Salisbury, Sep. Rand, 466; Rogers, 4060 ; Darling, in Herb. Bolus, 10775 ; Khami, Nov. Marloth, 3392; Matopos, S'ep. Gibbs; Mazoe, 4300 ft. Sep. Eyles, 432; Gwibi Flats, $5000 \mathrm{ft}$. Oct. Eyles, 441 ; North of Hartley, Engler; Victoria, Monro, 451, 567, 504, 544 ; Marandellas, Rogers, 4037.

C. primigenum, Marloth \& Engl.

Victoria Falls, April, Flanagan, 3227; Rogers, 5702, 5708; Victoria, Monro, 869, 646.

C. rhodesicum, Baker $f$.

Journ. Bot. 1899, 435.

Bulawayo, fl. \& fr. Sep. Rand, 582, 583 ; Rogers, 5924, 5502 ; Matopos, fl. \& fr. Oct. Gibbs, 267 ; Rogers, 5635 ; Zambesi 
Genus No.

banks, Sep. Galpin, 7013 ; Umtali-Melsetter road, 2000-3000

ft. Oct. Swyn. 6600 ; Victoria Falls, Rogers, 5534, 5353.

C. salicifolium. E. Mey.

Fl. Cap. ii. 511 ; For. Fl. Port. E. Afr. 64 ; For. Fl. Cape, 222.

Bulawayo, Monro, 80 ; Victoria, Monro, 208 ; Gwanda, Noble, 50.

C. tetraphyllum, Diels.

South of Zambesi, Sep. Engler, 2906.

C. ulugurense, Engl.

Victoria, Monro, 466.

C. Zeyheri, Sond.

Fl. Cap. ii. 511 ; For. Fl. Port. E. Afr. 63 ; Cat. Afr. Pl. Welw. i. 352 .

Matopos, Oct. Gibbs, 221 ; Mazoe, 4300 ft. Sep. Eyles, 434 ?; Umvumvumvu Riv. 2000 ft. April, Swyn. 2081; Victoria, Monro, 509, 564 ; Victoria Falls, Rogers, 5700; Bulawayo, Monro, 368, 346; Sebakwe, Rogers, 8591; Wankie, Rogers, 5990 ; between Bulawayo \& Victoria Falls, Galpin; Queque, Rogers, 5451*.

\section{C. spp.}

Bulawayo, Chubb, 339, 372.

Salisbury, Rand, 467.

Victoria, Monro, 302, 475, 535, 650, 698, 699.

Victoria Falls, Gibbs, 127 ; Rogers, 5273, 5305, 5570.

Matopos, Rogers, 5344 ; Govt. Herb. 884.

Gockwe Hills, Govt. Herb. 952, 946.

Lomagundi, Govt. Herb. 963, 969.

Mazoe, Govt. Herb. 2084.

South Rhodesia, Rand, 32, 132, 323, 324, 426, 429, 579, 580, 584, 602.

5539-Pteleopsis myrtifolia, Engl. \& Diels.

Upper Inyamadzi, Dec. Swyn. 48 ; Inyamadzi Valley, $3300 \mathrm{ft.}$ fr. April, Swyn. 2084; Chirinda, Swyn.; Chikore, Swyn.

5541-Quisqualis indica, $L$.

Fl. Trop. Afr. ii. 435.

Chirinda, cultivated, Swyn.

5544-Terminalia Brownii, Fresen.

Fl. Trop. Afr. ii. 415 ; For. Fl. Port. E. Afr. 64.

Bulawayo, Monro, 48.

T. prunioides, Laws.

Fl. Trop. Afr. ii. 415 ; For. Fl. Port. E. Afr. 64.

Victoria Falls, Allen, 122. 
T. Randii, Baker $f$.

Journ. Bot. 1899, 435.

Bulawayo, fl. \& fr. May, Rand, 325 ; Chubb, $9 a$; Rogers, 5452 ; Nov. Eyles, 1210.

T. sericea, Burch.

Fl. Trop. Afr. ii. 416; Fl. Cap. ii. 508; Cat. Afr. Pl. Welw. i. 338 ; For. Fl. Port. E. Afr. 64 ; Harv. Gen. S.A. Pl. 110. Bulawayo, 4500 ft. Eyles, 1221; Engler; Monro, 105; from Bulawayo to Victoria Falls, Galpin, 6955; Matopos, Davy; Engler; Victoria Falls, Davy ; Allen, 425; Umtali, Engler; Gwanda, Noble, 35 ; Victoria, Monro, 668 var.; Fort Gibbs, Rand, 600, 601; Gwelo, Rand, 5; Chirinda, Swyn.; Chikore Hills, Swyn.; Nyahodi Riv. Swyn.

var. angolensis, Hiern.

Cat. Afr. Pl. Welw. i. 338.

Bulawayo, May, Rand, 416.

T. silozensis, Gibbs.

Journ. Linn. Soc. Bot. xxxvii. 444.

Matopos, Silozi Hill, fl. \& fr. Oct. Gibbs, 277.

T. spinosa, Engl.

South of Zambesi, Engler.

T. Stuhlmannii, Engl.

Victoria Falls, Allen, 123 ; Rogers, 5526.

T. torulosa, F. Hoffm.

Victoria Falls, Rogers, 5568 ; Victoria, Monro, 880.

T. trichopoda, Diels.

Matopos, Sep. Engler, 2847 ; Umtali, Engler, 3142.

T. velutina, Rolfe.

Buiawayo, Chubb, 105.

T. spp.

Victoria Falls, Allen, 63 cf. T. spinosa, Engl. ; Allen, 163.

Between Plumtree and Bulawayo, fr. April, Flanagan, 3209.

\section{Family CCXXII.-MYRTACEAE, Pers.}

5578-Eugenia angolensis, Engl.

Chirinda, 3800 ft. Aug. Swyn. 242.

E. chirindensis, Baker $f$.

Journ. Linn. Soc. Bot. xl. 70.

Chirinda Forest, 3700-4000 ft. Dec. Swyn. 128; Oct. Swyn. 443 ; Chipete Forest, Oct. Swyn. 1343. 
Genus No.

E. cordata, Laws. (Syzygium cordatum, Hochst.)

Fl. Trop. Afr. ii. 438; Fl. Cap. ii. 521 ; For. Fl. Port. E. Afr. 67 ; For. Fl. Cape, 226 ; Cat. Afr. Pl. Welw. i. 360.

Victoria Falls, Allen, 53; Galpin; Engler; Umtali, Engler; Mazoe, Govt. Herb. 925 ; North of Sengwe Riv. Govt. Herb. 954 ; Chirinda, 3800 ft. Oct. Swyn. 25; Haroni Riv. 3500 ft. Nov. Swyn. 1309; Melsetter Dist. Swyn.

E. owariensis, P. Beauv. (Syzygium owariense, Benth.; Syzygium guineense, DC.)

Fl. Trop. Afr. ii. 438; For. Fl. Port. E. Afr. 67 ; Cat. Afr. Pl. Welw. i. 359.

Salisbury, Aug. Rand, 468; Sep. Rand, 586 (forma); Engler ; Mazoe, 4800 ft. Sep. Eyles, 403; Victoria Falls, Engler; Galpin, 7022 ; Allen, 69 ; Rogers, 6027; Umtali, Engler ; Victoria, Monro, 480 ; Chirinda, 3500 ft. June, Swyn. 23 ; Chimanimani Mts. 7000 ft. Sep. Swyn. 642; Mt. Pene, 6500-7000 ft. Sep. Swyn. 638; Sabi Riv. 1000 ft. Nov. Swyn. 1311; Chipete Forest, Swyn.

forma latifolia, Engl. \& Gilg.

Chirinda, 3500 ft. Oct. Swyn. 24 ; Chikore Hills, Nov. Swyn. 1304.

\section{E. spp.}

Victoria Falls, Allen, 56 ef. E. cordata, Laws.

Salisbury, Rand, 1366.

Victoria Monro, 547, 759, 764.

Mazoe, Govt. Herb. 996, 927.

5583-Syzygium benguelense (Welw.), Engl. (Eugenia benguellensis, Welw.)

Cat. Afr. Pl. Welw. i. 360.

North of Hartley, Engler.

S. huillense (Hiern), Engl. (Eugenia guineensis, var. huillensis, Hiern.)

Cat. Afr. Pl. Welw. i. 359.

North of Hartley, Engler; Salisbury, Engler; Marandellas, Engler.

S. intermedium, Engl.

Victoria Falls, Engler.

Family CCXXiII.-MeLASTOMATACEAE, $R$. $B r$.

5651-Antherotoma Naudini, Hook. f. (Osbeckia antherotoma, Naud.)

Fl. Trop. Afr. ii. 444 ; Cat. Afr. Pl. Welw. i. 363.

Chirinda, 3800 it. fr. July, Swyn. 516. 
Genus No.

5658-Osbeckia Swynnertonii, Baker $f$.

Journ. Linn. Soc. Bot. xl. 71.

Chimanimani Mts. 7000 ft. Sep. Swyn. 2085.

5659-Dissotis debilis, Triana.

Cat. Afr. Pl. Welw. i. 366.

Matopos, 4500 ft. Jan. Eyles, 153.

D. incana, Triana.

Mazoe, 4300 ft. Jan. Eyles, 509 ; Salisbury, Rogers, 4070 ;

Chirinda, 3800 ft. Dec. Swyn. 296; Odzani River Valley, Umtali, Teague, 101.

D. phæotricha, Hook.f. (Osbeckia pheotricha, Hochst.)

Fl. Trop. Afr. ii. 451 ; Fl. Cap. ii. 519.

Salisbury, Dec. Rand, 76; Matopos, 4500-5000 ft. March, Eyles, 1017 ; Victoria, Monro, 648, 815.

D. princeps, Triana.

Chirinda, 3800 ft. May, Swyn. 526; Odzani River Valley, Umtali, Teague, 227.

D. segregata, Hook. $f$.

Fl. Trop. Afr. ii. 448.

Victoria Falls, Allen, 111; Rogers, 5585, 7270.

D. spp.

Victoria Falls, April, Flanagan, 3165 cf. D. segregata.

Victoria, Monro, 348.

Family CCXXIV.-OENOTHERACEAE, Lindl.

5791-Jussieua repens, $L$. (J. diffusa, Forsk.)

Fl. Trop. Afr. ii. 488; Cat. Afr. Pl. Welw. i. 379.

Victoria Falls, Allen, 73; April, Flanagan, 3282; North of Bulawayo, Engler.

J. suffruticosa, L. (J. angustifolia, Lam. ; J.villosa, Lam.)

Fl. Trop. Afr. ii. 489; Fl. Cap. ii. 504; Cat. Afr. Pl. Welw. i. 380.

Victoria Falls, Allen, 32; May, Eyles, 120; Engler; April, Flanagan, 3233 ; Rogers, 5283; Matopos, Rogers, 5691 ; Victoria, Monro, 923.

\section{J. sp.}

Victoria, Monro, 872 cf. J. linifolia, Vahl.

5793-Ludwigia jussiæoides, Harv. (non Lam.).

Harv. Gen. S.A. Pl. 117.

Victoria Falls, $3000 \mathrm{ft}$. May, Eyles, 103.

L. pulvinaris, Gilg.

Victoria Falls, Rogers, 5286. 
Genus No.

\section{L. sp.}

Victoria Falls, April, Flanagan, 3300 cf. L. parviflora, Benth 5795-Epilobium neriophyllum, Hausskn.

Odzani River Valley, Umtali, Teague, 323.

E. hirsutum, $L$.

Fl. Trop. Afr. ii. 487 ; Fl. Cap. ii. 506.

Mazoe, 4500 ft. Feb. Eyles, 524.

E. sp.

Matopos, Rogers, 5257.

\section{SeRIES UMBBLLIFLORAE.}

FAmily CCXXVII.-ARALIACEAE, Vent.

5872-Cussonia natalensis, Sond.

Fl. Cap. ii. 568.

Matopos, fl. \& fr. Sep. Gibbs, 107 ; Engler.

C. spicata, Thunb.

Fl. Trop. Afr. iii. 32 ; Fl. Cap. ii. 568 ; For. Fl. Port. E. Afr. 70 ; For. Fl. Cape, 229.

Umtali, Engler; Chirinda, 3800 ft. Swyn. 159; Chimanimani Mts. 7000 ft. Swyn. 2092 ; Mazoe, Govt. Herb. 2087.

C. umbellifera, Sond.

Fl. Cap. ii. 570 ; For. Fl. Cape, 230.

Chimanimani Forest, fr. Sep. Swyn. 653a.

C. sp.

Victoria, Monro, 718.

Family CCXXVIII.-UMBELLIFERAE, Morison.

5893-Hydrocotyle asiatica, $L$.

Fl. Trop. Afr. iii. 6; Fl. Cap. ii. 527 ; Cat. Afr. Pl. Welw. i. 423.

Victoria Falls, Engler.

H. moschata, Forst.

Fl. Trop. Afr. iii. 5.

Chirinda Forest, 3800 ft. fr. April, Swyn. 347.

H. sp.

Victoria Falls, Rogers, 5324 ef. H. verticillata, Thunb. \& $H$. Bonariensis, Lam.

5918-Sanicula europæa, $L$.

Fl. Trop. Afr. iii. 8; Fl. Cap. ii. 533.

Chirinda Forest, 3700-4000 ft. April, Swyn. 346. 
Genus No.

5922-Älepidea propinqua, Dümmer.

Trans. R. S. S. Afr. iii. 9 ; Fl. Trop. Afr. iii. 7, as A. amatymbica, E. \& Z.

Chimanimani Mts. 4500 ft. Johnson, 184 ; Swyn. 6208; Chirinda, 3500 ft. Swyn. 233; Odzani River Valley, Umtali, Teague, 212.

A. Swynnertonii, Dümmer.

Trans. R. S. S. Afr. iii. 15.

Chimanimani Mts. 7000 ft. Swyn. 6208a.

5990-Lichtensteinia sp.

Bulawayo, $4500 \mathrm{ft}$. Sep. Eyles, 1251.

5992-Heteromorpha arborescens, Cham. \& Schlecht.

Fl. Trop. Afr. iii. 10 ; Fl. Cap. ii. 542 ; For. Fl. Port. E. Afr. 69 ; For. Fl. Cape, 229 ; Harv. Gen. S.A. Pl. 140.

Mazoe, 4400 ft. March, Eyles, 279 ; Matopos, Rogers; Victoria, Monro, 778, 895 ; Chirinda, 4000 ft. fl. \& fr. April, Swyn. 182 ; Lusitu Riv. 4000 ft. Swyn. 2091.

H. spp.

Victoria, Monro, 320.

South Rhodesia, Rand, 17, 329.

6020-Carum copticum, Benth. \& Hook.

Fl. Trop. Afr. iii. 12.

Salisbury, Govt. Herb. 752.

6038-Sium sp.

Mazoe, 4300 ft. Dec. Eyles, 228.

6054-Diplolophium zambesianum, Hiern.

Fl. Trop. Afr. iii. 18.

Salisbury, Dec. Rand, 71; July, Rand, 473 ; May, Flanagan, 3247; Mazoe, 4300-4600 ft. March, Eyles, 269 ?; Bulawayo, Rogers, $13106 *$.

6055-Physotrichia Swynnertonii, Baker $f$.

Journ. Linn. Soc. Bot. xl. 76.

Melsetter, $6000 \mathrm{ft}$. fl. and young fr. Sep. Swyn. 649.

6116-Peucedanum araliaceum, Benth. \& Hook.

Fl. Trop. Afr. iii. 21.

South of Zambesi, Engler; Rusapi, Engler ; Umtali, Engler.

P. benguellensis (Welw.), Torre \& Harms. (Lefeburia benguellensis, Welw.)

Cat. Afr. Pl. Welw. i. 430 (Lefeburia).

Chirinda, 3800 ft. fr. May, Swyn, 395.

P. fraxinifolium, Hiern.

Fl. Trop. Afr. iii. 22 ; Cat. Afr. Pl. Welw. i. 427.

Matopos, fl. and old fr. Sep. Gibbs, 83 ; Victoria Falls, Gibbs ; Allen, 4017; Bulawayo, Oct.-Dec. Chubb, 318; Victoria, Monro, 307. 
A Record of Plants Collected in Southern Rhodesia.

Genus No.

P. spp.

Mazoe, 4800 ft. March, Eyles, 268.

Salisbury, May, Flanagan, 3270. Same as Eyles 268, cf. Lefeburia abyssinica, Rich.

Victoria, Monro, 436, 524.

FAmily CCXXIX.-CORNACEAE, Link.

6156-Curtisia faginea, Ait.

Fl. Cap. ii. 570.

Chimanimani Mts. 7000 ft. fl. \& fr. Sep. Swyn. 637 ; Odzani River Valley, Umtali, Teague, 211.

\section{Subclass METACHLAMYDEAE.}

\section{SERIES ERICALES.}

FAmily CCXXXIII.-ERICACEAE, $D C$.

6237-Erica lanceolifera, S. Moore.

Journ. Linn. Soc. Bot. xl. 126.

Chimanimani Mts. 7000 ft. Sep. Swyn. 1288.

E. pleiotricha, S. Moore.

Journ. Linn. Soc. Bot. xl. 127.

Chimanimani Mts. 7000 ft. Sep. Swyn. 648a.

E. Swynnertonii, S. Moore.

Journ. Linn. Soc. Bot. xl. 128.

Melsetter, $6000 \mathrm{ft}$., between Lusitu and Nyahodi Rivs., $5000 \mathrm{ft}$., Chimanimani Mts. 6000 ft. fl. April, Sep. Swyn. 648, 1063, 1064, 1065.

E. thryptomenoides, S. Moore.

Journ. Linn. Soc. Bot. xl. 126.

Chimanimani Mts. 7000 ft. Sep. Swyn. 647.

6240-Philippia hexandra, S. Moore.

Journ. Linn. Soc. Bot. xl. 129.

Melsetter, 5000-6000 ft. Oct. Swyn. 1147; North Melsetter, Swyn. 6072.

P. milanjiensis, Britt. \& Rendle.

Mazoe, 5200 ft. April, Eyles, 336; Hunyani Riv. Rand, 501. 
P. Simii, S. Moore.

Journ. Linn. Soc. Bot. xl. 128.

Melsetter, 6000 ft. Oct. Swyn. 612.

\section{SERIES PRIMULALES.}

FAmily CCXXXVI.-MYRSINACEAE, Lindl.

6283-Maesa lanceolata, Forsk. (M. rufescens, A. DC.)

Fl. Trop. Afr. iii. 492 ; Fl. Cap. IV. i. 432 ; For. Fl. Cape, 249 ; Cat. Afr. Pl. Welw. i. 637.

Mazoe, 4300 ft. Jan. Eyles, 502 ; April, Eyles, 303 ; Salisbury, March, Flanagan, 3020; Chirinda, 3500 ft., North Melsetter, 5000-6000 ft. fl. \& fr. April, Sep. Oct. Swyn. 142, 172, 6074 ; also Inyamadzi Riv., Chikore Hills and Lusitu Valley, etc. Swyn.

M. sp.

Chirinda, Swyn. 669.

6310_Embelia nyassana, Gilg.

Chirinda, $3700 \mathrm{ft.} \mathrm{Swyn.} 120$.

E. oleifolia, S. Moore.

Journ. Bot. 1909, 297.

Bulawayo, Rand, 504 ; Monro, 31.

E. spp.

Salisbury, Rand, 558.

Victoria, Monro, 419.

6313-Myrsine africana, $L$.

Fl. Trop. Afr. iii. 493 ; Fl. Cap. IV. i. 434 ; Cat. Afr. Pl. Welw. i. 638 ; For. Fl. Cape, 250.

Melsetter, $6000 \mathrm{ft}$., Mt. Pene, $7000 \mathrm{ft}$., Chimanimani Mts. $7000 \mathrm{ft}$. Sep. Swyn. 633, 6153a, 6154a.

6314-Rapanea umbratilis, S. Moore.

Journ. Linn. Soc. Bot. xl. 130.

Melsetter, 6000 ft. Oct. Swyn. 6163.

FAmily CCXXXVII.-PRIMULACEAE, Vent.

6338--Anagallis Hanningtonii, Baker.

Chipetzana Riv. 3000 ft. fl. \& fr. Dec. Swyn. 357.

6340-Samolus valerandi, $L$.

Fl. Trop. Afr. iii. 490 ; Fl. Cap. IV. i. 430 ; Cat. Afr. Pl. Welw. i. 637.

Victoria Falls, Engler; Rogers, 5811. 
Family CCXXXVIII.-PLUMBAGINACEAE, Lindl.

Genus No.

6343-Plumbago zeylanica, $L$.

Fl. Trop. Afr. iii. 486; Fl. Cap. IV. i. 425 ; Cat. Afr. Pl. Welw. i. 634 .

Matopos, Sep. Gibbs, 14; Rogers, 5065 ; Bulawayo, March, Monro ; Rogers, 13610 ; Chubb, 209, 210 ; Victoria Falls, April, Flanagan, 3153 ; Salisbury, Rand, 505.

\section{Series EBENALES.}

Family CCXXXIX.-SAPOTACEAE, Dumort.

6368-Sideroxylon cinereum (Pierre), Torre \& Harms. (Pachystela cinerea, Pierre.)

Inyamadzi Valley, $3000 \mathrm{ft}$. Swyn. 571a.

6377-Chrysophyllum argyrophyllum, Hiern.

Cat. Afr. Pl. Welw. i. 641.

Mazoe, 4300 ft. fl. Sep. fr. Nov. Eyles, 378; Chimanimani Mts. 7000 ft. Sep. Nov. Swyn. 22b; also at Mt. Pene Forest and Inyamadzi Riv. Swyn.

C. fulyum, S. Moore.

Journ. Linn. Soc. Bot. xl. 131.

Chirinda Forest, 3700-4000 ft. fl. \& fr. Dec. Swyn. 19.

C. natalense, Sond.

Fl. Cap. IV. i. 437 ; Nat. Pl. 378; For. Fl. Port. E. Afr. 79 ;

For. Fl. Cape, 252.

Chipete Forest, Dec. Swyn. 21.

6386-Mimusops decorifolia, S. Moore.

Journ. Bot. 1911, 154.

Victoria, Monro, 811.

M. Kirkii, Baker.

Fl. Trop. Afr. iii. 507 ; For. Fl. Port. E. Afr. 80.

Victoria Falls, Rogers, 5117, 5310, 5672.

M. Mochisia, Baker.

Fl. Trop. Afr. iii. 506 ; For. Fl. Port. E. Afr. 80.

Victoria Falls, Galpin, 7052; Allen, 185.

M. Monroi, S. Moore.

Journ. Bot. 1911, 154.

Victoria, Monro, 761, 690. 
Genus No.

M. Zeyheri, Sond. var. laurifolia, Engl.

Fl. Cap. IV. i. 441 (type).

Victoria Falls, Galpin, 7044 ; Engler.

M. sp.

Mazoe, Govt. Herb. 2090.

Family CCXL.-EBENACEAE, Vent.

6403-Royena hirsuta, $L$.

Fl. Cap. IV. i. 451 ; For. Fl. Cape, 258.

Salisbury, Engler.

R. pallens, Thunb. (R. pubescens, Willd.)

Fl. Trop. Afr. iii. 510 ; Fl. Cap. IV. i. 453; For. Fl. Cape, 258 ; Nat. Pl. 232 ; Cat. Afr. Pl. Welw. i. 647.

Victoria Falls, Sep. Gibbs, 112 ; Allen, 177 ; Engler; Matopos, Gibbs ; Bulawayo, 4500 ft. Oct. Eyles, 1091 ; Monro, 39, 40 ; 113 ; Chubb, 378; Rogers, 5501, 13609 ; Chirinda, $3800 \mathrm{ft}$. Oct. Swyn. 62; Mt. Pene, 7000 ft. Swyn. 6070; South Rhodesia, Rand, 615.

R. villosa, $L$.

Fl. Cap. IV. i. 450 ; For. Fl. Cape, 257 ; Nat. Pl. 201.

Umtali, Engler.

6404-Euclea divinorum, Hiern.

Fl. Trop. Afr. iii. 513 ; Fl. Cap. IV. i. 469 ; For. Fl. Port. E. Afr. 81.

Victoria Falls, Kirk; Matopos, Sep. Gibbs, 34 ; Galpin, 7078 ; Gwelo, Sr. Phil. 50.

E. Eylesii, Hiern.

Journ. Bot. 1907, 47.

Sebakwe, 4000 ft. Dec. Eyles, 44 ; Victoria, Monro, $643 b$.

E. Kellau, Hochst.

Fl. Trop. Afr. iii. 514.

Bulawayo, $4500 \mathrm{ft}$. Nov. Eyles, 1252.

E. lancea, Thunb.

Fl. Cap. IV. i. 464 ; For. Fl. Cape, 260.

Matopos, Engler.

E. lanceolata, E. Mey.

Fl. Trop. Afr. iii. 512 ; Fl. Cap. IV. i. 467 ; Cat. Afr. Pl. Welw. i. 648 ; For. Fl. Cape, 261.

Bulawayo, Monro, 42, 43 ; Victoria, Monro, 517; Matopos, Galpin, 7005 ; Chirinda, $3500 \mathrm{ft}$. Swyn. 743 ; Chipete Forest, Swyn. 1298; Umvumvumvu Riv. 2000 ft. Swyn. 1305 ; in fl. April, Sep. Swyn.; South Rhodesia, Rand, 131, 361. 
Genus No.

var. angustifolia, Hiern.

Mazoe, $5300 \mathrm{ft}$. + Nov. Eyles, 470.

var. confertiflora, Hiern.

Mazoe, $4200 \mathrm{ft}$. $\precsim$ Sep. Eyles, 410.

var. parvifolia, Hiern.

Mazoe, 5000 ft. ð Nov. Eyles, 457.

E. macrophylla, E. Mey.

Fl. Cap. IV. i. 472 ; For. Fl. Cape, 262.

Victoria Falls, Engler.

E. multiflora, Hiern.

Fl. Trop. Afr. iii. 513 ; Fl. Cap. IV i. 470 ; Cat. Afr. Pl. Welw. i 649 ; For. Fl. Cape, 262.

Matopos, ð Sep. Gibbs, 46 ; Victoria, Monro, 423, 419 ; Odzani River Valley, Umtali, Teague, 237 ๘.

E. undulata, Thunb.

Fl. Cap. IV. i. 474 ; For. Fl. Cape, 263.

Matopos, Davy ; Lomagundi, Govt. Herb. 973.

\section{E. spp.}

Victoria Falls, Allen, 161.

Matopos, Sep. Galpin, 7070 cf. E. natalensis, A. DC.

Salisbury, Rand, 563.

Leopard Mine, Govt. Herb. 942.

6405-Maba Mualala, Welw.

Fl. Trop. Afr. iii. 515.

Chirinda Forest, 3700-4000 ft. Jan. Swyn. 3.

6406-Diospyros mashuma?. (Nоте.-Swyn. gives native name of D. sabiensis, Hiern, as "Mashuma.")

Matopos, Govt. Herb. 886 ; Lomagundi, Govt. Herb. 980.

D. mespiliformis, Hochst.

Fl. Trop. Afr. iii. 518; Fl. Cap. IV. i. 477 ; Cat. Afr. Pl. Welw. i. 651 ; For. Fl. Port. E. Afr. 83.

Victoria Falls, Rogers, 5118; South Umtali, 2000-3000 ft. fr. Oct. Swyn. 6071; Umvumvumvu Riv. 4000 ft. Dec. Swyn. 6622 ; Victoria, Monro, 391, 713.

D. sabiensis, Hiern.

Journ. Linn. Soc. Bot. xl. 134.

Sabi Riv. 1000 ft. Nov. Swyn. 1209.

D. spp.

Gwanda, Noble, 6.

Victoria Falls, Sep. Allen, 395.

Victoria, Monro, 413. 


\section{Series CONTORTAE.}

\section{Family CCXLIII.-OLEACEAE, Lindl.}

Genus No.

6422-Schrebera mazoensis, S. Moore.

Journ. Bot. 1907, 48.

Mazoe, 4300-5300 ft. Dec. Eyles, 202 ; Marandellas, Mundy.

\section{S. spp.}

Victoria Falls, Allen, 148 cf. S. golungensis, Welw.

Victoria, Monro, 675, 978.

6434-Olea chrysophylla, Lam.

Fl. Trop. Afr. IV. i. 18.

Victoria, Monro, 367, 744.

0. laurifolia, Lam.

Fl. Cap. IV. i. 487 ; For. El. Cape, 264.

Victoria, Monro, 404 ; Matopos, Govt. Herb. 892.

0. verrucosa, Link. (O. woodiana, Knobl.)

Fl. Cap. IV. i. 486 ; Nat. Pl. 237 ; For. Fl. Port. E. Afr. 83 ; For. Fl. Cape, 266.

Gwanda, Noble, 7 ; Matopos, Govt. Herb. 888, 889 ; Victoria Falls, Rogers, 5557 ; Chimanimani Mts. 7000 ft. bud Sep. Swyn. 1281.

0. sp.

Matopos, Govt. Herb. 893.

6435-Dekindtia africana, Gilg.

Fl. Trop. Afr. IV. i. 588.

Chirinda, 3800 ft. Oct. Swyn. 171.

6438-Menodora heterophylla, Moric.

Fl. Cap. IV. i. 484.

South Rhodesia, Rand, 595.

6440-Jasminum mauritianum, Bojer.

Fl. Trop. Afr. IV. i. 10 ; Fl. Cap. IV. i. 482 ; Cat. Afr. Pl. Welw. i. 655 .

Victoria Falls, Sep. Galpin, 7020 ; fl. \& fr. Sep. Gibbs, 111 ;

Allen, 178; Rogers, 5155; Engler; Mazoe, 4200 ft. Jan.

Eyles, 246; Sabi Riv. 1000 ft. Nov. Swyn. 2033.

J. oleæcarpum, Baker.

Fl. Trop. Afr. IV. i. 8.

Gwelo, Jan. Gardner, 21. 
Genus No. stenolobum, Rolfe.

Fl. Trop. Afr. IV. i. 4 ; Fl. Cap. IV. i. 481.

Matabeleland, Oates; Elliott; Matopos, Cecil, 113; Gwelo, Cecil, 132; Victoria Falls, Allen, 37; Rogers, 5493; Bulawayo, Dec. Gardner, 85; Monro, 4 ; Mazoe, $4400 \mathrm{ft}$. Nov. Eyles, 443; Victoria, Monro, 514, 515; Sabi Riv. 1000 ft. Nov. Swyn. 2034 ; South Rhodesia, Rand, 125.

\section{J. spp.}

Bulawayo, $4500 \mathrm{ft}$. Dec. Eyles, 55.

Victoria, Monro, 746, 836.

\section{FAmily CCXLV.-LOGANIACEAE, Lindl.}

6449-Mostuea Walleri, Baker.

Fl. Trop. Afr. IV. i. 507.

Chirinda Forest, 3800 ft. Dec. Swyn. 6511.

6460-Strychnos Burtoni, Baker.

Fl. Trop. Afr. IV. i. 533.

Chirinda, Dec. Swyn. 1959; also Chipetzana and between Chirinda and Chipinga, Swyn.

S. matopensis, S. Moore.

Journ. Bot. 1905, 48.

Matopos, $4500 \mathrm{ft}$. Nov. Eyles, 1182.

S. mellodora, S. Moore.

Journ. Linn. Soc. Bot. xl. 147.

Chirinda Forest, 3700-4000 ft. Oct. Swyn. 101.

S. micans, S. Moore.

Journ. Linn. Soc. Bot. xl. 146.

Chirinda Forest, 3700-4000 ft. Dec. Swyn. 125.

S. mitis, S. Moore.

Journ. Linn. Soc. Bot. xl. 146.

Chirinda Forest, 3700-4000 ft. fl. Jan. fr. Oct. Swyn. 17, $17 a$.

S. pungens, Solered.

Fl. Trop. Afr. IV. i. 530 ; Fl. Cap. IV. i. 1051; Cat. Afr. Pl. Welw. i. 704.

Matopos, Davy; North of Bulawayo, Engler.

S. spinosa, Lam.

Fl. Trop. Afr. IV. i. 536 ; Fl. Cap. IV. i. 1055 ; Cat. Afr. Pl. Welw. i. 702 ; For. Fl. Port. E. Afr. 89 ; For. Fl. Cape, 274. Chirinda, 3800 ft. Oct. Swyn. 55, 55a; throughout South Melsetter Dist.; South Rhodesia, Rand, 426; Victoria Falls, Rogers, 5976*, 7237*. 
S. tonga, Gilg.

Salisbury, Engler.

S. sp.

Lomagundi, Govt. Herb. 983.

6466-Anthocleista zambesiaca, Baker.

Fl. Trop. Afr. IV. i. 540 ; Fl. Cap. IV. i. 1049.

Chirinda, Swyn.

6469-Nuxia dentata, $R . B r$.

Fl. Trop. Afr. IV. i. 513 ; Fl. Cap. IV. i. 1040 ; Cat. Afr. Pl. Welw. i. 700.

Odzani Riv. Cecil, 235 ; Lusitu Riv. 3000 ft. Nov. Swyn. 1294 ; Victoria, Monro, 497, 605, 748, 894.

N. Holstii, Gilg.

Fl. Trop. Afr. IV. i. 515.

Melsetter, April, Swyn. 1902.

N. sambesina, Gilg.

Fl. Trop. Afr. IV. i. 514.

Melsetter, $6000 \mathrm{ft}$. Sep. Swyn. 606.

N. viscosa, Gibbs.

Journ. Linn. Soc. Bot. xxxvii. 454.

Matopos, Oct. Gibbs, 246 ; Victoria, Monro, 435, 735.

6470-Gomphostigma scoparioides, Turcz.

Bulawayo, $4500 \mathrm{ft}$. Dec. Eyles, 14; Tengwe Riv. Baines.

6471-Chilianthus arboreus, A. DC. (C. oleaceus, Burch.)

Fl. Cap. IV. i. 1043 ; For. Fl. Cape, 276.

Bulawayo, $4500 \mathrm{ft}$. Dec. Eyles, 1138.

6473-Buddleia salvifolia, Lam.

Fl. Trop. Afr. IV. i. 516 ; Fl. Cap. IV. i. 1046 ; For. Fl. Cape, 277.

Bulawayo, Monro, 77 ; Melsetter, 6000 ft. Sep. Swyn. 609, 675 ; Mt. Pene, 6500-7000 ft. Oct. Swyn. 6090.

Family CCXLVI.-GentianaCEAE, Dumort.

6479-Exacum quinquenervium, Griseb.

Fl. Trop. Afr. IV. i. 546.

Victoria Falls, Rogers, 7101.

6481-Sebæa barbeyiana, Schinz.

Fl. Trop. Afr. IV. i. 549.

Victoria Falls, Kirk; Allen, 40.

S. leiostyla, Gilg.

Fl. Trop. Afr. IV. i. 548 ; Fl. Cap. IV. i. 1086.

Melsetter, 6000 ft. April, Swyn. 1898. 
Genus No.

6483-Exochænium, in place of Belmontia, after A. W. Hill, in Kew Bull. 1908, pp. 317-341.

Exochænium exiguum, A. W. Hill. (Chironia exigua, Oliv.)

Kew Buil. 1909, 50.

Matopos, $4500 \mathrm{ft}$. March, Eyles, 1032.

E. grande, Griseb. (Belmontia grandis, E. Mey. ; Parasia grandis, Hiern.)

Fl. Trop. Afr. IV. i. 553 ; Fl. Cap. IV. i. 1094; Cat. Afr. Pl Welw. i. 707 ; Harv. Gen. S.A. Pl. 252.

Mazoe, $4400 \mathrm{ft}$. April, Eyles, 319 ; Chipetzana source, $4000 \mathrm{ft}$. April, Swyn. 2031.

6500-Canscora diffusa (Vahl.), R. Br.

Fl. Trop. Afr. IV. i. 558.

Victoria Falls, Engler.

C. Kirkii, $N . E . B r$.

Fl. Trop. Afr. IV. i. 558.

Victoria Falls, Kirk; Rogers, 5043 ; fl. \& fr. Sep. Gibbs, 152 ; Allen, 413 ; July, Kolbe, 3147 ; Matopos, Rogers.

6503-Chironia gratissima, S. Moore.

Journ. Linn. Soc. Bot. xl. 148.

Melsetter, 6000 ft. Sep. Swyn. 1892; North Melsetter, 5000-6000 ft. Oct. Swyn. 6162.

C. humilis, Gilg.

Fl. Cap. IV. i. 1107.

Matopos, Nov. Eyles, 1143 ?; Victoria, Monro, 756 ?

var. Wilmsii, Prain. (C. humilis, Bak. \& Br.; C. purpurascens, Rolfe.)

Fl. Cap. IV. i. 1107 ; Fl. Trop. Afr. IV. i. 555 ; see Kew Bull. 1908, pp. 350, 373, 375.

South Rhodesia, Rand, 168; Matabeleland, Oates; Inyanga, 6000-7000 ft. Cecil, 190.

C. transvaalensis, Gilg. (C. palustris, Gilg.)

Fl. Trop. Afr. IV. i. 555 ; Fl. Cap. IV. i. 1105.

Umtali, Cecil, 162; Victoria Falls, Allen, 208; Gwelo, Jan. Gardner, 46; Mazoe, 4400 ft. Feb. Eyles, 527; Victoria, Monro, 662.

C. spp.

Bulawayo, Eyles, 90.

Gwai, 3000 ft. Jan. Allen, 228.

6512-Sweertia stellarioides, Ficalho. (S. Welwitschii, Engl.)

Fl. Trop. Afr. IV. i. 581; Fl. Cap. IV. i. 1119 ; Cat. Afr. Pl. Welw. i. 711.

Salisbury, March, Flanagan, 3019 ; Melsetter, 6000 ft. April, Swyn, 1919 ; Odzani River Valley, Umtali, Teague, 99. 
Genus No.

6545-Limnanthemum indicum, Dur. \& De Wild.

Fl. Trop. Afr. IV. i. 587.

South Rhodesia, Rand, 173.

L. thunbergianum, Griseb.

Fl. Trop. Afr. IV. i. 584 ; Fl. Cap. IV. i. 1120 ; Nat. Pl. 34.

Victoria Falls, Allen, 82 ; Upper Umswirizwi, 2600 ft. fl. \& fr. Oct. Swyn. 393.

Family CCXLVII.-APOCYNACEAE, Lindl.

6558-Acocanthera venenata, G. Don.

Fl. Trop. Afr. IV. i. 94 ; Fl. Cap. IV. i. 501 ; For. Fl. Cape, 270.

Bulawayo, Rand, 572 ; Matopos, Sep. Galpin, 7076.

6559-Carissa Arduina, Lam. (C. acuminata, DC.)

Fl. Trop. Afr. IV. i. 91; Fl. Cap. IV. i. 498; Nat. Pl. 203 ; For. Fl. Port. E. Afr. 86 ; For. Fl. Cape, 269.

Bulawayo, Rand, 285 ; Matopos, Davy ; Chirinda Forest, 37004000 ft. Swyn.; Mt. Pene, 7000 ft. Swyn., fl. Sep. Dec. Swyn.

C. edulis, Vahl.

Fl. Trop. Afr. IV. i. 89 ; For. Fl. Port. E. Afr. 86.

Victoria Falls, Kirk; Davy.

var. tomentosa, Stapf. (C. tomentosa, A. Rich.)

Fl. Trop. Afr. IV. i. 90 ; Fl. Cap. IV. i. 497.

Matabeleland; Oates; Bulawayo, Rand, 176; Engler; Khami, Nov. Marloth, 3393; Matopos, Davy; Engler ; Govt. Herb. 885;

Victoria Falls, Davy; Bulawayo, 4500 ft. Sep. Eyles, 23 ;

Chubb, 314; Rogers, 5509; Mrewa, Govt. Herb. 865; Chirinda, 3800 ft. Swyn. 70 ; South Umtali Dist. 2000-3000 ft.

Swyn. 6621, 6623 ; fl. Oct. Dec. Swyn.; Victoria, Monro, 450.

6562-Landolphia Buchananii, Stapf.

Fl. Trop. Afr. IV. i. 35 ; For. Fl. Port. E. Afr. 85.

Umtali, Engler.

L. Kirkii, Dyer.

Fl. Trop. Afr. IV. i. 55 ; For. Fl. Port. E. Afr. 85.

Chirinda, 3800 ft. Oct. Dec. Swyn. 81a; South Melsetter, Govt. Herb. 716.

L. (?) lucida, $K$. Schum.

Fl. Trop. Afr. IV. i. 59.

Chirinda Forest, 3700 ft. Feb. Swyn. 83.

L. Swynnertonii, S. Moore.

Journ. Linn. Soc. Bot. xl. 136.

Chirinda Forest, 3700-4000 ft. Nov. Swyn. 82 ; Chipete, Swyn. 
A Record of Plants Collected in Southern Rhodesia.

Genus No.

L. sp.

Victoria Falls, Engler.

6563-Clitandra orientalis, $K$. Schum.

Fl. Trop. Afr. IV. i. 594.

Victoria Falls, Rogers, 7234*, 7491*, 7505*.

6577-Pleiocarpa Swynnertonii, S. Moore.

Journ. Linn. Soc. Bot. xl. 138.

Chirinda Forest, fl. Sep. fr. Dec. Swyn. $14 \& 6503$.

6581-Gonioma Kamassi, E. Mey.

Fl. Cap. IV. i. 503 ; For. Fl. Cape, 272.

Gwanda, Noble, 16 ; Lomagundi, Govt. Herb. 995.

6582-Holarrhena febrifuga, Klotzsch.

Fl. Trop. Afr. IV. i. 162.

South Melsetter, Swyn.; Victoria, Monro, 809.

6589-Diplorrhynchus mossambicensis, Benth.

Fl. Trop. Afr. IV. i. 107 ; For. Fl. Port. E. Afr. 86.

Matopos, Oct. Gibbs, 273 ; Govt. Herb. 890 ; Engler; Chikore

Hills, fr. April, Swyn.; Umswirizwi Riv. Swyn.; Victoria

Falls, Rogers, 7221*.

6597-Lochnera rosea, Reichb. (Vinca rosea, L.)

Fl. Trop. Afr. IV. i. 118 ; Fl. Cap. IV. i. 504.

Salisbury, Darling.

6605-Conopharyngia elegans, Stapf.

Fl. Trop. Afr. IV. i. 149; Fl. Cap. IV. i. 506; For. Fl. Port. E. Afr. 87.

Chikore Hills, Swyn.

C. stapfiana, Stapf.

Fl. Trop. Afr. IV. i. 147.

Mt. Pene, 7000 ft., Oct. Swyn. 6148, 560.

C. usambarensis, Stapf.

Fl. Trop. Afr. IV. i. 148.

Chirinda Forest, 3700-4000 ft. fl. \& young fr. Oct. Swyn. 37 $\& 37 a$.

6619-Rauwolfia caffra, Sond.

Fl. Trop. Afr. IV. i. 110 ; Fl. Cap. IV. i. 502.

Bulawayo, Govt. Herb. 1081; Mashonaland, Govt. Herb. 1000.

R. inebrians, K. Schum.

Fl. Trop. Afr. IV. i. 112.

Chirinda Forest, 3700-4000 ft.; Sep. Nov. Swyn. 6 ; Inyamadzi

Valley, 3000 ft. Swyn. 602 ; Melsetter, 5000 ft. Swyn. ; Mt.

Pene Forest, Swyn.

R. sp.

Victoria Falls, Engler. 
Genus No.

6632-Thevetia nerifolia?.

Matopos, Govt. Herb. 898 (cultivated).

6680-Adenium multiflorum, Kiotzsch.

Fl. Trop. Afr. IV. i. 229 ; Fl. Cap. IV. i. 514 ; For. Fl. Port. E. Afr. 89.

Sabi Riv. 1000 ft. fr. Nov. Swyn. 557.

6686-Oncinotis chirindica, S. Moore.

Journ. Linn. Soc. Bot. xl. 141.

Chirinda Forest, 3700-4000 fl. \& fr. Oct. Swyn. 87; Chipete, Swyn.

Family CCXLVIII.-ASCLEPIADACEAE, Lindl.

6688-Strophantus sp.

Wankie, Rogers, 58370 (near S. arnoldianus, Wildern \& Dur.) 6729-Chlorocodon Whytei, Hook. $f$.

Fl. Trop. Afr. IV. i. 255 ; Fl. Cap. IV. i. 542 ; Cat. Afr. Pl. Welw. i. 680 ; Nat. Pl. 31.

Mazoe, 4300 ft. Dec. Eyles, 204 ; Victoria Falls, Allen, 259.

6730-Tacazzea Kirkii, N. E. Br.

Fl. Trop. Afr. IV. i. 268; Fl. Cap. IV. i. 540.

Victoria Falls, Allen, 252, 283; Rogers, 5091, 5566, 7403;

Victoria, Monro, 1099, 797.

6740-Crytolepsis oblongifolia, Schlechter. (Ectadiopsis oblongifolia, Benth.)

Fl. Trop. Afr. IV. i. 249 ; Fl. Cap. IV. i. 529; Nat. Pl. 513.

Mazoe, 5000-5300 ft. Nov. Eyles, 469 ; Bulawayo, Monro, 109 ;

Victoria, Monro, 752 ; Chirinda, fl. \& fr. Oct. Swyn. 243.

C. producta, N. E. Br.

Fl. Trop. Afr. IV. i. 247.

Gwai Forest, 3600 ft. Allen, 239 ; Salisbury, Rand, 1368.

C. transvaalensis, Schlechter.

Fl. Cap. IV. i. 528.

Victoria, Monro, 812.

6747-Raphiacme lanceolata, Schinz, var. latifolia, N. E. Br.

Fl. Trop. Afr. IV. i. 274 (Raphionacme).

Bulawayo, Rand, 284 ; Monro, 94.

R. longifolia, N. E. Br.

Fl. Trop. Afr. IV. i. 274.

Mazoe, $5300 \mathrm{ft}$. Nov. Eyles, 468.

R. procumbens, Schlechter.

Fl. Cap. IV. i. 535.

Bulawayo, Monro, 111. 
Genus No.

\section{R. spp.}

Gwelo, Jan. Gardner, 13.

Bulawayo, Monro, 100.

6777-Xysmalobium bellum, N. E. Br.

Fl. Trop. Afr. IV. i. 311.

Mazoe, 4800 ft. Dec. Eyles, 492.

X. Cecilae, $N$. E. Br.

Fl. Trop. Afr. IV. i. 310.

Salisbury, Cecil, 60 ; Rand, 1358; Victoria, Monro.

X. dispar, N. E. Br.

Fl. Trop. Afr. IV. i. 307.

Mazoe, 4400 ft. Feb. Eyles, 529.

X. gramineum, S. Moore.

Fl. Trop. Afr. IV. i. 302.

Bulawayo, Rand, 193.

X. recticulatum, $N . E . B r$.

Fl. Trop. Afr. IV. i. 303.

Mazoe, 4200 ft. Nov. Eyles, 467 ; between Umtali and Salisbury, Cecil, 51 ; between Enkeldoorn and Rocky Spruit, Cecil, 135 ; Malindi, Gwai Forest, Allen, 227; Chirinda, 3800 ft. Oct. Swyn. 247; Victoria, Monro, 628.

X. sp.

Bulawayo, Chubb, 7a, cf. X. decipiens, N. E. Br.

6778-Schizoglossum aciculare, N. E. Br.

Fl. Trop. Afr. IV. i. 363 ; Fl. Cap. IV. i. 620.

Salisbury, Rand, 1359.

S. biflorum, Schlechter.

Fl. Cap. IV. i. 641.

Victoria, Monro, 657.

var. gwelense, N. E. Br. (S. gwelense, N. E. Br.)

Fl. Cap. IV. i. 642 ; Fl. Trop. Afr. IV. i. 360.

Gwelo, Cecil, 131 ; Salisbury, Rand, 190, 1354.

S. Carsoni, N. E. Br.

Fl. Trop. Afr. IV. i. 366.

Between Umtali and Salisbury, Cecil, 48; Selukwe, Cecil, $48 a$;

Salisbury, Darling; Rand, 1361 ; Mazoe, $4800 \mathrm{ft.}$

Dec. Eyles, 216.

S. chirindense, S. Moore.

Journ. Bot. 1908, 295.

Chirinda, $3800 \mathrm{ft}$. Swyn. 246, Mt. Pene, $7000 \mathrm{ft}$. in fl. Sep. Oct. Swyn. 246, 6095. 
Genus No.

S. Eylesii, S. Moore.

Journ. Bot. 1914, 149.

Mazoe, $4800 \mathrm{ft}$. Jan. Eyles, 500.

S. Pentheri, Schlechter.

Eyles, 1116.

S. strictissimum, S. Moore.

Fl. Trop. Afr. IV. i. 358.

Bulawayo, Rand, 195 ; Victoria, Monro, 842.

6780-Kanahia glaberrima, N. E. Br.

Fl. Trop. Afr. IV. i. 297.

Shashi Riv. Baines.

K. Monroi, Moore.

Journ. Bot. 1911, 156.

Victoria, Monro, 1100.

6781-Margaretta Holstii, K. Schum.

Fl. Trop. Afr. IV. i. 374.

Victoria Falls, Allen, 194.

M. Whytei, K. Schum.

Fl. Trop. Afr. IV. i. 374.

Salisbury, Rand, 124, 547, 632 ; March, Flanagan, 2999 ; Darling in Herb. Bolus 11192; Rand, 1360; Rogers, 4008; Mazoe, 4300 ft. Sep. Eyles, 428; Chirinda, 3800 ft. Oct. Swyn. 1912.

6783-Cordylogyne kassnerianum (Schlechter), Torre \& Harms. (Periglossum kassnerianum, Schlechter.)

Fl. Cap. IV. i. 584.

Victoria, Monro, 624.

C. mossambicense (Schlechter), Torre \& Harms. (Periglossum mossambicense, Schlechter.)

Fl. Cap. IV. i. 583.

Salisbury, Rand, 1355.

6787-Gomphocarpus concolor, Decne. (Pachycarpus concolor, E. Mey.)

Fl. Trop. Afr. IV. i. 377 ; Fl. Cap. IV. i. 729.

Bulawayo, Rand, 187 ; Rogers, 5930 ; Salisbury, Rand, 1356.

6791-Asclepias aurea, Schlechter. (Gomphocarpus aureus, Schltr.)

Fl. Trop. Afr. IV. i. 345; Fl. Cap. IV. i. 685.

Salisbury, Rand, 638 ; Cecil, 144 ; Gwelo, Rand, 188; Matopos, 5000 ft. Nov. Eyles, 1152 ; Victoria, Monro, 313.

A. densiflora, N.E. Br.

Fl. Trop. Afr. IV. i. 320 ; Fl. Cap. IV. i. 705.

Between Salisbury and Bulawayo, Cecil, 78. 
6791-亡̈. eminens, Schlechter.

Fl. Trop. Afr. IV. i. 351 ; Fl. Cap. IV. i. 685.

Between Enkeldoorn and Rocky Spruit, Cecil, 134; Bulawayo, Rand, 183, 189 ; Gwelo, Jan. Gardner, 52 ; Mazoe, 4200 ft. Nov. Eyles, 460 ; Victoria, Monro, 618.

A. Engleri, Schlechter.

Umtali, Engler.

A. fallax, Schlechter.

Fl. Cap. IV. i. 706.

Salisbury, Rand, 1352 ; Bulawayo, Monro, 19.

A. fruticosa, $L$. (Gomphocarpus fruticosus, R. Br.)

Fl. Trop. Afr. IV. i. 330 ; Fl. Cap. IV. i. 691.

Shangani Riv. Rand, 191 ; Shashi Riv. Baines; Matopos, fl. \& fr. Oct. Gibbs, 103 ; Victoria Falls, Allen, 275; Salisbury, Govt. Herb. 921; Victoria, Monro, 913.

A. fulva, N.E. Br.

Fl. Trop. Afr. IV. i. 321.

Chirinda, 3600 ft. Jan. Swyn. 1914.

A. glaucophylla, Schlechter. (Gomphocarpus glaucophyllus, Schlechter.)

Fl. Trop. Afr. IV. i. 321 ; Fl. Cap. IV. i. 696.

Salisbury, Cecil, 57 ; Rand, 185, 1357 ; Charter, Govt. Herb. 3023.

A. lineolata, Schlechter. (Gomphocarpus lineolatus, Decne.)

Fl. Trop. Afr. IV. i. 322.

Bulawayo, Rand, 184; Salisbury, Rand, 1353; Cecil, 66 ; Sebakwe, 4000 ft. Dec. Eyles, 166 ; Victoria Falls, Allen, 86 ; Upper Buzi, 3200 ft. Nov. Swyn. 245.

A. palustris, Schlechter.

Fl. Trop. Afr. IV. i. 349.

Mazoe, 4300-4800 ft. Nov. Eyles, 448.

A. physocarpa, Schlechter. (Gomphocarpus physocarpus, E. Mey.) Fl. Trop. Afr. IV. i. 328 ; Fl. Cap. IV. i. 692 ; Nat. Pl. 217. Eyles, 1129.

A. Randii, S. Moore.

Fl. Trop. Afr. IV.i. 351.

Salisbury, Rand, 194.

A. reflexa, Britten \& Rendle.

North of Umtali, Cecil, 160 ; Sebakwe, $4000 \mathrm{ft}$. Dec.

Eyles, 169.

A. scabrifolia, S. Moore.

Journ. Bot. 1908, 297.

Chimanimani Mts. 7000 ft. Swyn. 1915; Mt. Pene, $7000 \mathrm{ft}$. Swyn. 6094, 6140, fl. Sep. Oct. Swyn. 
Genus No.

Ä. Swynnertonii, S. Moore.

Journ. Linn. Soc. Bot. xl. 142.

Mt. Pene, 7000 ft. Oct. Swyn. 6092 ; Melsetter, $6000 \mathrm{ft.} \mathrm{Swyn.}$ 6093.

A. tenuifolia, N. E. Br.

Fl. Trop. Afr. IV. i. 337.

Mangwe, Baines; Matopos, 5000 ft. March, Eyles, 1011; Sep. Gibbs, 100 ; March, Flanagan, 2989 ; Victoria, Monro, 422, 754, 1065; South Rhodesia, Rand, 192.

\section{A. spp.}

Salisbury, Darling in Herb. Bolus, 10796.

Matopos, Eyles, 1156.

6810-Pentarrhinum insipidum, E. Mey.

Fl. Trop. Afr. IV. i. 378; Fl. Cap. IV. i. 741 ; Nat. Pl. 517 ; Cat. Afr. Pl. Welw. i. 687.

Bulawayo, 4500 ft. Feb. Eyles, 106.

6834-Cynanchum chirindense, S. Moore.

Journ. Bot. 1908, 305.

Chirinda Forest, 3900 ft. Feb. Swyn. 137.

C. pracox, Schlechter.

Fl. Trop. Afr. IV. i. 399.

Mazoe, Rand, 512.

C. sp.

South Rhodesia, Rand, 362.

6849-Sarcostemma viminale, $R . B r$.

Fl. Trop. Afr. IV. i. 384 ; Fl. Cap. IV. i. 755 ; Cat. Afr. Pl. Welw. i. 689.

Matopos, Cecil, 106 ; Oct. Gibbs, 239 ; Engler ; Nov. Eyles, 1155 ?; Bulawayo, Rand, 363 ; Chubb, 1 a.

6860-Secamone Alpini, Schult.

Fl. Trop. Afr. IV. i. 279 ; Fl. Cap. IV. i. 544.

Chirinda, 3700-4000 ft. Dec. Swyn. 88; Bulawayo, Rogers, 13576 *.

6862-Oranthera jasminiflora, N. E. Br.

Fl. Trop. Afr. IV. i. 434.

Victoria Falls, Rogers, 7468*.

6865-Macropetalum Burchellii, Decne.

Fl. Cap. IV. i. 798 ; Harv. Gen. S.A. Pl. 241.

Fig-tree, $4500 \mathrm{ft}$. Dec. Eyles, 155.

6870-Brachystelma Barberiae, Harv.

Fl. Cap. IV. i. 864 ; Nat. Pl. 587.

Bulawayo, March, Monro. 
Genus No

6874 Ceropegia abyssinica, Decne.

Fl. Trop. Afr. IV. i. 462.

Salisbury, Rand, 1400.

C. hispidipes, S. Moore.

Journ. Bot. 1908, 309.

Chirinda, 3800 ft. Feb. Swyn. 1137.

C. mazoensis, S. Moore.

Journ. Bot. 1908, 309.

Mazoe, 4700 ft. Jan. Eyles, 518.

C. tentaculata, N. E. Br.

Fl. Trop. Afr. IV. i. 443 ; Cat. Afr. Pl. Welw. i. 694.

Victoria, Monro, 834, 889.

C. sp.

Salisbury, Ranđ̄, 1399.

6875--Riocreuxia profusa, N. E. Br.

Fl. Trop. Afr. IV. i. 465.

Chikore Hills, 3500 ft. April, Swyn. 205.

6880-Decabelone elegans, Decne. (Tavaresia angolensis, Welw.)

Fl. Trop. Afr. IV. i. 493 ; Cat. Afr. Pl. Welw. i. 697.

South Rhodesia, Rand, 286.

6884-Caralluma lateritia, N. E. Br.

Fl. Trop. Afr. IV. i. 486.

Bulawayo, 4500 ft. Feb. Eyles, 22.

6885-Stapelia gigantea, N. E. Br.

Fl. Trop. Afr. IV. i. 501 ; Fl. Cap. IV. i. 948 ; Nat. Pl. 531.

Bulawayo, Monro.

S. Marlothii, N. E. Br.

Kew Bull. 1908, 436.

Matopos, Marloth, 3414.

6887-Heurnia sp.

Salisbury, Darling in Herb. Bolus 10767.

6891-Gymnema sylvestre, $R . B r$.

Fl. Trop. Afr. IV. i. 413 ; Fl. Cap. IV. i. 782.

Victoria Falls, Rogers, 5731.

6911-Marsdenia zambesiaca, Schlechter.

Fl. Trop. Afr. IV. i. 420.

Victoria Falls, Allen, 84.

6911a-Swynnertonia cardinea, S. Moore.

Journ. Bot. 1908, 308.

Chirinda Forest, 3700-4000 ft. Oct. Dec. Swyn. 1080, 6518.

6924-Fockea Monroi, S. Moore.

Journ. Bot. 1914, 149.

Victoria, Monro, 828, 837. 


\section{Series TUBIFLORAE.}

Family CCXliX.-CONVOLVUlaCEAE, Vent.

Genus No.

6968 - Cuscuta kilimanjari, Oliv.

Fl. Trop. Afr. IV. ii. 205.

Chirinda, 3800 ft. May, Swyn. 453, on Mellera lobulata, S. Moore.

C. obtusiflora, var. cordofana, Engelm.

Fl. Trop. Afr. IV. ii. 204.

Mazoe, 4300 ft. April, Eyles, 352 ; Govt. Herb. 2081.

C. planiflora, Ten.

Fl. Trop. Afr. IV. ii. 203 ; Cat. Afr. Pl. Welw. i. 743.

Mazoe, $5000 \mathrm{ft}$. April, Eyles, 327.

6973--Evolyulus alsinoides, $L$.

Fl. Trop. Afr. IV. ii. 67 ; Fl. Cap. IV. ii. 79 ; Cat. Afr. Pl. Welw. i. 724.

Matabeleland, Oates; Elliott; Cecil, 116; Rand, 127 ; Victoria Falls, Allen, 124; April, Flanagan, 3156; Bulawayo, Dec.

Gardner, 90 ; Rogers, 5758; Odzani River Valley, Teague, 262.

E. spp.

Bulawayo, $4500 \mathrm{ft}$. Jan. Eyles, 1.

6978-Seddera capensis, Hallier f. (Breweria capensis, Baker.)

Fl. Trop. Afr. IV. ii. 77 ; Fl. Cap. IV. ii. 80.

Salisbury, Rand, 126 ; Matabeleland, Gardner.

6991-Jacquemontia capitata, G. Don.

Fl. Trop. Afr. IV. ii. 85 ; Fl. Cap. IV. ii. 69 ; Nat. Pl. 13 ; Cat. Afr. Pl. Welw. i. 725.

Wankie, 2500 ft. May, Eyles, 129 ; Victoria Falls, Rogers, 7004.

6993-Convolvulus Randii, Rendle.

Fl. Trop. Afr. IV. ii. 94.

Gwelo, Rand, 274.

C. sagittatus, var. abyssinica, Hallier $f$.

Fl. Trop. Afr. IV. ii. 96.

Salisbury, Rand, 510 ; Cecil, 74 ; Chirinda, 3500 ft. June, Swyn. 1798.

C. ulosepalus, Hallier $f$.

Fl. Trop. Afr. IV. ii. 95 ; Fl. Cap. IV. ii. 73.

Salisbury, Engler.

C. $\mathrm{sp}$.

Mazoe, 5000 ft. Nov. Eyles, 1106. 
A Record of Plants Collected in Southern Rhodesia.

Genus No.

6995-Hewittia bicolor, Wight. (Shutereia bicolor, Choisy.)

Fl. Cap. IV. ii. 68 ; Cat. Afr. Pl. Welw. i. 727 ; Nat. Pl. 281.

Chirinda, 3700-4000 ft. June, Swyn. 1187.

6997-Merremia angustifolia, Hallier f. (Ipomøa angustifolia, Jacq.)

Fl. Trop. Afr. IV. ii. 111 ; Fl. Cap. IV. ii. 54; Cat. Afr. Pl. Welw. i. 729.

Matopos, Oct. Gibbs, 228; Victoria Falls, Allen, 285; April, Flanagan, 3170 ; Rogers, 5532; Bulawayo, Rand, 130.

M. palmata, Hallier $f$.

Fl. Trop. Afr. IV. ii. 108.

Bulawayo, Rand, 128, 129, 364 ; Rogers, 5753.

M. pinnata, Hallier $f$.

Fl. Trop. Afr. IV. ii. 113.

Mazoe, April, Eyles, 313 ; Victoria Falls, April, Flanagan, 3239.

M. pterygocaulos, Hallier f. (Ipomoea pterygocaulos, Choisy.)

Fl. Trop. Afr. IV. ii. 105 ; Cat. Afr. Pl. Welw. i. 727.

Victoria Falls, Allen, 39 ; Rogers, 5006* ; Engler ; July, Kolbe, 3185 ; Roger's, 5385.

7000-Astrochlæna malvacea, Hallier $f$.

Fl. Trop. Afr. IV. ii. 121 ; Fl. Cap. IV. ii. 69.

North of Hartley, Engler; Salisbury, Engler ; Mazoe, 43004800 ft. Nov. Eyles, 454 ; Chirinda, 3800 ft. Sep. Swyn. 300 ; Victoria, Monro, 1014, 566b.

var. epedunculata, Fendle.

Fl. Trop. Afr. IV. ii. 122.

Salisbury, Rand, 511; Matopos, Oct. Gibbs, 84; South Umtali Dist. Melsetter Road, 2000-3000 ft. Oct. Swyn.

A. Stuhlmanni, Hallier $f$.

Fl. Trop. Afr. IV. ii. 122.

Umtali, Cecil, 35.

7003-Ipomœa aquatica, Forsk. (I. reptans, Poir.)

Fl. Trop. Afr. IV. ii. 170 ; Cat. Afr. Pl. Welw. i. 738.

Bulawayo, Jan. Gardner, 86.

I. blepharophylla, Hallier $f$.

Fl. Trop. Afr. IV. ii. 141 ; Cat. Afr. Pl. Welw. i. 732.

Victoria Falls, Allen, 1114; North of Hartley, Engler.

I. bolusiana, Schinz. (I. angustisecta, Engl.)

Fl. Trop. Afr. IV. ii. 175 ; Fl. Cap. IV. ii. 49.

Matabeland, Elliott.

I. cardiosepala, Hochst. (I. calycina, Benth.)

Fl. Trop. Afr. IV. ii. 147 ; Fl. Cap. IV. ii. 61; Cat. Afr. Pl. Welw. i. 733 .

Bulawayo, Rand, 366 ; Rogers, 5752. 
Genus No

7003-i. Cecilae, N. E. Br.

Kew Bull. 1906, 166.

Umtali, Cecil, 36.

I. coscinosperma, Hochst. var. hirta, Rich.

Fl. Trop. Afr. IV. ii. 138 (type).

Bulawayo, Engler.

I. crassipes, Hook.

Fl. Cap. IV.'ii. 56 ; Cat. Afr. Pl. Welw. i. 732.

Victoria, Monro, 566 ; Rogers, 5755 ?.

var. cordifolia, Rendle.

Journ. Bot. 1908, 180.

Bulawayo, $4500 \mathrm{ft}$. Oct. Eyles, 21.

var. thunbergioides, Hallier $f$.

Fl. Cap. IV. ii. 56.

Bulawayo, March, Monro.

I. dammarana, Rendle.

Fl. Trop. Afr. IV. ii. 183.

Bulawayo, Rand, 273.

I. dissecta, Willd.

Fl. Cap. IV. ii. 67 ; Fl. Trop. Afr. IV. ii. 176.

Victoria Falls, Rogers, 7097*, 7129*.

I. eriocarpa, $R . B r$.

Fl. Trop. Afr. IV. ii. 136 ; Cat. Afr. Pl. Welw. i. 732.

Victoria Falls, April, Flanagan, 3301; Rogers, 5031; Bulawayo, Monro, 83.

I. fragilis, Choisy.

Fl. Trop. Afr. IV. ii. 165.

Bulawayo, Monro, 60.

var. pubescens, Hallier $f$.

Fl. Trop. Afr. IV. ii. 165.

Bulawayo, Rand, 603; Chubb, 335; Salisbury, Cecil, 75;

Matabeleland, Oates; Victoria Falls, Rogers, 7009*.

var. Randii ?.

Bulawayo, Monro, 58.

I. Holubii, Baker.

Fl. Trop. Afr. IV. ii. 188.

Matopos, Rogers, 5647.

I. involucrata, Beauv.

Fl. Trop. Afr. IV. ii. 150 ; Cat. Afr. Pl. Welw. i. 735.

Salisbury, Rand, 561.

I. lapathifolia, Hallier $f$.

Fl. Trop. Afr. IV. ii. 168.

Victoria Falls, Rogers, 5007. 
Fl. Trop. Afr. IV. ii. 187 ; Cat. Afr. Pl. Welw. i. 741.

Victoria Falls, 3000 ft. May, Eyles, 140 ; Rogers, 5145.

I. Lugardi, N. E. Br. var. parviflora, Rendle.

Fl. Trop. Afr. IV. ii. 163.

Matabeleland, Elliott.

I. magnusiana, Schinz.

Fl. Trop. Afr. IV. ii. 162 ; Fl. Cap. IV. ii. 65.

Bulawayo, Rogers, 5748 ; Victoria Falls, Rogers, 5668*.

I. obscura, Ker.

Fl. Trop. Afr. IV. ii. 164 ; Fl. Cap. IV. ii. 62.

Bulawayo, Rand, 604 ; Chubb, 91, 334 ; Matopos, Oct. Gibbs.

248 ; Odzani River Valley, Umtali, Teague, 110.

I. Papilio, Hallier $f$.

Fl. Trop. Afr. IV. ii. 167 ; Fl. Cap. IV. ii. 63.

Bulawayo, Rand, 365 ; Rogers, 5736 ; Matopos, 4500 ft. March, Eyles, 109 ; North of Hartley, Engler.

I. Pes-tigridis, $L$.

Fl. Trop. Afr. IV. ii. 158 ; Cat. Afr. Pl. Welw. i. 735.

Victoria Falls, Allen, 278.

I. pileata, Roxb.

Fl. Cap. IV. ii. 53 ; Fl. Trop. Afr. IV. ii. 151.

Victoria Falls, Rogers, 7086*

I. pubescens, Choisy, var. pubescens, Hallier $f$.

North of Hartley;, Engler.

I. Randii, Rendle.

Fl. Trop. Afr. IV. ii. 146.

Bulawayo, Rand, 271 ; Salisbury, Rand, 1423.

I. rhodesiana, Rendle.

Fl. Trop. Afr. IV. ii. 188.

Bulawayo, Rand, 141.

I. shirambensis, Baker.

Fl. Trop. Afr. IV. ii. 186.

Victoria Falls, Rogers, 5293 ; Wankie, Rogers, 5995.*

I. shupangensis, Baker.

Fl. Trop. Afr. IV. ii. 170.

South of Zambesi, Engler.

I. simplex, var. obtusisepala, Rendle.

Fl. Trop. Afr. IV. ii. 174.

Salisbury, Rand, 272, 1391 ; Bulawayo, Chubb, 397.

I. stenosiphon, Hallier $f$.

Fl. Trop. Afr. IV. ii. 192.

Matopos, 5000 ft. April, Eyles, 58 ; Victoria, Monro, 1037. 
Genus No

I. verbascoidea, Choisy. (I. Elliottii, Baker.)

Fl. Trop. Afr. IV. ii. 183 ; Cat. Afr. Pl. Welw. i. 741.

Matabeleland, Elliott; Gwai Forest, 3600 ft. Allen, 233.

I. Welwitschii, Vatke.

Fl. Trop. Afr. IV. ii. 174 ; Cat. Afr. Pl. Welw. i. 739.

Chirinda, $3500 \mathrm{ft}$. Nov. Swyn. 301.

I. Wightii, Choisy.

Fl. Trop. Afr. IV. ii. 157 ; Fl. Cap. IV. ii. 63.

Bulawayo, Rand, 555; Salisbury, Rogers, 4019; Chirinda, 3800 ft. April, Swyn. 783 ; Melsetter, 6000 ft. June, Swyn. 1165 ; Odzani River Valley, Umtali, Teague, 108.

\section{I. spp.}

Victoria Falls, Sep. Galpin, 7012; April, Flanagan, 3293; Allen, 49 ; Rogers, 5232.

Salisbury, Darling in Herb. Bolus 10784.

Bulawayo, Monro, 16.

Gwai, 2400 ft. Allen, 244.

Matopos, Rogers, 5195.

\section{FAmily CCLII.-BORAGINACEAE, Lindl.}

7038-Cordia ovalis, $R . B r$.

Fl. Trop. Afr. IV. ii. 15 ; Cat. Afr. Pl. Welw. i. 713.

Victoria, Monro, 726.

\section{C. spp.}

Matopos, Oct. Gibbs, 199.

Victoria, Monro, 767, 832, 1019.

7043-Ehretia amœna, Klotzsch. (E. mossambicensis, Klotzsch.)

Fl. Trop. Afr. IV. ii. 24 ; Fl. Cap. IV. ii. 5.

Enkeldoorn, Cecil, 89; Bulawayo, Monro, 5 ; Victoria, Monro, $730 a$; South Rhodesia, Rand, 280.

E. caffra, Sond.

Matopos, Nov. Marloth, 3381.

E. divaricata, Baker.

Fl. Trop. Afr. IV. ii. 26.

Chirinda Forest, 3700-4000 ft. Sep. Swyn. 8.

E. Fischeri, Gürke.

Fl. Trop. Afr. IV. ii. 27.

Victoria, Monro, 939.

E. hottentotica, Burch.

Fl. Cap. IV. ii. 5 ; For. Fl. Port. E. Afr. 90 ; For. Fl. Cape, 279. Bulawayo, 4500 ft. Oct. Eyles, 1069 ; Matopos, fl. \& fr. Oct. Gibbs, 279; South Rhodesia, Rand, 279. 
Genus No.

E. sp.

Bulawayo, Chubb, 344.

7051-Tournefortia sp.

Bulawayo, $4500 \mathrm{ft}$. Dec. Eyles, 20.

7052--Heliotropium marifolium, Retz.

Fl. Trop. Afr. IV. ii. 40.

Victoria Falls, Allen, 289.

H. ovalifolium, Forsk.

Fl. Trop. Afr. IV. ii. 34 ; Fl. Cap. IV. ii. 8; Cat. Afr. Pl. Welw. i. 718.

Gwai Forest, 3000 ft. Allen, 248 ; Victoria, Monro, 863.

H. zeylanicum, Lam.

Fl. Trop. Afr. IV. ii. 31; Cat. Afr. Pl. Welw. i. 720.

South Rhodesia, Rand, 106.

H. sp.

Bulawayo, Jan. Gardner, 19.

7056-Trichodesma angustifolium, Harv.

Fl. Cap. IV. ii. 11.

South Rhodesia, Rand, 636.

T. physaloides, A. DC. (Borraginoides physaloides, Hiern.)

Fl. Trop. Afr. IV. ii. 46 ; Fl. Cap. IV. ii. 11; Cat. Afr. Pl. Welw. i. 721.

Matabeleland, Oates; Elliott; Mashonaland, Bryce; Cecil ; Bulawayo, 4500 ft. Sep. Eyles, 1081; Matopos, fl. Sep. fr. Oct. Gibbs, 74; Galpin, 6994 ; Engler; Salisbury, Engler; Darling in Herb. Bolus, 10785; Rand, 388, 571; Rogers, 4018; Marandellas, Engler; Victoria Falls, Roger's, 5003 ; Umvumvumvu Riv. Oct. Swyn. 2112; Nyahodi Valley, 4000 ft. Swyn. 6003 ; Victoria, Monro, 453, 482, 530, 1023 ; South Rhodesia, Rand, 633, 634, 635; Odzani River Valley, Umtali, Teague, 267.

T. zeylanicum, R. Br. (Borraginoides zeylanica, Hiern.)

Fl. Trop. Afr. IV. ii. 51 ; Cat. Afr. Pl. Welw. i. 720.

Victoria Falls, Rogers, 7216.

7064-Cynoglossum lanceolatum, Forsk. (C. micranthum, Desf.)

Fl. Trop. Afr. IV. ii. 54; Fl. Cap. IV. ii. 14; Cat. Afr. Pl. Welw. i. 721.

Mazoe, 4400-4800 ft. Dec. Eyles, 214; Odzani River Valley, Umtali, Teague, 114.

7109 -Lithospermum sp.

South Rhodesia, Rand, 107. 


\section{Family CCLIII.-VERBENACEAE, Juss.}

Genus No

7138-Yerbena officinalis, $L$.

Fl. Trop. Afr. v. $2 \diamond 6$; Fl. Cap. V. i. 209.

Mazoe, 4800 ft. March, Eyles, 297 ; Salisbury, April, Flanagan, 3261 ; South Rhodesia, Rand, 105.

7144-Lantana salvifolia, Jacq.

Fl. Trop. Afr. v. 276 ; Fl. Cap. V. i. 190 ; Cat. Afr. Pl. Welw. i. 827.

Bulawayo, 4500 ft. Eyles, 89; Chubb, 316, 367, 380 ; Dec. Gardner, 66 ; Rogers, 5914 ; Mazoe, 4400-4800 ft. Jan. Eyles, 505, 532; Matabeleland, Marloth; Elliott; Umtali, Engler; Odzani River Valley, Teague, 3 ; Victoria Falls, Rogers, 5795 ; Salisbury, Rogers, 5795, 4069; Melsetter, 6000 ft. April, Swyn.; Chirinda, 3700-4000 ft. Dec. Swyn. 259, 116, 2002 ; Victoria, Monro, 960 ; South Rhodesia, Rand, 114.

\section{L. sp.}

Salisbury, Rand, 513.

\section{5-Lippia asperifolia, Rich}

Fl. Trop. Afr. v. 280 ; Fl. Cap. V. i. 195.

Matabeleland, Oates; Elliott; Mazoe, 4400 ft. March, Eyles, 278; Matopos, July, Galpin, 6954; Victoria Falls, Rogers; Chirinda, 3800 ft. Melsetter, 6000 ft. April, May, Dec. Swyn. 260, 475, 475a, 1160, 2003; Victoria, Monro, 311; Odzani River Valley, Umtali, Teague, 229.

L. Oatesii, Rolfe.

Fl. Trop. Afr. v. 279.

Matabeleland, Oates; Nyamandhlovu, April, Flanagan, 3194 (forma); Bulawayo, Oct. Dec. Chubb, 382 ; Rogers, 5927 ; South Rhodesia, Rand, 103.

L. Wilmsii, Pearson.

Fl. Cap. V. i. 196.

Inyanga Mts. 6000-7000 ft. Cecil, 219; Gwelo, Jan. Gardner, 47 ; Chirinda, May, Dec. Swyn. 258, 474.

\section{8-Bouchea hederacea, Sond.}

Fl. Cap. V. i. 200.

Victoria, Monro, 169, 671 ; Bulawayo, Rogers, 57 jo.

B. pinnatifida, Schauer.

Fl. Cap. V. i. 205.

Bulawayo, 4500 ft. Dec. Eyles, 1142 ; Oct. Dec. Chubb, 373 p.p. Rogers, 5919 ; between Plumtree and Bulawayo, April, Flanagan, 3210 ; South Rhodesia, Rand, 283. 
Genus No.

B. Wilmsii, Gürke.

Fl. Cap. V. i. 200.

Bulawayo, Monro, 102.

7153-Priva leptostachya, Juss.

Fl. Trop. Afr. v. 285 ; Fl. Cap. V. i. 206.

Chirinda, 3800 ft. May, June, Swyn. 269, 517.

P. sp.

Bulawayo, Eyles, 16.

7162 -Duranta Plumieri, Jacq.

Fl. Trop. Afr. v. 287 ; Fl. Cap. V. i. 210 ; Harv. Gen. S.A. Pl. 291.

Mazoe, 4200-4500 ft. Nov. Eyles, 193; Dec. Eyles, 213 ; Govt. Herb. 2083 ; Matopos, fl. \& fr. Oct. Gibbs, 280 ; Victoria, Monro, 683, 793, 1033.

Note.-According to the Floras this is an introduction from Tropical America, but it is so common in kloofs and riverbeds remote from European occupation, that this seems doubtful.

7186-Yitex Cienkowskii, Kot. \& Peyr.

Fl. Trop. Afr. v. 328 ; Cat. Afr. Pl. Welw. i. 836.

Lusitu Riv. Swyn.

V. Eylesii, S. Moore.

Journ. Bot. 1907, 154.

Bulawayo, 4500 ft. Dec. Eyles, 1201; Chirinda, 3500 ft., Chikore Hills, $3500 \mathrm{ft}$. throughout S. Melsetter Dist. fl. April, Nov. Dec. fr. May, June, Swyn. 34, 34b, 1059.

V. flavescens, Rolfe, var. parviflora, Gibbs.

Journ. Linn. Soc. Bot. xxxvii. 463; Fl. Trop. Afr. v. 321 (type).

Victoria Falls, Sep. Gibbs, 135.

Y. gürkeana, Engl.

Umtali, Engler.

Y. isotjensis, Gibbs.

Journ. Linn. Soc. Bot. xxxvii. 463.

Matopos, Isotje Hill, Oct. Gibbs, 236 ; Victoria, Monro, 803.

Y. Kirkii, Baker.

Fl. Trop. Afr. v. 321 ; For. Fl. Port. E. Afr. 94.

Victoria Falls, Allen, 60 ; Rogers, 5377.

Y. sp.

Salisbury, Rand, 553.

7191-Clerodendron amplifolium, S. Moore.

Journ. Linn. Soc. Bot. xl. 167.

Chirinda Forest, 3700-4000 ft. April, Swyn. 335. 
7191 - C. glabrum, E. Mey. (C. ovale, Klotzsch.) (Siphonanthus glabra, Hiern.)

Fl. Trop. Afr. v. 297 \& C. ovale, p. 298 partly; Fl. Cap. V. i. 219 ; Cat. Afr. Pl. Welw. i. 842; For. Fl. Port. E. Afr. 91 \& 92 ; For. Fl. Cape, 286 ; Nat. Pl. 45.

Bulawayo, Monro, 115; Matopos, Engler; Victoria Falls, Rogers, 5561; Allen, 257 (var.).

C. hirsutum, Pearson.

Fl. Cap. V. i. 221.

Chimanimani Mts. 7000 ft. Sep. Swyn. 2004; Mt. Pene, $7000 \mathrm{ft}$. Oct. Swyn. 6086.

C. lanceolatum, Giirke.

Fl. Trop. Afr. v. 312 ; For. Fl. Port. E. Afr. 93.

Victoria Falls, Allen, 99; Mazoe, 4400-4800 ft. Nov. Eyles, 447 ; Bulawayo, Rogers, 5914, 5378, 5530.

C. myricoides, $R . B r$. (Siphonanthus myricoides, Hiern.)

Fl. Trop. Afr. v. 310 \& 519 ; Fl. Cap. V. i. 223 ; For. Fl. Port. E. Afr. 93 ; Cat. Afr. Pl. Welw. i. 844 ; Nat. Pl. 282.

Matopos, Oct. Gibbs, 282 ; Bulawayo, Oct. Dec. Chubb, 383, 393, 394, 395; Chirinda, 3500 ft. Sep. Dec. Swyn. 173; Mt. Pene, 7000 ft. Swyn. 1296; Haroni Riv. 5000 ft. Swyn. 1297 ; Victoria, Monro, 734.

var. cuneatum, Pearson.

Fl. Cap. V. i. 223.

Bulawayo, Monro, 114.

var. discolor, Baker.

Fl. Trop. Afr. v. 310.

Victoria Falls, Allen, 102 ; Mazoe, 4800 ft. Nov. Eyles, 458.

C. reflexum, Pearson.

Trans. S.A. Phil. Soc. xv. 182.

Bulawayo, 4500 ft. Dec. Eyles, 1006 ; Victoria Falls, Rogers, 5634.

C. spinescens, Gürke.

Fl. Trop. Afr. v. 313 ; Fl. Cap. V. i. 221.

Victoria Falls, Allen, 402 ; Roger's, 5329.

C. Swynnertonii, S. Moore.

Journ. Linn. Soc. Bot. xl. 166.

Chirinda Forest, 3700-4000 ft. fl. \& fr. May, Swyn. 85.

C. sp.

Victoria Falls, Rogers, 5378. 
FAnily CCLIV.-LABIATAE, B. Juss.

Genus No.

7213-Tinnea rhodesiana, S. Moore.

Journ. Bot. 1905, 51.

Matopos, 4500 ft. Jan. Eyles, 159.

T. zambesiaca, Baker.

Fl. Trop. Afr. v. 499.

Bulawayo, 4500 ft. March, Eyles, 1063; Victoria, Monro, 747 or 947 .

7234-Scutellaria Livingstonei, Baker.

Fl. Trop. Afr. v. 462.

Mashonaland, Bryce; Salisbury, Sep. Rand, 520; Darling in Herb. Bolus 11198; North of Hartley, Engler; Mazoe, 4300-4800 ft. Sep. Eyles, 427, 433; Victoria, Monro.

\section{6-Acrotome sp.}

Redbank, April, Flanagan, 3188.

7264-Leonotis Melleri, Baker.

Fl. Trop. Afr. v. 491.

Odzani River Valley, Umtali, Teague, 268.

L. mollissima, Gürke.

Fl. Trop. Afr. v. 493.

Chirinda, 3700-4000 ft. June, Swyn. 235; plentiful throughout Melsetter Dist.

L. nepetifolia, $R . B r$.

Fl. Trop. Afr. v. 491 ; Cat. Afr. Pl. Welw. i. 879.

Mazoe, 5000 ft. Nov. Eyles, 462 ; Victoria, Monro, 1063.

L. randii, S. Moore.

Journ. Bot. 1900, 465.

Bulawayo, Dec. Rand, 165.

L. spectabilis, S. Moore.

Journ. Linn. Soc. Bot. xl. 180.

Chimanimani Mts. Sep. Swyn. 2013.

7268-Leucas capensis, Engl. (Lasiocorys capensis, Benth.)

Fl. Cap. V. i. 373.

Bulawayo, Dec. Rand, 162.

L. martinicensis, $R$. $B r$.

Fl. Trop. Afr. v. 479 ; Fl. Cap. V. i. 371; Cat. Afr. Pl. Welw. i. 876.

Bulawayo, May, Rand, 385 ; Mazoe, 4800 ft. March, Eyles, 294;

Marandellas, Rogers, 4042. 
Genus No.

L. milanjiana: Gürke. Fl. Trop. Afr. v. 478.

Mazoe, 4300-4800 ft. Sep. Eyles, 429; Gwelo, Jan. Gardner, 22 ; Umtali, Engler, Odzani River Valley, Teague, 185 ; Chirinda, $3800 \mathrm{ft}$. April, Swyn. 385.

L. neuflizeana, Courb.

Fl. Trop. Afr. v. 480 ; Fl. Cap. V. i. 372.

Matopos, March, Flanagan, 2960.

L. nyassae, Gïrke.

Fl. Trop. Afr. v. 485.

Salisbury, May, Flanagan, 3250 ; Bulawayo, Rogers, 5817*.

L. Randii, S. Moore.

Journ. Bot. 1900, 464.

Salisbury, July, Rand, 522.

L. stricta, Baker.

Fl. Trop. Afr. v. 484.

Victoria Falls, April, Flanagan, 3152.

L. spp.

South Rhodesia, Rand, 376; Flanagan, 3151.

7281-Stachys æthiopica, $L$.

Fl. Trop. Afr. v. 467 ; Fl. Cap. V. i. 348.

Chimanimani Mts. 7000 ft. Sep. Swyn. 1972.

7283-Achyrospermum Carvalhi, Giirke.

Fl. Trop. Afr. v. 464.

Chirinda Forest, 3700-4000 ft. May, Swyn. 78.

7290-Salvia runcinata, L. f.

El. Cap. V. i. 327.

Bulawayo, Jan. Rand, 134.

S. stenophylla, Burch.

Fl. Cap. V. i. 326.

Bulawayo, 4500 ft. Dec. Eyles, 1003.

S. sp.

South Rhodesia, Rand, 142.

7305-Satureia biflora, Briq. (Micromeria biflora, Benth.)

Fl. Trop. Afr. v. 452 ; Fl. Cap. V. i. 306.

Melsetter, 6000 ft. Sep. Swyn. 1971; Odzani River Valley, Umtali, Teague, 78.

S. masukensis (S. Moore), Torre \& Harms. (Calamintha masukensis, S. Moore.) (Leucas masukensis, Baker.)

Fl. Trop. Afr. v. 476.

Melsetter, 6000 ft. April, Swyn. 1990, 1991. 
A Record of Plants Collected in Southern Rhodesia.

Genus No

7328-Mentha longifolia, Huds. (M. sylvestris, L.)

Fl. Trop. Afr. v. 451; Fl. Cap. V. i. 303.

Salisbury, Dec. Rand, 145; Gwelo, Jan. Gardner, 1; Sr. Phil. . 33 ; Mazoe, 4400 ft. Feb. Eyles, 528.

7342-Hyptis pectinata, Poit. (Mesospherum pectinatum, O. Kuntze.)

Fl. Trop. Afr. v. 448; Fl. Cap. V. i. 297 ; Cat. Afr. Pl. Welw. i. 873 .

Victoria Falls, April, Flanagan, 3277 ; Rogers, 5025 ; Victoria, Monro, 1054 ; Odzani River Valley, Umtali, Teague, 270.

7345-Eolanthus crenatus, S. Moore.

Journ. Bot. 1907, 94.

Matopos, 5000 ft. March, Eyles, 1013 ; Victoria, Monro, 749.

\&. Rehmannii, Gürke.

Odzani River Valley, Umtali, Teague, 202.

Æ. sericeus, Gürke.

Matopos, 4800 ft. April, Eyles, 42.

E. sp.

Matopos, March, Flanagan, 2973.

7337-Pycnostachys remotifolia, Baker.

Fl. Trop. Afr. v. 381.

Mazoe, 4300 ft. April, Eyles, 325 ; Salisbury, May, Flanagan, 3273.

P. reticulata, Benth.

Fl. Trop. Afr. v. 382 ; Fl. Cap. V. i. 291.

Upper Buzi, 3500 ft. May, Swyn. 1954.

P. urticifolia, Hook.

Fl. Trop. Afr. v. 386; Fl. Cap. V. i. 291.

Salisbury, July, Rand, 525; May, Flanagan, 3274 ; Darling, in Herb. Bolus, 10786 ; Chirinda, 3800 ft. May, Swyn. 521 ; Victoria, Monro, 354, 1052.

7350 - Plectranthus caudatus, S. Moore.

Journ. Linn. Soc. Bot. xl. 176.

Chimanimani Mts. 7000 ft. Sep. Swyn. 2010.

P. chimanimanensis, S. Moore.

Journ. Linn. Soc. Bot. xl. 174.

Chimanimani Mts. 7000 ft. Sep. Swyn. 2019.

P. esculentus, $N, E$. Br.

Fl. Cap. V. i. 285.

Melsetter, Govt. Herb. 753 (cultivated).

P. floribundus, N. E. Br.

Fl. Cap. V. i. 273.

Salisbury, Aug. Rand, 524; Matopos, Sep. Gibbs, 9 : Umtali, Engler. 
Genus No.

var. longipes, $N . E . B r$.

Fl. Trop. Afr. v. 403.

Umzingwane Riv. Baines; Mazoe, 4800 ft. Aug. Eyles, 196 ; Chirinda, 3800 ft. Sep. Swyn. 237.

P. grandidentatus, Gürke.

Fl. Cap. V. i. 278.

Odzani River Valley, Umtali, Teague, 148.

P. hoslundioides, Baker.

Fl. Trop. Afr. v. 418.

Chirinda, April, Swyn. 445; Chipetzana Riv. 3000 ft. May, Swyn. 1989.

P. laxiflorus, Benth.

Fl. Cap. V. i. 276.

Chirinda Forest, April, May, Swyn. 341, 467; Odzani River Valley, Umtali, Teague, 89.

P. marrubioides, Hochst.

Fl. Trop. Afr. v. 414.

Odzani River Valley, Umtali, Teague, 127.

P. matabelensis, Baker.

Fl. Trop. Afr. v. 417.

Shashi Riv. Holub, 1403-1406.

P. petrensis, S. Moore.

Journ. Linn. Soc. Bot. xl. 175.

Chimanimani Mts. 7000 ft. Sep. Swyn. 2018.

P. selukwensis, N.E. Br.

Kew Bull. 1906, 167.

Selukwe, Cecil, 123; Mazoe, 5200 ft. Dec. Eyles, 200 ; 5000 ft.

March, Eyles, 251.

P. Swynnertonii, S. Moore.

Journ. Linn. Soc. Bot. xl. 176.

Chirinda Forest, 3700-4000 ft. fl. \& fr. Feb. Swyn. 337.

P. spp.

Victoria Falls, April, Flanagan, 3237, cf. P. Whytei, Baker.

Mazoe, March, Flanagan, 3011.

Selukwe, Jan. Gardner, 53.

Matopos, Rogers, 5254, cf. P. myrianthus, Briq.

7353-Englerastrum Schweinfurthii, Briq.

Fl. Trop. Afr. v. 445 ; Cat. Afr. Pl. Welw. i. 860.

Victoria Falls, 3000 ft. May, Eyles, 111; Allen, 410; Matopos, Rogers, 5160 (var).

7355-Coleus gazensis, S. Moore.

Journ. Linn. Soc. Bot. xl. 178.

Melsetter, $6000 \mathrm{ft}$. April, Swyn. 1998. 
C. latifolius, Hochst. Fl. Trop. Afr. v. 437.

Mazoe, 4700 ft. March, Eyles, 267.

C. matopensis, S. Moore. Journ. Bot. 1907, 96.

Matopos, $5000 \mathrm{ft}$. March, Eyles, 1024.

C. palliolatus, S. Moore. Journ. Bot. 1900, 464. Bulawayo, Jan. Rand, 144.

C. Pentheri, Gürte. Fl. Cap. V. i. 289.

Bulawayo, Rogers, 5933.

C. polyanthus, S. Moore. Journ. Bot. 1907, 96.

Matopos, 5000 ft. March, Eyles, 1012 ; Mazoe, 5300 ft. April, Eyles, 335.

C. shirensis, Gürke.

Fl. Trop. Afr. v. 443.

Umtali, Engler.

C. Swynnertonii, S. Moore. Journ. Linn. Soc. Bot. xl. 177.

Chirinda, 3700--4000 ft. May, Swyn. 349.

C. umbrosus, Vatke. Fl. Trop. Afr. v. 434.

Matopos, $4800 \mathrm{ft}$. April, Eyles, 54.

7357-Hoslundia decumbens, Benth. (H.opposita, Vahl, var. decumbens, Baker.)

Fl. Trop. Afr. v. 377 ; Fl. Cap. V. i. 298.

Victoria Falls, Rogers, 5420, 5577.

H. verticillata, Vahl.

Cat. Afr. Pl. Welw. i. 860.

Bulawayo, Dec. Rand, 163; Umtali, Engler; Chirinda, Nov. Swyn. 386.

7362 - Acrocephalus callianthus, Briq.

Fl. Trop. Afr. v. 360.

Lusitu Riv. 5000 ft. April, Swyn. 1955.

A. chirindensis, S. Moore.

Journ. Linn. Soc. Bot. xl. 181.

Chirindia, April, Swyn. 313.

A. picturatus, S. Moore.

Journ. Linn. Soc. Bot. xl. 171.

Chirinda, Swyn. 257. 
Genus No.

A. sericeus, Briq.

Fl. Trop. Afr. v. 362 ; Cat. Afr. Pl. Welw. i. 887.

Bulawayo, Jan. Rand, 146 ; Matopos, 4600 ft. April, Eyles, 46 ; Victoria, Monro, 357.

\section{A. spp.}

Mazoe, 4300 ft. April, Eyles, 324, ef. A. sericeus, Briq.

Between Plumtree and Bulawayo, April, Flanagan, 3179.

Salisbury, April, Flanagan, 3264.

7363-Geniosporum angolense, Briq.

Fl. Trop. Afr. v. 351; Fl. Cap. V. i. 293 ; Cat. Afr. Pl. Welw. i. 852.

Salisbury, March, Flanagan, 3014; April, Flanagan, 3263.

7364-Moschosma multiflorum, Benth.

Fl. Trop. Afr. v. 354.

Salisbury, May, Flanagan, 3259 ?; Odzani River Valley, Umtali, Teague, 143.

M. riparium, Hochst. (Iboza riparia, N. E. Br.; Basilicum myriostachyum, O. Kuntze.)

Fl. Trop. Afr. v. 354 \& 523 ; Fl. Cap. V. i. 300 ; Nat. Pl. 1 \& 2 : Cat. Afr. Pl. Welw. i. 858; Harv. Gen. S.A. Pl. 303.

Matopos, $\delta$ \& Sep. Gibbs, 3 (forma); Salisbury, July, Rand, 526; Mazoe, 4300 ft. o March, Eyles, 290; Bulawayo, 4600 ft. Aug. Eyles, 1234.

M. sp.

Victoria Falls, Rogers, 5133.

7365-Hemizygia flabellifolia, S. Moore.

Journ. Linn. Soc. Bot. xl. 173.

Chimanimani Mts. 7000 ft. Sep. Swyn. 1414.

H. ornata, S. Moore.

Journ. Linn. Soc. Bot. xl. 172.

Mt. Pene, 6500-7000 ft. Sep. Oct. Swyn. 1999, 6078.

7366-Ocimum americanum, L. (O. canum, Sims.)

Fl. Trop. Afr. v. 337 \& 521; Fl. Cap. V. i. 235 ; Cat. Afr. Pl. Welw. i. 848.

Bulawayo, 4500 ft. Nov. Eyles, 1219 ; Jan. Rand, 164; June, Rand, 516.

0. angustifolium, Benth. (Becium angustifolium, N. E. Br.)

Fl. Cap. V. i. 231.

Bulawayo, Rogers, 5481*.

0. Buchananii, Baker.

Fl. Trop. Afr. v. 348.

Salisbury, Rogers, 4010. 
0. filamentosum, Forsk.

Fl. Trop. Afr, v. 343.

North of Hartley, Engler.

0. knyanum, Vatke.

Fl. Trop. Afr. v. 346 ; Cat. Afr. Pl. Welw. i. 850.

Bulawayo, Oct.-Dec. Chubb, 305; Chirinda, Feb. May, Nov. Swyn. 277, 468, 1988.

0. obovatum, E. Mey. (Becium obovatum, N. E. Br.)

Nat. Pl. 257 ; Fl. Cap. V. i. 230.

Matopos, Oct. Gibbs, 102 ; Salisbury, Sep. Rand, 621 ; Darling, in Herb. Bolus; Rogers, 4009 ; Odzani River Valley, Umtali, Teague, 137.

0. odontosepalum, S. Moore.

Journ. Linn. Soc. Bot. xl. 170.

Melsetter, 6000 ft. Oct. Swyn. 6087, 6088.

0. Randii, S. Moore.

Journ. Bot. 1900, 463.

Salisbury, Sep. Rand, 618.

0. scoparium, Gürke.

Fl. Trop. Afr. v. 339.

Salisbury, May, Flanagan, 3267.

0. suave, Willd.

Fl. Trop. Afr. v. 338 ; Fl. Cap. V. i. 234 ; Cat. Afr. Pl. Welw. i. 849 ; Nat. Pl. 325.

Chirinda, 3700-4000 ft. Aug. Swyn. 384, 466.

0. sp.

Allen, Victoria Falls, 195.

7367-Orthosiphon bracteosus, Baker. (Ocimum bracteosum, Benth.; Hemizygia bracteosa, Briq.)

Fl. Trop. Afr. v. 375 ; Fl. Cap. V. i. 248.

Mazoe, 4400 ft. Dec. Eyles, 489 ; Gwai, April, Flanagan, 3295 ;

Umtali, Engler; Odzani River Valley, Teague, 21 ; Salisbury, Dec. Rand, 166 ; Rogers, 4071 ; Victoria, Monro, 973 ; Chikore Hills, 3500 ft. March, Swyn. 268 ; Umvumvumvu Riv. 4000 ft. April, Swyn. 1956; Victoria Falls, Rogers, 5608; Bulawayo, Rogers, 5482.

0. Elliottii, Baker.

Fl. Trop. Afr. v. 376 ; Fl. Cap. V. i. 249.

Bulawayo, Dec. Rand, 167; Dec. Gardner 96 ; Matabeleland, Elliott.

0. Kirkii, Baker.

Fl. Trop. Afr. v. 376.

Bulawayo, May, Rand, 384 ; Deka, 2400 ft. May, Eyles, 134. 
0. linearis, Benth.

Fl. Trop. Afr. v. 374.

Matabeleland, Oates; Bulawayo, May, Rand, 386.

0. rhodesianus, S. Moore.

Journ. Bot. 1905, 50 .

Deka, 2400 ft. May, Eyles, 132.

0. shirensis, Baker.

Fl. Trop. Afr. v. 368.

Bulawayo, Jan. Rand, 143 ; Jan. Gardner, 65; Sebawke, 4000 ft. Dec. Eyles, 71.

0. teucriifolius, N. E. Br. (Hemizygia tencriifolia, Briq.)

Fl. Cap. V. i. 254.

Mt. Pene, 7000 ft. Oct. Swyn. 6075.

0. Woodii, Gürke.

Mt. Pene, 6500-7000 ft. Sep. Swyn. 1974.

Family CCLVI.-SOLANACEAE, Hall.

7377-Nicandra physaloides, Gertn. (Pentagonia physalodes, Hiern.)

Fl. Cap. IV. ii. 109 ; Cat.' Afr. Pl. Welw. i. 752 ; Harv. Gen. S.A. Pl. 257.

Salisbury, Darling, in Herb. Bolus 10795; Odzani River Valley, Umtali, Teague, 104.

7379-Lycium tetrandrum, Thunb. (L. horridum, Thunb.)

Fl. Cap. IV. ii. 114.

Salisbury, Rand, 514; South Rhodesia, Rand, 382.

L. persicum, Miers. (L. arabicum, Schweinf.)

Fl. Trop. Afr. IV. ii. 254.

Bulawayo, $4500 \mathrm{ft}$. Oct. Eyles, 1073; Monro, 10; Salisbury, Rand, 515; South Rhodesia, Rand, 147.

7400-Withania somifera, Dunal. (Physaloides somnifera, Moench.)

Fl. Trop. Afr. IV. ii. 249 ; Fl. Cap. IV. ii. 107 ; Cat. Afr. Pl. Welw. i. 752 ; Harv. Gen. S.A. Pl. 258.

Bulawayo, 4500 ft. Dec. Eyles, 15; Tati Riv. Holub; Mazoe, 4300 ft. Jan. Eyles, 239; Rusapi, Engler; Matabeleland, Marloth.

7401-Physalis minima, L.

Fl. Trop. Afr. IV. ii. 247 ; Fl. Cap. IV. ii. 106; Cat. Afr. Pl. Welw. i. 750.

Victoria Falls, April, Flanagan, 3305. 
Genus No.

P. peruviana, $L$.

Fl. Trop. Afr. IV. ii. 248 ; Fl. Cap. IV. ii. 106 ; Harv. Gen. S.A. Pl. 257.

Mazoe, 4800 ft. Jan. Eyles, 236 ; Odzani River Valley, Umtali, Teague, 129.

7407-Solanum acanthocalyx, Klotzsch.

Fl. Trop. Afr. IV. ii. 234.

Chirinda Forest, 3700-4000 ft. Nov. Swyn. 93.

S. aculeastrum, Dun.

Fl. Trop. Afr. IV. ii. 243 ; Fl. Cap. IV. ii. 95 ; For. Fl. Cape, 278.

Chirinda, cultivated for hedges, Swyn.

S. bifurcum, Hochst.

Fl. Trop. Afr. IV. ii. 213 ; Fl. Cap. IV. ii. 94 ; Cat. Afr. Pl. Welw. i. 746.

Chirinda Forest, 3700-4000 ft. Oct. Swyn. 86.

S. incanum, $L$.

Fl. Trop. Afr. IV. ii. 238; Fl. Cap. IV. ii. 101.

Salisbury, Rogers, 5802.

S. indicum, $L$.

Fl. Trop. Afr. IV. ii. 232 ; Fl. Cap. IV. ii. 101 ; Cat. Afr. Pl. Welw. i. 750.

Chirinda, 3800 ft. fl. Oct. fr. May, Swyn. 385, 482, on cultivated ground.

S. nigrum, $L$.

Fl. Trop. Afr. IV. ii. 218; Fl. Cap. IV. ii. 89 ; (at. Afr. Pl. Welw. i. 745.

Bulawayo, 4500 ft. Jan. Eyles, 1009; Rogers, 6202 ; Victoria Falls, Rogers, 5131; Victoria, Monro, 1024 ; South Rhodesia, Rand, 171; Odzani River Valley, Umtali, Teague, 74.

S. nodiflorum, Jacq.

Fl. Trop. Afr. IV. ii. 218 ; Cat. Afr. Pl. Welw. i. 745.

Melsetter, 6000 ft. fl. \& fr. April, May, Swyn. 342; Chirinda Forest, 3800 ft. Swyn. 481; Chirinda, on cultivated ground, Swyn. 1794.

S. panduræforme, E. Mey. (S. delagoense, Dun.)

Fl. Trop. Afr. IV. ii. 214 ; Fl. Cap. IV. ii. 99; Cat. Afr. Pl. Welw. i. 747 ; Nat. Pl. 596.

Matabeleland, Oates; Elliott; Sebakwe, 4000 ft. Dec. Eyles, 123 ; Plumtree, April, Flanagan, 3177 ; Bulawayo, Chubb, 390 ; Rogers, 5504 ; Chirinda, 3800 ft. Nov. Swyn. 1793 ; Salisbury, Rogers, 5772 ; Matopos, May, Rogers; Victoria Falls, April, Rogers; South Rhodesia, Rand, 170. 
S. sodomeum, var. Hermanni, Dun.

Fl. Cap. IV. ii. 96.

Bembesi, Jan. Gardner, 49.

7415-Datura Stramonium, $L$.

Fl. Trop. Afr. IV. ii. 257 ; Fl. Cap. IV. ii. 118; Cat. Afr. Pl.

Welw. i. 753 ; Harv. Gen. S.A. Pl. 258.

Mazoe, 4400 ft. March, Eyles, 280 ; Bulawayo, Chubb, 349 ;

South Rhodesia, Rand, 169.

7436-Petunia violacea, Lindl.

Umgusa Riv. 4400 ft. Nov. Eyles, 1189.

FAmily CCLVII.-SCROPHULARIACEAE, Lindl.

7467-Apostimum decumbens, Schinz.

Fl. Trop. Afr. IV. ii. 272 ; Cat. Afr. Pl. Welw. i. 755.

Victoria Falls, Allen, 105; April, Flanagan, 3202 ; Rogers, 7212.

A. elongatum, Engl.

Fl. Trop. Afr. IV. ii. 273.

Mashonaland, Bryce; South Rhodesia, Rand, 641.

A. lineare, Marl. \& Engl. (A. Randii, S. Moore.)

Fl. Trop. Afr. IV. ii. 269 ; Fl. Cap. IV. ii. 129 ; Cat. Afr. Pl. Welw. i. 755.

Bulawayo, 4500 ft. Nov. Eyles, 87 ; Cecil, 115 ; Rand, 180 ; Rogers, 5925; Jan. Gardner, 63 ; Chubb, 327 ; Matopos, Sep. Gibbs, 13; Gwelo, Sr. Phil. 57; Matabeleland, Elliott; Marloth.

7476 - Nemesia affinis, Bentin.

Fl. Trop. Afr. IV. ii. 286 ; Fl. Cap. IV. ii. 182.

Matopos, Sep. Gibbs, 49.

N. dentata, G. Don.

Fl. Trop. Afr. IV. ii. 286.

Matabeleland, Herb. Desfontaines.

N. divergens, Benth.

Fl. Cap. IV. ii. 193.

Bulawayo, 4600 ft. Aug. Eyles, 1229.

N. fœtens, Vent. Fl. Cap. IV. ii. 193.

Bulawayo, 4500 ft. Dec. Eyles, 31 ; Matopos, Sep Gibbs, 312.

7477-Diclis petiolaris, Benth.

Fl. Trop. Afr. IV. ii. 287 ; Fl. Cap. IV. ii. 201.

Bulawayo, May, Rand, 372; Matopos, Sep. Gibbs, 76 ; March, Flanagan, 2955 ; Victoria Falls, Allen, 409. 
Genus No.

7493 - Halleria lucida, $L$.

Fl. Trop. Afr. IV. ii. 295 ; Fl. Cap. IV. ii. 207 ; Nat. Pl. 545.

Chirinda Forest, 3700-4000 ft. Sep. Swyn. 124; Melsetter, $6000 \mathrm{ft} .$, Dec. Swyn. 1138.

7494-Teedia lucida, Rud.

Fl. Cap. IV. ii. 210.

Chimanimani Mts. 7000 ft. Sep. Swyn. 785.

7497-Freylinia tropica, S. Moore.

Journ. Linn. Soc. Bot. xl. 152.

Melsetter, 6000 ft. Sep. Swyn. 608.

7517-Manulea rhodesiana, S. Moore.

Journ. Bot. 1911, 156.

Victoria, Monro, 924.

7519-Sutera atropurpurea, Hiern. (Lyperia atropurpurea, Benth.)

Fl. Trop. Afr. IV. ii. 309 ; Fl. Cap. IV. ii. 306.

Bulawayo, May, Rand, 370 ; June, Eyles, 148.

S. Bolusii, Hiern.

Fl. Cap. JV. ii. 300.

Bulawayo, Chubb, $6 a$.

S. brunnea, Hiern.

Fl. Cap. IV. ii. 305.

Bulawayo, Rogers, 5749.

S. burkeana, Hiern. (Lyperia burkeana, Benth.)

Fl. Trop. Afr. IV. ii. 308; Fl. Cap. IV. ii. 299; Cat. Afr. Pl. Welw. i. 757.

Matabeleland, Baines; Oates; Bulawayo, May, Rand, 371; Chubb, 317, 331; Matopos, fl. \& fr. Sep. Oct. Gibbs, 15; March, Flanagan, 2994; Gwelo, Sr. Phil. 5; Odzani River Valley, Umtali, Teague, 18.

S. Caryalhoi, Skan.

Fl. Trop. Afr. IV. ii. 307.

Inyanga, Cecil, 198; Melsetter, 6000 ft. Sep. Swyn. 1980 ; North Melsetter, 5000-6000 ft. Oct. Swyn. 6228.

S. floribunda, O. Kuntze.

Fl. Cap. IV. ii. 277.

Melsetter, 6000 ft. Sep. Swyn. 1996; North Melsetter, 5000$6000 \mathrm{ft}$. Oct. Swyn. 6149.

S. micrantha, Hiern. (S. fissifolia, S. Moore; Chaenostoma micranthum, Engl.)

Fl. Trop. Afr. IV. ii. 303 ; Fl. Cap. IV. ii. 263; Journ. Bot. 1900, 467.

Bulawayo, Jan. Rand, 155 ; Oct. Eyles, 1077 ; Gwelo, Gardner, 16 ; Matengwe Riv. Holub, 1312, 1313 ; Matopos, Sep. Gibbs, 36; Bulawayo, 4500 ft. Aug. Eyles, 1243. 
Genus No. $\mathbf{S}$. spp.

Bulawayo, Rogers, 13612 and 5681.

7523-Zaluzianskya capensis, Walp.

Fl. Cap. IV. ii. 338.

Melsetter, 6000 ft. Sep. Swyn. 1981.

7524-Mimulus gracilis, R. Br. (M. angustifolius, Hochst.; M. strictus, Benth.)

Fl. Trop. Afr. IV. ii. 310 ; Fl. Cap. IV. ii. 354 ; Cat. Afr. Pl. Welw. i. 758.

Bulawayo, Dec. and May, Rand, 156, 368, 506 ; Dec. Eyles, 57 ; Aug. Eyles, 1230 ; Gardner, 70 ; Rogers, 13604.

7532 -Limnophila ceratophylloides, Skan. (Stemodiacra ceratophylloides, Hiern.)

Fl. Trop. Afr. IV. ii. 317 ; Cat. Afr. Pl. Welw. i. 759.

Salisbury, Rand, 539.

L. gratioloides, $R . B r$.

Fl. Trop. Afr. IV. ii. 319.

Salisbury, July, Rand, 518.

L. sessiflora, Blume.

Fl. Trop. Afr. IV. ii. 318.

Matopos, Oct. Gibbs, 212.

L. sp.

Mazoe, 4300 ft. Jan. Eyles, 511.

7535-Stemodiopsis Eylesii, S. Moore.

Journ. Bot. 1908, 71.

Mazoe, $5000 \mathrm{ft}$. March, Eyles, 252.

S. humilis, Skan.

Fl. Trop. Afr. IV. ii. 316.

Matopos, 5000 ft. Feb. Eyles, 1166.

7558-Limosella aquatica, L. var. tenuifolia, Hook.f.

Fl. Trop. Afr. IV. ii. 352 ; Fl. Cap. IV. ii. 357.

Mazoe, 4200 ft. Sep. Eyles, 408, 409.

7560 -Craterostigma nanum, Oliv.

Fl. Trop. Afr. IV. ii. 331; Fl. Cap. IV. ii. 360.

Inyanga, 6000-7000 ft. Cecil, 208.

C. plantagineum, Hochst.

Fl. Trop. Afr. IV. ii. 329; Fl. Cap. IV. ii. 361; Cat. Afr. Pl. Welw. i. 761.

Matabeleland, Oates; Bulawayo, Dec. Rand, 157 ; Chubb, 401 ;

Iron Mine Hill, Cecil, 93 ; Victoria, Monro, 838; Matopos, Nov. Marloth, 3405.

C. $\mathrm{sp}$.

Victoria, Monro, 788. 
A Record of Plants Collected in Southern Rhodesia.

Genus No.

7564 -Ilysanthes conferta, Hiern. (I. Plantaginella, S. Moore.)

Fl. Trop. Afr. IV. ii. 347; Fl. Cap. IV. ii. 365; Journ. Bot. $1905,49$.

Matopos, 4500 ft. April; Eyles, 47; March, Flanagan, 2987.

I. micrantha, S. Moore.

Journ. Bot. 1911, 158.

Victoria, Monro, 1031.

I. Muddii, Hiern.

Fl. Cap. IV. ii. 366.

Matopos, $4500 \mathrm{ft}$. March, Eyles, 1033.

7566-Hebenstreitia dentata, L. $f$.

Fl. Cap. V. i. 101 ; Cat. Afr. Pl. Welw. i. 825.

Nyahodi Riv. 5000 ft. April, Swyn. 1888.

H. elongata, Bolus.

Fl. Cap. V. i. 99.

Mt. Pene, 6500-7000 ft. Sep. Swyn. 1887.

H. Holubii, Rolfe.

Victoria Falls, fl. \& fr. Sep. Gibbs, 116 ; Engler; Odzani River Valley, Umtali, Teague, 230.

7568-Selago Cecilae (Rolfe), Torre \& Harms. (Walafrida Cecilae, Rolfe.) Kew Bull. 1906, 167.

Bulawayo, Cecil.

S. chongweensis (Rolfe), T. \& H. (Walafrida chongweensis, Rolfe.) Journ. Linn. Soc. Bot. xxxvii. 462.

Victoria Falls, Allen, 120 ; Sep. Gibbs, 117.

S. Hoepfneri, Rolfe.

Sicumy Vlei, $3200 \mathrm{ft}$. Allen, 231.

S. Swynnertonii (S. Moore), T. \& H. (Walafrida Swynnertonii, S. Moore.)

Journ. Linn. Soc. Bot. xl. 165.

Nyahodi Riv. 5000 ft. fl. \& fr. April, Swyn. 2135.

S. spp.

Matopos, Engler, cf. S. micrantha, Choisy.

Bulawayo, Rand, 551.

7597-Melasma communis (Hemsl.), Torre \& Harms. (Alectra communis, Hemsl.)

Fl. Trop. Afr. IV. ii. 372.

Chirinda, 3700-4000 ft. June, Swyn. 1975.

M. orobanchoides, Engl. (Alectra pumila, Benth.; Alectra kilimandjarica, Hemsl.)

Fl. Trop. Afr. IV. ii. 365, where see note that this name probably inchudes several distinct species.

Fl. Cap. IV. ii. 376 ; Cat. Afr. Pl. Welw. i. 768.

Mazoe, 5000 ft. April, Eyles, 332 ; March, Eyles, 289 ; Matopos, $5000 \mathrm{ft}$. March, Eyles, 1016. 
Genus No.

M. sessiliflorum, Hiern. (Alectra melampyroides, Benth.)

Fl. Trop. Afr. IV. ii. 371; Fl. Cap. IV. ii. 375; Cat. Afr. Pl. Welw. i. 767 ; Nat. Pl. 397.

Victoria Falls, Sep. Gibbs, 293 ; Rogers, 5780.

\section{M. sp.}

Victoria Falls, April, Flanagan, 3163, cf. M. indicum, Hiern.

7614-Graderia scabra, Benth. (Bopusia scabra, Presl.)

Fl. Cap. IV. ii. 389 ; Cat. Afr. Pl. Welw. i. 772.

Mt. Pene, 6500-7000 ft. Sep. Swyn. 1977, 1978; Haroni Riv. $5500 \mathrm{ft}$. Oct. Swyn. 6077.

7616-Sopubia cana, Harv.

Fl. Cap. IV. ii. 386 ; Cat. Afr. Pl. Welw. i. 774.

Mazoe, $4300 \mathrm{ft}$. April, Eyles, 322.

S. leprosa, S. Moore.

Fl. Trop. Afr. IV. ii. 453 ; Journ. Bot. 1900, 468.

Salisbury, Dec. Rand, 158.

S. ramosa, Hochst.

Fl. Trop. Afr. IV. ii. 449 ; Cat. Afr. Pl. Welw. i. 773.

Salisbury, May, Flanagan, 3272 ; Chirinda, 3800 ft. fl. \& fr. April, June, Swyn. 389; Chipetzana Riv. 3000 ft. Swyn. 2125; Upper Buzi Riv. 3000 ft. Swyn. 2126; Odzani River Valley, Umtali, Teague, 224; Victoria Falls, Rogers, 13310*; Matopos, Rogers, $7919 *$

S. simplex, Hochst.

S. dregeana, Benth.

In Fl. Cap. IV. ii. 386, 1904, these two species are treated as synonymous, but in Fl. Trop. Afr. IV. ii. 450, 451, 1906, they are again separated. As I am unable to allocate all the records of various collectors, I give them here, distinguishing the species where known:-

See also Cat. Afr. Pl. Welw. i. 773 \& Nat. Pl. 364.

S. simplex, Matabeleland, Bryce; Matopos, Oct. Gibbs, 99 ; Mazoe, 4300 ft. Dec. Eyles, 481; Mt. Pene, 6500-7000 ft. Sep. Oct. Swyn. 1976, 6209.

S. dregeana, Umtali, Cecil, 234.

Doubtful, Matopos, 4800 ft. Nov. Eyles, 1102 ; Salisbury, Dec. Rand, 159 ; Victoria, Monro, 582, 799, 948, 1028.

\section{2-Buchnera ciliolata, Engl.}

Fl. Trop. Afr. IV. ii. 379 ; Cat. Afr. Pl. Welw. i. 775.

Victoria, Monro, 1029.

B. dura, Benth.

Fl. Cap. IV. ii. 391.

Matopos, 5000 ft. March, Eyles, 1014. 
Genus No.

B. Eylesii, S. Moore.

Journ. Bot. 1908, 72.

Mazoe, 5000 ft. April, Eyles, 334.

B. Henriquesii, Engl. (B. rhodesiana, S. Moore.)

Fl. Trop. Afr. IV. ii. 396 ; Journ. Bot. 1900, 468; Cat. Afr. Pl. Welw. i. 775.

Salisbury, Dec. Rand, 154; Engler; Matabeleland, Elliott; Mashonaland, Bryce; Charter Flats, Cecil, 83; Bulawayo, 4500 ft. Nov. Eyles, 1111; Chirinda, 3800 ft. May-Nov. Swyn. 303a; Melsetter, 6000 ft. Swyn. 1982; Upper Buzi, 3500 ft. Swyn. 6106; Mt. Pene, 7000 ft. Swyn. 6109; Victoria Falls, Rogers, 7295.

B. hispida, Buch.-Ham.

Fl. Trop. Afr. IV. ii. 397.

Victoria Falls, Rogers, 7071*, 7093*.

B. Lastii, Engl.

Fl. Trop. Afr. IV. ii. 392.

Mt. Pene, 7000 ft. Oct. Nov. Swyn. 6105, 6150.

B. leptostachya, Benth.

Fl. Trop. Afr. IV. ii. 394.

Selukwe, Rogers, 4093 ?.

B. pusilliflora, S. Moore.

Journ. Bot. 1908, 310.

Mazoe, $4300 \mathrm{ft}$. April, Eyles, 367.

B. Randii, S. Moore.

Fl. Trop. Afr. IV. ii. 387 ; Journ. Bot. 1900, 467.

Salisbury, July, Rand, 573.

B. sp.

Matopos, March, Flanagan, 2985, 2971.

7622a-Eylesia buchneroides, S. Moore.

Journ. Bot. 1908, 311.

Mazoe, 4300 ft. April, Eyles, 366.

7623-Cycnium adonense, E. Mey.

Fl. Trop. Afr. IV. ii. 431 ; Fl. Cap. IV. ii. 395 ; Cat. Afr. Pl. Welw. i. 777 ; Nat. Pl. 273.

Bulawayo, 4500 ft. Dec. Eyles, 18; Dec. Rand, 161; Salisbury, Darling; Chipete Forest, 3800 ft. April, Swyn. 234; Melsetter, 6000 ft. Oct. Swyn. 6076; also Chirinda, Lusitu Valley, etc. Odzani River Valley, Umtali, Teague, 121.

\section{C. palustre?.}

Victoria Falls, Davy. 
Genus No.

7624-Rhamphicarpa fistulosa, Benth.

Fl. Trop. Afr. IV. ii. 419; Fl. Cap. IV. ii. 398; Cat. Afr. Pl. Welw. i. 778.

Bulawayo, May, Rand, 387; Matopos, March, Flanagan, 2963a.

B. montana, N. E. Br.

Fl. Trop. Afr. IV. ii. 427; Kew Bull. 1901, 129 ; Fl. Cap. IV ii. 400 .

Matabeleland, Elliott; Bulawayo, Rand, 160 ; between Charter and Bulawayo, Cecil, 90 ; Bulawayo, 4500 ft. Nov. Eyles, 1112 ; Rogers, 5511; Chubb, 399 ; Victoria, Monro, 611, 792.

R. tubulosa, Benth. (Cycnium tubulosum, Engl.)

Fl. Trop. Afr. IV. ii. 428 ; Fl. Cap. IV. ii. 399; Harv. Gen. S.A. Pl. 271.

Victoria Falls, Sep. Galpin; Allen, 34; fl. \& fr. Sep. Gibbs, 131, 101 ; Engler; April, Flanagan, 3164; Rogers, 5277; Mashonaland, Bryce; Victoria, Monro, 921, 948a.

\section{R. sp.}

Matopos, March, Flanagan, 2963 ?, n. sp.

7625-Striga elegans, Benth.

Fl. Trop. Afr. IV. ii. 408; Fl. Cap. IV. ii. 382 ; Cat. Afr. Pl. Welw. i. 779.

Mashonaland, Cecil, 259 ; Mazoe, 4400 ft. April, Eyles, 368; Victoria, Monro, 975.

S. Forbesii, Benth.

Fl. Trop. Afr. IV. ii. 410 ; Fl. Cap. IV. ii. 384 ; Cat. Afr. Pl. Welw. i. 780.

Victoria Falls, April, Flanagan, 3155.

S. lutea, Lour. (S. coccinea, Benth.)

Fl. Trop. Afr. IV. ii. 409 ; Fl. Cap. IV. ii. 382 ; Cat. Afr. Pl. Welw. i. 780 .

Gwelo, Jan. Gardner, 42 ; Matabeleland, Cecil, 128 ; Matopos, March, Flanagan, 2992 ; Upper Buzi, 3000 ft. April, Swyn. 1985 ; Odzani River Valley, Umtali, Teague, 84.

S. orobanchoides, Benth.

Fl. Trop. Afr. IV. ii. 402 ; Fl. Cap. IV. ii. 380 ; Cat. Afr. Pl. Welw. i. 778.

Matopos, March, Flanagan, 2993 ; Mazoe, 4600 ft. Jan. Eyles, 508; Victoria Falls, April, Flanagan, 3202 ; Rogers, 7023.

\section{S. Thunbergii, Benth.}

Fl. Trop. Afr. IV. ii. 404 ; Fl. Cap. IV. ii. 380 ; Cat. Afr. Pl. Welw.i. 779 ; Nat. Pl. 370.

Salisbury, July, Rand, 521; Engler; Manica, Cecil, 159; Gwelo, Jan. Gardner, 54; Matabeleland, Elliott; Victoria, Monro, 976. 


\section{Family CCLVIII.-BIGNONIACEAE, Pers.}

Genus No.

7716 a-Podranea Brycei, Sprague. (Tecoma Brycei, N. E. Br.)

Fl. Trop. Afr. IV. ii. 515; Kew Bull. 1901, 130.

Mashonaland, Bryce; Bulawayo, Franklin White; Mazoe, 4300 ft. June, Eyles, 373 ; Victoria, Monro, 1087 ; Chipete Forest, 3800 ft. May, Swyn. 429 ; Melsetter Dist. 3000-5000 ft. Swyn.; Selukwe, Rogers, 4099.

7722-Rhigozum brevispinosum, O. Kuntze.

Fl. Trop. Afr. IV. ii. 531.

Shashi Riv. Baines.

7744-Markhamia acuminata, Sprague.

Fl. Trop. Afr. IV. ii. 524.

Sebakwe, 4000 ft. Dec. Eyles, 171 ; Victoria Falls, Allen, 149.

M. lanata, K. Schum.

Fl. Trop. Afr. IV. ii. 527.

Upper Buzi, 3000 ft. Dec. Swyn. 33; also western slopes of Mpengo Mt., Chirinda, Chikore Hills and Umswirizwi.

7761-Kigelia pinnata, DC. var. tomentella, Sprague.

Fl. Trop. Afr. IV. ii. 537; \& for type Fl. Cap. IV. ii. 454 ; Nat. Pl. 386, 387 ; Cat. Afr. Pl. Welw. i. 793 ; For. Fl. Port. E. Afr. 91; Harv. Gen. S.A. Pl. 276.

Victoria Falls, Sep. Galpin, 7025 ; Davy ; Cartwright; Allen, 30 ; Salisbury, Darling; Gwanda, Noble, 38 ; Mazoe, Govt. Herb. 929 ; Victoria, Monro, 495.

\section{Family CCLIX.-PEDALIACEAE, Lindl.}

7769-Pterodiscus Elliottii, Baker.

Fl. Trop. Afr. IV. ii. 542.

Matabeleland, Elliott.

P. speciosus, Hook.

Fl. Trop. Afr. IV. ii. 542 ; Fl. Cap. IV. ii. 456.

Shashi Riv. Rand, 197; Victoria, Monro, 659, 825.

\section{P. sp.}

Gwai Forest, Jan. Allen, 272.

7771-Harpagophytum procumbens, DC.

Fl. Trop. Afr. IV. ii. 548; Fl. Cap. IV. ii. 458.

Victoria Falls, Allen, 74; April, Flanagan, 3240; Bulawayo, Rogers, 5602.

var. sublobatum, Engl.

Fl. Trop. Afr. IV. ii. 548.

Matabeleland, Elliott; Shashi Riv. Rand, 200; Victoria Falls, Rogers, 5384. 
Genus No.

7776-Rogeria adenophylla, J. Gay.

Fl. Trop. Afr. IV. ii. 549; Cat. Afr. Pl. Welw. i. 796.

South Rhodesia, Rand, 199.

7777-Sesamum alatum, Thonn.

Fl. Trop. Afr. IV. ii. 559.

South Rhodesia, Rand, 378.

S. angustifolium, Engl.

Fl. Trop. Afr. IV. ii. 554.

Mazoe, 4800 ft. Jan. Eyles, 234; Victoria Falls, Allen, 132 ; Rogers, 5140, 5601, 7242, 7144 ; July, Kolbe, 3134; April, Flanagan, 3161.

S. Baumii, Stapf.

Fl. Trop. Afr. IV. ii. 554.

Victoria Falls, Sep. Gibbs, 115.

S. calycinum, Welw.

Fl. Trop. Afr. IV. ii. 555 ; Cat. Afr. Pl. Welw. i. 797.

Salisbury, Cecil, 142; Rand.

S. capense, Burm.f. (Sesamopteris pentaphylla, DC.)

Fl. Trop. Afr. IV. ii. 560 ; Fl. Cap. IV. ii. 461; Cat. Afr. Pl. Welw. i. 800 ; Harv. Gen. S.A. Pl. 278.

Bulawayo, Jan. Gardner, 60 ; Eyles; Matabeleland, Marloth; South Rhodesia, Rand, 196.

S. indicum, $L$.

Fl. Trop. Afr. IV. ii. 558; Fl. Cap. IV. ii. 460 ; Cat. Afr. Pl. Welw. i. 797.

Chirinda, 3800 ft. cultivated ground, April, Swyn. 251; Upper Buzi, 3000 ft. May, Swyn. 2000.

7778 - Ceratotheca kraussiana, Kunth.

Victoria, Monro, 993.

C. sesamoides, Endl.

Fl. Trop. Afr. IV. ii. 563.

Deka, 2400 ft. May, Eyles, 133 ; Victoria Falls, April, Flanagan, 3204 ; Rogers, 5621.

C. triloba, E. Mey.

Fl. Trop. Afr. IV. ii. 564 ; Fl. Cap. IV. ii. 462 ; Nat. Pl. 367.

Shashi Riv. Holub; Matengwe Riv. Holub, 1283 ; Bulawayo, Rand, 379; Matabeleland, Elliott; Rogers, 5112; between Umtali and Salisbury, Cecil, 52 ; Odzani River Valley, Umtali, Teague, 11 ; Manica, Bryce; Matopos, 4500 ft. March, Eyles, 107 ; Oct. Gibbs, 258; Rogers, 5365, 5408, 1112 ; Salisbury, May, Flanagan, 3258; North Melsetter, 5000-6000 ft. Oct. Swyn. 6221; Victoria, Monro, $1093 a$. 
Genus No.

7780-Pretrea zanguebarica, J. Gay. (P. eriocarpa, Decne.)

Fl. Trop. Afr. IV. ii. 565; Fl. Cap. IV. ii. 463; Cat. Afr.

Pl. Welw. i. 801 ; Harv. Gen. S.A. Pl. 277.

Bulawayo, $4500 \mathrm{ft}$. Nov. Eyles, 80 ; Monro, 3 \& 17 ; Salisbury, Cecil, 73; Matopos, Oct. Gibbs, 264; Engler; March, Flanagan, 2995 ; Victoria Falls, Allen, 157 ; Umtali, Engler ; Odzani River Valley, Teague, 94; Matabeleland, Marloth; South Rhodesia, Rand, 198, 377.

FAmily CCLXI.-OROBANCHACEAE, Lindl.

7791-Orobanche minor, Sutton.

Fl. Trop. Afr. IV. ii. 467.

Mazoe, 5000 ft. April, Eyles, 331.

Family CCLXII.-GESNERIACEAE, Nees.

\section{3-Streptocarpus spp.}

Matopos, 5000 ft. March, leaf only, Eyles, 1041 ; Nov. fl. Eyles, 1097 ; Selukwe, Rogers.

Family CCLXIV.-LENTIBULARIACEAE, Lindl.

7899-Genlisea africana, Oliv.

Fl. Trop. Afr. IV. ii. 497 ; Cat. Afr. Pl. Welw. i. 789.

Matopos, fl. \& fr. Oct. Gibbs, 219 ; Victoria Falls, Engler.

7901-Utricularia exoleta, $\mathrm{R} . \mathrm{Br}$.

Fl. Trop. Afr. IV. ii. 495 ; Fl. Cap. IV. ii. 435 ; Cat. Afr. Pl. Welw. i. 786.

Victoria Falls and Matopos, Oct. Gibbs, 174; Victoria Falls, Engler; July, Kolbe, 3151 ; Rogers, 5291.

U. firmula, Welw.

Fl. Trop. Afr. IV. ii. 479 ; Cat. Afr. Pl. Welw. i. 788.

Victoria Falls, Kirk; Victoria Falls and Matopos, Sep. Gibbs, 172, 173.

U. Gibbsiae, Stapf.

Fl. Trop. Afr. IV. ii. 574.

Victoria Falls, Sep. Gibbs, 177 ; Rogers, 5024; Galpin, 7038; Engler. 
Genus No

U. Kirkii, Stapf.

Fl. Trop. Afr. IV. ii. 476 ; Fl. Cap. IV. ii. 428.

Victoria Falls, Sep. Gibbs, 176; Sep. Galpin, 7039; Engler; Rogers, 5027.

U. transrugosa, Stapf.

Fl. Trop. Afr. IV. ii. 473 \& 574 ; Fl. Cap. IV. ii. 428.

Salisbury, Rand, 517 ; Matopos, Sep. Gibbs, 50 ; 4500 ft. Nov.

Eyles, 1099 partly.

U. Welwitschii, Oliv.

Fl. Trop. Afr. IV. ii. 478; Cat. Afr. Pl. Welw. i. 788.

Matopos, Oct. Gibbs, 230, 214.

U. sp.

Mazoe, 4300 ft. Jan. Eyles, 510.

Family CCLXVI.-ACANTHACEAE, Juss.

7909 - Nelsonia campestris, $R . B r$.

Fl. Trop. Afr. v. 28 ; Cat. Afr. P1. Welw. i. 805.

Victoria Falls, Sep. Galpin, 7026.

N. tomentosa, Willd.

Victoria Falls, Sep. Gibbs, 122.

7914-Thunbergia affinis, S. Moore.

Fl. Trop. Afr. v. 11; Cat. Afr. Pl. Welw. i. 801.

Mazoe, 4200-4600 ft. Dec. Eyles, 191, 199.

T. alata, $B 0_{j}$.

Fl. Trop. Afr. v. 16 ; Fl. Cap. V. i. 10 ; Nat. Pl. 300 ; Cat. Afr. Pl. Welw. i. 803.

Khami, March, Eyles, 10 ; Chirinda, 3700-4000 ft. Jan. Swyn. 353 ; Chipete Forest, 3000 ft. May, Swyn. 452.

T. Bachmanni, Lindau.

Fl. Cap. V. i. 8.

Victoria, Monro, 652, 653a.

T. glaberrima, Lindau.

North of Hartley, Engler.

T. lancifolia, T. Anders.

Fl. Trop. Afr. v. 25 ; Cat. Afr. Pl. Welw. i. 804.

Salisbury, Sep. Rand, 631, 1383; Darling, in Herb. Bolus, 11194 ;

Inyati Riv. Bryce; Mazoe, 4300-4800 ft. Oct. Nov. Eyles, 190 ; Nyahodi Riv. 5000 ft. Sep. Swyn. 1925.

T. Monroi, S. Moore.

Journ. Bot. 1913, 209.

Victoria, Monro, 652a, 653. 
Genus No.

T. oblongifolia, Oliv. var. Berringtonii, Burkill.

Fl. Trop. Afr. v. 24 \& 508.

Mashonaland, Berrington.

T. Randii, S. Moore.

Fl. Trop. Afr. v. 508 ; Journ. Bot. 1900, 201.

Bulawayo, Dec. Rand, 275, 276; Mazoe, 4400-4600 ft. Dec. Eyles, 479 ; Salisbury, Rand, 1381; Gwelo, Sr. Phil. 49 ; Umvumvumvu Riv. 4000 ft. Oct. Swyn. 6098; Mt. Pene, 7000 ft. Swyn. 6216.

T. Swynnertonii, S. Moore.

Journ. Linn. Soc. Bot. xl. 157.

Chirinda Forest, 3700-4000 ft. May, Swyn. 339.

7915-Pseudocalyx africanus, S. Moore.

Journ. Linn. Soc. Bot. xl. 156.

Chirinda Forest, 3800 ft. May, Swyn. 97.

7924-Synnema Ácinos, S. Moore.

Journ. Bot. 1908, 73.

Bulawayo, 4500, Aug. Eyles, 1242 ; Victoria, Monro, 414a, 590.

7925-Brillantaisia grandidentata, S. Moore.

Journ. Bot. 1907, 331.

Mt. Pene, 6500-7000 ft. Sep. Swyn. 336, 1145.

7926-Hygrophila cataractae, S. Moore.

Journ. Linn. Soc. Bot. xxxvii. 459.

Victoria Falls, Sep. Gibbs, 159 ; Sep. Galpin, 7035.

H. rhodesiana, S. Moore.

Journ. Bot. 1900, 201 ; Fl. Trop. Afr. v. 509.

Salisbury, July, Rand, 519; Gwibi Flats, Sep. Eyles 413.

7929-Mellera lobulata, S. Moore.

Fl. Trop. Afr. v. 50.

Chirinda, 3700-4000 ft. June, Swyn. 1141.

7932-Phaylopsis (Phaulopsis) longfolia, T. Thoms.

Fl. Trop. Afr. v. 84 ; Fl. Cap. V. i. 21.

Victoria Falls, Sep. Gibbs, 160 ; Melsetter, 6000 ft. June, Swyn. 1929 ; Chirinda, 3700-4000 ft. Sep. Swyn. 1930.

P. parviflora, Willd.

Fl. Trop. Afr. v. 83 ; Fl. Cap. V. i. 20 ; Nat. Pl. 255.

Victoria Falls, Engler ; Rogers, 5021; Odzani River Valley, Umtali, Teague, 147.

7934-Petalidium sp.?.

Matopos, Rogers, 5177.

7939-Dyschoriste alba, S. Moore.

Journ. Bot. 1907, 89.

Mazoe, 4300-4800 ft. Jan. Eyles, 237. 
D. depressa, Nees.

Fl. Cap. V. i. 16 ; Fl. Trop. Afr. v. 72.

Victoria Falls, Rogers, 7408*.

D. Fischeri, Lindau.

Fl. Trop. Afr. v. 77 ; Fl. Cap. V. i. 17.

Bulawayo, Dec. Jan. May, Rand, 181, 182, 383; Nov. Eyles, 1160 ; Monro, 93 ; Rogers, 5733.

D. matopensis, N. E. Br.

Kew Bull. 1906, 166.

Matopos, Cecil, 114.

D. Monroi, S. Moore.

Journ. Bot. 1910, 251.

Victoria, Monro, 950 ; Bulawayo, Gardner, 98.

D. Perrottetii, O. Kuntze.

Fl. Trop. Afr. v. 72.

Victoria Falls, Sep. Gibbs, 296; Sep. Galpin, 7036 ; Engler ;

Rogers, 7138a.

D. radicans (T. And.), O. Kuntze.

North of Hartley, Engler.

D. sp.

Matopos, March, Flanagan, 2991.

7940-Disperma densiflorum, C. B. Cl.

Fl. Trop. Afr. v. 82.

Mazoe, March, Flanagan, 3028.

D. quadrisepalum, C. B. Cl. var. grandifolium, S. Moore. Journ. Bot. 1908, 73 ; Flor. Trop. Afr. v. 80 (type).

Mazoe, 4500 ft. Jan. Eyles, 513.

D. viscidissium, S. Moore.

Journ. Linn. Soc. Bot. xxxvii. 460.

Victoria Falls, Sep. Gibbs, 123.

7941-Chætacanthus Persoonii, Nees.

Fl. Cap. V. i. 18.

Matopos, 5000 ft. Nov. Eyles, 1104 ; Chirinda, 3800 ft. April, Swyn. 377.

7945-Hemigraphis myostiflora, Stapf.

Victoria Falls, Allen, 180, 412.

H. prunelloides, S. Moore.

Journ. Linn. Soc. Bot. xxxvii. 459.

Victoria Falls, Sep. Gibbs, 161 ; Engler ; Rogers, 5279, 5292, 5224 ; July, Kolbe, 3146 ; Sep. Galpin, 7037.

7953-Strobilanthopsis hircina, S. Moore.

Fl. Trop. Afr. v. 511; Journ. Bot. 1900, 202.

Fort Gibbs, Sep. Rand, 640. 
Genus No.

7965-Ruellia ovata, Thunb.

Fl. Cap. V. i. 14.

Chirinda, 3500 ft. Nov. Swyn. 1931; Victoria, Monro, 623; Bulawayo, Rogers, 13592*.

R. patula, Jacq.

Fl. Trop. Afr. v. 45 ; Fl. Cap. V. i. 12 ; Cat. Afr. Pl. Welw. i. 808. Bulawayo, Jan. Rand, 177, 178; Monro, 21 ; Oct. Eyles, 1071 ; Jan. Gardner, 31, 41 ; Matabeleland, Elliott ; Salisbury, Rand, 1369, 1382 ; Victoria, Monro, 652, 849.

R. praetermissa, Lindau.

Fl. Trop. Afr. v. 45.

Victoria Falls, Allen, 62.

7966-Eranthemum subviscosum, C. B. Cl.

Fl. Trop. Afr. v. 173.

Chirinda Forest, 3700-4000 ft. Oct. Swyn. 130.

7971-Lepidagathis scariosa, Nees.

Fl. Trop. Afr. v. 122.

Victoria Falls, Rogers, 7217*.

7972-Crabbea nana, Nees. (C. crisioides, Nees.)

Fl. Trop. Afr. v. 118 ; Fl. Cap. V. i. 38.

Bulawayo, May, Rand, 428; Victoria Falls, April, Flanagan, 3176; Chirinda, 3800 ft. April, Swyn. 304 ; Salisbury, Rogers, 5800 ; Selukwe, Rogers, 4091.

C. velutina, S. Moore.

Fl. Trop. Afr. v. 119.

Victoria Falls, Rogers, 5399, 5763.

7973-Barleria albostellata, C. B. Cl.

Fl. Trop. Afr. v. 162.

Shashi Riv. Holub, 1397 ; Matopos, Rogers, 5637*, 7908*.

B. Boehmii, Lindau.

Fl. Trop. Afr. v. 167.

Victoria Falls, Allen, 75.

B. Eylesii, S. Moore.

Journ. Bot. 1905, 50.

Matopos, 4500 ft. Feb. Eyles, 160 ; Davy; Victoria, Monro, 805.

B. Mackenii, Hook. $f$.

Matabeleland, Elliott; Bulawayo, Dec. Rand, 117; Victoria, Monro, 955.

B. matopensis, S. Moore.

Journ. Bot. 1907, 91.

Matopos, 5000 ft. Feb. Eyles, 1165 ; Victoria Falls, Rogers, 5527, 5680 . 
B. meyeriana, Nees.

Fl. Cap. V. i. 53.

Victoria Falls, Allen, 109.

B. Randi, S. Moore.

Fl. Trop. Afr. v. 512 ; Journ. Bot. 1900, 203.

Bulawayo, Dec. Rand, 115; Nov. Eyles, 88; Rogers, 5663.

B. spinulosa, Klotzsch.

Fl. Trop. Afr. v. 152.

Bulawayo, 4500 ft. Feb. Eyles, 1199 ; Victoria, Monro, 954.

B. spp.

Victoria Falls, Allen, 398, cf. B. spinulosa, Klotzsch.

Victoria Falls, Allen, 406.

Matopos, Rogers, 5264, 5412.

7978-Sclerochiton haryeyanus, Nees.

Fl. Cap. V. i. 36.

Chirinda Forest, 3700-4000 ft. \& Mt. Pene, fl. May, Oct. Dec. Swyn. 424, 455, 6119, 6638.

7980-Blepharis Bainesii, S. Moore.

Fl. Trop. Afr. v. 98.

Bulawayo, May, Rand, 381.

B. boerhaaviæfolia, Pers.

Fl. Trop. Afr. v. 96 ; Fl. Cap. V. i. 24 ; Cat. Afr. Pl. Welw. i. 811.

Victoria Falls, April, Flanagan, 3168 ; Victoria, Monro, 938.

B. diversispina, C. B. Cl.

Fl. Trop. Afr. v. 104 ; Fl. Cap. V. i. 31 ; Cat. Afr. Pl. Welw. i. 813.

Shashi Riv. Jan. and Bulawayo, May, Rand, 116, 380 ; Victoria, Monro, 398, 977.

B. innocua, C. B. Cl.

Fl. Cap. V. i. 25.

Bulawayo, Jan. Rand, 281.

B. molluginifolia, Pers.

Fl. Cap. V. i. 24 ; Fl. Trop. Afr. v. 98.

Bulawayo, Rogers, 13634*.

B. spp.

Victoria Falls, April, Flanagan, 3285, 3245.

Victoria Falls, Rogers, 5110, cf. B. pungens, Klotzsch.

Salisbury, March, Flanagan, 3026.

Matopos, 4800 ft. Feb. Eyles, 1164 ?.

Gwelo, Sr. Phil. 46 ? sp. n. 
Genus No.

8007-Äsystasia coromandeliana, Nees.

Fl. Trop. Afr. v. 131 ; Fl. Cap. V. i. 42 ; Cat. Afr. Pl. Welw. i. 817 .

Victoria Falls, 3000 ft. May, Eyles, 141; April, Flanagan, 3235 ;

Sep. Gibbs, 119.

8026-Peristrophe usta, C. B. Cl.

Fl. Trop. Afr. v. 244.

Salisbury, Sep. Rand, 507; Mazoe, 4300 ft. Aug. Eyles, 189 ;

North of Hartley, Engler.

P. sp.

Matopos, 4600 ft. March, Eyles, 1025. cf. P. caulopsila, Presl.

8030-Macrorungia pubinervia, C. B. Cl.

Fl. Trop. Afr. v. 255.

Chirinda Forest, 3700-4000 ft. May, Oct Swyn. 77, 77a.

8031-Dicliptera cephalantha, S. Moore.

Journ. Linn. Soc. Bot. xl. 162.

Chirinda, on cultivated ground, $3800 \mathrm{ft}$. May, Swyn. 514.

D. Melleri, Rolfe. (Diapedium Melleri, S. Moore.)

Fl. Trop. Afr. v. 261 \& 515.

Matabeleland, Oates, Salisbury, Sep. Rand, 507b; Mazoe, 4300 ft. Aug. Eyles, 188; Matopos, Oct. Gibbs, 80 ; Victoria, Monro, 474.

D. Monroi, S. Moore.

Journ. Bot. 1911, 189.

Victoria, Monro, 1039.

D. nobilis, S. Moore.

Journ. Linn. Soc. Bot. xl. 164.

Chirinda, 3500 ft. June, Swyn. 1935; Melsetter, 6000 ft. Sep. Swyn. 1936.

D. Swynnertonii, S. Moore.

Journ. Linn. Soc. Bot. xl. 163.

Chirinda Forest, 3700-4000 ft. May, June, July, Swyn. 528, 528a, $528 b$.

D. tanganyikensis, C. B. Cl.

North of Hartley, Engler.

8032 - Hypoëstes aristata, $R . B r$.

Fl. Trop. Afr. v. 245 ; Fl. Cap. V.i. 86.

Umtali, Engler; Chirinda, 3800 ft. May, Swyn. 105.

H. verticillaris, $R$. $\mathrm{Br}$.

Fl. Trop. Afr. v. 250 ; Fl. Cap. V. i. 88.

Bulawayo, May, Rand, 373 ; Mazoe, 4300 ft. April, Eyles, 304 ;

Sep. Eyles, 430 ; March, Flanagan, 3013 ; Salisbury, Darling ;

Gwelo, Sr. Phil. 27 ; Upper Buzi, 3000 ft. April, Swyn. 1932. 
Genus No.

8049-Siphonoglossa tubulosa, Lindau. (Justicia prostrata, Nees.)

Fl. Cap. V. i. 74.

Victoria, Monro, 822 ; Salisbury, Engler?.

8054-Rhinacanthus communis, Nees.

Fl. Trop. Afr. v. 224 ; Fl. Cap. V. i. 78 ; Cat. Afr. Pl. Welw. i. 823 .

Matengwe Riv. Holub, 1285, 1286 ; Victoria Falls ?, Rogers, 5104.

8079-Isoglossa mossambicensis, Lindau.

Fl. Trop. Afr. v. 231.

Chirinda Forest, 3700-4000 ft. May, Swyn. 248a, 1146.

8094-Justicia Bagshawei (S. Moore), Torre \& Harms. (Adhatoda Bagshawei, S. Moore.)

Journ. Bot. 1907, 333.

Chirinda Forest, 3700-4000 ft. fl. \& fr. Oct. Swyn. 127, $127 a$.

J. Betonica, $L$.

Fl. Trop. Afr. v. 184 ; Fl. Cap. V. i. 57.

Bulawayo, May, Rand, 375; Chirinda, Chimanimani Mts. $7000 \mathrm{ft.}$ Mt. Pene, 7000 ft. fl. June, July, Sep. Oct. Swyn. 136, 1121, 1987, 6120 .

J. betonicoides, $C . B . C l$.

Fl. Trop. Afr. v. 184 ; Fl. Cap. V. i. 58.

Mazoe, $5000 \mathrm{ft}$. March, Eyles, 254 ; Plumtree, April, Flanagan, 3178 ; Gwelo, Sr. Phil. 25.

J. debillis, Vahl. (Monechma bracteatum, Hochst.)

Fl. Trop. Afr. v. 214 ; Fl. Cap. V. i. 68.

South Rhodesia, Rand, 374; Redbank, April, Flanagan, 3189 ; Gwai, April, Flanagan, 3296; Gwelo, Sr. Phil. 9; Bulawayo, Rogers, 5751; Victoria Falls, Rogers, 7024*, 7199*.

J. elegantula, S. Moore.

Fl. Trop. Afr. v. 513 : Journ. Bot. 1900, 204 ; includes also vars. elatior \& repens, S. Moore, see Journ. Linn. Soc. Bot. xxxvii. 461, 462.

Salisbury, Sep. Rand, 508, 509, 642 ; Rogers, 4023 ; Bulawayo, Dec. Rand, 179 ; Matopos, 4800 ft. Nov. Eyles, 1101 ; Sep. Oct. Gibbs, 32 ; Somabula, May, Flanagan, 3266; Victoria, Monro, 399, 430, 959.

J. exigua, S. Moore.

Fl. Trop. Afr. v. 514 ; Fl. Cap. V. i. 66 ; Journ. Bot. 1900, 204. Bulawayo, May, Rand, 389.

J. Eylesii (S. Moore), Torre \& Harms. (Adhatoda Eylesii, S. Moore.) Journ. Bot. 1910, 253.

Mazoe, 4500-4800 ft. Jan. Eyles, 560. 
Genus No

\section{J. filifolia, Lindau.}

Fl. Trop. Afr. v. 198.

Victoria, Monro, 896.

J. flava, Vahl.

Fl. Trop. Afr. v. 190; Fl. Cap. V. i. 58; Cat. Afr. Pl. Welw. i. 820 .

Bulawayo, Dec. Rand, 101 ; Victoria Falls, April, Flanagan, 3241, forma.

J. glabra, Roxb.

Fl. Trop. Afr. v. 208.

South Rhodesia, Victoria Falls, Rogers, 5066.

J. matammensis, Oliv.

Fl. Trop. Afr. v. 209 ; Fl. Cap. V. i. 66.

Matabeleland, Elliott; Bulawayo, Jan. Gardner, 15 ; Matopos, March, Flanagan, 2954 ; Victoria Falls, Rogers, 5374 ; Odzani River Valley, Umtali, Teague, 145.

J. Melampyrum, S. Moore.

Fl. Trop. Afr. v. 199.

Chirinda, 3700-4000 ft. May, Swyn. 505, 506.

J. pulegioides, E. Mey. (J. protracta, T. And.)

Fl. Cap. V. i. 62 ; Nat. Pl. 216 ; Journ. Bot. 1911, 243.

Salisbury, Rand, 1380.

J. rhodesiana, S. Moore.

Journ. Bot. 1913, 188.

Bulawayo, Rogers, 5740.

J. uncinulata, Oliv.

Fl. Trop. Afr. v. 210.

Victoria, Monro, 853.

J. spp.

Salisbury, Darling, in Herb. Bolus, 11195, 11196.

Bulawayo, Feb. Eyles, 1197.

\section{Series RUBIALES.}

FAmily CCLXX.-RUBIACEAE, B. Juss.

8136-Oldenlandia angolensis, K. Schum.

Cat. Afr. Pl. Welw. i. 449.

South Rhodesia, Rand, 119. 
Fl. Trop. Afr. iii. 53 ; Cat. Afr. Pl. Welw. i. 440.

Matopos, Galpin, 6953; March, Flanagan, 2980 ; Salisbury, Rand, 474; Victoria, Monro, 845; Gwelo, Sr. Phil. 37 ; Chirinda, fl. \& fr. May, Swyn. 500 ; South Rhodesia, Rand, 118; Odzani River Valley, Umtali, Teague, 197.

0. caffra, $E . \& Z$.

Fl. Trop. Afr. iii. 58; Fl. Cap. iii. 10 ; Cat. Afr. Pl. Welw. i. 444 .

Bulawayo, 4500 ft. Nov. Eyles, 1220 ; Salisbury, May, Flanagan, 3255 ; Victoria, Monro, 851; Victoria Falls, Rogers, 5531.

0. capensis, Linn. $f$. (Hedyotis capensis, Lam.)

Fl. Trop. Afr. iii. 62 ; Fl. Cap. iii. 9; Cat. Afr. Pl. Welw. i. 446. South Rhodesia, Rand, 336.

0. cuspidata, K. Schum.

Cat. Afr. Pl. Welw. i. 443.

Gwai Forest, $3200 \mathrm{ft}$. Allen, 241.

0. cynanchica, K. Schum.

Melsetter, $6000 \mathrm{ft}$. Oct. Swyn. 6107.

0. decumbens, Hiern. (Hedyotis decumbens, Hochst.)

Fl. Trop. Afr. iii. 54; Fl. Cap. iii. 11; Cat. Afr. Pl. Welw. i. 442 .

Victoria Falls, April, Flanagan, 3166.

0. Heynei, Oliv. (Hedyotis Heynei, R. Br.)

Fl. Trop. Afr. iii. 59 ; Fl. Cap. iii. 10.

Mazoe, 4600 ft. March, Eyles, 264 ; Bulawayo, Jan. Gardner, 73 ; Matopos, March, Flanagan, 2969 ; Victoria Falls, Rogers, 6026a; South Rhodesia, Rand, 335; Odzani River Valley, Umtali, Teague, 271.

0. hirtula, O. Kuntze. (Hedyotis hirtula, Harv.)

Fl. Cap. iii. 12.

Mazoe, 5100 ft. April, Eyles, 346; Chimanimani Mts. 7000 ft. Sep. Swyn. 2152.

0. lasiocarpa, Hiern.

Fl. Trop. Afr. iii. 55.

Victoria Falls, April, Flanagan, 3243; Gwai, April, Flanagan, 3288.

0. natalensis, O. Kuntze. (Hedyotis natalensis, Hochst.)

Fl. Cap. iii. 12.

Chirinda, 3800 ft. fl. \& fr. July, Swyn. 303.

0. obtusiloba, Hiern.

Fl. Trop. Afr. iii. 56.'

Mazoe, 4400 ft. April, Eyles, 315. 
Genus No.

0. papillosa, K. Schum.

Cat. Afr. Pl. Welw. i. 443.

Salisbury, Rand, 120.

0. rhodesiana, S. Moore.

Salisbury, Rand, 122.

0. thymifolia, Prantl. (Hedyotis thymifolia, Presl.)

Fl. Cap. iii. 11.

Bulawayo, Monro, 56 ; Rand, 121, 369; Odzani River Valley, Umtali, Teague, 260.

0. trinervia, Retz.

Fl. Trop. Afr. iii. 63 ; Cat, Afr. Pl. Welw. i. 449.

Chimanimani Mts. 7000 ft. Sep. Swyn. 1534.

0. Welwitschii, Hiern.

Cat. Afr. Pl. Welw. i. 442.

Gwelo, Jan. Gardner, 14.

0. sp.

Matopos, March, Flanagan, 2981.

8154-Pentas carnea, Benth. (Neurocarpaa lanceolata, R. Br.)

Fl. Trop. Afr. iii. 46 ; Cat. Afr. Pl. Welw. i. 438.

Salisbury, March, Flanagan, 3018 ; Rogers, 4072 ; Odzani River Valley, Umtali, Teague, 174.

P. nobilis, S. Moore.

Journ. Bot. 1908, 37.

Mazoe, 5000 ft. March, Eyles, 248 ; Dec. Eyles, 496.

P. purpurea, Oliv.

Fl. Trop. Afr. iii. 46.

Chirinda, 3800 ft. fl. Feb., April, June, Swyn. 274, 1152, 2143.

P. Woodii, Scott-Elliott.

Mazoe, 4500 ft. March, Eyles, 275 ; Salisbury, Rand, 1394;

Victoria, Monro, 816, 1040 ; Gwelo, Sr. Phil. 30.

8156-Otomeria dilatata, Hiern.

Fl. Trop. Afṛ. iii. 50 ; Cat. Afr. Pl. Welw. i. 440.

Upper Buzi, 3000 ft. fl. \& fr. Nov. Dec. Swyn. 355, 2144 ; also Umswirizwi Riv., Chipinga, etc. Swyn.

8160-Dirichletia pubescens, Klotzsch.

Fl. Trop. Afr. iii. 51.

Victoria Falls, Allen, 106; Matopos, Rogers, 5414, 5533.

8212-Crossopteryx kotschyana, Fenzl.

Fl. Trop. Afr. iii. 44 ; Cat. Afr. Pl. Welw. i. 437.

Bulawayo, Rand, 415; Govt. Herb. 1076; Victoria Falls, Rogers, 5529. 
Fl. Trop. Afr. iii. 40: Cat. Afr. Pl. Welw. i. 434; For. Fl. Port. E. Afr. 70.

Upper Inyamadzi, 3300 ft. Dec. Swyn. 26; also Nyahodi, Lusitu, Upper Umswirizwi and between Umtali and Melsetter, Swyn.

8230-Cephalanthus natalensis, Oliv.

Lusitu Riv. 4000 ft. Sep. Swyn. 645; also Mt. Pene and Chimanimani Mts. Swyn.

8238-Mussaenda arcuata, Poir.

Fl. Trop. Afr. iii. 68; Cat. Afr. Pl. Welw. i. 453.

Chirinda Forest, 3800 ft. fl. \& young fr. March, Swyn. 6636.

8255-Urophyllum symplocoides, S. Moore.

Journ. Linn. Soc. Bot. xl. 79.

Mt. Pene Forest, 6500 ft. Sep. Swyn. 1278.

8274-Leptactinia lanceolata, $K$. Schum.

Mazoe, 5200 ft. Jan. Eyles, 519; Salisbury, Rand; Victoria, Monro, 421.

8283-Randia yestita, S. Moore.

Journ. Bot. 1911, 150.

Salisbury, Rand, 1395 ; Victoria, Monro.

8285-Gardenia asperula, Stapf \& Hutch.

Melsetter, 3500 ft. Oct. Swyn. 56 ; Chikore Hills, Nov. Swyn. $56 a$; Victoria, Monro, 384.

G. Neuberia, $E . \& Z$.

Fl. Cap. III. 6 ; For. Fl. Cape, 234.

Umvumvumvu Riv. 4000 ft. April, Swyn. 1401.

G. Norae Swynnerton.

Journ. Linn. Soc. Bot. xl. 80.

Chirinda Forest, 3700-4000 ft. Jan. Swyn. 11.

G. posoquerioides, S. Moore.

Journ. Linn. Soc. Bot. xl. 81.

Chirinda Forest, 3700-4000 ft. Nov. Jan. Swyn. 71, 6504.

G. resiniflua, Hiern.

Fl. Trop. Afr. iii. 102 ; For. Fl. Port. E. Afr. 73.

Victoria Falls, Allen, 59.

G. rothmannia, Linn. $f$.

Victoria, Monro, 733, 1051.

G. spatulifolia, Stapf \& Hatch.

Sabi Riv. 1000 ft. Nov. Swyn. 715. 
Genus No.

G. thunbergia, Linn. $f$.

Fl. Trop. Afr. iii. 100 ; Fl. Cap. iii. 6; For. Fl. Port. E. Afr.

72 ; For. Fl. Cape, 234 ; Nat. Pl. 40.

Sebakwe, 4000 ft. Sep. Eyles, 175 ; Umtali, Engler.

G. tigrina, Welw.

Cat. Afr. Pl. Welw. i. 462.

Chirinda Forest, 3700-4000 ft. Oct. Swyn. 10.

G. spp.

Salisbury, Rand, 570.

Shangani, Govt. Herb. 958.

Lomagundi, Govt. Herb. 986.

8293-0xyanthus Gerrardi, Sond. ?.

Fl. Cap. iii. 3 ; For. Fl. Cape, 235.

Chirinda Forest, 3700-4000 ft. fl. \& fr. Jan. Feb. Swyn. 116.

0. Swynnertonii, S. Moore.

Journ. Linn. Soc. Bot. xl. 82.

Chirinda Forest, 3700-4000 ft. fl. Oct.-Dec. fr. Dec. Swyn. 76.

var. breviflorus, S. Moore.

Journ. Linn. Soc. Bot. xl. 83.

Chirinda Forest, Nov. Swyn. 76a.

8299-Feretia æruginescens, Stapf.

Kew Bull. 1906, 79.

Victoria Falls, Allen, 57.

8303-Empogona Allenii, Stapf.

Kew Bull. 1906, 79.

Victoria Falls, Oct. Nov. Allen, 55.

8308-Tricalysia ligustrina, S. Moore.

Journ. Linn. Soc. Bot. xl. 84.

Upper Buzi, 3000 ft. Sep. Oct. Swyn. 685, 1284.

T. myrtifolia, S. Moore.

Journ. Linn. Soc. Bot. xl. 83.

Upper Inyamadzi Valley, 3000 ft. Sep. Swyn. 1133; Upper Buzi Riv. 3500 ft. Oct. Swyn. 1283.

T. pachystigma, K. Schum. (T. jasminiflora, Hook.; Coffea Engleri, K. Krause.)

Fl. Trop. Afr. iii. 124 ; Journ. Bot. 1907, 199.

Bulawayo, Rand, 637 ; Matopos, Sep. Oct. Gibbs, 67 ; Galpin, 7085 ; Engler; Chimanimani Mts. 6500 ft. Sep. Swyn. 641, 1134 ; Mt. Pene, 6500-7000 ft. Oct. Swyn. 6068.

8326-Heinsia jasminiflora, $D C$.

Fl. Trop. Afr. iii. 81 ; For. Fl. Port. E. Afr. 71.

Chirinda Forest, 3700 ft. Nov. Dec. Swyn. 1258. 
Genus No.

8337-Heinsenia sylvestris, S. Moore.

Journ. Linn. Soc. Bot. xl. 85.

Chirinda Forest, 3700-4000 ft. Oct. Swyn. 576, 689.

8338-Cremaspora africana, Benth.

Fl. Trop. Afr. iii. 126 ; Cat. Afr. Pl. Welw. i. 471.

Chirinda Forest, 3700-4000 ft. Dec. Swyn. 1285; also Chipete Forest, Swyn.

8348-Pentanisia rhodesiana, S. Moore.

Salisbury, Rand, 575, 1396.

P. Schweinfurthii, Hiern.

Fl. Trop. Afr. iii. 131.

Melsetter, $6000 \mathrm{ft.}$. Mt. Pene, $7000 \mathrm{ft.}$., North Melsetter, 5000 6000 ft. in. fl. April, Oct. Swyn. 2146, 6103, 6104, 6213.

P. sericocarpa, S. Moore. (P. crassifolia, K. Krause.)

See Journ. Bot. 1907, 198.

South Rhodesia, Rand, 619; North of Hartley, Engler; Salisbury, Sep. Engler, 3022.

P. variabilis, Harv.

Fl. Cap. iii. 24 ; Harv. Gen. S.A. Pl. 154 ; Nat. Pl. 251 (var.).

Salisbury, Darling; Odzani River Valley, Umtali, Teague, 83.

var. intermedia, Sond.

Fl. Cap. iii. 24.

Chirinda, $3800 \mathrm{ft.} \mathrm{Swyn.} 425$.

8351-Vangueria apiculata, K. Schum.

Chirinda Forest, Dec. Swyn. 64; also along eastern border of South Melsetter, Swyn.

V. esculenta, S. Moore.

Journ. Linn. Soc. Bot. xl. 91.

Chirinda Forest, 3700-4000 ft. Sep. Oct. Swyn. 65.

var. glabra, S. Moore.

Journ. Linn. Soc. Bot. xl. 91.

Chirinda Forest, Dec. Swyn. 1307.

Y. infausta, Burch.

Fl. Trop. Afr. iii. 147 ; Fl. Cap. iii. 13; Cat. Afr. Pl. Welw. i. 480 ; Nat. Pl. 369 ; For. Fl. Port. E. Afr. 75 ; For. Fl. Cape, 243.

Victoria Falls, Rogers, 5615, 5471; Chirinda, Swyn. 63 ; Chikore Hills, Swyn. 1290 ; fl. Oct. Dec. Swyn.

var. virescens, Sond. (V. edulis, Vahl, var. Bainesii, Hiern.)

Fl. Cap. iii. 14 ; see note Fl. Trop. Afr. iii. 148.

Mangwe Riv. Baines; Victoria Falls, Rogers, 5388 ; Bulawayo, Chubb $3 a$. 
Genus No.

У. munjiro, S. Moore.

Journ. Linn. Soc. Bot. xl. 92.

Victoria, Monro, $605 a$.

Y. Randii, S. Moore.

Journ. Bot. 1902, 252.

Bulawayo, Rand, 123; Monro, 97; Victoria, Monro, 692; Matabeleland, Eyles, 1191.

Y. rhodesiana, S. Moore.

Journ. Bot. 1909, 130.

Salisbury, Rand, 1349.

Y. velutina Hiern.

Fl. Trop. Afr. iii. 151 ; For. Fl. Port. E. Afr. 75.

Mazoe, 5300 ft. Dec. Eyles, 201; Victoria Falls, Allen, 415; Victoria, Monro, 639.

\section{Y. spp.}

Victoria Falls, Allen, 184, cf. V. parvifolia, Sond.

Victoria, Monro, 548, 753a, 768.

8352-Plectronia abbreviata, K. Schum. (Canthium abbreviatum, S. Moore.)

Matopos, Oct. Gibbs, 278.

P. ciliata, Sond.

Fl, Cap. iii. 18; For. Fl. Cape, 241.

Victoria Falls, Rogers, 5587 (var.).

P. Gueinzii, Sim. (Canthium Gueinzii, Sond.)

Fl. Cap. ii. 16 ; For. Fl. Cape, 241.

Chipete Forest, April, Swyn. 170; Mt. Pene, 7000 ft. fr. Oct. Swyn. 6101 ; also at Chikore and near Chirinda, Swyn.

P. lanciflora (Hiern), Torre \& Harms. (Canthium lanciflorum; Hiern.)

Fl. Trop. Afr. iii. 146.

Victoria Falls, Kirk; Salisbury, Rand, 552.

P. livida (Hiern), Torre \& Harms. (Canthium lividum, Hiern.)

Fl. Trop. Afr. iii. 144.

Victoria, Monro, 796.

P. Oatesii (Rolfe), Torre \& Harms. (Canthium Oatesii, Rolfe.) Matabeleland, Oates.

P. Randii (S. Moore), Torre \& Harms. (Canthium Randii, S. Moore.) Journ. Bot. 1911, 152.

Salisbury, Rand, 1393; Victoria, Monro, 691.

P. Swynnertonii (S. Moore), Torre \& Harms. (Canthium Swynnertonii, S. Moore.)

Journ. Linn. Soc. Bot. xl. 88.

Chirinda Forest, 3700-4000 ft. Nov. Swyn. 546. 
Genus No.

P. ventosa, $L$. (Canthium ventosum, S. Moore.)

Fl. Cap. iii. 17 ; Cat. Afr. Pl. Welw. i. 369 ; For. Fl. Cape, 240. Mt. Pene, 6500 ft. Sep. Swyn. 547.

P. sp.

Gwai Forest, 3000 ft. Allen, 250.

8354-Craterispermum laurinum, Benth.

Fl. Trop. Afr. iii. 160 ; Cat. Afr. Pl. Welw. i. 485.

Chirinda, Oct. Swyn. 183.

8359-Pachystigma Cienkowskii (Schweinf.), Torre \& Harms. (Fadogia Cienkowskii, Schweinf.)

Fl. Trop. Afr. iii. 154; Cat. Afr. Pl. Welw. i. 481.

Chirinda Forest, Swyn. 241; Mt. Pene, Swyn. 6122, 6502; in fl. Sep.-Dec. Swyn.

P. lateritica (K. Kranse), Torre \& Harms. (Fadogia lateritica, K. Krause.)

North of Hartley, Engler.

P. obovata (N. E. Br.), Torre \& Harms. (Fodogia obovata, N. E. Br.) Kew Bull. 1906, 105.

Salisbury, Cecil, 141; Rand, 1348; Mazoe, 4300 ft. Dec. Eyles, 209.

P.stenophylla (Welw.), Torre\& Harms. (Fadogia stenophylla, Welw.) Fl. Trop. Afr. iii. 155; Cat. Afr. Pl. Welw. i. 483.

Salisbury, Rand, 629, 1370.

P. tetraquetra (K. Krause), Torre \& Harms. (Fadogia tetraquetra, K. Krause.)

Umtali, Sep. Engler, 3139.

P. Zeyheri, Sond. (Fadogia Zeyheri, Sond.; Vangueria Zeyheri, Sond.)

Fl. Trop. Afr. iii. 153 ; Fl. Cap. iii. 15.

Between Bulawayo and Victoria Falls, Davy; Lomagundi, Govt. Herb. 990.

8360-Ancylanthus Bainesii, Hiern.

Fl. Trop. Afr. iii. 160.

Victoria Falls, Allen, 97.

8381-Coffea ligustroides, S. Moore.

Journ. Linn. Soc. Bot. xl. 94.

Chipete Forest, 3800 ft. fl. Dec. ripe fr. July, Swyn. 67.

C. Swynnertonii, S. Moore.

Journ. Linn. Soc. Bot. xl. 95.

Chirinda, $3000 \mathrm{ft}$. in cultivation, fl. Oct. fr. Dec, Swyn. 578.

8383-Pavetta angolensis, Hiern.

Cat. Afr. Pl. Welw. i. 485.

Victoria, Monro, 666 ; Matopos, Rogers, 5260. 


\section{P. assimilis, Sond.}

Fl. Cap. iii. 20.

Sebakwe, 4000 ft. Dec. Eyles, 168; Bulawayo, Monro, 49;

Victoria, Monro, 806.

P. Cecilae, N. E. Br.

Kew Bull. 1906, 106.

Selukwe, Cecil, 124 ; Mazoe, 5300 ft. Dec. Eyles, 203.

P. comostyla, S. Moore.

Journ. Linn. Soc. Bot. xl. 98.

Chiranda Forest, 3700-4000 ft. Jan. Swyn. 75.

P. Eylesii, S. Moore.

Journ. Bot. 1905, 47.

Matopos, 4500-5000 f. Nov. Eyles, 1159.

P. gardeniæfolia, Hochst.

Fl. Trop. Afr. iii. 177.

South Rhodesia, Rand, 421.

P. luteola, Stapf.

Kew Bull. 1906, 80.

Victoria Falls, Oct. Allen, 54.

P. neurophylla, S. Moore.

Journ. Bot. 1905, 47.

Bulawayo, 4500 ft. Dec. Eyles, 1140 ; Monro, 52.

P. obovata, E. Mey.

Fl. Cap. iii. 20 ; Nat. Pl. 313 ; For. Fl. Cape, 237.

Bulawayo, Monro, 54.

P. schumanniana, $K$. Hoff $m$.

Cat. Afr. Pl. Welw. i. 488.

Bulawayo, Monro, 104; Victoria Falls, Rogers, 7244 ; Chikore Hills, 3500 ft. Nov. Swyn. 1212.

P. stipulopallium, $K$. Schum.

Salisbury, Rand, 1350 ; Victoria, Monro, 666, 1056a; Nyahodi Riv. 5000 ft. April, Swyn. 2147.

P. Swynnertonii, S. Moore. Journ. Linn. Soc. Bot. xl. 99.

Chirinda Forest, 3700-400J ft. Jan. Swyn. 117.

\section{P. spp.}

Victoria Falls, Allen, 94, ? n. sp. ; Rogers, 5049.

Victoria Falls, Rogers, 5553, cf. P. gracilis, Klotzsch.

Bulawayo, Monro, 53.

8399-Psychotria hirtella, Oliv.

Victoria Falls, Rogers, 5382.

P. sp.

Victoria, Monro, 802. 
Genus No.

8402-Grumilea punicea, S. Moore.

Journ. Linn. Soc. Bot. xl. 101.

Chimanimani Mts. 7000 ft. fl. \& unripe fr. Sep. Swyn. 563.

8410-Geophila uniflora, Hiern.

Fl. Trop. Afr. iii. 221 ; Cat. Afr. Pl. Welw. i. 498.

Chirinda Forest, 3700-4000 ft. March, Swyn. 350.

8435-Galopina circæoides, Thunb.

Fl. Cap. iii. 26.

Chirinda, fl. \& fr. April, Swyn. 317.

8438-Anthospermum ammanioides, S. Moore.

Journ. Linn. Soc. Bot. xl. 102.

Melsetter, $6000 \mathrm{ft}$. April, Swyn. 2156.

A. ciliare, $L$.

Fl. Cap. iii. 28.

Matopos, Oct. Gibbs, 186.

A. hispidulum, E. Mey.

Fl. Cap. iii. 29.

Mazoe, $5100 \mathrm{ft}$. April, Eyles, 343.

A. lanceolatum, Thunb.

Fl. Cap. iii. 30.

Matopos, Sep. Gibbs, 93 ; Odzani River Valley, Umtali, Teague, 181.

A. Randii, S. Moore.

Salisbury, Rand, 475 ; Victoria, Monro, 326.

A. rigidum, $E . \& Z$.

Fl. Cap. iii. 31.

Victoria, Monro, 884 ; South Rhodesia, Rand, 338; Bulawayo, Rogers, 13537*.

A. vallicolum, S. Moore.

Journ. Linn. Soc. Bot. xl. 103.

Chimanimani Mts. 7000 ft. Sep. Swyn. 2155.

A. sp.

Salisbury, May, Flanagan, 3196.

8450-0tiophora inyangana, N. E. Br.

Kew Bull. 1906, 107.

Inyanga, Cecil, 203; Mt. Pene, 7000 ft. Sep. Oct. Swyn. 2007, 6146.

0. scabra, Zucc.

Cat. Afr. Pl. Welw i. 499 ; Journ. Bot. 1908, 76.

Mazoe, 5200 ft. Jan. Eyles, 522. 
Genus No

8464-Richardsonia brasiliensis, Hayne.

Chirinda, 3700 ft. fl. \& fr. Nov. Swyn. 2134.

R. scabra, St. Hil. (Richardia scabra, L.)

Fl. Trop. Afr. iii. 242.

Salisbury, Rand, 1397.

8473-Borreria aryensis, K. Schum. (Tardavel arvensis, Hiern.)

Cat. Afr. Pl. Welw. i. 504.

Victoria, Monro, 981, 1020.

D. dibrachiata, K. Schum. (Spermacoce dibrachiata, Oliv.; Tardavel dibrachiata, Hiern.)

Fl. Trop. Afr. iii. 239 ; Cat. Afr. Pl. Welw. i. 507.

Umtali, Engler, Odzani River Valley, Teague, 12, 119 ; Matopos, March, Flanagan, 2946 ; Mazoe, 4300-4800 ft. Jan. Eyles, 243 ; Salisbury, Rogers, 5797, Kolbe.

B. natalensis, K. Schum. (Spermacoce natalensis, Hochst.)

Fl. Cap. iii. 24.

Chirinda, May, Swyn. 376, 518.

B. Ruelliae, K. Schum. (Spermacoce Ruelliae, DC.; Tardavel scabra, Hiern.)

Fl. Trop. Afr. iii. 238; Cat. Afr. Pl. Welw. i. 504.

Mazoe, 4400-4600 ft. March, Eyles, 271; Bulawayo, Monro, 941 ; Victoria Falls, Rogers, 5081a*.

B. stricta, K. Schum. (Spermacoce stricta, Linn. f.; Tardavel stricta, Hiern.)

Fl. Trop. Afr. iii. 236 ; Cat. Afr. Pl. Welw. i. 503.

Mazoe, $4600 \mathrm{ft}$. March, Eyles, 260.

B. subvulgata, $K$. Schum.

Odzani River Valley, Umtali, Teague, 225; Victoria Falls, Rogers, 7032\%.

\section{B. spp.}

Gwai, April, Flanagan, 3193.

Salisbury, May, Flanagan, 3198.

8486-Galium stenophyllum, Baker.

Chipete, 3800 ft. fl. \& fr. July, Swyn. 2141.

8489-Rubia cordifolia, $L$.

Fl. Trop. Afr. iii. 244 ; Fl. Cap. iii. 35.

Chirinda, 3800 ft. April, Swyn. 216; Odzani River Valley, Umtali, Teague, 215.

R. petiolaris, $D C$.

Fl. Cap. iii. 35.

Bulawayo, Jan. Gardner, 8 ; Rogers, 5684. 
FAMILY CCLXXIV.-DIPSACACEAE, Lindl.

Genus No.

8546-Ścabiosa columbaria, $L$.

Fl. Trop. Afr. iii. 252 ; Fl. Cap. iii. 43 ; Cat. Afr. Pl. Welw. i. 512.

Bulawayo, 4500 ft. Jan. Eyles, 61 (var.); Salisbury, Engler; Darling; Mazoe, 4300-4800 ft. Nov. Eyles, 452 ; Victoria, Monro, 949; Chirinda, April, Swyn. 278; Mt. Pene, Sep. Swyn. 1143; South Rhodesia, Rand, 98; Odzani River Valley, Umtali, Teague, 75.

Series CAMPANULATAE.

Family CCLXXV.CUCURBITACEAE, Hall.

8562-Melothria longepedunculata, Cogn.

Chirinda Forest, 3700-4000 ft. Jan. Swyn. 232 (forma).

8591-Momordica Balsamina, $L$.

Fl. Trop. Afr. ii. 537 ; Fl. Cap ii. 491 ; Cat. Afr. Pl. Welw. i. 394 .

Victoria Falls, fl. \& fr. Sep. Gibbs, 299; Allen, 179 ; Engler ; July, Kolbe, 3135 ; Rogers, 5604, 5111.

M. clematidea, Sond.

Fl. Cap. ii. 491.

Wankie, Rogers, 13244*; Victoria Falls, Rogers, 13164*.

M. fœtida, Schum. \& Thonn.

Chirinda Forest, 3700-4000 ft. Dec. Swyn. 94.

M. involucrata, E. Mey.

Fl. Cap. ii. 491 ; Nat. Pl. 516.

Bulawayo, May, Rand, 327.

M. Morkorra, A. Rich. (M. cordifolia, Sond.)

Fl. Trop. Afr. ii. 538 ; Fl. Cap. ii. 492.

Mazoe, $4500 \mathrm{ft}$. Nov. Eyles, 473 ; Odzani River Valley, Umtali, Teague, 91.

\section{M. spp.}

Victoria Falls, Dec. Allen, 224.

South Rhodesia, Rand, 93.

8598-Citrullus vulgaris, Schrad. (Colocynthis amarissima, Schrad.)

Fl. Trop. Afr. ii. 549 ; Fl. Cap. ii. 494 ; Cat. Afr. Pl. Welw. i. 397.

Matopos, Rogers, 5659. 
A Record of Plants Collected in Southern Rhodesia.

Genus No.

8599-Cucumis Cecili, N. E. Br.

Kew Bull. 1906, 104.

Inyanga, Cecil, 225.

C. Figarei, Delile. (C. chrysocomus, Schum. \& Thonn.)

Fl. Trop. Afr. ii. 543 ; Cat. Afr. Pl. Welw. i. 396.

Victoria Falls, April, Flanagan, 3306 ; Matopos, Rogers, 5659a?.

C. hirsutus, Sond.

Fl. Trop. Afr. ii. 546; Fl. Cap. ii. 497.

Bulawayo, Jan. Rand, $90 \& 91$.

C. naudinianus, Sond.

Fl. Cap. ii. 496 in part; Fl. Trop. Afr. ii. 549.

Bulawayo, Rogers, 5739.

C. Zeyheri, Sond.

Fl. Cap. ii. 496.

Chirinda, 3800 ft. May, Swyn. 496.

C. spp.

Bulawayo, Dec. Eyles, 50 ; Chubb, 322, 328.

8608 -Trochomeria macrocarpa, Hook.f. (Zehneria macrocarpa, Sond.) Fl. Trop. Afr. ii. 524 ; Fl. Cap. ii. 488 ; Harv. Gen. S.A. Pl. 125.

Mazoe, 4600 ft. Sep. Eyles, 424; Bulawayo, Monro, 70.

8612 -Peponium (Peponia) chirindensis, Baker $f$.

Journ. Linn. Soc. Bot. xl. 74.

Chirinda, 3700-4000 ft. Nov. Swyn. 2102.

8628 -Coccinia palmata, Cogn. (Cephalandra palmata, Sond.)

Fl. Cap. ii. 493 ; Nat Pl. 283.

Bulawayo, Jan. Rand, 87 \& 89.

C. pubescens, Sond. (Cephalandra pubescens, Sond.)

Fl. Trop. Afr. ii. 551 ; Fl. Cap. ii. 493.

Bulawayo, Rogers, 5660 .

C. sp.

Bulawayo, Rogers, 5708.

FAmily CCLXXVI.-CAMPANULACEAE, Juss.

8663-Prismatocarpus spp.

Victoria Falls, Allen, 118 ; Rogers, 5617 ; April, Flanagan, 3162

8668-Wahlenbergia arenaria, $A$. $D C$.

Fl. Cap. iii. 581.

Between Salisbury and Macheke, Rogers, 4025.

W. banksiana, $A . D C$.

Fl. Cap. iii. 574.

Matabeleland, Oates. 
W. Caledonica, Sond.

Fl. Cap. iii. 579.

Matopos, 5000 ft. March, Eyles, 1065 ; March, Flanagan, 2964 ; Oct. Gibbs, 220 ; Mazoe, 4400 ft. April, Eyles, 306 ; Bulawayo, Monro, 12; Rand, 476; Victoria, Monro, 876; Inyanga, Cecil ; South Rhodesia, Rand, 358, 616.

W. mashonica, N. E. Br.

Kew Bull. 1906, 165.

Between Salisbury and Headlands, Cecil, 157.

W. Oatesii, Rolfe.

Matabeleland, Oates.

W. rhodesiana, S. Moore.

Journ. Linn. Soc. Bot. xl. 125.

Melsetter, 5000-6000 ft. fl. \& fr. Oct. Swyn. 6225.

W. rivularis, Diels?.

Victoria, Monro, 928.

W. saginoides, S. Moore.

Journ. Bot. 1911, 153.

Victoria, Monro, 649.

W. undulata, $A$. $D C$.

Fl. Cap. iii. 579 ; Nat. Pl. 37.

Gwelo, Jan. Gardner, 18 ; Bulawayo, Jan. Gardner, 71.

W. virgata, Engl.

Melsetter, April, Swyn. 2020; Mt. Pene, 6500-7000 ft. Sep. Swyn. 2021.

W. Zeyheri, E. \& $Z$.

Fl. Cap. iii. 580.

Matabeleland, Jan. Eyles, 1056; North of Hartley. Engler; Marandellas, Engler; Umtali, Engler; Victoria, Monro, 753, 909.

\section{W. spp.}

Salisbury, May, Flanagan, 3268, cf. W. paniculata, A. DC.

Salisbury, May, Flanagan, 3253.

Matopos, March, Flanagan, 2974; Eyles, 1028, 1147 ; Rogers, 5685.

Victoria Ealls, Feb. Allen, 288.

Gwai Forest, Jan. Allen, 242.

8670-Lightfootia abyssinica, Hochst.

Fl. Trop. Afr. iii. 474.

Mazoe, 4300-4800 ft. Sep. Eyles, 421 ; March, Flanagan, 3012 ; Salisbury, Rand, 478, 479; Chirinda, 3800 ft. May, Swyn. 515 ; Odzani River Valley, Umtali, Teague, 259. 
Genus No.

L. denticulata, Sond. (L. capillaris, Buek.)

Fl. Cap. iii. 559.

Matopos, 4800 ft. March, Eyles, 1038 ; March, Flanagan, 2961;

Rogers, 5685 ; Gwelo, Sr. Phil. 47.

L. glomerata, Engl.

Mazoe, 5100 ft. March, Eyles, 285; Flanagan, May, 3257.

L. juncea, Sond.

Fl. Cap. iii. 563.

Salisbury, Engler.

L. tenuifolia, $A . D C$.

Fl. Trop. Afr. iii. 475; Cat. Afr. Pl. Welw. i. 629.

Matopos, Sep. Gibbs, 35 ; Engler; Salisbury, Rand, 477 ; South Rhodesia, Rand, 359, 360.

L. sp.

Salisbury, May, Flanagan, 3229.

8681-Cyphia alba, N. E. Br.

Kew Bull. 1906, 165.

North of Umtali, Cecil, 163.

C. mazoensis, S. Moore.

Journ. Bot. 1907, 46.

Mazoe, 4700-4900 ft. Jan. Eyles, 231.

8694-Lobelia Boivini, Sond.

Fl. Cap. iii. 546.

Matopos, 5000 ft. March, Eyles, 1040.

L. cobaltica, S. Moore.

Journ. Linn. Soc. Bot. xl. 124.

Chimanimani Mts. 7000 ft. Sep. Swyn. 2036.

L. decipiens, Sond.

Fl. Cap. iii. 540.

Matabeleland, Oates; Matopos, Sep. Oct. Gibbs, 87 ; Marandellas, Engler; Rogers, 4041 ; Mt. Pene, 6000 ft. Oct. Swyn. 6081 ; Victoria, Monro, 583.

L. Erinus, $L$.

Fl. Cap. iii. 544.

Victoria Falls, Rogers, 5290.

L. fervens, Thunb.

Fl.Trop. Afr. iii. 468 ; Fl. Cap. iii. 548 ; Cat. Afr. Pl. Welw. i. 627. Chirinda, 3800 ft. May, Swyn. 513.

L. fonticola, Engl. \& Gilg.

Matopos, Sep. Gibbs, 96.

L. jugosa, S. Moore.

Journ. Linn. Soc. Bot. xl. 125.

Between Lusitu and Nyahodi Rivs. 5000 ft. Sep. Swyn. 2037 ;

Mt. Pene, Swyn. 6080 ?, 6085 ?, Victoria, Monro, 309. 
Genus No.

L. microdon, A. DC. (L. Erinus, L. var. microdon, Sond.) Fl. Cap. iii. 545 .

Matopos, Sep. Oct. Gibbs, 37.

L. minutidentata, Engl. \& Gilg. Matopos, Oct. Gibbs, 272.

L. natalensis, $A . D C$.

Fl. Trop. Afr. iii. 469 ; Fl. Cap. iii. 545.

Plumtree, April, Flanagan, 3184 ; Mazoe, 5200 ft. April, Eyles, 348; Victoria, Monro, 327 ; South Rhodesia, Rand, 172; Odzani River Valley, Umtali, Teague, 222.

L. thermalis, Thunb. (Parastranthus thermalis, Sond.)

Fl. Cap. iii. 537; Cat. Afr. Pl. Welw. i. 625.

Bulawayo, 4500 ft. Oct. Eyles, 1076 ; Monro, 71 ; Gwelo, Jan. Gardner, 10 \& 26 ; Matopos, Sep. Oct. Gibbs, 31.

L. trullifolia, Hemsl.

Fl. Trop. Afr. iii. 466.

Matopos, Oct. Gibbs, 271.

\section{L. spp.}

Salisbury, March, Flanagan, 3025.

Victoria Falls, Allen, 19, cf. L. Erimus, L. ; Rogers, 5134.

Matopos, Nov. Eyles, 1105.

\section{Family CCLXXX.-COMPOSITE, Vaill.}

8734 -Ethulia conyzoides, $L$.

Fl. Trop. Afr. iii. 262 ; Fl. Cap. iii. 47.

Victoria Falls, Allen, 83; April, Flanagan, 3035 ; Rogers, 5082. 8740-Erlangea Eylesii, S. Moore.

Journ. Bot. 1908, 38.

Mazoe, 4400 ft. April, Eyles, 309.

E. laxa, S. Moore.

Matopos, 4800 ft. April, Eyles, 43 ; Mazoe, 4800 ft. March, Eyles, 293.

E. longipes, S. Moore.

Chirinda, Swyn. 391 ; Melsetter, Swyn. 1809.

E. Schinzii, $O$. Hoffm.

Victoria Falls, Sep. Gibbs, 298.

E. spp.

Gwai, April, Flanagan, 3070.

Victoria Falls, April, Flanagan, 3063 ; Rogers, 6156.

8746-Bothriocline inyangana, $N$. E. Br.

Kew Bull. 1906, 107.

Inyanga, Cecil, $227 a$. 
Y. Bainesii, Oliv. \& Hiern.

Fl. Trop. Afr. iii. 272.

Salisbury, April, Flanagan, 3046 ; Chipetzana Riv. 4000 ft. April, Swyn. 1903; between Lusitu and Nyahodi Rivs. 5000 ft. Swyn. 1903a; Victoria, Monro, 1272; Odzani River Valley, Umtali, Teague, 176.

Y. cinerea, Less.

Fl. Trop. Afr. iii. 275 ; Cat. Afr. Pl. Welw. i. 521.

Victoria Falls, Rogers, 5042.

Y. cistifolia, O. Hoffm. var. rosea, O. Hoffm.

Chirinda, 3800 ft. May, Swyn. 496.

Y. fastigiata, Oliv. \& Hiern.

Fl. Trop. Afr. iii. 282.

Bulawayo, May, June, Rand, 277 \& 352 ; Chubb, 379 ; Gwelo, Sr. Phil. 42 ; Nyamandhlovu, April, Flanagan, 3066.

Y. gerberæformis, Oliv. \& Hiern.

Fl. Trop. Afr. iii. 285.

Salisbury, Sep. Rand, 626; Melsetter, 6000 ft. Sep. Oct. Swyn. 1900 ; Mt. Pene, 6500-7000 ft. Swyn. 6112.

Y. glabra, Vatke.

Fl. Trop. Afr. iii. 286.

Bulawayo, May, Rand, 353; Mazoe, 4300 ft. April, Eyles, 317 ; April, Flanagan, 3058 ; Victoria Falls, April, Flanagan, 3064; Chirinda, 3500 ft. April, Swyn. 1814; Chipetzana Riv. 3000 ft. Aug. Swyn. 1831 ; Salisbury, Sep. Rand, 622 (var.); Odzani River Valley, Umtali, Teague, 106.

V. gracilipes, S. Moore.

Journ. Linn. Soc. Bot. xl. 105.

Chimanimani Mts. 7000 ft. Sep. Swyn. 1830, 1830a; Mt. Pene, 6000 ft. Oct. Swyn. 6126, 6127.

var. minor, S. Moore.

Journ. Linn. Soc. Bot. xl. 106.

Chimanimani Mts. 7000 ft. Sep. Swyn. 1909.

Y. hirsuta, Sch. Bip.

Fl. Cap. iii. 51 ; Nat. Pl. 335.

North Melsetter, 5000-6000 ft. Oct. Swyn. 6116, 6138.

Y. Holstii, O. Hoffm.

Chirinda, 3700-4000 ft. June, Swyn. 523a.

Y. humilis, C. H. Wright.

Salisbury, Dec. Rand, 153 ; May, Rand, 492. 
Genus No.

8751 - $\mathbf{Y}$. integra, S. Moore.

Journ. Bot. 1908, 39.

Mazoe, 4300-4800 ft. March, Eyles, 277 ; Gwelo, Sr. Phil. 3 ; Umtali, Rogers, 4079*.

Y. Kraussii, Sch. Bip.

Fl. Trop. Afr. iii. 276 ; Fl. Cap. iii. 51 ; Nat. Pl. 334.

Bulawayo, Dec. Rand, 150 ; Salisbury, May, Rand, 624 ; Darling ; Rogers, 4080 ; Matopos, Engler ; Oct. Gibbs, 342 ; North of Hartley, Engler ; Umtali, Engler; Mt. Pene, 6000-7000 ft. Sep., Umvumvumvu Riv. 4000 ft. Oct. Swyn. 1827, 6002, 6141,6142 .

Y. lancibracteata, S. Moore.

Journ. Bot. 1908, 293.

Mazoe, 4500-5000 ft. March, Eyles, 291.

Y. livingstoniana, Hiern.

Fl. Trop. Afr. iii. 295.

Chirinda, Swyn.

Y. mashonica, N. E. Bi.

Kew Bull. 1906, 108.

Salisbury and Umtali, Cecil, 70, 229.

V. Melleri, Oliv. \& Hiern.

Fl. Trop. Afr. iii. 282.

Salisbury, Aug. Rand, 494; April, Flanagan, 3047 ; Mazoe, 4500-4800 ft. Aug. Eyles, 185; Odzani River Valley, Umtali, Teague, 97.

Y. monocephala, Harv.

Fl. Cap. iii. 53 ; Nat. Pl. 331.

Melsetter, $6000 \mathrm{ft}$. Sep. Swyn. 1829 ; Chimanimani Mts. $7000 \mathrm{ft}$. Oct. Swyn. 6144.

Y. natalensis, Sch. Bip.

Fl. Trop. Afr. iii. 277 ; Fl. Cap. iii. 51; Nat. Pl. 333 ; Cat. Afr. Pl. Welw. i. 522.

Salisbury, Engler ; Chirinda, 3800 ft. Oct. Swyn. 275 ; Mt. Pene, 7000 ft. Swyn. 6145 .

Y. pauciflora, Less.

Fl. Trop. Afr. iii. 283.

Matopos, Rogers, 5360?.

ע. Petersii, Oliv. \& Hiern.

Fl. Trop. Afr. iii. 273 ; Cat. Afr. Pl. Welw. i. 519.

Deka, 2400 ft. May, Eyles, 135. 
Fl. Trop. Afr. iii. 296 ; Cat. Afr. Pl. Welw. i. 539.

Victoria Falls, Sep. Galpin, 7029; Matopos, fl. \& fr. Sep. Gibbs, 66 ; Mazoe, 4700 ft. Aug. Eyles, 386 ; Chirinda, Sep. Swyn. 113 ; Odzani River Valley, Umtali, Teague, 258.

Y. porphyrolepis, S. Moore.

Journ. Bot. 1908, 39.

Mazoe, 4800-5000 ft. May, Eyles, 371: Odzani River Valley, Umtali, Teague, 71.

Y. Poskeana, Vatke \& Hildebr. var. chlorolepis, Steetz. (V. steetziana, Oliv. \& Hiern.)

Fl. Trop. Afr. iii. 273, 274 ; Cat. Afr. Pl. Welw. i. 520.

Bulawayo, May, Rand, 344 ; Victoria Falls, 3000 ft. May, Eyles, 137 ; Chipetzana Riv. 3000 ft. Dec. Swyn. 1828a; Mazoe, 4400 ft. April, Eyles, 311; Plumtree, April, Flanagan, 2067 ; Odzani River Valley, Umtali, Teague, 96.

Y. purpurea, Sch. Bip.

Fl. Trop. Afr. iii. 281.

Salisbury, April, Flanagan, 3042.

Y. Randii, S. Moore.

Journ. Bot. 1899, 369.

Salisbury, July, Rand, 495, 1371 ; Mazoe, 4300-4800 ft. July, Aug. Eyles, 380, 387; Victoria, Morıro, 338b, 358, 692 ; Matopos, Rogers, 5162 ; Victoria Falls, Rogers, 5295 ; Odzani River Valley, Umtali, Teague, 250.

Y. senegalensis, Less.

Fl. Trop. Afr. iii. 283 ; Fl. Cap. iii. 50 ; Cat. Afr. Pl. Welw. i. 528 ; For. Fl. Port. E. Afr. 77.

Victoria Falls, Allen, 33 ; Engler ; Rogers, 5054; Mazoe, April, Flanagan, 3052 ; Victoria, Monro, 338; Umvukwe, Govt. Herb. 1091.

Y. tenoreana, Oliv.

Fl. Trop. Afr. iii. 290.

Salisbury, July, Rand, 497.

V. Wollastonii, S. Moore.

Victoria, Monro, 1550, 1990.

Y. Woodii, O. Hoffm.

Salisbury, May, Flanagan, 3038 ; Victoria Falls, April, Flanagan, 3065 , Victoria, Monro, 346 ; Melsetter, 6000 ft. April, Swyn. 1824; Upper Buzi, 3000 ft. May, Swyn. 1824a; also Chirinda, Swyn. 
Genus No.

7851-⿱亠乂. spp.

Mazoe, April, Flanagan, 3078, ef. V. turbinata, Oliv. \& Hiern.

Victoria Falls, April, Flanagan, 3074 ; Allen, 131 ; Rogers, 5156, $5295 a, 7015$, cf. V. demulans, Vatke.

Bulawayo, Dec. Gardner, 84.

Matopos, March, Eyles, 1045; Rogers, 5171 \& 5360, ef. V. pauciflora, Less.

8775-Elephantopus scaber, $L$.

Fl. Trop. Afr. iii. 299 ; Cat. Afr. Pl. Welw. i. 540.

Mazoe, 4500-4800 ft. March, Eyles, 256.

8785-Adenostemma viscosum, Forst. (A.caffrum, DC. ; A. Dregei, DC.) Fl. Trop. Afr. iii. 259; Fl. Cap. iii. 58 ; Cat. Afr. Pl. Welw, i. 542 ; Nat. Pl. 346.

Matopos, 5000 ft. Feb. Eyles, 1168; Rogers, 5693; Mazoe, 4300 ft. Dec. Eyles, 226 ; Salisbury, May, Flanagan, 3050 ; between Salisbury and Umtali, Rogers, 4072 ; Victoria Falls, April, Flanagan, 3049.

8816-Eupatorium africanum, Oliv. \& Hiern.

Fl. Trop. Afr. iii. 301 : Cat. Afr. Pl. Welw. i. 542.

Gwelo, Jan. Gardner, 43; North of Hartley, Engler; Umtali, Engler; Mazoe, 4300-4800 ft. Sep. Eyles, 431; Salisbury, Darling in Herb. Bolus, 10779 ; Rogers, 4012 ; South Rhodesia, Rand, 153.

8818-Mikania scandens, Willd. (M. capensis, DC.; Willugbaeya scandens, O. Kuntze.)

Fl. Trop. Afr. iii. 301 ; Fl. Cap. iii. 59 ; Cat. Afr. Pl. Welw. i. 543.

Mazoe, $4400 \mathrm{ft}$. April, Eyles, 362 ; Umtali, Engler; Victoria Falls, Sep. Galpin, 6962; Rogers, 5124, 7218; Victoria, Monro, 823.

8865-Grangea maderaspatana, Poir.

Fl. Trop. Afr. iii. 304 ; Cat. Afr. Pl. Welw. i. 545.

Victoria Falls, Allen, 100.

8901-Erigeron canadense, $L$

Fl. Cap. iii. 86 ; Harv. Gen. S.A. Pl. 173.

Salisbury, Aug. Rand, 481 ; Bulawayo, 4600 ft. Aug. Eyles, 1232;

Macheke, Engler; Odzani River Valley, Umtali, Teague, 136.

8919-Felicia angustifolia, Nees.

Fl. Cap. iii. 73.

Bulawayo, Rogers, 13619*, 13668\%.

F. fascicularis, DC. (Aster muricatus, Less. var. fascicularis, E. Mey.)

Fl. Cap. iii. 72.

South Rhodesia, Rand, 347. 
A Record of Plants Collected in Southern Rhodesia.

Genas No.

F. hyssopifolia, Nees.

Bulawayo, Jan. Gardner, 79.

F. lutea, N. E. Br.

Kew Bull. 1901, 123.

Bulawayo, 4500 ft. Nov. Eyles, 1216 ; Sebakwe, 4000 ft. Dec. Eyles, 161; Victoria, Monro, 1076; Gwelo, Sr. Phil. 34; Odzani River Valley, Umtali, Teague, 118.

F. simulans? (Detris simulans?)

Salisbury, Aug. Rand. 491.

F. tenella, DC. (Aster tenellus, L.)

Fl. Cap. iii. 71.

South Rhodesia, Jan. Rand, 135.

F. sp.

Bulawayo, Chubb, 364, ef. F. rigidula, DC.

8923-Psiadia arabica, Jaub. \& Spach.

Fl. Trop. Afr. iii. 319 ; Cat. Afr. Pl, Welw. i. 553.

Bulawayo, Jan. Rand, 138.

8925-Nidorella auriculata, $D C$.

Fl. Cap. iii. 88.

Matabeleland, Oates; Matopos, Rogers, 5359; Odzani River Valley, Umtali, Teague, 4.

N. depauperata, Harv.

Fl. Cap. iii. 90.

Victoria Falls, July, Kolbe, 3144 ; Swartz.

N. hirta, $D C$.

Fl. Cap. iii. 88.

Rusapi, Engler.

N. microcephala, Steetz.

Fl. Trop. Afr. iii. 310.

Umtali, Engler; Bulawayo, Chubb, 402 ; Chirinda, 3700-4000 ft. May, Swyn. 287.

N. namaquensis?.

Matabeleland, Marloth.

N. resedifolia, $D C$.

Fl. Cap. iii. 88 ; Cat. Afr. Pl. Welw. i. 550.

Bulawayo, Dec. Rand, 137 ; Chubb, 320 p.p., 398 ; Nov. Eyles, 1110 : Dec. Gardner, 99 ; Victoria Falls, Allen, 121.

\section{N. sp.}

Matopos, Rogers, 5186.

8926-Conyza ægyptiaca, Ait. (Marsea agyptiaca, Hiern.)

Fl. Trop. Afr. iii. 314 ; Cat. Afr. Pl. Welw. i. 550.

Victoria Falls, Rogers, 5014. 
Genus No.

C. costata, Harv.

Fl. Cap. iii. 114.

Odzani River Valley, Umtali, Teague, 217.

C. paucifolia, Oliv. \& Hiern.

Victoria Falls, Rogers, 5015.

C. persicæfolia, Oliv. \& Hiern. (Marsea persicefolia, Hiern.)

Fl. Trop. Afr. iii. 312 ; Cat. Afr. Pl. Welw. i. 550.

Victoria Falls, Swartz; Chirinda, 3700-4000 ft. June, Swyn. 2066, 2065a; Odzani River Valley, Umtali, Teague, 144.

C. pinnatifida, Less.

Fl. Cap. iii. 113.

Odzani River Valley, Umtali, Teague, 249.

C. variegata, Sch. Bip.

Fl. Trop. Afr. iii. 315.

Bulawayo, May, Rand, 357 ; Melsetter, 6000 ft. Oct. Swyn. 6128.

C. sp.

Victoria Falls, Rogers, 5325. ${ }^{\mathrm{I}}$

8929-Nolletia.

Victoria Falls, Rogers, 5109, cf. N. rariflora, Steetz.

8936-Brachylæna discolor, $D C$.

Fl. Cap. iii. 117 ; Nat. Pl. 23 \& 24 ; For. Fl. Port. E. Afr. 77 ; Flor. Fl. Cape, 247.

Victoria, Monro, 573.

B. rhodesiana, S. Moore.

Journ. Linn. Soc. Bot. xxxvii. 448.

Matopos, Sep. Gibbs, 72 ; Galpin, 7069 ; Bulawayo, Chubb, 25 ;

Inyamadzi Valley, 3000 ft. Swyn. 1856; Chipetzana Riv. 3000 ft. Swyn. 1857 ; also Chikore and Lusitu, Swyn.

B. rotundata, S. Moore.

Victoria, Monro, 516.

B. spp.

Victoria, Monro, 478, 965.

8937-Tarchonanthus camphoratus, $L$.

Fl. Cap. iii. 118; For. Fl. Port. E. Afr. 77; For. Fl. Cape, 245.

South of Bulawayo, Galpin, 7008; Bulawayo, Engler; April, Eyles, 64 ; Matopos, Sep. Gibbs, 315 ; Engler; Gwelo, Sr. Phil. 52.

T. sp.

South Rhodesia, Rand, 342.

${ }^{1}$ Rogers has this named Microglossa volubilis, DC. 
Genus No.

8939-Blumea Gariepina, DC. (Placus gariepinus, O. Kuntze.)

Fl. Cap. iii. 120 ; Cat. Afr. Pl. Welw. i. 556.

Bulawayo, Dec. Rand, 278 ; Oct. Eyles, 1075 ; Chubb, 312 ; Mazoe, 4800 ft. Jan. Eyles, 235 ; Matopos, Sep. Gibbs ; Victoria Falls, Allen, 408; Galpin, 7007; Gwelo, Sr. Phil. 51 \& 55; South Rhodesia, Rand, 341.

B. lacera, DC. (Placus lacerus, O. Kuntze.)

Fl. Trop. Afr. iii. 322 ; Fl. Cap. iii. 119 ; Cat. Afr. Pl. Welw. i. 555.

Matopos, Sep. Gibbs, 269 ; Victoria Falls, Engler; Sep. Galpin, 7031 ; Bulawayo, Chubb, 302.

B. sp.

Victoria Falls, Rogers, 7421.

8940-Laggera alata, Sch. Bip.

Fl. Trop. Afr. iii. 326 ; Nat. Pl. 324 ; Cat. Afr. Pl. Welw. i. 556.

Matopos, Galpin, 6956; Rogers, 5179; Salisbury, April, Flanagan, 3053; Chirinda, 3800 ft. May, Swyn. 498; Odzani River Valley, Umtali, Teague, 133.

L. brevipes, Oliv. \& Hiern.

Fl. Trop. Afr. iii. 327 ; Cat. Afr. Pl. Welw. i. 557.

Chirinda, 3500 ft. July, Swyn. 2066.

L. pterodonta, Sch. Bip. (L. purpurascens, Sch. Bip.)

Fl. Trop. Afr. iii. 324.

Salisbury, July, Aug. Rand, 480, 500 ; Mazoe, 4800 ft. June, Eyles, 376 ; Matopos, Rogers, 5385.

8941-Pluchea Dioscoridis, DC.

Fl. Trop. Afr. iii. 329.

Victoria Falls, Rogers, 7134*.

8949-Denekia capensis, Thunb.

Fl. Trop. Afr. iii. 331 ; Fl. Cap. iii. 119 ; Nat. Pl. 365.

Crocodile Riv. Oates; Matopos, Galpin, 6961; Nov. Eyles, 1118; Gibbs, 39 ; Victoria Falls, May, Eyles, 138; Gibbs, 156 ; Allen, 405 ; Rogers, 5278, 5039 ; Victoria, Monro, 335 ; Gwelo, Jan. Gardner, 45 ; Marandellas, Engler ; Mazoe, $4200 \mathrm{ft}$. Sep. Eyles, 406.

8951-Nicolasia nitens, S. Moore.

Victoria Falls, Rogers, 7099*.

8953-Epaltes gariepina, Steetz.

Fl. Trop. Afr. iii. 332 ; Cat. Afr. Pl. Welw. i. 558.

Bulawayo, May, Rand, 354 ; Chubb, 360 ; Khami Riv. Oct. Eyles, 1085 ; Matopos, Oct. Gibbs, 195; Engler; Chipete Forest, 3800 ft. April, Swyn. 198; North Melsetter, Swyn. 1819. 
Genus No.

8954-Porphyrostemma alata?.

Umtali, Rogers, 4036.

8955 - Sphæranthus peduncularis, $D C$.

Fl. Cap. iii. 115.

Bulawayo, May, Rand, 355 ; Aug. Eyles, 1231.

S. Randii, S. Moore.

Journ. Bot. 1908, 40.

Salisbury, Rand, 527 ; Mazoe, 4200 ft. Sep. Eyles, 405.

S. Steetzii, Oliv. \& Hiern.

Fl. Trop. Afr. iii. 334.

Victoria Falls, Sep. Galpin, 7030.

\section{2 - Amphidoxa sp.}

Gwelo, Jan. Gardner, 51.

8990-Achyrocline batocana, Oliv. \& Hiern.

Fl. Trop. Afr. iii. 339.

Matopos, Rogers, 5190 ; Macheke, Rogers, 4046.

8992 - Gnaphalium luteo-album, $L$.

Fl. Trop. Afr. iii. 313 ; Fl. Cap. iii. 262.

Bulawayo, Dec. Rand, 136; Salisbury, Rand, 151 ; Bulawayo, 4600 ft. Aug. Eyles, 1236 ; Matopos, Sep. Gibbs, 40 ; Roger's, 5248 ; Gwelo, Jan. Gardner, 12.

9006_Helichrysum acervatum, S. Moore.

Journ. Linn. Soc. Bot. xl. 109.

Chimanimani Mts. 7000 ft. Sep. Swyn. 1845.

H. adenocarpum, $D C$.

Fl. Trop. Afr. iii. 350 ; Fl. Cap. iii. 229.

Chirinda, Chipetzana Riv., Nyahodi Riv. fl. April, May, Swyn. 1842, 1843, 1843a, $1843 b$.

H. appendiculatum, Less.

Fl. Cap. iii. 242.

Chirinda, 3700 ft. May, Swyn. 1851 ; Upper Buzi, 3500 ft. Nov. Swyn. 1851a.

H. argyrosphærum, $D C$.

Fl. Trop. Afr. iii. 351 ; Fl. Cap. iii. 222 ; Cat. Afr. Pl. Welw. i. 562. Bulawayo, Galpin, 7063 ; April, Flanagan, 3056 ; Matopos, Sep. Gibbs, 27 ; Engler; Chirinda, 3700 ft. Aug. Swyn. 1806.

H. auriculatum, Less.

Fl. Trop. Afr. iii. 347 ; Fl. Cap. iii. 253; Cat. Afr. Pl. Welw. i. 562 .

Chirinda, 3700 ft. June, Swyn. 1838.

H. brunioides, S. Moore.

Journ. Linn. Soc. Bot. xl. 111.

Chimanimani Mts. 7000 ft. Sep. Swyn. 1847. 
9006-H. Buchanani, Engl.

Melsetter, 6000 ft. Sep. Swyn. 1905.

H. cæspititium, Sond.

Fl. Cap. iii. 217.

Bulawayo, Jan. Rand, 100.

H. callicomum, Harv.

Fl. Cap. iii. 247.

Salisbury, May, Flanagan, 3048.

H. cymosum, Less.

Fl. Trop. Afr. iii. 353; Fl. Cap. iii. 245.

Matopos, 4500 ft. Feb. Eyles, 1169; Rogers, 5188; Umtali, Engler.

H. declinatum, Less.

Fl. Cap. iii. 218.

Salisbury, Dec. Rand, 152; Bulawayo, May, Rand, 351; Victoria Falls, Rogers, 5470.

H. ericæfolium, Less.

Fl. Cap. iii. 217.

Màtopos, Sep. Gibbs, 10.

H. fulgidum, Willd.

Fl. Cap. iii. 232.

Matopos, Rogers, 5653.

H. gazense, S. Moore.

Journ. Linn. Soc. Bot. xl. 110.

Melsetter, 6000 ft. Sep. Swyn. 1853.

H. Kraussii, Sch. Bip.

Fl. Cap. iii. 249 ; Nat. Pl. 269.

Salisbury, July, Rand, 531; Matopos, Engler; Melsetter, May, Swyn. 1835; Upper Buzi, 3600 ft. Sep. Swyn. $1835 a$.

H. latifolium, Less.

Fl. Cap. iii. 237.

Umtali, Rogers, 4030 ; Chirinda, May, Swyn. 510.

H. leiopodium, $D C$.

Fl. Cap. iii. 239 ; Cat. Afr. Pl. Welw. i. 565.

Victoria, Monro, 859; Chirinda, 3800 ft. April, Swyn. 307;

Lusitu Riv. 4000 ft. May, Swyn. 502 ; Mt. Pene, 65007000 ft. Sep. Swyn. 1837, 1852.

H. leptolepis, $D C$.

Fl. Cap. iii. 222.

Bulawayo, May, Rand, 348 ; Matopos, Sep. Gibbs, 11 ; Mazoe, April, Flanagan, 3055; Victoria, Monro, 452 ; Bulawayo, Aug. Eyles, 1246. 
H. nitens, Oliv. \& Hiern.

Fl. Trop. Afr. iii. 350.

Chirinda, Chimanimani Mts., Mt. Pene, Melsetter, in fl. Aug.Oct. Swyn. 280, 1836, 1839, 1840, 6111.

H. quinquenerve, Less.

Fl. Cap. iii. 240.

Victoria Falls, Rogers, 5688*.

H. Saweri, S. Moore.

Journ. Bot. 1905, 170.

Penhalonga, $6000 \mathrm{ft}$. E. R. Sawer.

H. setosum, Harv.

Fl. Cap. iii. 231.

Salisbury, July, Rand, 499 ; Bulawayo, 4500 ft. Feb. Eyles, 1170; Matabeleland, Marloth; Mazoe, 4300 ft. April, Eyles, 350 ; Matopos, Rogers, 5653 ?; Chirinda, Swyn. 279, 279a ; Melsetter, 6000 ft. April, Swyn. 281.

H. stenopterum, $D C$.

Fl. Cap. iii. 244.

Salisbury, April, Flanagan, 3044.

var. citrinum, S. Moore.

Journ. Bot. 1908, 41.

Mazoe, 4400 ft. April, Eyles, 363 ; Melsetter, June, Swyn. 1841 ; Chirinda Sep. Swyn. 1841a.

H. Swynnertonii, S. Moore.

Journ. Linn. Soc. Bot. xl. 109.

Melsetter, 6000 ft. Oct. Swyn. 6110.

H. spp.

Sicumy Vlei, $3200 \mathrm{ft}$. Allen, 230, cf. H. setosum, Harv.

Bulawayo, 4500 ft. June, Eyles, 152.

Matopos, Rogers, 5182, 5192.

9019-Humea africana, S. Moore.

Journ. Linn. Soc. Bot. xl, 112.

Chimanimani Mts. 7000 ft. Sep. Swyn. 1849.

9055-Athrixia elata, Sond.

Fl. Cap. iii. 292.

Mazoe, Sep. Rand, 484 ; July, Eyles, 383 ; O Jzani River Valley, Umtali, Teague, 196.

A. foliosa, S. Moore.

Journ. Linn. Soc. Bot. x]. 113.

Melsetter, 6000 ft. July, Swyn. 1820.

A. oblonga, S. Moore.

Journ. Linn. Soc. Bot. xl. 112.

Chirinda, 3800 ft. Oct. Swyn. 276. 
Genus No.

B. rosmarinifolia, Oliv. \& Hiern.

Fl. Trop. Afr. iii. 355.

North Melsetter, 5000-6000 ft. Umvumvumvu, $4000 \mathrm{ft}$. Oct. Swyn. 6135, 6137, 6137a, 6219 .

9061-Inula acervata, S. Moore.

Chirinda, 3800 ft. May, Swyn. 460.

I. glomerata, Oliv. \& Hiern.

Fl. Trop. Afr. iii. 359 ; Cat. Afr. Pl. Welw. i. 566.

Umtali, Engler, Odzani River Valley, Teague, 155.

9069-Calostephane divaricata, Benth.

Fl. Trop. Afr. iii. 363 ; Cat. Afr. Pl. Welw. i. 569.

North of Bulawayo, $3400 \mathrm{ft}$. May, Eyles, 82 ; between Salisbury and Bulawayo, April, Flanagan, 3069 (var.).

C. sp.

Salisbury, April, Flanagan, 3062.

9073 - Pegolettia senegalensis, Cass.

Fl. Trop. Afr. iii. 361; Cat. Afr. Pl. Welw. i. 568.

Deka, 2400 ft. May, Eyles, 131 ; Victoria Falls, April, Flanagan, 3071 ; Rogers, 5063, 7052.

9078-Pulicaria capensis, $D C$.

Fl. Cap. iii. 121.

Bulawayo, May, Rand, 356 ; Matopos, Rogers, 5243.

P. longifolia, Boiss.

Gwelo, Jan. Gardner, 48

9083-Philgrophyllum Schinzii, O. Hoffm.

Matopos, Rogers, 7928*; Wankie, Rogers*.

9087-Sphacophyllum flexuosum, Hutch.

Kew Bull. 1906, 249.

Chimanimani Mts. 7000 ft. Sep. Swyn. 1866.

S. sparsum, S. Moore.

Journ. Linn. Soc. Bot. xl. 114.

Lusitu Riv. 3000 ft. April, Swyn. 1867.

9090-Geigeria passerinoides, Harv.

Fl. Cap. iii. 125.

Matopos, 4600 ft. March, Eyles, 1035.

G. protensa, Harv. var. pubigera, S. Moore.

Journ. Bot. 1899, 375 ; Fl. Cap. iii. 125 (type).

Bulawayo, Dec. Rand, 104.

G. pubescens, S. Moore.

Journ. Bot. 1899, 374.

Bulawayo, May, Rand, 345. 
G. Randii, S. Moore.

Journ. Bot. 1899, 374.

Bulawayo, May, Rand, 346.

G. rhodesiana, S. Moore.

Journ. Bot. 1908, 41.

Mazoe, 4300 ft. April, Eyles, 318; Flanagan, 3061; Victoria, Monro, 968; Chirinda, 3800 ft. 490, Nyahodi Riv. 5000 ft., Chipetzana Riv. 3000 ft. fl. April, May, Swyn. 490, 1817, 1823.

G. Zeyheri, Harv.

Fl. Cap. iii. 126.

Crocodile Riv. Oates; Matopos, April, Flanagan, 3059.

G. spp.

Matopos, April, Flanagan, 3060, ef. G. africana, Gr.

Matopos, Eyles, 1059.

Bulawayo, Chubb, 323.

9148-Xanthium spinosum, $L$.

Bulawayo, 4500 ft. Eyles, 99 ?; Salisbury, Govt. Herb. 797.

9160_Siegesbeckia abyssinica, Oliv. \& Hiern.

Fl. Trop. Afr. iii. 372.

Victoria Falls, Rogers, 5032.

9169-Sclerocarpus africanus, Jacq.

Fl. Trop. Afr. iii. 374 ; Cat. Afr. Pl. Welw. i. 575.

Salisbury, April, Flanagan, 3037.

9192-Wedelia abyssinica, Vatke.

Fl. Trop. Afr. iii. 377.

Umtali, Rogers, 4063*; Bulawayo, 13559*.

W. africana, P. Beauv.

Fl. Trop. Afr. iii. 376 ; Cat. Afr. Pl. Welw. i. 576.

Salisbury, April, Flanagan, 3037a.

W. diversipapposa, S. Moore.

Journ. Bot. 1899, 401.

Bulawayo, Jan. Rand, 111.

W. menotriche, Oliv. \& Hiern.

Fl. Trop. Afr. iii. 377.

Bulawayo, Rogers, 5910*; Salisbury, Rogers, 13089*.

\section{W. spp.}

Victoria Falls, Allen, 10, apparently undescribed.

Victoria Falls, Allen, 21, cf. W. Menotriche, Oliv. \& Hiern.

Bulawayo, Chubb, 333.

9195-Aspilia brachyphylla, S. Moore.

Journ. Linn. Soc. Bot. xl. 115.

Chirinda, May, Swyn. 292, 495 ; Salisbury, Rogers, 13092*. 
9411-ї. Eylesii, S. Moore.

Journ. Bot. 1907, 45.

Sebakwe, 4000 ft. Dec. Eyles, 164.

A. vulgaris, N. E. Br.

Kew Bull. 1906, 164.

Between Salisbury and Umtali, Cecil, 43.

A. zombensis, Baker.

Victoria Falls, Allen, 199.

A. sp.

Victoria Falls, Allen, 254, cf. A. Kotschyi, Bth. \& Hook.

9204-Melanthera Brownei, Sch. Bip.

Fl. Trop. Afr. iii. 382 ; Cat. Afr. Pl. Welw. i. 579.

Victoria Falls, Rogers, 5012, 5581; Chirinda, 3500 ft. Oct. Swyn. 293, 294.

9222-Guizotia abyssinica, Cass.

Fl. Trop. Afr. iii. 384.

Salisbury, April, Flanagan, 3039.

G. Eylesii, S. Moore.

Journ. Bot. 1908, 43.

Mazoe, 4300 ft. April, Eyles, 349 ; Chirinda, 3800 ft. May, Swyn. 295; Odzani River Valley, Umtali, Teague, 175.

9227-Coreopsis insecta, S. Moore.

Journ. Bot. 1908, 42.

Mazoe, $4700 \mathrm{ft}$. March, Eyles, 266.

C. Steppia, Steetz.

Fl. Trop. Afr. iii. 388; Cat. Afr. Pl. Welw. i. 584.

Mazoe, 4400 ft. April, Eyles, 307.

C. sp.

Between Wankie and Victoria Falls, Rogers, 6001.

9232-Chrysanthellum procumbens, Pers.

Fl. Trop. Afr. iii. 395 ; Cat. Afr. Pl. Welw. i. 588.

Bulawayo, Jan. Rand, 148; Jan. Gardner, 81; Rogers, 5926; Victoria Falls, Rogers, 6026*.

9237-Bidens pilosa, $L$.

Fl. Trop. Afr. iii. 392 ; Fl. Cap. iii. 133 ; Cat. Afr. Pl. Welw. i. 587.

Bulawayo, May, Rand, 340; Mazoe, 4300-4800 ft. March, Eyles, 296; Victoria Falls, Rogers; Odzani River Valley, Umtali, Teague, 149.

B. prolixus, S. Moore.

Journ. Linn. Soc. Bot. xl. 116.

Melsetter, fl. \& fr. April, Swyn, 1884. 
Genus No.

B. Schimperi, Sch. Bip.

Fl. Trop. Afr. iii. 393.

Victoria Falls, April, Flanagan, 3036, 3041; Gwelo, Kolbe.

9262-Jaumea compositarum, Benth. \& Hook.

Fl. Trop. Afr. iii. 395.

Salisbury, April, Flanagan, 3077 ; Gwelo, Sr. Phil. 36.

J. elata (N. E. Br.), Torre \& Harms. (Hypericophyllum elatum, N. E. Br.)

Chipetzana Riv. 3000 ft. April, Swyn. 1816.

9291-Schkuhria bonariensis, $L$.

Bulawayo, Rogers, 5911, a weed of cultivation.

9311-Tagetes glandulifera, Schrank.

Bulawayo, Monro, 79 ; Chubb, 341.

T. minuta, $L$.

Salisbury, March, Flanagan, 3015; Gwelo, Sr. Phil. 54; Bulawayo, Chubb, $10 a$.

9351-Cotula anthemoides, $L$.

Fl. Trop. Afr. iii. 397 ; Fl. Cap. iii. 182 ; Cat. Afr. Pl. Welw. i. 590.

Bulawayo, 4600 ft. Aug. Eyles, 1238; Monro, 36; Matopos, Rogers, 5245.

9356-Schistostephium artemisiifolium, Baker.

Umtali, Engler.

S. heptalobum, Benth. \& Hook.

Fl. Trop. Afr. iii. 399.

Salisbury, April, Flanagan, 3041 ; Chirinda, 3500 ft. May, Swyn. 491 ; Melsetter, 6000 ft. Sep. Swyn. 1808.

S. oxylobum, S. Moore.

Journ. Linn. Soc. Bot. xl. 117.

Chimanimani Mts. 7000 ft. Sep. Swyn. 1871; Mt. Pene, 7000 ft. Oct. Swyn. 6132.

9358-Artemisia afra, Jacq.

Fl. Cap. iii. 170; Cat. Afr. Pl. Welw. i. 590 ; Harv. Gen. S.A. Pl. 185.

Crocodile Riv. Oates; Salisbury, July, Rand, 496; April, Flanagan, 3045 ; Mazoe, 4300 ft. April, Eyles, 351 ; Chirinda, 3700 ft. July, Swyn. 446; Melsetter, 6000 ft. Swyn. 646'; Lusitu Riv. Swyn. 646a, 646b; Nyahodi Riv. Swyn. 1822; Odzani River Valley, Umtali, Teague, 92.

9374-Congrothamus sp.

Victoria, Monro 1018. 
A Record of Plants Collected in Southern Rhodesia.

Genus No.

9401-Ĺopholæna brickellioides, S. Moore.

Journ. Linn. Soc. Bot. xl. 118.

Chimanimani Mts. 7000 ft. Sep. Swyn. 1802 ; Mt. Pene, 7000 ft.

Oct. Swyn. 6033.

L. Randii, S. Moore.

Gwelo, Sr. Phil. 4.

9405-Gynura cernua, Benth. (Crassocephalum cernum, Moench.)

Fl. Trop. Afr. iii. 402 ; Cat. Afr. Pl. Welw. i. 593.

Salisbury, Aug. Rand, 493.

G. crepidoides, Benth. (Crassocephalum diversifolium, Hiern, var. crepidioides, Hiern.)

Fl. Trop. Afr. iii. 403 ; Cat. Afr. Pl. Welw. i. 594.

Salisbury, April, Flanagan, 3072.

G. sarcobasis, $D C$.

Matopos, $4800 \mathrm{ft}$. April, Eyles, 40.

G. vitellina, Benth .

Fl. Trop. Afr. iii. 402.

Mazoe, $4300 \mathrm{ft}$. Dec. Eyles, 227.

9406-Cineraria lobata, L'Herit.

Fl. Cap. iii. 311.

Melsetter, $6000 \mathrm{ft}$. Sep. Swyn. 1875.

C. mazoensis, S. Moore.

Journ. Bot. 1908, 43.

Mazoe, 5000--5200 ft. April, Eyles, 345.

9411-Senecio acervatus, S. Moore.

Journ. Linn. Soc. Bot. xl. 121.

Chirinda Forest, 3700-4000 ft. Oct. Swyn. 665.

S. barbertonicus, Klatt, var. microcephala, S. Moore.

Journ. Linn. Soc. Bot. xxxvii. 451.

Matopos, Oct. Gibbs, 250 ; Bulawayo, 4500 ft. Aug. Eyles, 1239.

S. bupleuroides, $D C$.

Fl. Trop. Afr. iii. 414 ; Fl. Cap. iii. 378.

Salisbury, Darling; May, Flanagan, 3079.

S. deltoideus, Less.

Fl. Trop. Afr. iii. 420 ; Fl. Cap. iii. 403 ; Nat. Pl. 382.

Chirinda, 3700-4000 ft. Feb. Swyn. 288; Chipete Forest, 3800

ft. June, Swyn. 288a; Mt. Pene, Oct. Swyn. 6131.

S. discifolius, Oliv.

Fl. Trop. Afr. iii. 410.

Gwelo, Jan. Gardner, 35. 
Genus No.

9411-S. erubescens, Ait.

Fl. Cap. iii. 363 ; Cat. Afr. Pl. Welw. i. 597.

Matopos, Oct. Gibbs, 241; Chirinda, 3700-4000 ft. Nov. Swyn.

1812 ; Macheke, Rogers, 4045*; Bulawayo, Rogers, 5519*.

S. gazensis, S. Moore.

Journ. Linn. Soc. Bot. xl. 121.

Melsetter, 6000 ft. Oct. Swyn. 6143.

S. homoplasticus, S. Moore.

Journ. Linn. Soc. Bot. xl. 120.

Chirinda, 3500 ft. Oct. Swyn. 288.

var. tomentellus, S. Moore.

Journ. Linn. Soc. Bot. xl. 121.

Chirinda, $3500 \mathrm{ft}$. Swyn. $288 a$.

S. lasiorhizus, DC. (S. coronatus, Harv.)

Fl. Trop. Afr. iii. 415; Fl. Cap. iii. 369 ; Cat. Afr. Pl. Welw.i. 598.

Bulawayo, Dec. Rand, 139 ; Nov. Eyles, 1222 ; Salisbury, Sep. Rand, 628 (var.); Engler; Darling; Matopos, Sep. Gibbs, 189 ; Salisbury, Rogers, 4026.

S. latifolius, $D C$.

Fl. Cap. iii. 377 ; Cat. Afr. Pl. Welw. i. 598.

Bulawayo, Dec. Rand, 140; Salisbury, Engler; Chirinda, Nov. Swyn. 1876, 1944; also Melsetter Dist. Swyn.

S. longiflorus, Oliv. \& Hiern. (Kleinia longiflora, DC.)

Bulawayo, 4500 ft. fl. Sep. fr. Oct. Eyles, 1083 ; Engler.

S. othonnæflorus, $D C$.

Fl. Cap. iii. 373 .

Bulawayo, 4500 ft. Nov. Eyles, 1223.

S. oxyriæfolius, $D C$.

Fl. Cap. iii. 376.

Chimanimani Mts. 7000 ft. Sep. Swyn. 1946; Bulawayo, Rogers, $13563 \%$.

S. picridifolius, $D C$.

Fl. Trop. Afr. iii. 413; Fl. Cap. iii. 379; Cat. Afr. Pl. Welw. i. 597.

Salisbury, April, Flanagan, 3076 ; Umtali, Rogers, 4052.

S. propior, S. Moore.

Journ. Linn. Soc. Bot. xl. 118.

Chimanimani Mts. 7000 ft. Sep. Swyn. 1879.

S. protracta (S. Moore), Torre \& Harms. (Emilia protracta, S. Moore.)

Journ. Bot. 1905, 48.

Victoria Falls, 3000 ft. May, Eyles, 119; April, Flanagan, 3234 ; Rogers, 5023. 
Genus No.

S. purpureus, $L$.

Fl. Cap. iii. 363.

Macheke, Rogers, 4048.

S. Randii, S. Moore.

Journ. Bot. 1899, 402.

Salisbury, Sep. Rand, 625.

S. rosmarinifolius, $L$. $f$.

Fl. Cap. iii. 100.

Matopos, Oct. Gibbs, 43 ; April, Flanagan, 3081; Gwelo, Sr. Phil. 31.

S. sagittata (DC.), Torre \& Harms. (Emilia sagittata, DC.; Emilia flammea, Cass.)

Fl. Trop. Afr. iii. 405; Cat. Afr. Pl. Welw. i. 595.

Mazoe, 5000 ft. March, Eyles, 288; April, Flanagan, 2057 ;

Odzani River Valley, Umtali, Teague, 16.

S. sarmentosus, $O$. Hoffm.

Mazoe, $4300 \mathrm{ft.}$ July, Eyles, 379.

S. Serra, Sond.

Fl. Cap. iii. 389.

Matopos, May, Flanagan, 3080.

S. tamoides, $D C$.

Fl. Cap. iii. 404 ; Nat. Pl. 95.

Chipete Forest, 3800 ft. Swyn. 1877.

S. tenellulus, S. Moore.

Journ. Linn. Soc. Bot. xxxvii. 449.

Matopos, Oct. Gibbs, 203.

\section{S. spp.}

Salisbury, April, Flanagan, 3073, cf. S. myriocephalus, Sch. Bip.

Salisbury, Darling, in Herb. Bolus, 10780.

Matopos, Rogers, 5183.

Victoria Falls, Allen, 15, cf. S. sagittata (DC.)

9417-Euryops osteospermum, S. Moore.

Journ. Bot. 1899, 403.

Salisbury, July, Rand, 498.

var. parvifolia, S. Moore.

Journ. Bot. 1899, 403.

Salisbury, Dec. Rand, 109.

9420-Othonna ambifaria, S. Moore.

Journ. Bot. 1899, 403.

Shashi Riv. Jan. Rand, 110.

9427-0steospermum herbaceum, Linn. $f$.

Fl. Cap. iii. 435.

Salisbury, Aug. Rand, 489. 
Genus No. moniliferum, Linn.

Fl. Cap. iii. 436 ; Nat. Pl. 55.

Salisbury, Aug. Rand, 487 (var.) ; Melsetter, 6000 ft. Swyn. 497 ; Chirinda, 3500 ft. Swyn, 1858; Mt. Pene, Swyn. 1858a; Haroni Riv. 5000 ft. Swyn. 6130 ; in fl. May, Sep. Oct. Swyn.

0. muricatum, E. Mey.

Fl. Trop. Afr. iii. 425 ; Fl. Cap. iii. 441 ; Cat. Afr. Pl. Welw. i. 607.

Salisbury, Aug. Rand, 339 ; Matopos, fl. \& fr. Sep. Gibbs, 22 ; Galpin, 6990 ; Bulawayo, Chubb, 351, 371; Rogers, 5932.

9428-Tripteris amplexicaulis, Less.

Fl. Cap. iii. 427 ; Cat. Afr. Pl. Welw. i. 607.

Salisbury, Sep. Rand, 485; Mazoe, 4300-4800 ft. Oct. Eyles, 437 ; Victoria, Monro, 727; Mt. Pene, 6500-7000 ft. Sep. Swyn. 1859.

T. monocephala, Oliv. \& Hiern.

Fl. Trop. Afr. iii. 424.

Salisbury, Rogers, 4001 ; Mt. Pene, 7000 ft. Oct. Swyn. 6161.

T. sp.

Salisbury, Darling, in Herb. Bolus, 10777, cf. T. monocephala, Oliv. \& Hiern.

9432-Arctotis scaposa (Harv.) Torre \& Harms. (Haplocarpha scaposa, Harv.)

Fl. Trop. Afr. iii. 427 ; Fl. Cap. iii. 465.

Bulawayo, Dec. Rand, 99; Gwelo, Jan. Gardner, 80 ; Plumtree, April, Flanagan, 3032.

9434-Gazania krebsiana, Less.

Fl. Cap. iii. 475.

Salisbury, Rand, 490.

var. hispidula, Harv.

Fl. Cap. iii. 476.

Mazoe, 4300 ft. Aug. Eyles, 184; Matopos, fl. \& fr. Sep. Gibbs, 75 ; Victoria, Monro, 444 ; Mt. Pene, Oct. Swyn. 6115.

9436-Berkheyopsis integrifolia, Volk.

Bulawayo, Dec. Rand, 133.

B. bechuanensis, S. Moore.

Bulawayo, Rogers, 5496*, 13554*.

B. spp.

Plumtree, April, Flanagan, 3033.

9438-Berkheya Adlami, Hook. $f$.

Sebakwe, 4000 ft. Dec. Eyles, 118; Victoria, Monro, 964 ; Bulawayo, Rand, 149. 
A Record of Plants Collected in Southern Rhodesia.

Genus No

B. gorterioides, Oliv. \& Hiern.

Fl. Trop. Afr. iii. 429.

Bulawayo, Rogers, 13555*.

B. setifera, $D C$. var. tropica, S. Moore.

Journ. Linn. Soc. Bot. xl. 123 ; Fl. Cap. iii. 509 (type).

Lusitu Riv. 5000 ft. Sep. Swyn. 1869.

B. subulata, Harv.

Fl. Cap. iii. 507 ; Nat. Pl. 225.

Mazoe, 4500 ft. Aug. Eyles, 178; Salisbury, Darling; Odzani River Valley, Umtali, Teague, 9.

B. Zeyheri, Sond. \& Harv.

Fl. Trop. Afr. iii. 429.

Gwelo, Jan. Gardner, 6; Bulawayo, Jan. Rand, 113 ; Haroni Riv. 5600 ft. Sep. Swyn. 1868; Melsetter, 6000 ft. Oct. Swyn. 6114.

B. spp.

Salisbury, Rogers, 4065.

Victoria Falls, Flanagan, 3031, 3034.

9476-Centaurea rhizocephala, Oliv. \& Hiern. var. australis?.

Fl. Trop. Afr. iii. 438 (type).

Salisbury, Aug. Rand, 482 ; Mazoe, $4400-4800$ ft. Aug. Eyles, 397.

C. sp.

Salisbury, Darling in Herb. Bolus, 10776.

9499-Pleiotaxis Antunessii, O. Hoffm.

Cat. Afr. Pl. Welw. i. 611.

Mazoe, 4500-5000 ft. March, Eyles, 287 ; April, Flanagan, 3054.

P. spp.

Gwelo, Sr. Phil. 2 ; Salisbury, Rogers, 4079.

9501-Dicoma anomala, Sond.

Fl. Trop. Afr. iii. 443 ; Fl. Cap. iii. 517 ; Cat. Afr. Pl. Welw. i. 614.

Bulawayo, June, Rand, 486; Salisbury, Darling; Matopos, Rogers, 5358 ; Victoria Falls, Rogers, 7151 ; North Melsetter, April, Swyn. 1818; Odzani River Valley, Umtali, Teague, 153.

var. microcephala, Harv.

Fl. Trop. Afr. iii. 443 ; Fl. Cap. iii. 517.

Matopos, 4500 ft. Feb. Eyles, 1176 ; Victoria, Monro, 883.

var. Sonderi, Harv.

Fl. Trop. Afr. iii. 443 ; Fl. Cap. iii. 517.

Plumtree, April, Flanagan, 3029.

D. Kirkii, Harv.

Fl. Trop. Afr. iii. 444 ; Fl. Cap. iii. 518.

Salisbury, May, Flanagan, 3051; Gwelo, Sr. Phil. 41; Odzani River Valley, Umtali, Teague, 154. 
Genus No.

var. angustifolia, S. Moore.

Journ. Linn. Soc. Bot. xl. 123.

Chirinda, 3800 ft. May, Swyn. 444 ; Victoria, Monro, 413, 1100c.

D. Schinzii, O. Hoffm.

Victoria Falls, Rogers, 5300 (var.).

D. sessiflora, Harv.

Fl. Trop. Afr. iii. 444 ; Fl. Cap. iii. 518.

Mazoe, 4800 ft. Aug. Eyles, 186 (var. ?).

D. tomentosa, Cass.

Fl. Trop. Afr. iii. 443 ; Cat. Afr. Pl. Welw. i. 613.

Deka, 2400 ft. May, Eyles, 136.

9504-Erythrocephalum zambesianum, Oliv. \& Hiern.

Fl. Trop. Afr. iii. 441.

Chirinda, Oct. Swyn. 236.

9528-Gerbera abyssinica, Sch. Bip. (Perdicium abyssinicum, Hiern.) Fl. Trop. Afr. iii. 445 ; Cat. Afr. Pl. Welw. i. 615.

Salisbury, Sep. Rand, 623 ; Gwelo, Jan. Gardner, 36 ; Mt. Pene, 6500 ft. Sep. Oct. Swyn. 1821, 6113.

G. ambigua, Sch. Bip.

Fl. Cap. iii. 522.

Umtali, Rogers, 4033.

G. discolor, Sond.

Fl. Cap. iii. 522.

Salisbury, April, Flanagan, 3043.

G. piloselloides, Cass. (Perdicium piloselloides, Hiern.)

Fl. Trop. Afr. iii. 445 ; Fl. Cap. iii. 522 ; Cat. Afr. Pl. Welw. i. 615.

Salisbury, Dec. Rand, 112 ; Matopos, fl. \& fr. Oct. Gibbs, 257 ;

Chirinda, $4500 \mathrm{ft.} \mathrm{Nov.} \mathrm{Swyn.} 1870$.

G. plantaginea, Harv.

Fl. Cap. iii. 522.

Mazoe, 4800 ft. Aug. Eyles, 400 ?

G. viridifolia, Sch. Bip. (G. natalensis, Sch. Bip.)

Fl. Cap. iii. 523 ; Nat. Pl. 546.

Crocodile Riv. Oates; Salisbury, Darling; Rogers, 4006, 4005.

G. sp.

Salisbury, Rogers, 4076.

9561-Tolpis sp. (Swertia sp.)

Victoria, Monro, 580.

9595-Sonchus Bipontini, Aschers. var. pinnatifidus, Oliv. \& Hiern.

Fl. Trop. Afr. iii. 458.

Chirinda, 3800 ft. May, Swyn. 1886. 
Genus No.

S. elliotanus, Hiern.

Cat. Afr. Pl. Welw. i. 623.

Salisbury, Sep. Rand, 483 ; Bulawayo, Chubb, 348.

S. macer, S. Moore.

Journ. Bot. 1899, 404.

Salisbury, Sep. Rand, 627.

S. oleraceus, $L$.

Fl. Trop. Afr. iii. 457 ; Fl. Cap. iii. 528 ; Cat. Afr. Pl. Welw. i. 622. Gwelo, Sr. Phil. 45 ; a common weed, Rogers.

S. violaceus, O. Hoffm .

Salisbury, Rogers, 4062*.

9596-Lactuca capensis, Thunb.

Fl. Trop. Afr. iii. 452 ; Fl. Cap. iii. 526 ; Cat. Afr. Pl. Welw. i. 621.

Bulawayo, May, Rand, 349 ; Jan. Eyles, 67 ; Chubb, 374, 376, 381 ; Salisbury, July, Rand, 488; Darling; Matopos, Nov Eyles, 1149 ; fl. \& fr. Sep. Gibbs, 52 ; Gwelo, Jan. Gardner, 39 ; North of Hartley, Engler; Mazoe, 4300-4800 ft. Oct. Eyles, 438; Victoria, Monro, 446, 446a; Odzani River Valley, Umtali, Teague, 76, 122, 123.

L. virosa, Thunb. (L. dregeana, DC.)

Fl. Cap. iii. 526.

Matopos, Engler. 


\section{$2 \mathrm{BHL}$ Biodiversity Heritage Library}

Eyles, Fred. 1915. "A RECORD OF PLANTS COLLECTED IN SOUTHERN

RHODESIA." Transactions of the Royal Society of South Africa 5, 273-523.

https://doi.org/10.1080/00359191509519725.

View This Item Online: https://www.biodiversitylibrary.org/item/181589

DOI: https://doi.org/10.1080/00359191509519725

Permalink: https://www.biodiversitylibrary.org/partpdf/175551

\section{Holding Institution}

Smithsonian Libraries

\section{Sponsored by}

Biodiversity Heritage Library

\section{Copyright \& Reuse}

Copyright Status: Not in copyright. The BHL knows of no copyright restrictions on this item.

This document was created from content at the Biodiversity Heritage Library, the world's largest open access digital library for biodiversity literature and archives. Visit BHL at https://www.biodiversitylibrary.org. 